\title{
One-step RESOLFT with a positively switchable RSFP with improved deactivation kinetics
}

\author{
Dissertation \\ to acquire the doctoral degree in mathematics and natural science \\ 'Doctor rerum naturalium' \\ at the Georg-August-Universität Göttingen \\ in the doctoral degree programme biology \\ at the Georg-August University School of Science (GAUSS)
}

submitted by

Timo Konen

from Papenburg

Göttingen, 2019 

Thesis committee:

Prof. Dr. Stefan Jakobs

Department of NanoBiophotonics

Max Planck Institute for Biophysical Chemistry

Prof. Dr. Kai Tittmann

Molecular Enzymology

Georg-August-University Göttingen

Members of the examination board:

Referee:

Prof. Dr. Stefan Jakobs

Department of NanoBiophotonics

Max Planck Institute for Biophysical Chemistry

Co-referee:

Prof. Dr. Kai Tittmann

Molecular Enzymology

Georg-August-University Göttingen

Other members of the examination board:

apl. Prof. Dr. Alexander Egner

Optical Nanoscopy

Laser Laboratory Göttingen

Prof. Dr. Dr. h.c. mult. Stefan W. Hell

Department of NanoBiophotonics

Max Planck Institute for Biophysical Chemistry

PD Dr. Wilfried Kramer

Department of Molecular Genetics

Institute for Microbiology and Genetics

PD Dr. Thomas Teichmann

Department of Plant Cell Biology

Georg-August-University Göttingen

Date of the oral examination: December $11^{\text {th }}, 2019$ 



\section{Abstract}

Interaction and spatial organization of subcellular structures within living cells are an essential aspect of biological research, but conventional microscopy can elucidate these properties only to a finite extent as it is limited in its optical resolution by the diffraction barrier. In recent years, this boundary has continuously been pushed and ultimately been broken in the field of nanoscopy. Reversible saturable optical linear fluorescence transition (RESOLFT) super-resolution microscopy as one of these methods is particularly suited for nanoscopy of live cells. It utilizes the transition of fluorophores between a fluorescent on-state and a nonfluorescent off-state and breaks the diffraction barrier at low light intensities in the range of $10^{3} \mathrm{~W} / \mathrm{cm}^{2}$. Reversibly switchable fluorescent proteins (RSFPs) are established probes for RESOLFT nanoscopy. Most implementations rely on negatively switchable RSFPs, which transition to the fluorescent off-state in a process driven by the same wavelength that facilitates fluorescence excitation and that can be reversed with light of another wavelength. Fluorescence emission and transition to the off-state are competing processes that set a limited photon budget for RESOLFT nanoscopy. This can be circumvented by positively switchable RSFPs that have a reversed light response: their transition to the on-state is driven by their fluorescence excitation wavelength, which can be subdued by another wavelength that facilitates the inverse transition. So far, the poor performance of available positively switchable RSFPs such as Padron, asFP595, and rsCherry has limited their use for nanoscopy and only few demonstrations have been published. This thesis reports the generation of Padron 2.0, which was created by multiple rounds of mutagenesis and screening from Padron.

Padron 2.0 is a significant improvement over Padron in key aspects of fluorescence state transition and is therefore particularly suited for RESOLFT nanoscopy. In contrast to Padron, Padron 2.0 displayed good expression and maturation at $37^{\circ} \mathrm{C}$ as well as enhanced capabilities as a protein tag in live-cell applications. Most importantly, key aspects relevant for RESOLFT nanoscopy were improved. Residual ensemble off-state fluorescence after switching was below $1 \%$, and transition to this state was more than one order of magnitude faster compared to Padron. The number of switching cycles before an ensemble of Padron 2.0 fluorophores was bleached to $50 \%$ fluorescence intensity was increased more than 50-fold. Padron 1.9 and Padron 2.1 are presented as direct precursor and successor of Padron 2.0. They showed sim- 
ilar but less pronounced improvements and were characterized as candidates for potential niche applications. While Padron 1.9 is brighter than Padron 2.0 but more prone to switching fatigue, padron 2.1 displays more efficient transition to the off-state but is darker and tends towards oligomerization.

RESOLFT nanoscopy of Padron 2.0-labeled vimentin structures in live HeLa cells featured a lateral resolution of single filaments as low as $60 \mathrm{~nm}$. In addition, the unique switching characteristics of Padron 2.0 allowed for the establishment of a novel RESOLFT imaging mode that was termed "one-step RESOLFT" in this thesis. As off-switching was more dominant than on-switching in Padron 2.0, a simplified imaging sequence with a steady-state approach and short pixel dwell times of $300 \mu \mathrm{s}$ was demonstrated that achieved similar lateral resolution as classical RESOLFT schemes but reduced bleaching during time-lapse imaging.

Padron 2.0 was shown to be the most robust and versatile positively switching RSFP to date and constitutes a promising candidate for further applications. 


\section{Table of contents}

1 Introduction 1

1.1 Microscopy . . . . . . . . . . . . . . . . . . . . . . . 1

1.1.1 Fluorescence microscopy . . . . . . . . . . . . . . . . . . . . 1

1.1.2 Diffraction barrier in light microscopy . . . . . . . . . . . . . . 3

1.1.3 Diffraction-unlimited microscopy . . . . . . . . . . . . . . 5

1.1.3.1 Coordinate-stochastic nanoscopy . . . . . . . . . . 5

1.1.3.2 Coordinate-targeted nanoscopy . . . . . . . . . . . . 7

1.2 Fluorescent proteins $\ldots \ldots \ldots \ldots \ldots \ldots$

1.2.1 Chromophore maturation and spectral properties of GFP . . . . . 10

1.2.2 Spectral variation of GFP-like proteins . . . . . . . . . . . . . . . 12

1.2.3 Reversibly switchable fluorescent proteins . . . . . . . . . . . . . . 14

1.2.3.1 Switching mechanism of RSFPs . . . . . . . . . . 15

1.2.3.2 Switching characteristics . . . . . . . . . . . 16

1.2.4 RSFPs in RESOLFT nanoscopy . . . . . . . . . . . . . . . 18

1.2.5 Nanoscopy with positively switchable RSFPs . . . . . . . . . . . 20

2 Aim of thesis 23

3 Results $\quad 24$

3.1 Improvement of the automated screening microscope . . . . . . . . . . . . . 24

3.1.1 Improving speed and precision of colony sampling . . . . . . . . . . . 24

3.1.2 Accelerating the autofocus . . . . . . . . . . . . . 26

3.2 Padron mutagenesis . . . . . . . . . . . . . . . . . . 27

3.3 Switching parameters improvement . . . . . . . . . . . . . . . . 29

3.3.1 Switching characteristics of intermediate variants . . . . . . . . . . 31

3.3.2 Bright intermediate state during activation of intermediate variants . 34

3.4 Detailed characterization of Padron 1.9, 2.0, and 2.1 . . . . . . . . . . . 36

3.4.1 Characterization of switching characteristics . . . . . . . . . 36

3.4.1.1 Residual off-state fluorescence intensity . . . . . . . . . . . 36

3.4.1.2 On-Switching kinetics . . . . . . . . . . . . . . . . 38

3.4.1.3 Switching fatigue . . . . . . . . . . . . . . . . 41

3.4.1.4 Photobleaching . . . . . . . . . . . . . . . 43

3.4.1.5 Switching background with simultaneous illumination . . . . 44

3.4 .2 In vitro switching and metastability . . . . . . . . . . . . . . . . 46

3.4.3 Spectral properties . . . . . . . . . . . . . . . . 48

3.4.3.1 Absorption and emission spectra of switching states . . . . 48

3.4.3.2 $\mathrm{pH}$ dependent absorption spectra . . . . . . . . . . 51

3.4.3.3 Extinction coefficient, quantum yield and fluorescence lifetime 53 
3.4 .4 Tagging and oligomerization . . . . . . . . . . . . . . 54

3.4.4.1 In vitro oligomerization tendencies . . . . . . . . . . . 55

3.4.4.2 Size exclusion chromatography . . . . . . . . . . . 56

3.4.4.3 Labeling of cellular structures with Padron variants . . . . . 57

3.5 Super-resolution microscopy with Padron $2.0 \ldots \ldots$. . . . . . . . . 61

3.5.1 Evaluation of vimentin-Padron 2.0 structures with STED nanoscopy . 61

3.5.2 RESOLFT microscopy . . . . . . . . . . . . . . . . 63

3.5.3 Comparison of sequential and one-step RESOLFT imaging . . . . . . 69

4 Discussion $\quad 72$

4.1 Improvement of the automated screening microscope . . . . . . . . . . . . 72

4.2 Generation of a new Padron variant . . . . . . . . . . . . . . . 73

4.2 .1 Padron . . . . . . . . . . . . . . . . . . 73

4.2 .2 Kohinoor . . . . . . . . . . . . . . . . . 75

4.2.3 Generation of the new Padron variants . . . . . . . . . . . 76

4.2.3.1 Influence of arginine 66, alanine 69 , and leucine $141 \ldots 78$

4.2.3.2 Influence of other positions . . . . . . . . . . . 81

4.2.4 The bright intermediate state . . . . . . . . . . . . . . 84

4.2.5 Improved protein and switching characteristics of the new Padron variants 86

4.2.5.1 Padron 1.9 and 2.1 . . . . . . . . . . . . . . 90

4.2 .6 RESOLFT nanoscopy with Padron $2.0 \ldots \ldots$. . . . . . . . 90

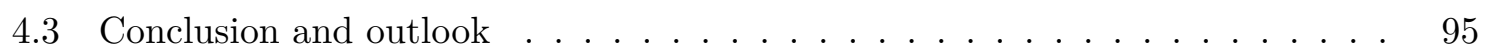

5 Methods $\quad 97$

5.1 Protein expression . . . . . . . . . . . . . . . . . . . . 97

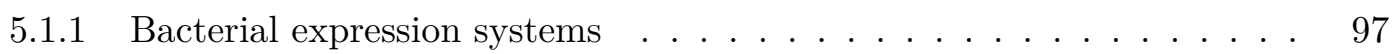

5.1.1.1 Plasmids . . . . . . . . . . . . . . . . 97

5.1.1.2 Bacterial strains . . . . . . . . . . . . . . . 97

5.1.1.3 Transformation . . . . . . . . . . . . . . 98

5.1.1.4 Growth conditions . . . . . . . . . . . . . . 98

5.1 .2 Mammalian expression systems . . . . . . . . . . . . . . . . . 99

5.1.2.1 Mammalian plasmids . . . . . . . . . . . . . . 99

5.1.2.2 Cell line and growth conditions . . . . . . . . . . . . . 102

5.1 .2 .3 Transfection . . . . . . . . . . . . . . . . 102

5.2 Mutagenesis . . . . . . . . . . . . . . . . . . 103

5.2 .1 Cloning protocols . . . . . . . . . . . . . . . . . . 103

5.2 .2 Site-directed mutagenesis . . . . . . . . . . . . . . . . . . 104

5.2 .3 Multiple-site mutagenesis . . . . . . . . . . . . . . . . . . . . . 104

5.2 .4 Error-prone mutagenesis . . . . . . . . . . . . . . . . . . . 105

5.2.5 Plasmid isolation from bacterial cultures . . . . . . . . . . . . . 105

5.2 .6 DNA sequencing . . . . . . . . . . . . . . . . 106 


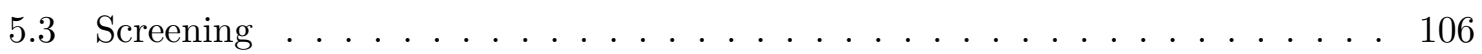

5.3 .1 Automated bacterial screening . . . . . . . . . . . . 106

5.3.1.1 Technical specifications of the screening setup . . . . . . 106

5.3.1.2 Improved autofocus evaluation . . . . . . . . . . . . . . 108

$5.3 .1 .3 \quad$ Screening strategy . . . . . . . . . . . . . . . 108

5.3.1.4 Fluorescent protein characterization in bacterial colonies . . 109

5.4 Protein purification . . . . . . . . . . . . . . . . . . 112

5.4 .1 Protein expression . . . . . . . . . . . . . . . . . 113

5.4 .2 Cell lysis . . . . . . . . . . . . . . . . . . . . . 113

5.4 .3 Ni NTA spin column protein isolation . . . . . . . . . . . . . . 113

5.4.4 Protein concentration and purity . . . . . . . . . . . . . . 114

5.4.5 Polyacrylamide gel electrophoresis . . . . . . . . . . . . . . . . . . 114

5.4.5.1 Denaturing PAGE . . . . . . . . . . . . . . 114

5.4.5.2 Seminative PAGE . . . . . . . . . . . . . . . 115

5.5 Protein characterization . . . . . . . . . . . . . . . . 115

5.5.1 In vitro switching of fluorescent proteins . . . . . . . . . . . . . . 115

5.5 .2 Metastability . . . . . . . . . . . . . . . . . 115

5.5 .3 Absorption spectra . . . . . . . . . . . . . . 116

5.5.4 Emission spectra of on- and off-states . . . . . . . . . . . . . 116

5.5.5 Extinction coefficient calculation . . . . . . . . . . . . . 116

5.5 .6 Quantum yield calculation . . . . . . . . . . . . . . . . 117

5.5.7 Molecular Brightness . . . . . . . . . . . . . . . . . . . . . . 117

5.5 .8 Fluorescence lifetime . . . . . . . . . . . . . . . . 117

5.5.9 pH dependent absorption, excitation and emission spectra . . . . . . 117

5.5 .10 Size exclusion chromatography . . . . . . . . . . . . . . . . . 118

5.6 Microscopy methods . . . . . . . . . . . . . . . . . . . . . . . . . . . . . 119

5.6 .1 Sample preparation . . . . . . . . . . . . . . . . 119

5.6.2 Optical setup of the RESOLFT microscope . . . . . . . . . . . . . 119

5.6 .3 Power calculation . . . . . . . . . . . . . . . . . . 120

5.6 .4 RESOLFT microscopy . . . . . . . . . . . . . . . . . . . . 121

5.6 .5 STED microscopy . . . . . . . . . . . . . . . . . . . . . . . . . . . . . . . . . . 122

5.6 .6 Image analysis . . . . . . . . . . . . . . . . . . . . . . . 122

6 Material 123

6.1 Buffer and media . . . . . . . . . . . . . . . . . . 123

6.2 Software . . . . . . . . . . . . . . . . . . . . 125

6.3 Primer . . . . . . . . . . . . . . . . . . . 125

7 Appendix 128

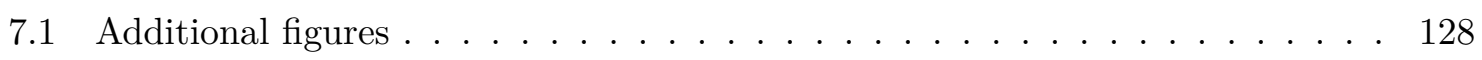

7.2 Additional tables . . . . . . . . . . . . . . . . . . 137 
8 List of figures $\quad 138$

9 List of tables $\quad 140$

10 References $\quad 141$

11 Acronyms $\quad 158$

12 Acknowledgements $\quad 160$

13 Curriculum vitae 162 


\section{Introduction}

\subsection{Microscopy}

The field of biology aims at understanding the fundamental mechanisms underlying life and nature. Since the first visualization of a plant cell with a microscope by Hooke in 1665 [1], cellular biology has focused on unraveling the elemental building blocks of life. Technical advances and improved optics allowed for the discovery of subcellular structures and compartments like the nucleus [2]. The discrimination of cellular features in brightfield microscopy is based on the contrast between structures resulting from their differing optical densities and scattering or absorption of passing light. However, molecular structures are not addressable with this approach.

\subsubsection{Fluorescence microscopy}

Fluorescence microscopy overcomes the limitations of brightfield microscopy and enables the selective visualization of molecular structures. Subcompartmental structures can be specifically labeled with fluorophores and allow for the discrimination of single molecules from the complex proteome present within a cell due to the fundamentally different principle of fluorescence microscopy compared to brightfield microscopy. Instead of the full visible spectrum (VIS) of light, fluorescence microscopy uses a narrow part of that spectrum or a single wavelength from a laser. The incident light is absorbed by fluorophores in the sample which transfers an electron from the ground-state $S_{0}$ to an excited state $S_{1}$. The energetic difference between $S_{0}$ and $S_{1}$ is equal to the energy $h \nu$ of the absorbed photon, where $h$ is Planck's constant and $\nu$ the frequency of light. The electron subsequently relaxes back to the ground-state, which occurs under the emission of a photon. Because the electron relaxes vibrationally within the excited state, the subsequent relaxation to the ground-state emits a photon of longer wavelength. This discrepancy between the absorbed energy and the emitted one is known as Stokes shift. The average time a fluorophore spends in the excited state before fluorescence emission is known as the fluorescence lifetime, which is in the range of a few nanoseconds. Naturally, there are other means of relaxation to the ground-state that are non-radiative such as vibrational relaxation, which is dissipated as heat within the sample. In addition, the excited state can be converted into a triplet-state $T_{1}$ via intersystem crossing. This state is stable for a prolonged time and can relax under the emission of a photon as well, an event which is known as phosphorescence. The ratio of photons emitted as fluorescence and photons absorbed is defined as the fluorescence quantum yield, a distinctive characteristic of fluorophores.

Structures labeled with fluorescent tags (or 'dyes') facilitate optimal contrast in fluorescence microscopy as the excitation light can be blocked by a filter and all light recorded by a camera during imaging originates from such structures, whereas the remaining areas of a cell ideally emit no light at all. However, in widefield microscopy of living cells this contrast is attenuated 


\section{Introduction}

by out-of-focus light. Because the whole specimen is illuminated, fluorescence emission originates not only from the focal plane of the objective lens but also from out-of-focus planes. This results in a blurred image as the resulting image planes are not aligned with the camera (fig. 1.1a). Fine structures in a densely labeled cell are thus masked.
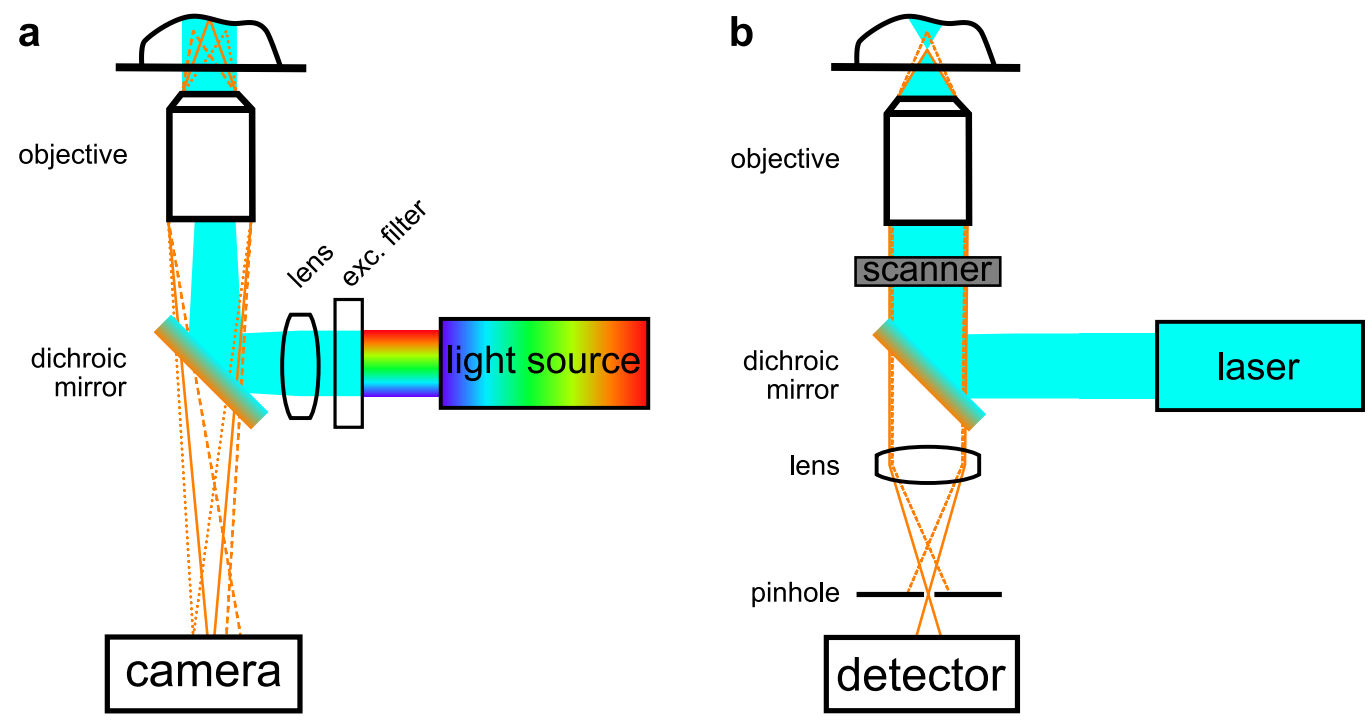

Figure 1.1: Principle of widefield and confocal fluorescence microscopy. (a) Widefield fluorescence microscope. The light source provides light that covers the VIS spectrum, the excitation light is selected with the excitation (exc.) filter. The whole sample is illuminated in the field of view and fluorescence is recorded with a camera from all layers. (b) Confocal laser scanning microscope. Fluorescence excitation is facilitated with light from a laser source. The collimated beam is focused to a diffraction-limited spot in the sample and deflected by a scanner to scan the sample. Emitted fluorescence is focused on a detector and out-of-focus light is blocked by a pinhole, ensuring that the fluorescence signal recorded emerges from the focal plane.

This problem was solved with the development of the confocal laser scanning microscope (fig. 1.1b). Instead of illumination of the whole specimen, the excitation light from a laser enters the objective lens as a collimated beam and is thus focused to a single spot, which limits and defines the excited volume in the focal plane. The focal spot is moved through the sample by means of a deflecting scanner. At every coordinate in the focal plane the fluorescence signal is recorded by a detector such as a photomultiplier tube (PMT) or an avalanche photodiode (APD) to reconstruct the image pixel by pixel. Repeating this process in different focal planes allows for three-dimensional sectioning of the sample as light above and below the focal spot is blocked by a pinhole in front of the detector. Because the image is reconstructed by scanning the sample, this method requires more time for recording a single frame compared to a widefield microscope equipped with a camera, but limiting the origin of fluorescence emission to a single plane greatly improves the contrast. In addition, the precise spatial control over the incident light allows for experiments like FRAP (fluorescence recovery after photobleaching) in spatially defined subcelluar volumes (reviewed in [3]) or imaging of complex three-dimensional samples. 
Practically, the contrast in fluorescence microscopy is influenced by the following aspects: Depending on the chosen fluorophore and its spectral properties, the excitation light evokes autofluorescence, which occurs when the absorption spectrum of the fluorophore overlaps with that of cellular molecules capable of fluorescence emission. This problem becomes more severe with wavelengths like $405 \mathrm{~nm}$, which are close to the ultraviolet (UV) spectral range where excitation of biological molecules like nicotinamid adenine dinucleotide (NADH), flavine adenine dinucleotide (FAD), or porphyrins causes autofluorescence [4]. It can be overcome by the implementation of fluorescence lifetime imaging (FLIM), a method in which differing lifetimes of fluorophore labels and cellular components contributing to autofluorescence are used to discern both emitters $[5,6]$. With lifetimes in the nanosecond range, excitation with a pulsed laser can be used to determine the delay between fluorescence excitation and arrival of emitted photons at the detector. If the lifetime of the autofluorescent component and the label are sufficiently different, photons can be sorted into two channels, effectively eliminating autofluorescence from the label channel. This has for example been demonstrated in planta with chloroplasts [7], which particularly contribute to autofluorescence in plant cells [8,9], and citrine, a genetically encoded fluorescent protein [10]. Naturally, FLIM also allows for multi-color imaging with multiple dyes that cannot be separated spectrally [5].

Another approach to eliminate autofluorescence is to avoid the spectral range in which it occurs. This can be achieved with multi-photon excitation of fluorophores [11] or by shifting their excitation and emission to regions beyond the VIS range. Whereas the human eye is unresponsive to these wavelengths, cameras can detect photons emitted in the near infrared (NIR) region of $650-900 \mathrm{~nm}$. Here, tissue absorption by $\mathrm{H}_{2} \mathrm{O}$ or hemoglobin is minimal and thus results in lower autofluorescence [12]. In addition, the use of wavelengths in the NIR region has further advantages for imaging of more complex samples such as tissue of live organisms. At higher imaging depths, NIR light is less attenuated and scattered [13], and the point spread function (PSF) of the emitting fluorophores is less prone to aberrations in comparison to UV/VIS light [14]. A longer wavelength, however, has implications for the achievable resolution, which is based on the diffraction barrier of light.

\subsubsection{Diffraction barrier in light microscopy}

Although fluorescence microscopy enhanced the contrast of biological samples and technical advances improved the optical components of microscopes, resolving of fine details was still limited. In a widefield microscope, the resolution is determined by the quality of the objective and the pixel size of the camera, but the resolution cannot be improved infinitely by optimizing the components due to fundamental physical properties of light. Confocal microscopes reconstruct the image based on the intensity of a fluorescence signal and the known coordinate of the focal spot. Consequently, the size of the far-field focal spot determines the excited volume and thus the resolution. The size of the focal spot, however, is dependent on the wavelength of the focused light. This relation was first stated by Ernst Abbe in 1873 [15]. The size of the focal spot in lateral and axial dimension can be described as follows: 


\section{Introduction}

Lateral resolution:

$$
r_{\text {lateral }}=\frac{\lambda}{2 n \sin \alpha}
$$

Axial resolution:

$$
r_{\text {axial }}=\frac{\lambda}{n \sin ^{2} \alpha},
$$

where $\lambda$ is the wavelength of light, $n$ the refrative index of the immersion medium, and $\alpha$ the half-angle of the aperture of the objective lens.

The factor $n \sin \alpha$ is known as the numerical aperture (NA) and a property of the objective design. Therefore, the achievable resolution is limited by the wavelength and NA. This limitation is known as the diffraction barrier in microscopy, as both the numerical aperture and the employable wavelength of the excitation light cannot be optimized infinitely in light microscopy.

Another way to define resolution in fluorescent samples is the minimal distance $d$ required for two infinitely small emitters at which they still can be discerned. A single emitter of this kind results in an image with a circular intensity peak that is surrounded by several other, ring-like intensity maxima and minima known as Airy pattern [16]. The radius of the first minimum around the center is the minimal distance $d$ two identical of these emitters must have in order to be distinguishable. This measure of resolution is known as the Rayleigh criterion [17]:

Lateral resolution:

$$
d_{\text {lateral }}=0.61 \frac{\lambda}{\mathrm{NA}}
$$

Axial resolution:

$$
d_{\text {axial }}=\frac{2 n \lambda}{\mathrm{NA}^{2}}
$$

In practical applications, the NA of the objective typically has a maximal value of approximately 1.4. With an emission wavelength of $500 \mathrm{~nm}$, this results in an approximate resolution of $200 \mathrm{~nm}$ laterally and $750 \mathrm{~nm}$ axially, which is often estimated by the full-width half-maximum (FWHM) of a Gaussian or Lorentzian function fitted to the intensity cross section of structures in microscopy images. Due to the dependency on the wavelength, light in the NIR region results in a lower resolution, while shorter wavelengths improve the resolution. This dependency has been utilized with scanning electron microscopy (reviewed in [18]), where the wave nature of electrons allows to achieve resolutions on the atomic 
level [19]. Unfortunately, sample preparation requires fixation and coating of structures with heavy metals, which excludes live-cell specimens from this approach.

In a confocal laser scanning microscope, the resolution is theoretically improved in comparison to calculations based on Abbe's equation (eq. (1.2)) because the effective PSF results from the product of the excitation spot and the emission PSF. The resolution is thereby improved by a factor of $1 / \sqrt{2}$. However, this calculation is based on an infinitely small pinhole, which is not feasible because an increasingly smaller pinhole attenuates the fluorescence signal and thus impairs the signal-to-noise ratio. Practically, the resolution of a confocal microscope is therefore only minimally improved [20, 21]. Nevertheless, several methods to overcome the diffraction limit have been presented in the last two decades.

\subsubsection{Diffraction-unlimited microscopy}

Several concepts, collectively termed super-resolution microscopy, have been developed to achieve improved resolutions in the lateral and axial dimension (reviewed in [22]). 4Pi [23] and $\mathrm{I}^{5}$ microscopy [24] improve the axial resolution by employing coherent excitation and detection of fluorescence bilaterally with two objectives, which results in 3-4 times better axial resolution due to constructive interference and smaller effective PSFs of the incident and thus emitted light [25]. Structured illumination microscopy (SIM) [26] improves the resolution by a factor of 2 by reconstructing the spatial distribution of fluorophores from a series of images recorded with shifted structured illumination. Further methods include superresolution optical fluctuation imaging (SOFI) [27], AiryScan [28], expansion microscopy [29], and lattice light sheet microscopy [30]. However, these methods improved the resolution and/or contrast, but were ultimately still diffraction-limited. Other methods on the other hand, which are summarized under the term nanoscopy, facilitate diffraction-unlimited superresolution microscopy and in theory allow for an infinite improvement of the resolution. These methods utilize discernible states of fluorophores such as a fluorescent on-state and a non-fluorescent off-state to achieve improved super-resolution microscopy of fluorescently labeled structures. Two categories of nanoscopy can be distinguished: coordinate-targeted and coordinate-stochastic approaches [22]. Both categories include multiple realizations of nanoscopy concepts and differ in how fluorescence of a sub-diffraction sized volume is mapped to the respective coordinate of the final image (fig. 1.2). The following sections will give an overview over the demonstrated approaches of nanoscopy.

\subsubsection{Coordinate-stochastic nanoscopy}

Concepts of coordinate-stochastic nanoscopy rely on the localization of single fluorescence emitters. Their fluorescence is recorded in a widefield microscope with a camera, which results in a diffraction-limited spot in the image (fig. 1.2c). However, this spot can be mathematically fitted to determine its centroid, which allows for the localization of the origin of fluorescence, that is, the fluorophore. Precision of this localization is dependent on the num- 


\section{Introduction}

ber of photons $N$ and scales with $1 / \sqrt{N}$. Thus, a high bleaching resistance is a requirement for fluorophores in order to obtain as much photons as possible. An infinite number of photons emitted from a single fluorophore would allow for infinitely precise localization of this emitter, rendering this approach diffraction-unlimited.

For the imaging of biological specimens, thousands of single molecule fluorescence events of fluorophores labeling the desired structure are recorded. They are subsequently localized, and the spatial information obtained is used to reconstruct the nanoscopic image, the emitters are thus discerned in the temporal dimension. The critical requirement here is that localization is performed on isolated, single emitters. Whereas a single frame can of course be used to locate multiple emitters, these are required to be spatially separated by a sufficient distance. Because the single frames recorded are diffraction-limited, two emitters that are separated by a distance smaller than $r_{\text {lateral }}$ (eq. (1.1)) are indiscernible [21]. Consequently, coordinate-stochastic methods rely on fluorophores to be distinguishable by different states, which is mostly realized with a non-fluorescent off- and a fluorescent on-state.

The concept described above was first demonstrated in 2006 in a number of independent publications under the names (fluorescence) photoactivated localization microscopy ((f)PALM) [31, 32] and stochastic optical reconstruction microscopy (STORM) [33]. (f)PALM utilizes photoactivatable fluorophores, which are non-fluorescent in their native state. Upon irradiation with a wavelength distinct from the fluorescence excitation wavelength, they are activated irreversibly to their fluorescent state. Low activation intensities ensure that only a small subset of emitters is active at a time, and these emitters are fully bleached during or after frame acquisition to avoid accumulation and subsequent overlapping of active fluorophores [31, 32]. STORM, on the other hand, uses photoswitchable dyes which can repeatedly be alternated between an on- and an off-state with light of different wavelengths [33]. In these concepts, the number of fluorophores in the fluorescent state is regulated by the intensity of the incident light, and the activation is not spatially controlled but occurs randomly in the illuminated field of view. Thus, the term coordinate-stochastic nanoscopy. The initial demonstrations discussed above could resolve structures with $20 \mathrm{~nm}$ resolution, but image acquisition time was in the range of multiple hours, which prevents their application for imaging of fast cellular dynamics [31, 33]. Shortly after, the (f)PALM/STORM protocol was accelerated to the range of minutes with PALM with independently running acquisition (PALMIRA) [34-36]. Image acquisition is in this case not synchronized to sequential activation and deactivation. Instead, a reversibly switchable fluorophore is driven to the off-state with the excitation laser and the return of single emitters to the on-state occurs spontaneously while images are recorded independently. This approach is similar to ground-state depletion microscopy followed by individual molecule return (GSDIM) [37], or direct STORM (dSTORM) [38], where the majority of fluorophores is driven into a longlived dark-state, such as the triplet-state $\mathrm{T}_{1}$, with high excitation laser intensities. Individual emitters are imaged upon their return from the dark-state and allow for their localization. Another implementation of discernible fluorescence states is points accumulation for imaging in nanoscale topography (PAINT). Here, fluorophores can reversibly transition between 
being bound to the target structure and an unbound state with free diffusion. Fluorescence emitted from the former immobilized state can be localized [39]. All nanoscopy concepts described above rely on distinguishing single, stochastically active emitters. This is different from coordinate-targeted methods, where the fluorescent state is confined spatially.

a

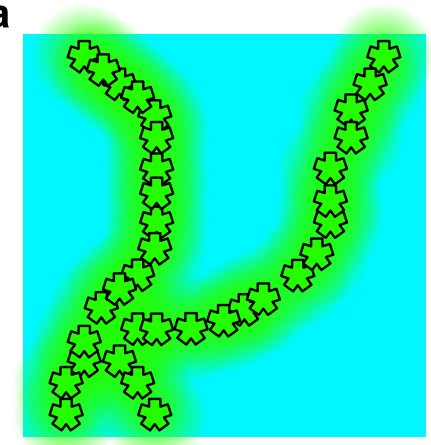

b

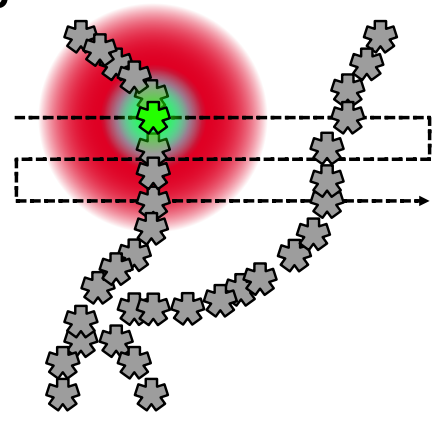

C

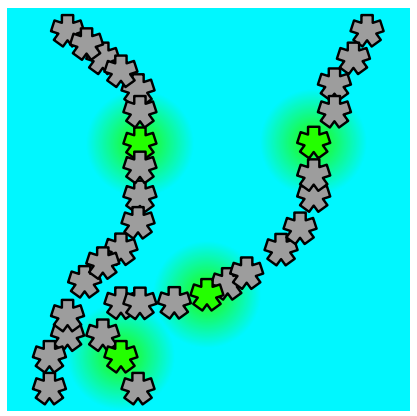

Figure 1.2: Principle concepts of far-field nanoscopy. (a) Widefield (or confocal, not illustrated) excitation of fluorophores (green asterisks) labeling a structure results in a diffraction-limited image with overlapping emission PSFs. (b) Coordinate-targeted nanoscopy utilizes a light pattern like a doughnut-shaped beam to spatially restrict fluorescence emission.

(c) Coordinate-stochastic nanoscopy localizes the centroid of the diffraction-limited spot of single emitters.

\subsubsection{Coordinate-targeted nanoscopy}

Coordinate-targeted nanoscopy employs a more direct approach to localize fluorescent emitters with sub-diffraction precision. The concept was first proposed in 1994 [40-42] and uses a spatial light pattern to facilitate saturated transition of molecules between states (fig. 1.2b). It was first realized in 2000 with the demonstration of stimulated emission depletion (STED) microscopy [41], an approach which uses a doughnut-shaped focal spot with a central region of zero intensity to laterally render peripheral fluorophores non-fluorescent (although in principle any pattern with a zero-intensity region can be used and the concept naturally can also be applied in the z-dimension). This is achieved by stimulated emission depletion of the excited state $S_{1}$. Fluorophores in this state can be depleted to $S_{0}$ in a process driven by a second red-shifted depletion laser (fig. 1.3), which stimulates the relaxation of $\mathrm{S}_{1}$. The photon emitted as a result of stimulated emission is red-shifted in comparison to the photon emitted as a result of spontaneous fluorescence and can thus be separated from it [43].

However, this process competes with the spontaneous emission of fluorescence, which occurs in the nanosecond timescale. To fully prevent spontaneous fluorescence emission and to saturate STED, the second laser thus has to be applied with a high intensity in the range of $\mathrm{MW} / \mathrm{cm}^{2}$ and a short pulse duration much smaller than the fluorescence lifetime [44]. Both the excitation and depletion laser are usually pulsed in applications of STED microscopy, with the delay and wavelength necessary for optimal STED being a property of the dye.

Due to the doughnut-like shape of the depletion laser, the required intensity for STED is 


\section{Introduction}
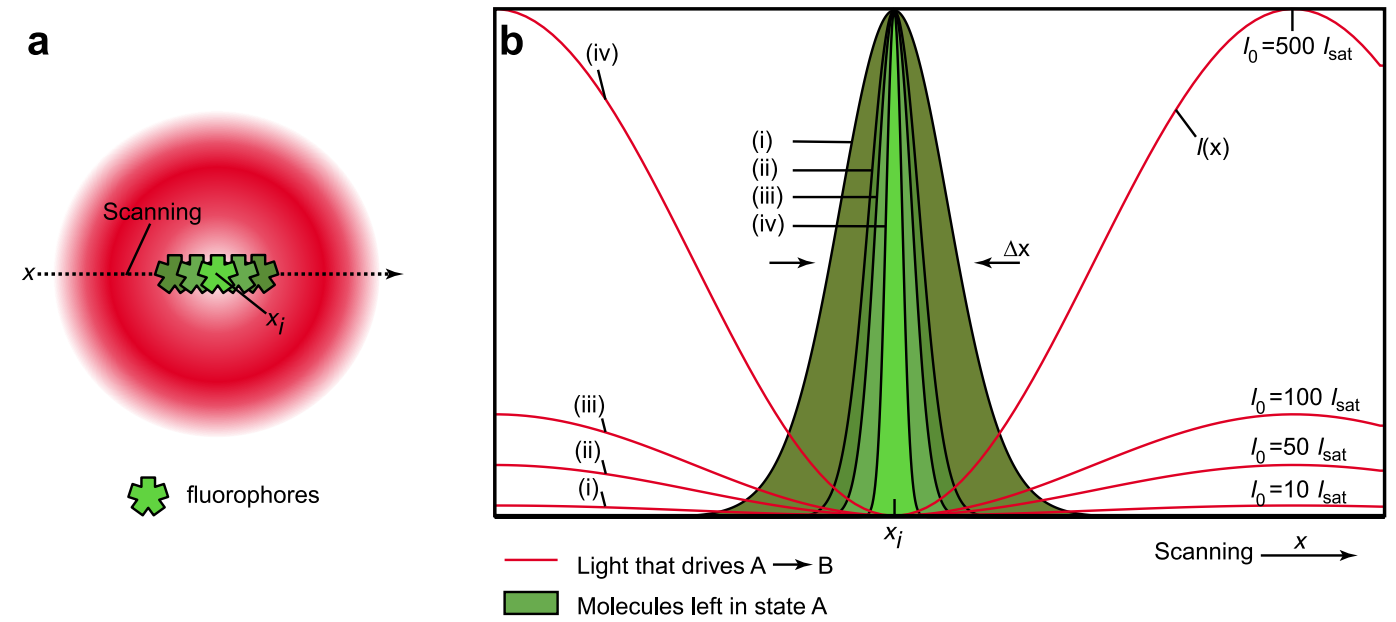

Figure 1.3: Stimulated emission depletion. (a) The doughnut-shaped stimulated emission depletion beam scans the sample during imaging with a centrally aligned Gaussianshaped excitation beam. (b) High STED intensities with $I \gg I_{\text {sat }}$ deplete fluorophores in the fluorescent state A to the non-fluorescent state B, resulting in a smaller effective PSF (green profiles). Red graphs represent the intensity profile of the STED spot along the scanning axis $\mathrm{x} . \quad I_{\text {sat }}$ is the intensity at which half of the fluorophores are depleted. Figure b was adapted from [44], with permission from Elsevier, () 2004.

only achieved for peripheral fluorophores, and the central region remains fluorescent. With higher intensities of the depletion laser, this central region becomes smaller. The achievable resolution can be approximated in the lateral dimension based on equation (1.1) [21]:

$$
\Delta r \approx \frac{\lambda}{2 n \sin \alpha \sqrt{1+I_{\max } / I_{\mathrm{sat}}}}
$$

where $n$ is the refrative index of the immersion medium, $\alpha$ the half-angle of the aperture of the objective, $I_{\max }$ the peak intensity of the depletion laser, and $I_{\text {sat }}$ the intensity at which half of the fluorophores are depleted from $S_{1}$ to $S_{0}$.

The resolution thus scales with $1 / \sqrt{1+I_{\max } / I_{\text {sat }}}$ and is diffraction-unlimited for infinitely high $I_{\max }$ intensities; high doughnut intensities and low saturation intensities for STED result in a small central region where fluorophores remain fluorescent. Because this approach employs scanning of the sample with a STED laser and an excitation beam, the sub-diffraction sized volume of emitting fluorophores can be directly mapped to the respective coordinate (or pixel) to reconstruct the image, much like in confocal laser scanning microscopy. Compared to coordinate-stochastic methods this approach has thus the advantage that no calculations are required after data has been recorded.

Recently, the advantages of coordinate-targeted and coordinate-stochastic methods have been combined to achieve a quasi-molecular resolution of $1 \mathrm{~nm}$ [45]. Nanoscopy with minimal photon fluxes (MINFLUX) achieves such precise localization by employing a patterned 
illumination to determine the position of single, stochastically active emitters. Similar to coordinate-targeted approaches, the pattern has a region of zero intensity and probes fluorescence of the emitter at different positions. The exact position can then be calculated based on the intensity distribution within the pattern and the fluorescence intensity of the probing steps, which requires much less photons than coordinate-stochastic methods [22, 45].

Other concepts than STED have been proposed and realized to separate different states of fluorophores for coordinate-targeted nanoscopy. Ground-state depletion (GSD) microscopy uses patterned illumination to pump peripheral fluorophores into a long-lived dark state such as the triplet-state $[21,46,47]$. While the term RESOLFT, which stands for reversible saturable optically linear fluorescence transition, denotes the general concept of coordinate-targeted nanoscopy methods [44, 48], it is mostly used for coordinate-targeted nanoscopy concepts which employ reversibly switchable fluorophores for the distinguishment of states [22]. While these can be organic dyes $[49,50]$, the first demonstration was with a switchable fluorescent protein [51]. The following sections will give an overview over fluorescent proteins and their switching capabilities before their application and requirements for RESOLFT nanoscopy will be discussed. Further use of the acronym RESOLFT will refer to the utilization of switchable fluorophores.

\subsection{Fluorescent proteins}

Fluorescent proteins have revolutionized live-cell microscopy as they provide a simple way to tag subcompartmental structures. While staining of such structures can be facilitated at high specificity with antibodies labeled with organic dyes, the protocols required for intracellular target proteins such as fixation or permeabilization preclude their application in living cells or whole organisms. Fluorescent proteins, on the other hand, can easily be fused to the desired target protein. After full maturation, these proteins exhibit fluorescence, which can be utilized for diffraction-limited or -unlimited microscopy.

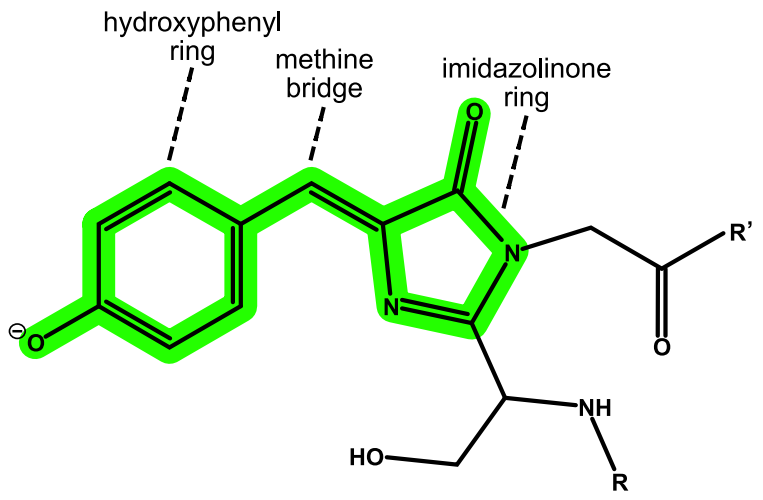

Figure 1.4: Chromophore structure of avGFP. The 4-( $p$-hydroxybenzylidene)-5imidazolinone chromophore of avGFP is formed autocatalytically from the tripeptide Ser65Tyr66-Gly67 in the central $\alpha$-helix of the $\beta$-barrel [52-54]. The formation of the methine bridge, which connects both aromatic rings and shifts the absorption of avGFP to the VIS range, requires oxidation by molecular oxygen [55]. 


\section{Introduction}

The first description of a fluorescent protein was published in 1962 by Shimomura etal., who discovered a protein that was responsible for green fluorescence of samples obtained from the jellyfish Aequorea victoria [56]. This protein was later termed green fluorescent protein (GFP, or avGFP), and its biological function is to red-shift the apparent wavelength of bioluminescence emitted from aequorin [57]. Despite its early description, the scientific impact of avGFP was low in the following years. This changed when its cDNA was cloned in 1992 by Prasheretal., revealing the genomic sequence of avGFP, which codes for the protein of 238 amino acids with a molecular weight of $27 \mathrm{kDa}$ [52]. Soon after, the protein was expressed in E. coli and proposed as a marker for gene expression as it could be shown that maturation towards the fluorescent state of avGFP required no external factors in a cellular context except for the presence of oxygen [58-60].

a

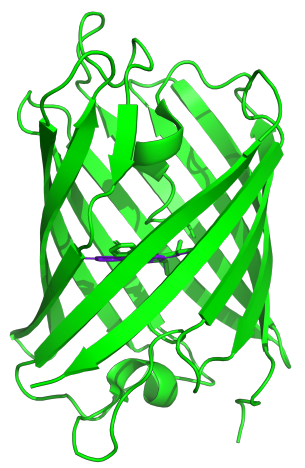

b

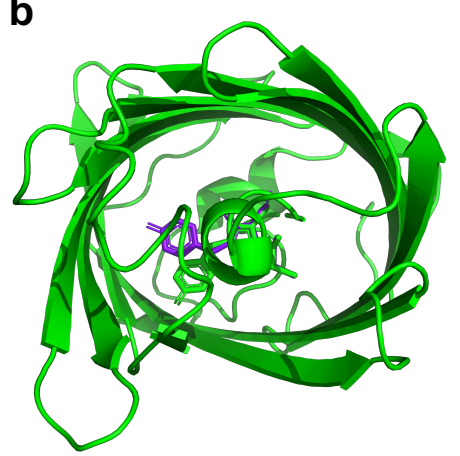

C

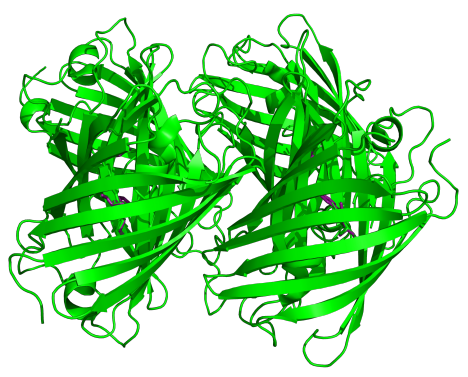

Figure 1.5: Crystallographic $\beta$-barrel structure of Padron. (a) Side view, (b) top view, and (c) tetrameric interaction of the crystal structure are shown in cartoon representation. The chromophore is in stick representation, with the on-state in green and the off state in purple (PDB IDs 3ZUJ and 3ZUF, [61]).

Already in 1978 the fluorescent chromophore was proposed to be a 4-( $p$-hydroxybenzylidene)5-imidazolinone moiety integrated into the backbone of the protein (fig. 1.4, [62]). The crystal structure was solved in 1996 and revealed a barrel-like structure built from $11 \beta$-sheets with a central $\alpha$-helix, which was termed $\beta$-barrel [63, 64]. Figure 1.5 depicts the $\beta$-barrel structure of Padron. Since then, a large number of homologues have been found in a variety of organisms covering a large range of spectral properties [65-68]. Due to the similarity of overall structures and chromophores within this family of proteins, they are often referred to as GFP-like proteins. Other fluorescent proteins with different fluorogenic moieties exist, but will only be briefly mentioned in the following sections as the focus of this work is on the GFP-like protein Padron.

\subsubsection{Chromophore maturation and spectral properties of GFP}

Although sequence identity of GFP-like fluorescent proteins can be as low as $23 \%$ [69], all proteins in this class fold into a common barrel-like structure. This structure is a prerequisite for the fluorogenic properties of these proteins: Early experiments with synthetic compounds 
identical to the chromophore moiety resulted in similar absorption as observed in denatured avGFP [70], but displayed no fluorescence emission [71]. Lowering the temperature to $77 \mathrm{~K}$, on the other hand, enabled fluorescence in the synthetic chromophore, which was attributed to the chromophore microenvironment and a lack of isomerization around the methine bridge [71]. The importance of the chromophore surroundings in the protein were supported with later research elucidating the origin of this moiety (reviewed in [72]). The fluorogenic moiety is formed after proper folding in an autocatalytic process from a tripeptide motif situated in the central $\alpha$-helix, involving Ser65, Tyr66, and Gly67 [52-54]. The initial step here is a cyclization of the helix backbone, where the peptide bond nitrogen of Gly67 performs a nucleophilic attack on the carbonyl group of Ser65, forming an intermediate with a heterocycle consisting of the Ser65 carbonyl group carbon, Tyr66 nitrogen, $\mathrm{C}_{\alpha}$, and carbonyl carbon, as well as Gly67 nitrogen [60,73]. The elimination of water results in the aromatization of this formation and yields the imidazolinone ring [74], followed by oxidation with hydrogen peroxide as a byproduct, which is the rate-limiting step [55]. The latter reaction connects the conjugated $\pi$-systems of the Tyr66 hydroxyphenyl ring and the imidazolinone, shifting the absorption of the chromophore to the VIS range (fig. 1.4). The rate of this final, oxygen-dependent step differs between proteins and is often determined to compare maturation speed of variants. To do so, proteins can be expressed under anaerobic conditions and fluorescence increase as a result of maturation is then monitored at aerobic conditions. While the maturation time constant for GFP was determined to be 120 minutes, this value was reduced to 27 minutes in GFP-S65T [60, 75].

A prerequisite for chromophore formation is the spatial organization of the secondary structure within the barrel, which positions the autocatalytic tripeptide in the necessary orientation [76]. Configurations contributing are a certain lack of hydrogen bonds within the central helix, a backbone bend in the chromophore vicinity which positions the tripeptide, and the stabilization by amino acids such as Arg96 and Glu222, which are conserved and essential to chromophore formation [72, 76, 77]. The essential role of Arg96 in chromophore maturation is likely the reason for the strong conservation of Gly67, as larger amino acids would disturb the hydrogen bonding network due to steric hindrance resulting in impaired maturation $[72,77]$. The first GFP-like protein with an altered amino acid at this position, namely alanine, was published in 2016 [78]. The surrounding amino acids in the chromophore cavity have also been proposed to have a catalytic role. Arg96 lowers the energetic barrier for the initial cyclization of the backbone [76, 79], whereas Glu222 stabilizes deprotonation in intermediate steps of the reaction [80].

Aside from catalytic contribution, the amino acids in the chromophore pocket also influence the spectral properties of fluorescent proteins [81]. The wild-type GFP has an absorption spectrum with two distinct absorption peaks at 395 and $475 \mathrm{~nm}$, which result in fluorescence emission with a wavelength of 508 and $503 \mathrm{~nm}$, respectively [60]. The absorption peaks represent the protonation state of the hydroxybenzylidene moiety. While the peak at $395 \mathrm{~nm}$ is attributed to the protonated form of the chromophore, deprotonation results in an increased delocalization of electrons in the conjugated $\pi$-system and the concomitant 


\section{Introduction}

rise of the red-shifted absorption peak [82]. For avGFP, the Stokes shift of the emission in response to excitation in the UV range is particularly large; it results from a process termed excited state proton transfer (ESPT). The terminal proton of the hydroxyphenyl group is stabilized by the anionic Glu222. Upon excitation, it is fully transferred to Glu222 within the timescale of the excited state, giving rise to the phenolate form of the chromophore from which emission occurs [83-85]. The proton transfer is quickly reversed in avGFP, but repeated excitation can pump proteins into a state where the chromophore is fully deprotonated [83, 86], shifting its absorption towards the peak at $475 \mathrm{~nm}$. Conversely, S65T results in permanent deprotonation of the chromophore due to altered hydrogen bonds preventing ionization of Glu222 [84].

\subsubsection{Spectral variation of GFP-like proteins}

The autocatalytic maturation dynamics described in the previous section were reported for wild-type GFP with the chromophore shown in figure 1.4. However, in the years following its rediscovery, a plethora of fluorescent proteins have been reported covering the whole VIS range and extending into the NIR region [87-89], and recently the de novo design of a $\beta$-barrel capable of binding free chromophore to fluoresce has been demonstrated [90]. The FPbase, an open-source online resource for fluorescent proteins, lists 717 entries at the time of writing this thesis [67], figure 1.6 depicts their spectral variation. Because the database was created in 2018 and is based on contributions by the community, the actual number of fluorescent proteins published or discussed is likely to be even higher considering variants that have not yet been entered.

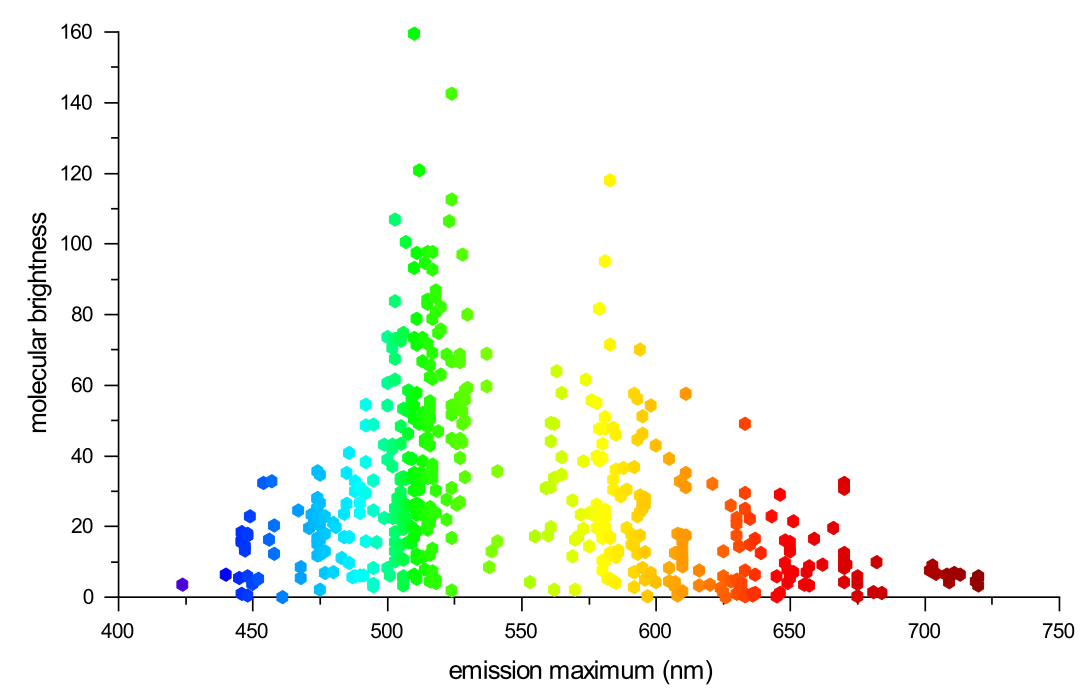

Figure 1.6: The spectral range of fluorescent proteins. The figure depicts the molecular brightness, which is calculated as the product of the extinction coefficient $\varepsilon$ and the quantum yield, in relation to the emission maximum. Values were taken from FPbase.org, where 486 of the 717 entries listed both molecular brightness and emission maximum at the time of writing this thesis [67]. 
A number of strategies have been found and utilized to increase the spectral range, the most obvious being to alternate the extent of the conjugated $\pi$-system. Obviously, a change in the $\pi$-system can be achieved by site-directed mutagenesis. While glycine in the chromophoreforming tripeptide is conserved to a large extent [78], it seems promising to mutate tyrosine, which contributes to the size of the $\pi$-system due to its aromatic nature. Consequently, it was replaced with tryptophane, histidine, and phenylalanine, all of which resulted in blueshifted variants emitting blue or cyan light [85, 91, 92]. Alternatively, the $\pi$-system can be influenced by the introduction of another aromatic residue in the vicinity of the chromophore which interacts with the phenolate moiety via $\pi$-stacking, thereby lowering the excitation energy due to increased polarity of the chromophore [93].

Naturally, shifting the absorption and emission to longer wavelengths compared to GFP in order to increase biocompatibility of the protein with live-cell imaging applications is desirable and can be achieved by extending the conjugated $\pi$-system. The first red-emitting GFP-like protein published was DsRed, which was isolated from the coral Discosoma sp. [94]. It has a conjugated $\pi$-system that extends beyond the $\mathrm{C}_{\alpha}$ of the first tripeptide amino acid participating in cyclization [69], which is generated in a second oxidation step that creates a blue intermediate and subsequently matures via hydroxylation and elimination of a water molecule to its final red form [95, 96]. DsRed, which forms stable tetramers, was later monomerized and published as mRFP1 with the 'm' denoting the monomeric state [97]. This variant was the template for an extensive mutagenesis study resulting in a set of 8 new red fluorescent proteins collectively denoted as mFruits, which displayed different hues in the red VIS range [98]. The underlying mechanisms that resulted in their shifted emission spectra were elucidated by Shu etal. [99]. They found that mOrange exhibits a further covalent modification introducing a third heterocycle to the backbone, which resulted in blue-shifted emission due to the removal of a carbonyl moiety from the $\pi$-system. Conversely, mStrawberry and mCherry were proposed to be red-shifted as a result of an altered electron density distribution resulting from changed protonation equilibria and side chain rearrangements in the chromophore vicinity [99]. The microenvironment of the chromophore thus plays an important role in determining the spectral properties. In mPlum [100] a large Stokes shift of $60 \mathrm{~nm}$ is attributed to hydrogen bond modulation and relaxation of the excited chromophore prior to emission $[101,102]$.

Recently, the spectral range of fluorescent proteins has been extended to the NIR range with bacteriophytochromes (reviewed in [66]). These photoreceptors are inherently nonfluorescent, but could be engineered to emit fluorescence $[103,104]$. They require biliverdin as an external chromophore, which can be naturally produced from heme by heme-oxygenase 1 in mammals, allowing for the application of bacteriophytochromes as a tag in organisms of this group. Alternatively, biliverdin can be added externally to a respective sample [66]. A distinct extension of the chromophore $\pi$-system is present in Kaede [105]. This protein exhibits green fluorescence in its native state, but can be converted to a red form with UV irradiation. Other proteins with this feature have been published after they were either cloned from cDNA or generated from constitutively fluorescent proteins [106-110]. They are collec- 


\section{Introduction}

tively termed photoconvertible fluorescent proteins (PCFPs). In all PCFPs the conjugated $\pi$-system is extended in response to excitation, mostly with light in the UV range. In Kaede, the presence of histidine as the first amino acid of the chromophore-forming tripeptide is a prerequisite for its photoconversion. Here, UV excitation induces a cleavage of the backbone of the central $\alpha$-helix at the peptide bond involving the histidine amino group. A following elimination reaction connects the existing $\pi$-system of the chromophore with the histidine side chain by introducing an additional double bond [111].

Similar to PCFPs, photoactivatable fluorescent proteins (PAFPs) exhibit a modification in response to irradiation. These proteins, however, are non-fluorescent (or barely fluorescent) in their native dark state and are then converted to a fluorescent state [112-115]. The first representative of this group was PA-GFP (PA for photoactivatable) [112], which exhibits green fluorescence after activation. The underlying mechanism is a strongly impaired ESPT as a result of a $\mathrm{T} 203 \mathrm{H}$ mutation, which affects the Glu222 orientation so that the chromophore is mainly present in its neutral form, but ESPT rarely takes place. UV irradiation decarboxylates Glu222, resulting in deprotonation of the chromophore, which in turn exhibits fluorescence [116].

Both PCFPs and PAFPs are suitable probes for PALM nanoscopy [31, 32]. However, their conversion is irreversible, which prevents employment of their spectral states for coordinatetargeted nanoscopy. The discovery of reversibly switchable fluorescent proteins on the other hand allowed for the establishment of alternating states for RESOLFT microscopy with low laser intensities. The following section will give an overview over the origin and capabilities of reversibly switchable fluorescent proteins before their use in RESOLFT nanoscopy will be discussed.

\subsubsection{Reversibly switchable fluorescent proteins}

Reversibly switchable fluorescent proteins (RSFPs) are proteins which display a particular form of photochromism. They can be reversibly switched between a fluorescent on-state and a non-fluorescent off-state in a process that is driven by irradiation with two different wavelengths. Such a behavior had been observed in engineered variants of GFP, but transition between states was incomplete and resulted in low contrast [117]. The first protein capable of a relevant transition between states was asFP595 [118], which, in an ensemble of proteins, displays switching to the on-state with yellow light while simultaneously emitting fluorescence. Irradiation with blue light on the other hand reduces fluorescence emission by switching the protein to the off-state [51]. Because the same wavelength is capable of switching the protein to the on-state that also evokes fluorescence, asFP595 is categorized as a positively switching RSFP (fig. 1.7). However, low fluorescence intensity and its tetrameric interaction prevented wider use in cell biology $[51,118]$. Soon after, Dronpa was published as the first bright, monomeric RSFP with an opposing switching scheme [119]. The fluorescence excitation wavelength simultaneously switches the protein off while irradiation in the UV range reverses this effect, categorizing Dronpa as a negatively switching RSFP. 


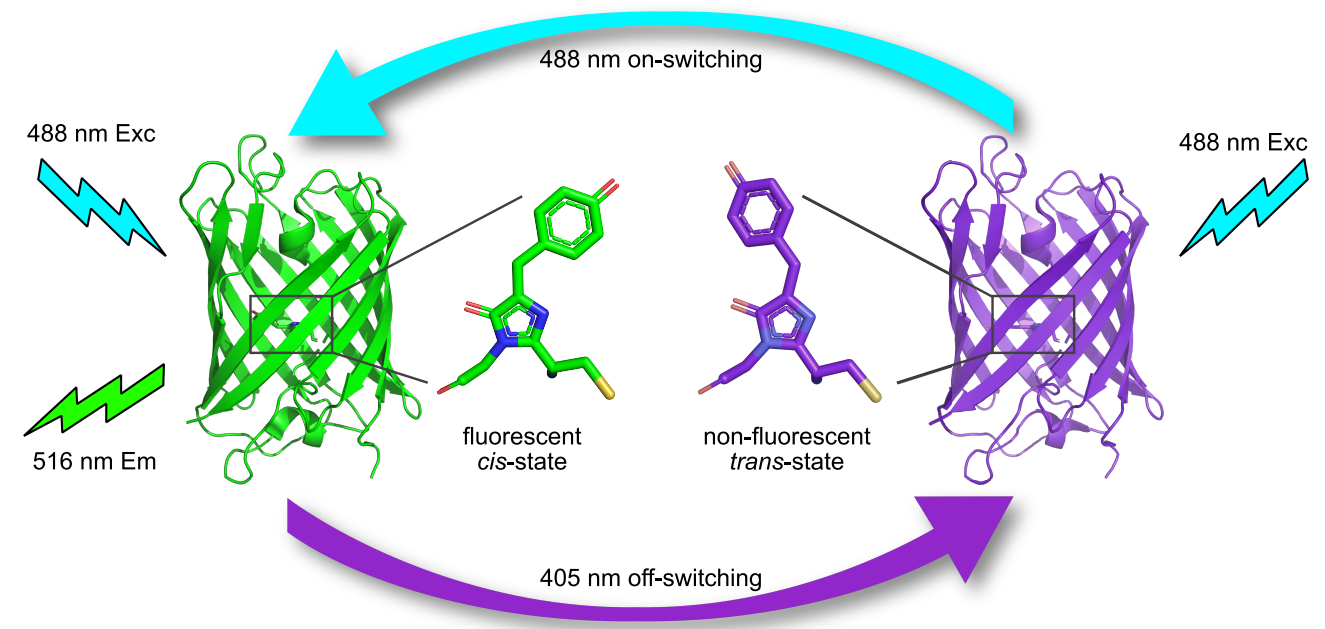

Figure 1.7: Reversible switching of Padron. Padron is a positively switching RSFP. This means that the excitation wavelength of $488 \mathrm{~nm}$ also induces cis-trans isomerization of the non-fluorescent trans-state (purple) to the fluorescent cis-state (green). The latter state can be reversed to the trans-state with light of $405 \mathrm{~nm}$.

So far, only few positively switching RSFPs have been reported, with asFP595 being the only wild-type form of these proteins. Others were: Padron [120], which was engineered from Dronpa, its successor Kohinoor [121], both emitting in the green VIS range, and rsCherry [122], which emits in the red VIS range. The majority of RSFPs are negatively switching RSFPs, which is why most research concerned with the elucidation of the switching mechanics has focused on these proteins. The group of negatively switchable RSFPs includes, among others: rsEGFP [123] and rsEGFP2 [124] as derivatives of GFP, DronpaM159T (or Dronpa-2) [125] and Dronpa-3 [126], which exhibit faster switching kinetics than Dronpa, Skylan-S [127] and Skylan-NS [128], two particularly bright and switchable variants based on EosFP [106], rsCherryRev [122], which was the first red-emitting negatively switching RSFP, as well as rsFusionRed2 and rsFusionRed3 [129], two proteins developed for RESOLFT nanoscopy. Two peculiar members of this group are NijiFP [130] and IrisFP [131], which can be reversibly switched but also maintain the ability of their respective template to be photoconverted from a green to a red fluorescent form.

\subsubsection{Switching mechanism of RSFPs}

Switching between states is facilitated by light induced isomerization of the chromophore as a result of a single-photon process $[61,132,133]$. Although the nature of this isomerization has been the focus of extensive research (reviewed in [81, 132]), the precise mechanism is still a subject of debate and might vary in different proteins. Both rotation around the bond between methine bridge and imidazolinone ring, which would require a spacious chromophore cavity, and a space-saving hula-twist have been found in rsEGFP2 [134].

While fluorescent proteins with a trans-chromophore have been described [135, 136], only one 


\section{Introduction}

RSFP with a fluorescent trans-state has been found so far [137]. All other RSFPs described display an off-state with a trans-chromophore [117]. For Dronpa, it was shown that the isomerization is accompanied by extensive rearrangements in the chromophore pocket and by protonation of the trans-state after off-switching. In addition, the cis-conformation of the on-state is stabilized via hydrogen-bonding [125, 138, 139]. Conversely, the M159Y mutation in Padron was sufficient to reverse the switching regime by stabilizing the trans-chromophore, which is deprotonated, via hydrogen bonding $[120,140]$. The ability to fluoresce is thus not determined by the isomeric state of the chromophore, but rather by the resulting conditions of the respective microenvironment [117]. This is supported by the suggestion that the non-fluorescence of the free chromophore in solution stems from its ability to rapidly isomerize [141]. Less stabilization of the $\beta$-barrel was also described for the non-fluorescent off-state of Dronpa [142, 143], and motions in the chromophore pocket of the trans-state of Padron were suggested as an explanation of its non-fluorescence [61].

So far, only two proteins have been reported which pose an exception from the isomerization switching mechanism: Dreiklang [144] and Spoon [145], which was engineered from Dreiklang, both emitting yellow fluorescence. Both proteins can be excited with light of $510 \mathrm{~nm}$ and switched on with UV light in the range of $350 \mathrm{~nm}$. However, in Dreiklang and Spoon, fluorescence excitation does not switch the protein, their continuous fluorescence emission is only limited by photobleaching mechanisms. Nevertheless, they can be switched to a nonfluorescent off-state with light in the range of $400 \mathrm{~nm}$. The switching mechanism does not involve an isomerization of the chromophore but rather relies on the reversible hydration and dehydration of the chromophore, thereby disrupting the conjugated $\pi$-system. Due to the separation of switching and excitation wavelengths this mode of switching was denoted as decoupled switching.

The molecular basis for spectral properties and switching characteristics is highly diverse. Dronpa for example undergoes extensive reorganization of the chromophore cavity upon switching [125, 139], whereas the same motions are absent in Padron [61, 140]. However, the resulting macroscopic changes such as the decrease of fluorescence or absorption spectra can be easily monitored in order to compare switching capabilities of different proteins. The following chapter will define the terminology required to describe the switching characteristics of RSFPs.

\subsubsection{Switching characteristics}

In order to utilize RSFPs in coordinate-targeted nanoscopy, they are required to fulfill certain characteristics. While a low tendency to form oligomers (fig. 1.5c) is necessary to label cellular structures without affecting their organization in response to aggregation, switching characteristics influence the robustness and quality of imaging. These factors can be engineered by mutagenesis of proteins; a defined terminology is thus required to describe the effects of such mutagenesis, figure 1.8 gives an overview over the associated parameters. 

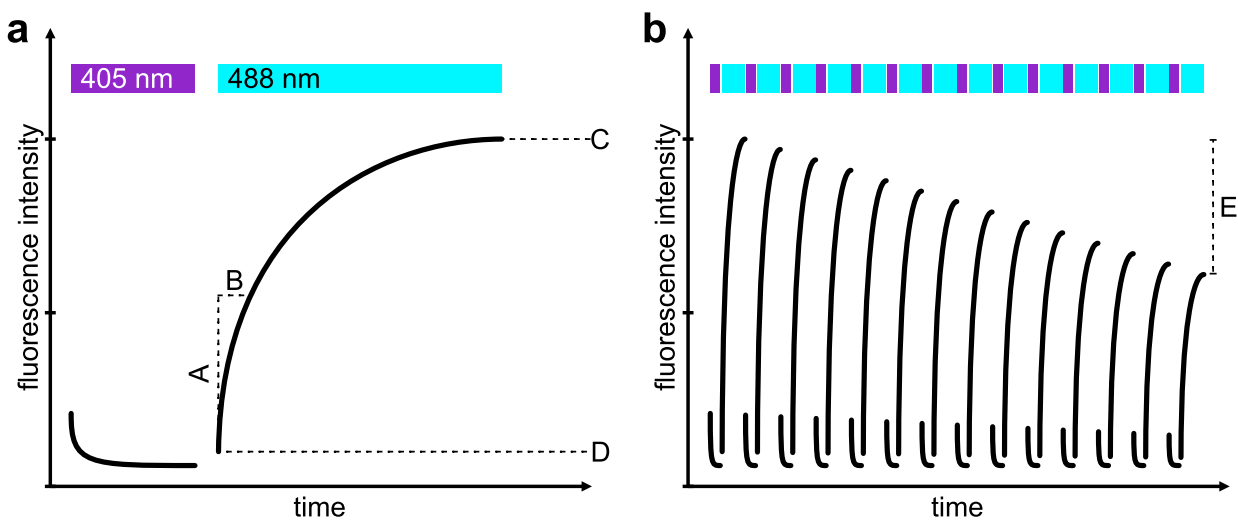

Figure 1.8: Switching characteristics of the positively switching RSFP Padron. (a) Single switching cycle. Light of $405 \mathrm{~nm}$ switches the protein to the off-state from which it can be returned to the on-state with $488 \mathrm{~nm}$. This step can be observed via fluorescence detection as an activation curve that allows for the calculation of characteristic switching parameters. The first data point $\mathrm{D}$ of the activation curve represents the off-state, while the protein has reached full on-state intensity $\mathrm{C}$ after activation. The ratio $\mathrm{D} / \mathrm{C}$ is the residual off-state fluorescence intensity. The half-time $\mathrm{B}$ is the time after which half of the ensemble fluorophores have reached the on-state $(\mathrm{A}=(\mathrm{C}-\mathrm{D}) / 2)$. (b) Multiple consecutive switching cycles. The decrease $\mathrm{E}$ of the on-state fluorescence intensity after a number of cycles is the switching fatigue and is reported as the intensity of the on-state of the last cycle relative to the one of the first cycle.

For any given sample, the fluorescence intensity (or brightness) can be observed rather easily (C in fig. 1.8a) with a suitable detector. While the molecular brightness is defined as the product of extinction coefficient $\varepsilon$ and the quantum yield, it has to be distinguished from the ensemble fluorescence intensity. While the former is a molecular entity, the latter is the apparent brightness of the sample. In the case of RSFPs, it is also influenced by the equilibrium of switching states, that is, the ratio of RSFPs that reside in the on- and off-state after thermal relaxation, which is denoted as the ground-state. The brightness is also influenced by external factors such as chromophore protonation and thus $\mathrm{pH}$, maturation, or temperature. It is therefore dependent on the experimental conditions as well as the sample, which is why interexperimental comparisons can be difficult [146, 147].

Upon deactivating RSFPs, an increasing number of proteins is present in the off-state. However, switching all RSFPs in an ensemble to one state or the other can be challenging. Processes such as thermal relaxation, spectral overlap of the on- and off-state, or return of proteins from a long-lived dark-state [148] influence the ratio of states. In addition, the configuration denoted as the off-state could still emit fluorescence depending on the fluorescence quantum yield of this state. RSFP ensembles thus display a residual off-state fluorescence intensity ( $\mathrm{D}$ in fig. 1.8a), which can be reported as the ratio of fluorescence intensity after off-switching and the fluorescence intensity in the on-state after full activation $(\mathrm{D} / \mathrm{C}$ in fig. 1.8a). The reciprocal value often is reported as the switching contrast [117]. In RESOLFT nanoscopy, this value determines how well states are separable, which is directly connected to the resolution improvement [149]. If the switching progress is monitored over time, these 


\section{Introduction}

values can easily be calculated from the switching curve (fig. 1.8a).

Likewise, the switching curve can be used for calculation of the ensemble switching half-time as a measure of switching speed (B in fig. 1.8a). Optimally, one would employ fitting of curves with a suitable mathematical model to reveal contribution of multiple factors. Alternatively, the switching half-time can be approximated by determining the time after which the fluorescence has increased (or decreased) by $50 \%$ of the difference between off- and on-state (time $\mathrm{B}$ to reach $\mathrm{A}=(\mathrm{C}-\mathrm{D}) / 2$ in fig. 1.8a). Switching speed is dependent on the light intensity as well as crosstalk between the on- and off-state at the given switching wavelength, and it is directly connected to the time required to record a RESOLFT image.

While photobleaching is associated with the irreversible fluorescence decrease in response to bleaching as a result of fluorescence excitation, switching contributes to the decrease of the ensemble brightness ( $\mathrm{E}$ in fig. 1.8b) as switching fatigue. It is reported either as the relative on-state fluorescence intensity after a given number of switching cycles or as the relative on-state fluorescence decrease per cycle. The ability to perform a large number of switching cycles is a necessity for RSFPs in RESOLFT nanoscopy [149], because every single fluorophore undergoes a multitude of cycles during point-scanning RESOLFT nanoscopy.

The parameters described here are intertwined to a large extent. Slowly switching RSFPs are more often excited during switching, resulting in a higher contribution of photobleaching to switching fatigue, while a low residual off-state fluorescence intensity renders the protein unresponsive to fluorescence excitation. If crosstalk between on- and off-states is relevant, a fast switching half-time for the activation could possibly prevent a low residual off-state fluorescence intensity if it cannot be compensated by the deactivation. If an RSFP is suitable for RESOLFT nanoscopy thus relies on the interplay and quality of these features.

\subsubsection{RSFPs in RESOLFT nanoscopy}

RESOLFT nanoscopy with reversibly switchable fluorescent proteins was proposed in 2003 [150] and first demonstrated with asFP595 in 2005 [51]. This demonstration was done with asFP595-filled grooves on a glass surface, because the tetrameric organization of asFP595 precluded it from correctly tagging live-cell target structures. Nevertheless, it performed well enough to show the potential of RESOLFT with sub-diffraction resolution at a low saturation intensity in the range of $\mathrm{mW} / \mathrm{cm}^{2}$, which is several orders of magnitude lower than the intensities typically employed for STED nanoscopy [51]. This is possible as the separation of discernible states is determined by the rate constants for on- and off-switching as well as their ability to reside in said states for a prolonged time relative to the pixel dwell time [51, 123]. In contrast, STED requires high laser intensities to deplete emission in the short-lived nanosecond timescale of the fluorescence lifetime [44]. RESOLFT microscopy with RSFPs is thus particularly suited for live-cell applications. This was first shown in 2011 with Dreiklang [144] and rsEGFP [123] by imaging cytoskeletal structures in cultivated cells, achieving a resolution as low as $35 \mathrm{~nm}$. While the conceptual realization of RESOLFT with asFP595 employed a deactivation pattern with a linear zero-intensity region with adjacent deactivating intensity 
peaks [51], the live-cell approaches used a doughnut-shaped deactivation pattern. In detail, fluorescence was activated in an initial step with a Gaussian-shaped beam and peripheral fluorophores were then switched to the off-state with the doughnut-shaped pattern. RSFPs remaining in the on-state were subsequently probed with a Gaussian-shaped readout laser. This sequence was repeated at every coordinate to reconstruct the image with sub-diffraction resolution. Figure 1.9 depicts this principle for a positively switching RSFP.
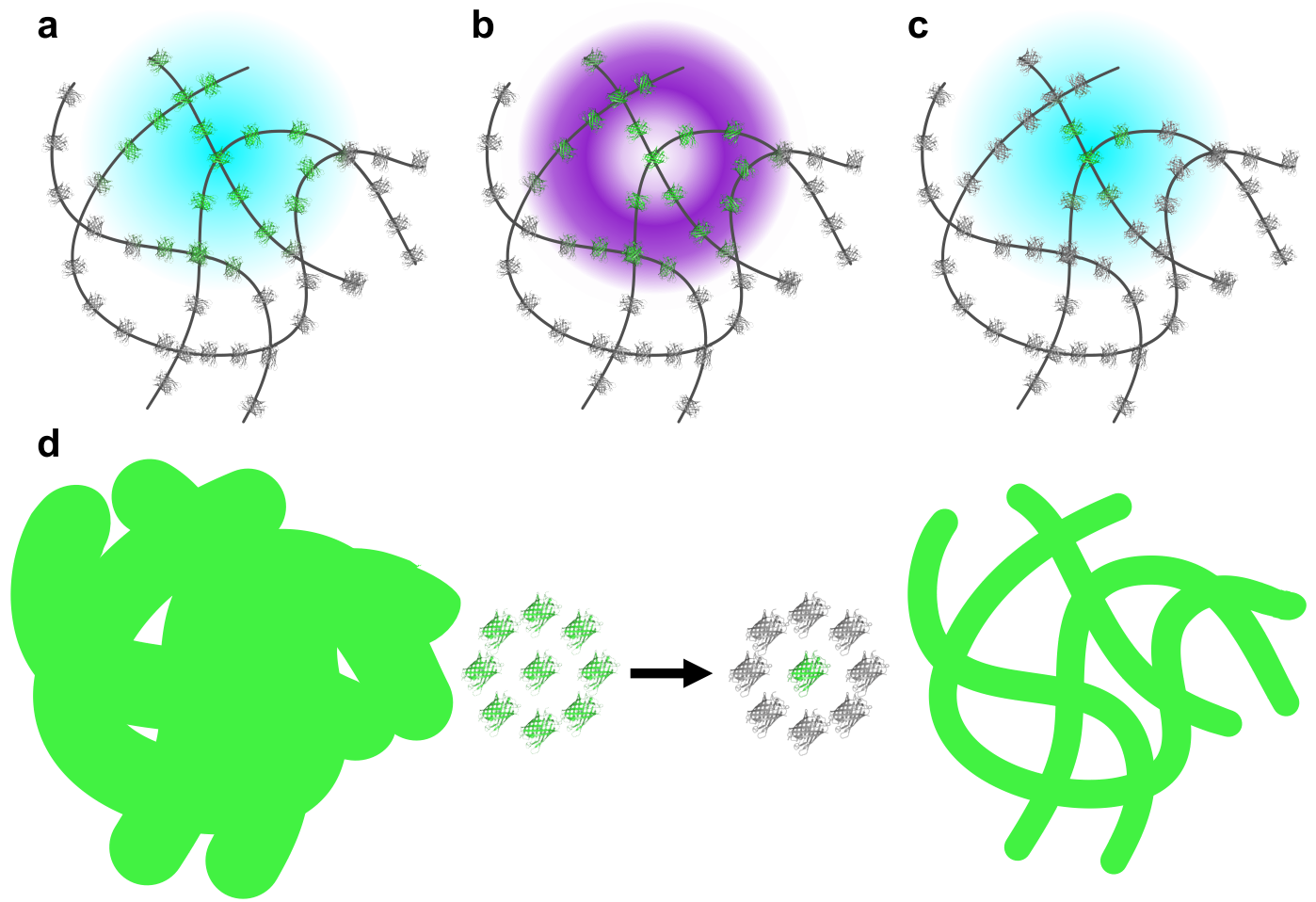

Figure 1.9: RESOLFT principle for a positively switching RSFP. (a-c) RESOLFT switching scheme. RSFPs are initially switched to the on-state with the activation wavelength (a). Subsequently, peripheral fluorophores are rendered non-fluorescent with a doughnut-shaped deactivation beam (b). The remaining central fluorophores in a subdiffraction volume are then read out with the same wavelength employed for activation (c). (d) Whereas diffraction-limited imaging results in indiscernible structures (left), RESOLFT nanoscopy restricts fluorescence emission to a nanoscale region (right).

While these first demonstrations of live-cell RESOLFT nanoscopy used light intensities of approximately $1 \mathrm{~kW} / \mathrm{cm}^{2}$ or less, pixel dwell times amounted to $20-40 \mathrm{~ms}$ [123, 144], which is unfavorable for imaging of live-cell dynamics. The imaging acquisition time was greatly improved by engineering or utilizing faster RSFPs for RESOLFT nanoscopy, namely rsEGFP2 [124] and Dronpa-M159T [126, 151], reaching pixel dwell times below $1 \mathrm{~ms}$. rsEGFP2 was also used for endogenous tagging of target structures [152] and was used for the first demonstration of RESOLFT nanoscopy in a living organism in Drosophila melanogaster [153]. Two-color RESOLFT nanoscopy could be facilitated by discerning two different RSFP labels either spectrally [154] or based on their fluorescence lifetime [5]. In 2019, smart RESOLFT was published as a method which accelerates point-scanning RESOLFT 


\section{Introduction}

nanoscopy by probing the fluorescence signal at every pixel before employing the switching sequence. This approach subsequently omits switching and accelerates the scanner in regions with a fluorescence signal below a defined threshold [155].

Alternatively, the parallelization of the RESOLFT concept was already proposed in the first publication with asFP595 [51] to accelerate image acquisition. By employing a deactivation pattern with multiple regions of zero intensity separated by a distance larger than the diffraction limit, the separation of states required for nanoscopy can be facilitated at a large number of positions at the same time. This proposal was facilitated with a grid of point-like zeros, where widefield illumination was used for activation and readout with a camera [156]. The concept could also be shown in a dual-color realization [157], and Wang et al. demonstrated consecutive imaging of 50 frames with a parallelized RESOLFT microscope [158]. Parallelized RESOLFT nanoscopy was later extended by employing multi-focal activation and readout instead of widefield illumination [129, 159]. Another approach utilized a pattern of line-shaped zero-intensity regions to combine SIM with the non-linearity introduced by the RSFP Skylan-NS [128], which was thus denoted as patterned activation non-linear SIM (PA NL SIM) [160].

However, all the implementations of RESOLFT nanoscopy described above, with the exception of the asFP595 proof of concept, utilized negatively switching RSFPs or Dreiklang with decoupled switching. While the former have a limited photon budget due to their deactivation during readout, Dreiklang requires rather long imaging dwell times in point-scanning approaches [144].

\subsubsection{Nanoscopy with positively switchable RSFPs}
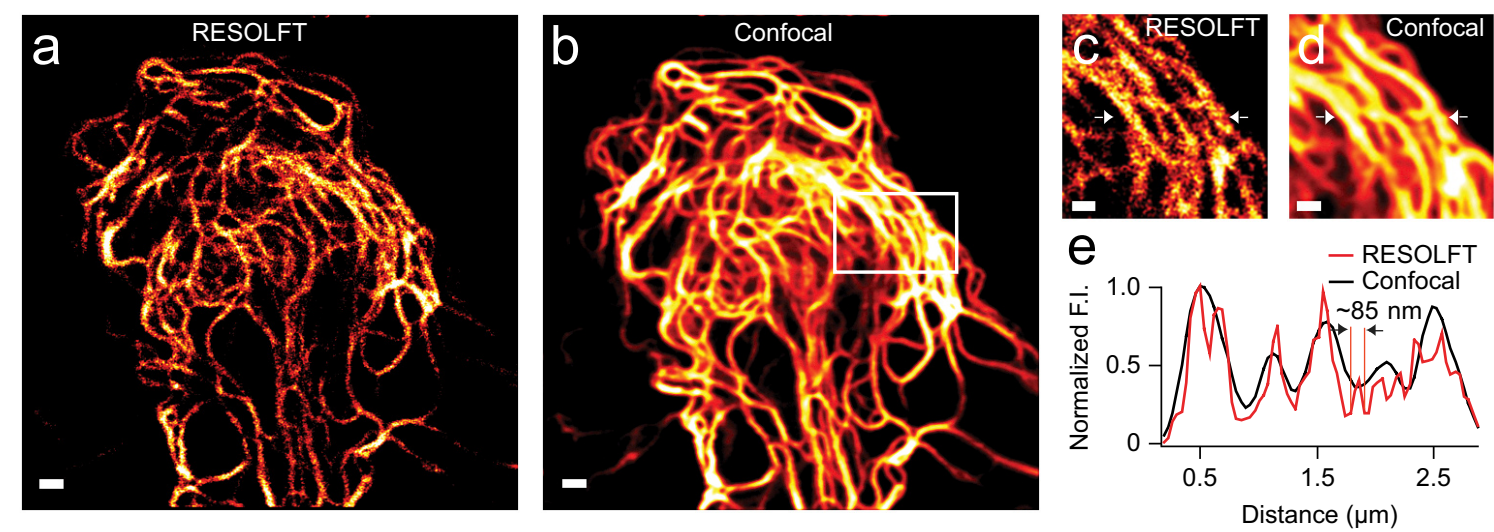

Figure 1.10: Point-scanning RESOLFT microscopy with Kohinoor. (a-d) HeLa cells expressing vimentin-Kohinoor imaged in RESOLFT (a,c) and confocal (b,d) mode. The boxed region in $\mathrm{b}$ marks the field of view in $\mathrm{c}$ and $\mathrm{d}$. (e) Line profiles at the positions indicated by arrows in c and d. $1 \mathrm{~ms}$ pixel dwell time with $400 \mu \mathrm{ss}$ readout duration were employed for RESOLFT imaging. Scale bars: $1 \mu \mathrm{m}(\mathrm{a}, \mathrm{b})$ and $500 \mathrm{~nm}(\mathrm{c}, \mathrm{d})$. Adapted by permission from Springer Nature Customer Service Centre GmbH: Springer nature, NATURE METHODS [121], () 2015. 
Positively switching RSFPs are expected to overcome the photon budget limitations of negatively switching RSFPs in RESOLFT nanoscopy by simultaneous employment of the doughnut-shaped off-switching laser beam with the Gaussian-shaped readout laser, thereby keeping RSFPs in the periphery in the non-fluorescent off-state. However, only few positively switching RSFPs have been published so far with even less nanoscopy applications [120-122, 161]. Padron was the first green-emitting positively switching RSFP with low residual offstate fluorescence intensity [120], but has not been used in nanoscopy applications.

a

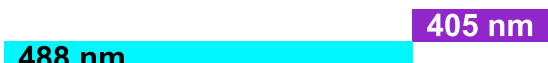

\section{$488 \mathrm{~nm}$}
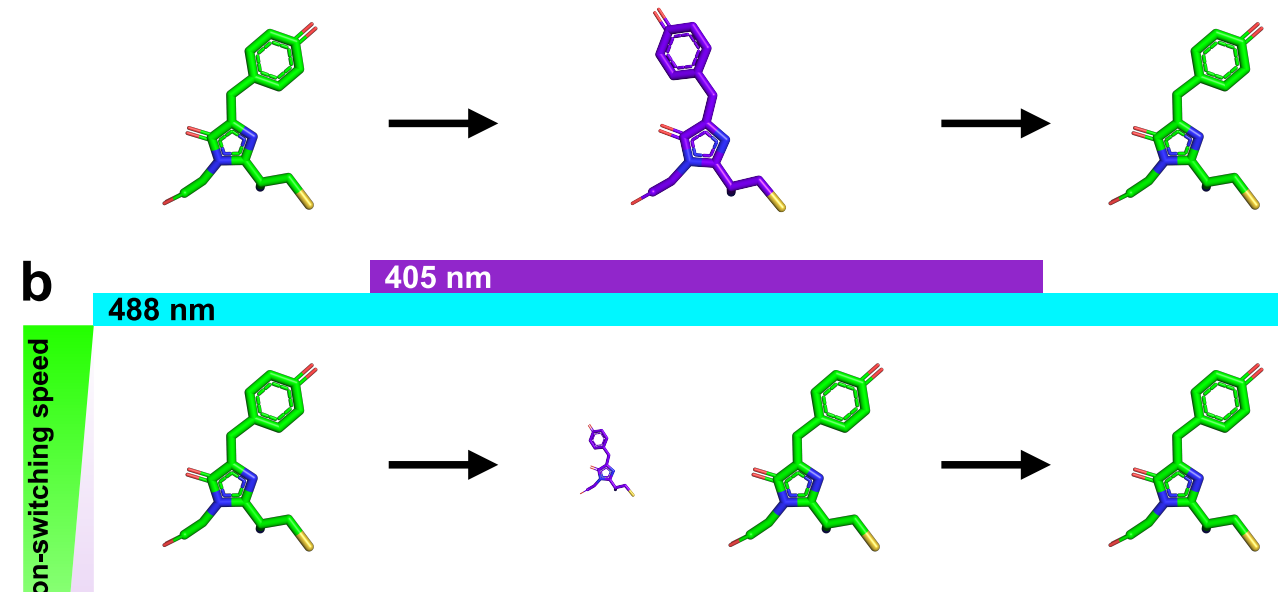

\section{8 nm}
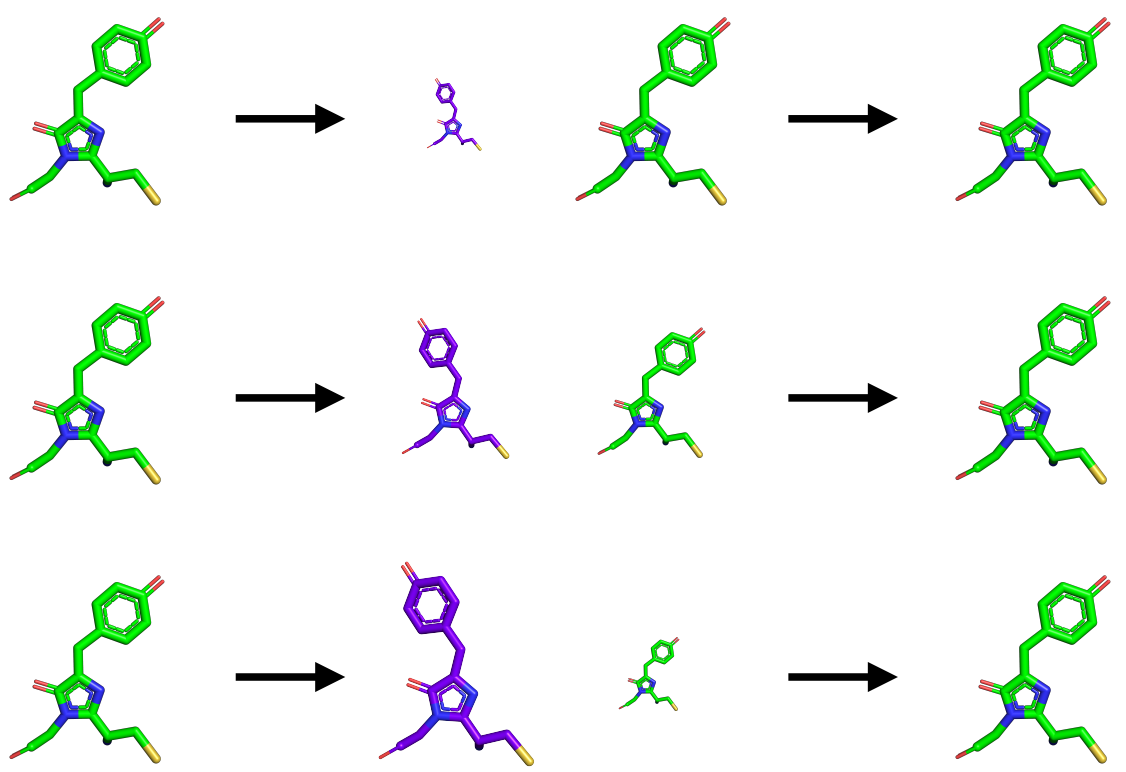

Figure 1.11: Influence of irradiation intensity on the switching state of Padron. (a) Sequential switching leads to almost complete reversible saturation of the on-state (green cis-chromophore) or off-state (purple trans-chromophore) of Padron. (b) If both on- and off-switching irradiation is applied simultaneously, the saturation of states depends on the relative light intensities as well as the rate constants for activation and deactivation.

Nevertheless, it was used to demonstrate diffraction-limited imaging of spectrally overlapping RSFPs in different channels [120, 162]. Another use was in studies on fluorescence detected sedimentation velocity $[163,164]$ or the switching capabilities of RSFPs at cryotemperatures [165].

Aside from asFP595 [51], the only positively switchable RSFP that has been used for super-resolution microscopy is Kohinoor, which is based on Padron and has been published 


\section{Introduction}

during the course of this thesis [121]. It has been used in both point-scanning RESOLFT nanoscopy [121] and non-linear SIM [161], a resulting RESOLFT image is shown in figure 1.10. However, while this image displays an effect in response to RESOLFT imaging, it lacks the increase of structural information that is typically achieved with established RSFPs in RESOLFT nanoscopy [124]. In addition, the off-switching kinetics of Kohinoor were improved only threefold in comparison with Padron. This is only a minor improvement and expected to complicate off-switching under simultaneous $488 \mathrm{~nm}$ irradiation. In order to prolong fluorescence readout of a positively switching RSFP in RESOLFT nanoscopy after the deactivation step, both the doughnut-shaped deactivation and the Gaussian-shaped readout laser have to be applied simultaneously. The applied intensities have to result in a sufficient separation of saturable states, that is, switching peripheral RSFPs to the off-state while central ones remain fluorescent. This concept requires fast off-switching rates compared to the rate of activation at a given combination of intensities (fig. 1.11). 


\section{Aim of thesis}

The aim of this thesis was to develop a positively switchable RSFP with emission in the green VIS range with switching characteristics that allow to utilize the conceptual benefits of the positive switching regime for RESOLFT nanoscopy. For this purpose, Padron [120] was chosen as a template. While Padron was reported to be switchable to a very low residual offstate fluorescence intensity below $1 \%$, its inefficient expression and maturation at $37^{\circ} \mathrm{C}$, low switching fatigue, and slow switching rendered it unsuitable for live-cell RESOLFT nanoscopy. Consequently, no super-resolution images have been published with Padron as a label.

The presented thesis describes the mutagenesis strategy employed to improve Padron and the subsequent characterization in comparison with Kohinoor, which was generated from Padron as well [121]. The implementation of live-cell point-scanning RESOLFT nanoscopy is shown to demonstrate the improved performance of the novel variant. 


\section{Results}

In this study, the RSFP Padron [120] was improved by performing multiple rounds of mutagenesis, screening, and selection of improved variants. The following sections will report on the results of mutagenesis and summarize its important stages. The resulting improved variants will then be characterized in detail in comparison to Padron and Kohinoor, while the variant deemed most applicable for RESOLFT nanoscopy will be used for the demonstration of super-resolution microscopy with a positively switching RSFP. Before that, the following section will describe the improvements of the automated screening microscope used for screenings after mutagenesis in order to increase sample throughput.

\subsection{Improvement of the automated screening microscope}

Padron was improved in a process of repeated mutagenesis. In each step, the template sequence was altered either at a single base triplet or randomized across the whole sequence. This resulted in a large number of mutated variants, which were screened for improved switching characteristics. The majority of measurements associated with this process were performed in bacterial colonies on agar plates at a fluorescence microscope capable of automated colony sampling (fig. 3.1a). Time-resolved fluorescence was recorded in response to a predefined laser sequence which induced switching of Padron variants (cf. methods sec. 5.3). However, the number of variants that could be screened in each round was limited by the operation time of the automated screening microscope. To increase screening capacities and to accelerate the throughput, colony sampling and focusing were improved by enhancing the control software of the automated screening microscope.

\subsubsection{Improving speed and precision of colony sampling}

In the original state of the automated screening microscope, agar plates were sampled by means of a rigid grid (fig. 3.1b). Range and step size of the grid were defined by the user prior to a screen, which resulted in various impediments. Independent from the colony density on an agar plate, all coordinates set by the grid had to be probed, causing repetitive acceleration and stopping of the stage even in blank areas of an agar plate. The software allowed for probing of fluorescence at every grid coordinate before an autofocus and a measurement were run to reduce screening duration. However, using this option, the user had to compromise between speed and sensitivity as probing could occur out of focus resulting in false negatives. Lowering the threshold for probing on the other hand increased the number of false positive measurements.

Grid-based sampling also had implications for colony selectivity. When a coarse grid was chosen, colonies were more likely to either lie outside of the grid and be missed during a screen or to be measured at their outer perimeter, resulting in aberrant fluorescence intensity 
a
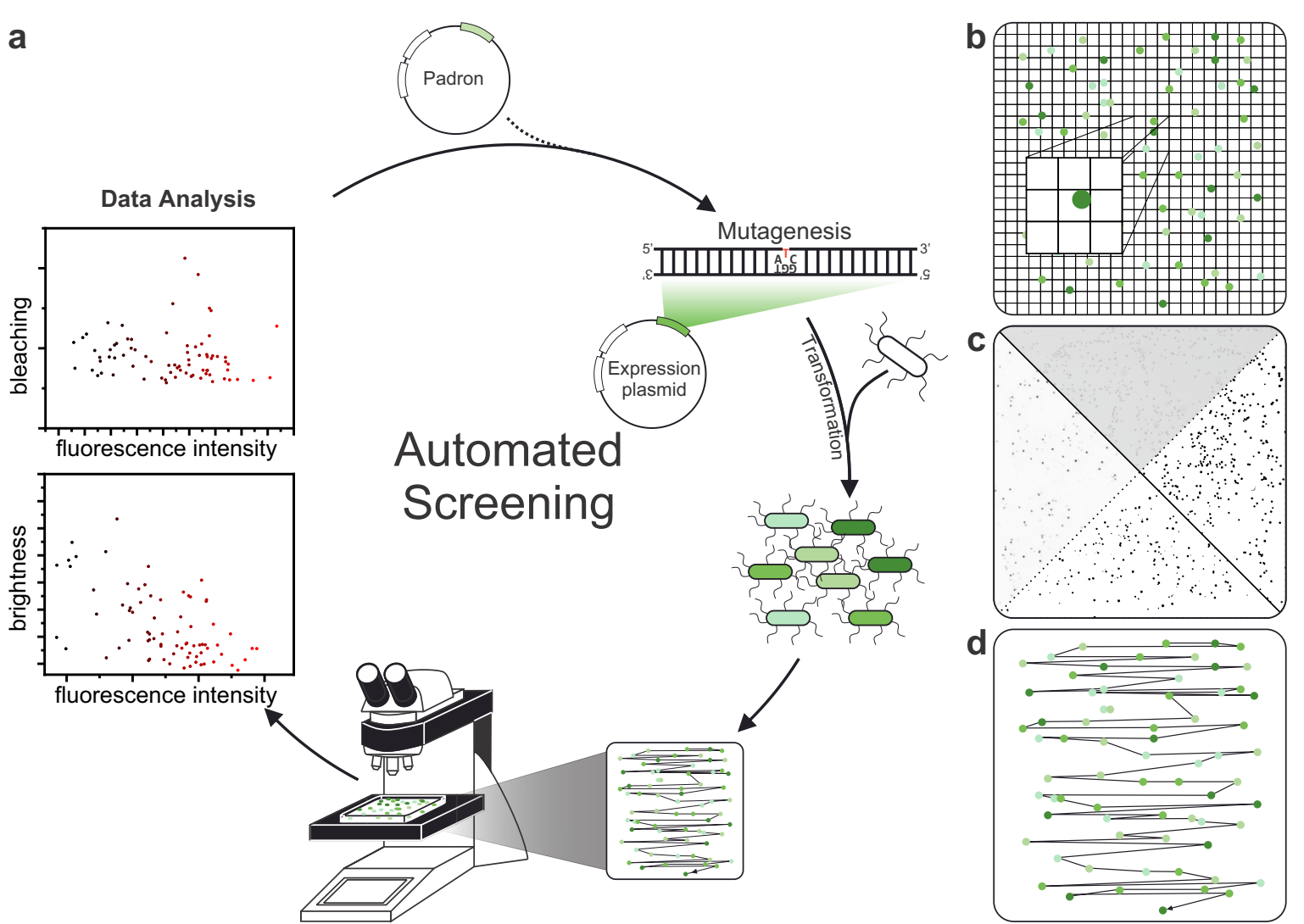

Figure 3.1: Automated bacterial screening with colony selectivity. a) Workflow of a screening round. b) Original sampling of agar plates before improvement. Plates were screened by means of a fixed grid with predetermined step size. c) Plate processing for the improved sampling. Colony coordinates were determined with images based either on fluorescence intensity (left and bottom quadrant) or via colony light scattering during trans illumination (top and right quadrant) of the agar plates. Coordinates were calculated after thresholding. d) Improved sampling of agar plates. All colonies were targeted directly and centered.

measurements. Conversely, when the grid was too dense the probability of repeated measurements of the same colony became higher, increasing both screening time and data noise.

To address these drawbacks, colony sampling was improved. In detail, agar plates were first digitized with the Amersham Imager 600RGB (GE Healthcare, Chicago, IL, USA) based either on fluorescence intensity or on light scattering of colonies during trans illumination. Recorded plate images were thresholded in the Fiji distribution of imageJ (v1.52p, [166, 167]) (fig. 3.1c), followed by calculation of colony pixel coordinates in the image based on center of weight and circularity. To transform pixel coordinates into stage coordinates of the microscope, three or more colonies were chosen for calibration. All calculations were made available to users of the screening setup with an imageJ macro, and a new function programmed with LabVIEW (National Instruments, Austin, TX, USA) was added to the software controlling the microscope.

These improvements increased throughput of bacterial screenings: With good calibration every colony was measured only once in its center (fig. 3.1d). Due to the necessity of digitizing 


\section{Results}

the plate layout before screenings, colony selectivity was introduced as a powerful new tool: Colonies could be measured selectively based on on their fluorescence intensity by excluding weakly fluorescent clones during thresholding of plate images. As circularity of thresholded colonies was considered for calculation of pixel coordinates, overlapping colonies, which would result in a plasmid mixture of mutants after colony picking, could be excluded from screenings. This allowed for screening of densely populated agar plates that previously would have been discarded because data analysis and clone picking would have been considered as being too tedious. With the improved sampling isolated colonies could easily be measured selectively. This was also useful for the detailed characterization of Padron variants as it allowed for a larger number of colonies and thus more mutated variants to be measured with equal precision.

\subsubsection{Accelerating the autofocus}

After the colony sampling had been improved, the autofocus (AF) of the automated screening setup was the most time consuming factor aside from the actual measurements. The original autofocus was precise but slow. The stage was incrementally moved along the optical axis for focusing, and fluorescence intensity was probed at each position. Data points were then interpolated with spline interpolation. The separation of stage movement and probing limited the focal speed. To address this, a new autofocus function was developed for the LabVIEW control software. Focal speed was accelerated by continuously moving the stage and simultaneously probing fluorescence with a single, long measurement. This resulted in time-dependent peaking of fluorescence; the peak position on the time axis was representative of the focal position within the bacterial colony. To correlate the time axis of this measurement with the position along the optical axis, a calibration had to be performed. For this purpose, 9 colonies were measured with both the old and the new autofocus. By performing a linear regression of spatial and time-dependent fluorescence peak positions from both measurements, the focal position could be calculated from data obtained with the new autofocus mode.

To compare the new autofocus mode with the old one, bacterial colonies expressing mNeonGreen [168] were focused with both the original and the improved autofocus, and the fluorescence was subsequently probed at the resulting z-coordinates (cf. methods sec. 5.3.1.2). Peak fluorescence intensities differed by less than $5 \%$ for most colonies, and focal differences of both modes were mostly smaller than $10 \mu \mathrm{m}$ (fig. 3.2a, b). This focal difference was uncritical, and observed differences of fluorescence intensities were smaller than the ones resulting from other factors such as laser intensity fluctuations, colony inhomogeneity, or day-to-day variation of protein expression (fig. 3.2a, d). The new autofocus could reduce duration of a single autofocus measurement by approximately $85 \%$ from $22.1 \pm 0.68 \mathrm{~s}$ to $3.3 \pm 0.03 \mathrm{~s}$ (fig. $3.2 \mathrm{c}$ ) for the given autofocus range. Coarser sampling would have accelerated the old autofocus was well, but in a time frame of $\sim 3$ seconds only 5 -10 sampling steps could have been performed, which would have been insufficient for precise focusing and simultaneously covering 

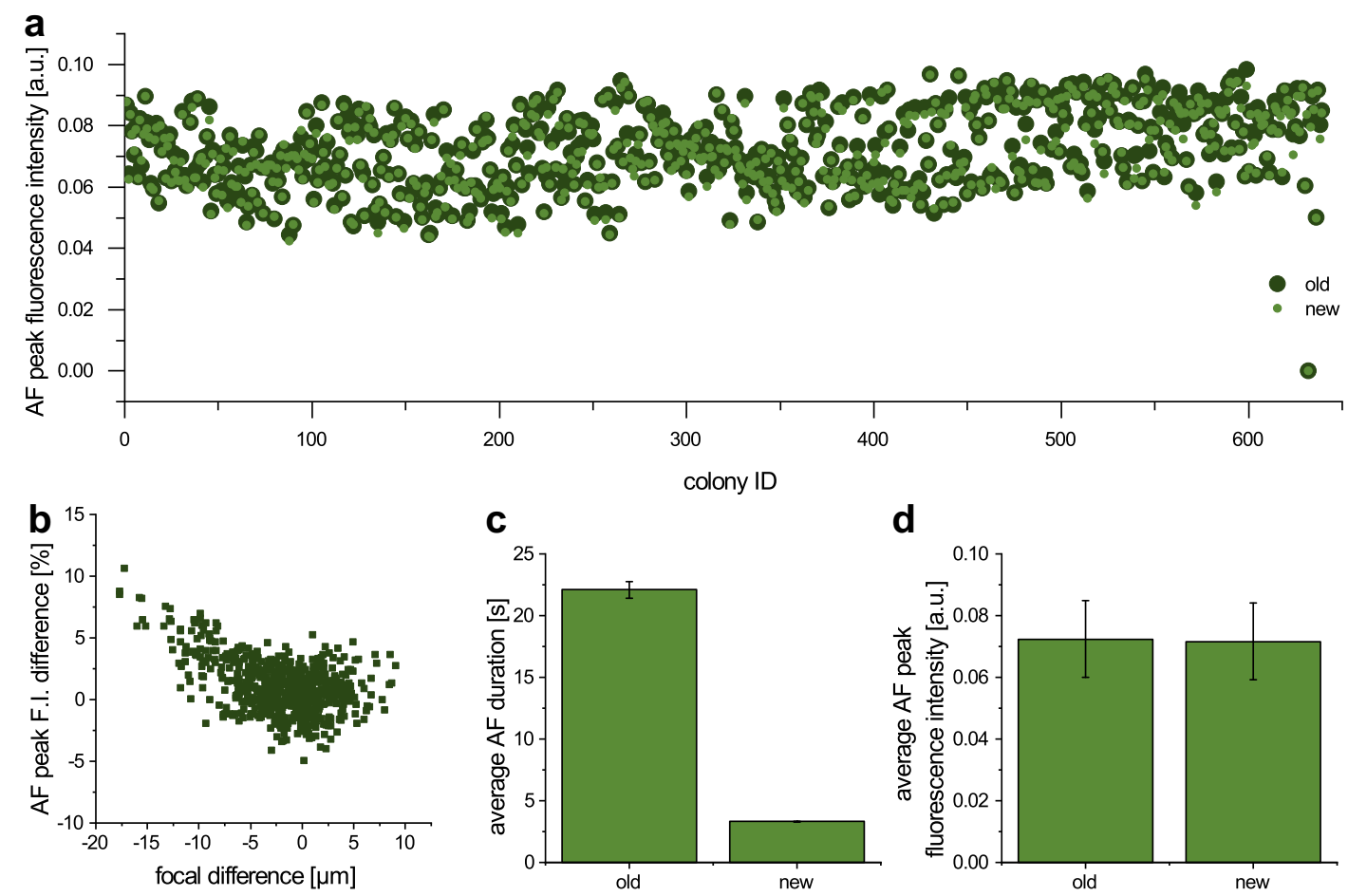

Figure 3.2: Improved autofocus for bacterial screening. Old and new autofocus modes were compared in bacterial colonies expressing mNeonGreen [168]. a) Probed fluorescence intensity (F.I.) at the peak z-coordinates determined with the old and new autofocus mode. b) Relation between fluorescence intensity differences as percentage of the F.I. at the old AF position and the focal difference of both AF modes. c) Average AF duration. d) Averaged probed fluorescence intensities. 630 colonies were measured on a single agar plate.

a large focal range. The improvement thus accelerated screenings and increased the number of colonies that could be screened per mutagenesis and per day.

While the improved autofocus and was developed towards the end of this study, the improved colony sampling could increase the throughput of later screenings, including the generation of Padron 2.0. Padron 2.1, which was the last variant generated in the course of this thesis. It emerged from a directed evolution approach (cf. methods sec. 5.3.1) with multiple rounds of screenings which benefited from both improvements. The following section will give an overview over the course of mutagenesis of Padron, which resulted in three new Padron variants, namely Padron 1.9, 2.0 and 2.1.

\subsection{Padron mutagenesis}

In order to improve Padron, is was subjected to repeated rounds of mutagenesis and screening with selection and characterization of candidate variants at the automated screening microscope. For this purpose, time-resolved fluorescence of bacterial colonies of E. coli transformed with the mutated expression plasmids was recorded (cf. methods secs. 5.2 and 5.3). The switching was performed by alternating irradiation with $405 \mathrm{~nm}$ and $488 \mathrm{~nm}$ lasers, giving insight into switching capabilities of Padron mutants. By adapting measurement parameters 


\section{Results}

between rounds of mutagenesis this method revealed Padron variants with improved switching characteristics.

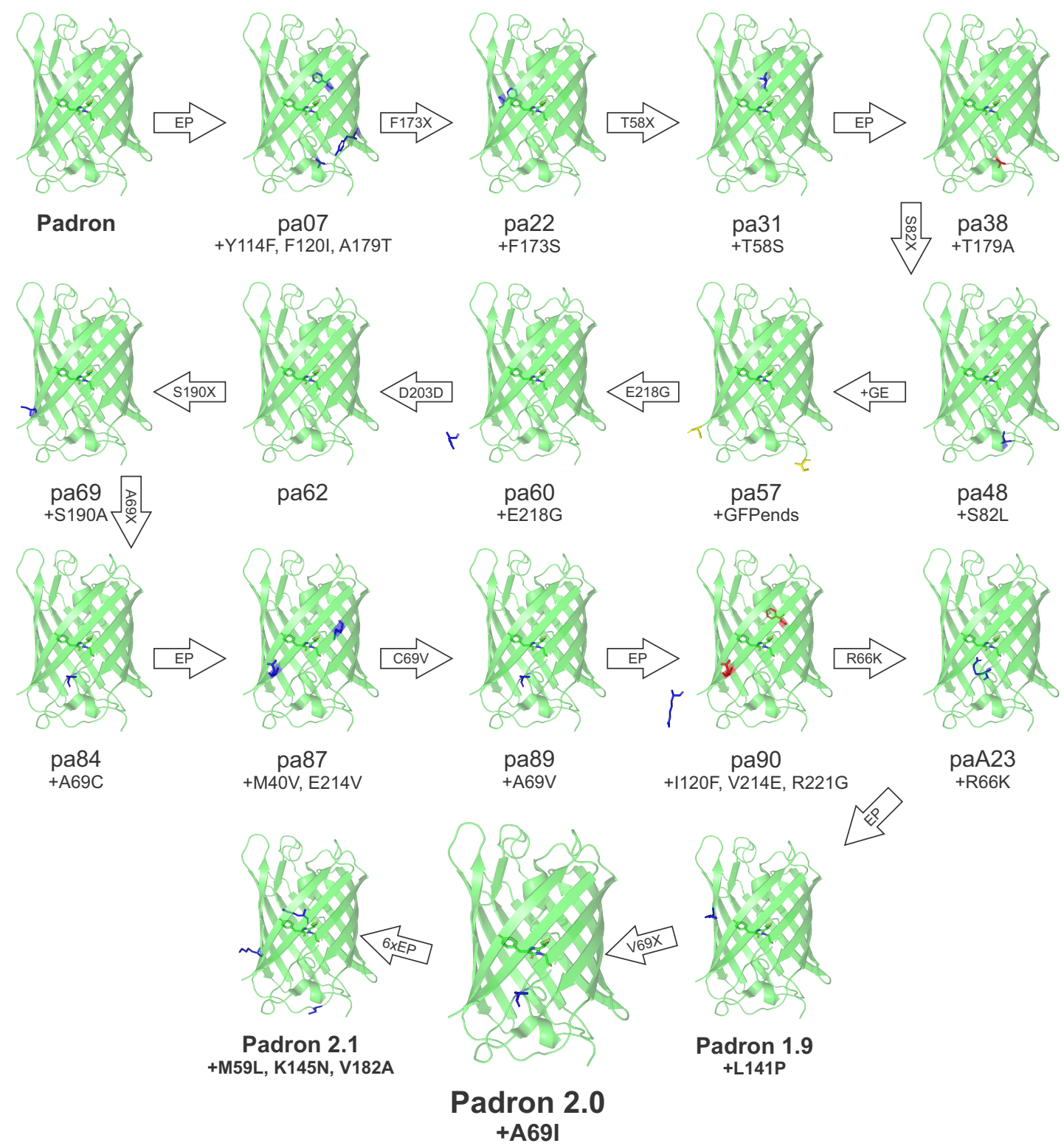

Figure 3.3: Padron mutagenesis. Protein structures displayed are based on the fluorescent on-state of Padron (PDB ID: 3ZUJ). Padron variants are labeled according to internal IDs assigned during mutagenesis and new mutations of the respective variant are displayed below the ID. Mutated positions for each variant are highlighted in stick representation with blue representing new mutations and red marking reversions to the amino acids originally present at the given position in Padron. Yellow amino acids mark the $\mathrm{N}$ - and C-terminal amino acids in the structure where GFPends were attached. Pa60 and pa90 representations have amino acids situated outside of the barrel as positions 218 and 221 are not part of the solved crystal structure. Arrows are labeled with the mutagenesis protocol performed in each step. EP, error-prone mutagenesis; +GE, attachment of GFPends. Amino acids are numbered based on Dronpa. 
Padron was mutated with a combined approach of randomized error-prone mutageneses and site-directed mutageneses (SDMs). The latter were either based on positions that were known to have an effect on switching dynamics or protein characteristics of Padron, Dronpa, or related proteins, such as Phe173 [131, 169], Ala69 [169], and Glu218 [142], or on positions that emerged during error-prone mutagenesis. This approach resulted in Padron 2.0 as an improved version of Padron with 11 mutations (M40V, T58S, R66K, A69I, S82L, Y114F, L141P, F173S, S190A, E218G, R221G) and altered N- and C-termini (N-terminus: +MVSKGEENNMA, C-terminus: +MDELYK), a more detailed description of critical steps in the mutagenesis process will be given in the following section. Figure 3.3 depicts the detailed progress of mutagenesis and shows the order in which mutations were introduced. Variants up to and including pa69 were created by Dr. Tim Grotjohann in our group. Padron 1.9, the direct precursor of Padron 2.0, and Padron 2.1 (Padron 2.0 +M59L, K145N, V182A), the result of directed evolution of Padron 2.0, are notable variants with distinct characteristics. These variants, hereafter in part referred to as new or final variants, will be analyzed and discussed below along with Kohinoor, a variant based on Padron that was published in 2015 by Tiwari et al. [121]. A sequence alignment of Padron, Kohinoor and the improved variants presented in this thesis is shown in figure 3.4.

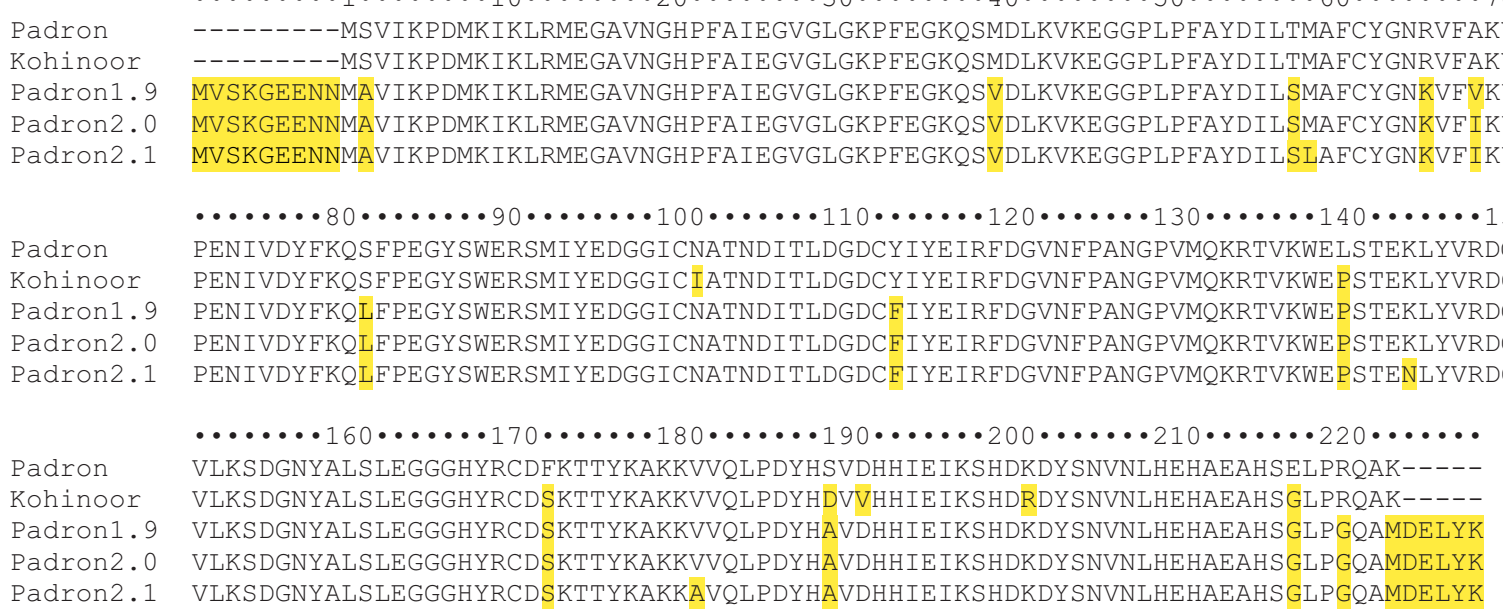

Figure 3.4: Protein sequence alignment of new Padron variants and Kohinoor to Padron. Amino acids differing from Padron are marked in yellow. Numbering of amino acids is based on Padron, which is in accordance with numbering in Dronpa.

\subsection{Switching parameters improvement}

Because the intermediate variants, which were generated from Padron before Padron 1.9, 2.0 and 2.1 were obtained, had switching characteristics that were unsuitable for RESOLFT nanoscopy, they were not characterized in detail in this thesis. However, in order to elucidate the influence of single mutations on switching characteristics they were subjected to a com- 


\section{Results}

parative measurement at the automated screening microscope. More specifically, proteins were expressed in E. coli and measured with irradiation doses of $405 \mathrm{~nm}$ light for off- and $488 \mathrm{~nm}$ light for on-switching, which were not changed between measurements. The doses were adjusted to the switching performance of Padron 2.0 and were therefore not adapted to the performance of intermediate variants, which had implications for the interpretation of data. The measured residual off-state fluorescence intensity of a slow protein for example did not allow for a statement regarding its absolute ensemble switching background because of incomplete switching. Nevertheless, this method constituted a good estimate of key differences between proteins as well as variant-to-variant improvements (cf. methods sec. 5.3.1.4). It was also similar to the comparative characterization of candidate variants performed between mutageneses, where mutants were analyzed with fixed measurement parameters.

The automated screening microscope allowed for parallel and versatile estimation of protein characteristics based on fluorescence intensity measurements in bacterial colonies. By probing time resolved fluorescence intensity during irradiation the following protein characteristics were determined:

- Fluorescence intensity as the maximal ensemble fluorescence intensity in the onstate.

- Switching half-time from off- to on-state as half-time needed to switch from the residual fluorescence after $405 \mathrm{~nm}$ irradiation to the maximal fluorescence intensity in the on-state.

- Residual off-state fluorescence intensity as fluorescence intensity after $405 \mathrm{~nm}$ irradiation relative to the maximal on-state fluorescence intensity.

- Residual off-state fluorescence intensity with simultaneous $488 \mathrm{~nm}$ illumination as fluorescence intensity after concurrent 405 and $488 \mathrm{~nm}$ irradiation relative to the saturated on-state fluorescence intensity.

- Relative on-state fluorescence intensity after 250 switching cycles as maximal on-state fluorescence intensity after cycling between on- and off-state 250 times relative to to the saturated on-state fluorescence intensity of the first cycle (switching fatigue).

- Maximum/end fluorescence intensity as the ratio of the maximal fluorescence intensity of the activation curve and the saturated on-state fluorescence intensity. Some intermediate variants displayed activation curves with a bright intermediate state, which was quantified with this ratio.

This section will focus on the progress of mutagenesis and will highlight the key mutations introduced that led to important improvements to Padron. A detailed comparison of Padron and final variants will be shown in section 3.4. The results presented in this part represent solely the switching performance in bacterial colonies, other characteristics such as oligomerization tendency or tagging performance of cellular structures were not analyzed for intermediate variants. The reasoning behind this was that variants with insufficient switching performance in E. coli were unsuitable for live-cell applications. 


\subsubsection{Switching characteristics of intermediate variants}
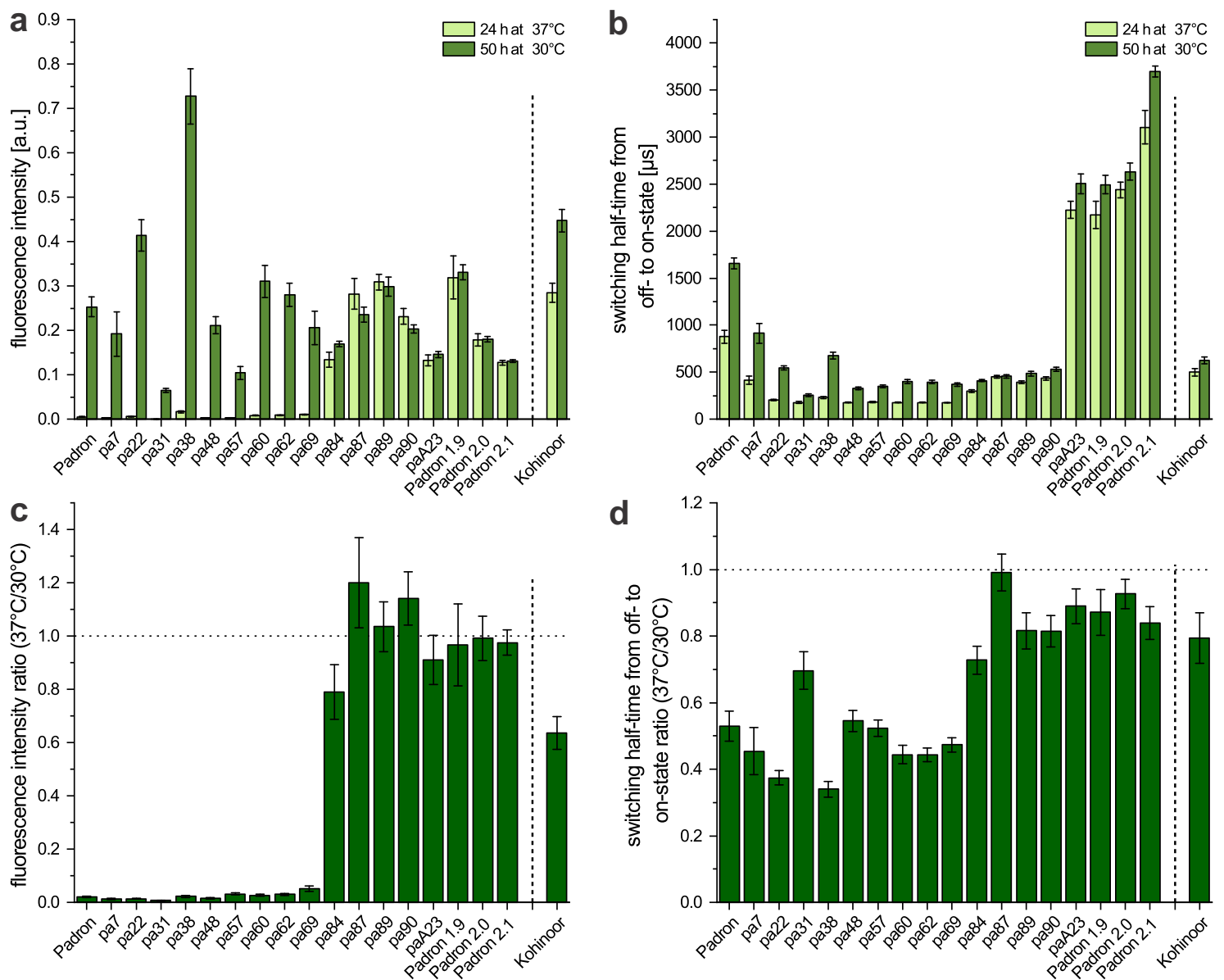

Figure 3.5: Fluorescence intensity and switching half-time of intermediate Padron variants. (a) Fluorescence intensities measured after different growth conditions with (c) respective ratios of $37^{\circ} \mathrm{C}$ and $30^{\circ} \mathrm{C}$ data. (b) Switching half-times measured after different growth conditions with (d) respective ratios of $37^{\circ} \mathrm{C}$ and $30^{\circ} \mathrm{C}$ data. E. coli colonies were measured after growth at $37^{\circ} \mathrm{C}$ for $24 \mathrm{~h}$ or at $30^{\circ} \mathrm{C}$ for $50 \mathrm{~h}$. Data represents averaged measurements of 10 colonies.

The original Padron displayed poor expression at $37^{\circ} \mathrm{C}$. While the expression was not impaired at $30^{\circ} \mathrm{C}$, this drawback had implications for live-cell applications as most experiments are performed at $37^{\circ} \mathrm{C}$. One focus of the mutagenesis process was thus to improve expression at this temperature. In order to reveal this improvement, the comparative measurements of all Padron variants generated in this thesis were performed after two different growth conditions: $50 \mathrm{~h}$ at $30^{\circ} \mathrm{C}$ for variants with slow maturation and impaired expression, and $24 \mathrm{~h}$ at $37^{\circ} \mathrm{C}$ to reveal improved maturation and expression. In these measurements, Padron 2.0 and 2.1 displayed lower fluorescence intensity than the original Padron after full maturation (fig. 3.5a). Although some variants such as pa38 were brightly fluorescent in bacterial colonies, this improvement was later lost in favor of other characteristics. Measurement of fluorescence intensity after different growth conditions revealed the impaired expression at $37^{\circ} \mathrm{C}$ of variants preceding pa84: Variants matured well after growth for $50 \mathrm{~h}$ 


\section{Results}

at $30^{\circ} \mathrm{C}$ but failed to give the same results after expression at $37^{\circ} \mathrm{C}$ for $24 \mathrm{~h}$. This became especially obvious when the ratio of fluorescence intensities measured at both conditions was calculated (fig. 3.5c). For all variants up to and including pa69, fluorescence intensity after maturation at $37^{\circ} \mathrm{C}$ was less than $10 \%$ of the value measured after maturation at $30^{\circ} \mathrm{C}$. To address this issue, position 69 was mutated via SDM because A69V had been reported to improve protein expression of mEosFP at $37^{\circ} \mathrm{C}[169] . \quad \mathrm{A} 69 \mathrm{C}$ in pa84 had the anticipated effect and all subsequent variants showed good expression at $37^{\circ} \mathrm{C}$ with similar absolute intensity values at $30^{\circ} \mathrm{C}$ expression. This effect was also observable for other characteristics, although it was less pronounced (figs. 3.5d, 3.6c, d). However, A69C caused aberrant switching behavior after multiple cycles such as inconsistent kinetics and therefore continuously increasing residual off-state fluorescence intensity (data not shown). Ala69 was therefore replaced with valine in pa89, which resulted in similar expression improvement but caused less problems with the consistency of characteristics compared to pa87.
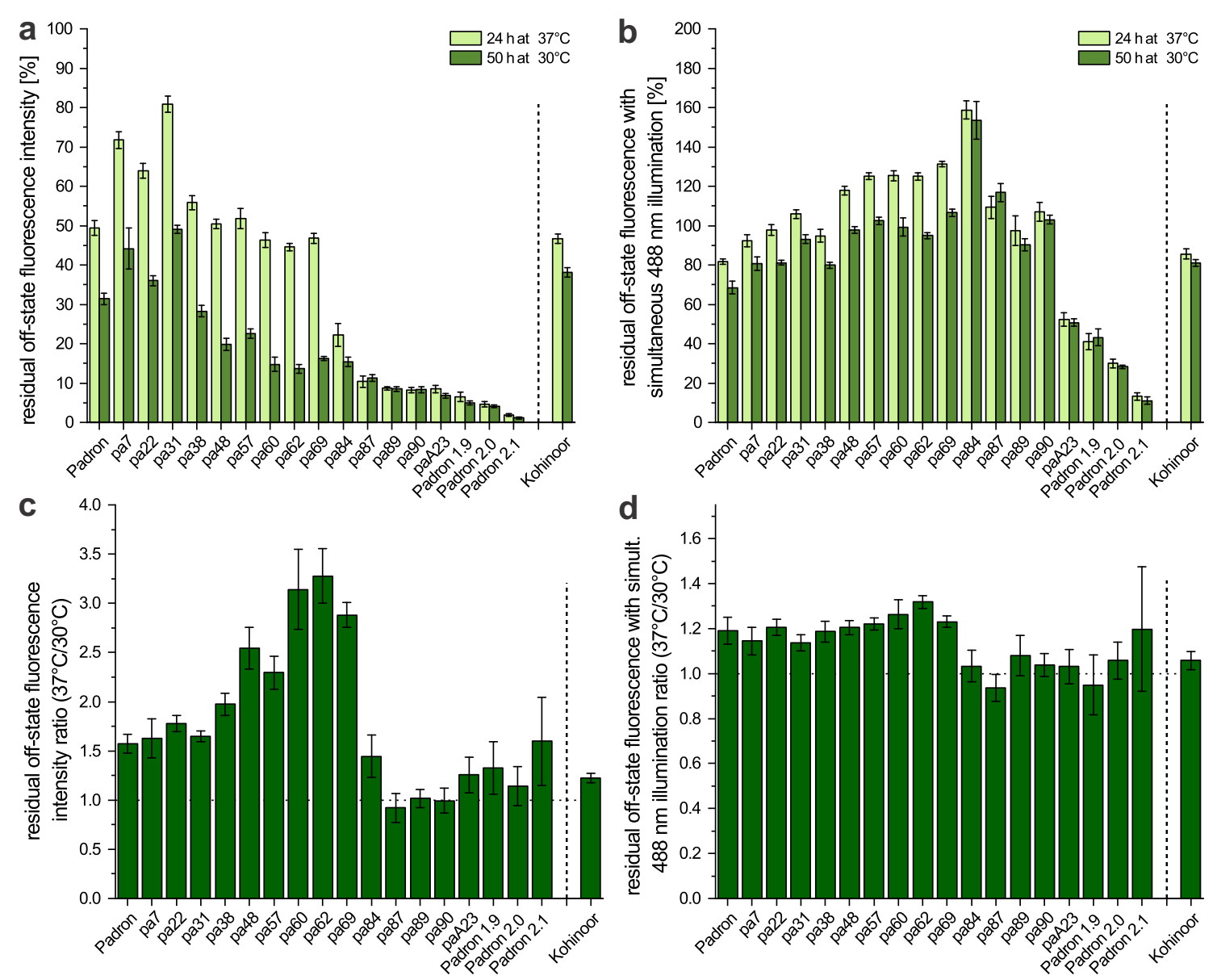

Figure 3.6: Residual off-state fluorescence intensity of intermediate Padron variants. (a) Residual off-state fluorescence intensities after $405 \mathrm{~nm}$ irradiation at different growth conditions with (c) respective ratios of $37^{\circ} \mathrm{C}$ and $30^{\circ} \mathrm{C}$ data. (b) Residual off-state fluorescence intensities after 405 and $488 \mathrm{~nm}$ irradiation at different growth conditions with (d) respective ratios of $37^{\circ} \mathrm{C}$ and $30^{\circ} \mathrm{C}$ data. E. coli colonies were measured after growth at $37^{\circ} \mathrm{C}$ for $24 \mathrm{~h}$ or at $30^{\circ} \mathrm{C}$ for $50 \mathrm{~h}$. Data represents averaged measurements of 10 colonies. 
Because at this point maturation and expression problems had been solved, further mutagenesis focused predominantly on increasing the efficiency of ensemble switching to the off-state as well as improving switching fatigue. These two characteristics were impaired in the variants generated so far but are essential for RSFP performance in RESOLFT nanoscopy. Although absolute residual off-state fluorescence intensity could not be measured with the characterization method used during and between screenings due to incomplete switching, residual off-state fluorescence after a given $405 \mathrm{~nm}$ irradiation dose allowed for a good estimate of switching efficiency. This characteristic was continuously improved in variants following pa84 (fig. 3.6a) but was associated with much slower switching half-times from off- to onstate (fig. 3.5b). However, a detailed comparison of switching half-times was not possible based on the data shown here because return to the on-state did not occur from an saturated ensemble off-state but rather after incomplete off-switching, and curve shapes of the activation differed between variants. For mutants with similar residual off-state fluorescence intensities the measured half-times could be used to estimate switching speeds.

The key mutation for more efficient off-switching was R66K, which was first found in a discarded variant during the error-prone mutagenesis that resulted in pa90. This discarded variant displayed extraordinary performance with regards to switching fatigue, but fluorescence intensity measured was very low. R66K was thus introduced to pa90 and improved off-switching efficiency while lowering fluorescence intensity within an acceptable range for the following variant paA23 (figs. 3.6a, 3.5a). Interestingly, switching fatigue initially was impaired but could be rescued after a repeated site-directed mutagenesis at position 69 that exchanged valine for isoleucine in Padron 2.0 (fig. 3.7).

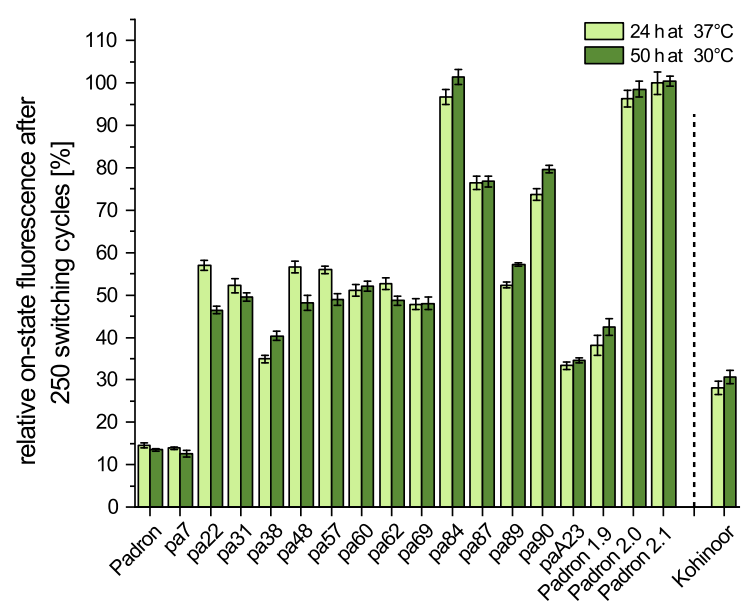

Figure 3.7: Relative on-state fluorescence intensity after 250 switching cycles of intermediate Padron variants. E. coli colonies were measured after growth at $37^{\circ} \mathrm{C}$ for $24 \mathrm{~h}$ or at $30^{\circ} \mathrm{C}$ for $50 \mathrm{~h}$. Data represents averaged measurements of 10 colonies.

More important with regards to R66K was the improvement of the ensemble switching to the non-fluorescent off-state under simultaneous $488 \mathrm{~nm}$ illumination (fig. 3.6b). This value was expected to be a good measure for how well the return of RSFPs to the on-state could be suppressed during fluorescence readout in RESOLFT microscopy as it can be interpreted as 


\section{Results}

a measure of the kinetic ratios for off- and on-switching. For Padron variants before paA23, suppression of the on-state was nearly non existent at the given parameters, but simultaneous illumination rather resulted in residual off-state fluorescence intensity values above $100 \%$. This was because the residual fluorescence was calculated by dividing the first data point of the on-switching curve, which represented the off-state, by the last one, which represented the on-state. Variants with a value above $100 \%$ therefore displayed increased fluorescence intensities after simultaneous illumination rather than switching to a non-fluorescent off-state. This was due to a property of early Padron variants which displayed a bright intermediate state during activation.

\subsubsection{Bright intermediate state during activation of intermediate variants}

The bright intermediate state is a distinct characteristic of some Padron variants that prevented their usability for RESOLFT microscopy. This term describes a state with increased fluorescence intensity reached by intermediate variants during switching from the off- to the on-state, which displayed higher fluorescence than the equilibrated state. It was visible as an additional peak in the on-switching curve and was absent in the original Padron and the final variants (fig. 3.8a, b, c). To quantify this phenomenon, the ratios of the maximum and end values of on-switching curves were calculated. Repeated empirical observations in preliminary tests had shown that this ratio appeared to be dependent on the $488 \mathrm{~nm}$ intensity and that its decay was independent from irradiation (data not shown). It also depended on growth conditions and was only visible after $24 \mathrm{~h}$ at $37^{\circ} \mathrm{C}$ for some variants (fig. $3.8 \mathrm{~d}, \mathrm{f}$ ). Interestingly, it disappeared for all variants after some switching cycles, displaying a distinct switching fatigue (fig. 3.8e, g). PaA23 was the first variant where the bright intermediate state could not be found, suggesting R66K eliminated this feature. The existence of this behavior had severe implications for the applicability of early intermediate variants in RESOLFT microscopy. It emerged during screenings by selecting variants with a shorter half-time for on-switching, supposedly increasing imaging speed of RESOLFT microscopy. However, the response of these variants to $488 \mathrm{~nm}$ illumination was extremely dominant compared to the $405 \mathrm{~nm}$ response so that ensemble suppression of their return to the on-state during readout was impossible at the laser intensities employed. In fact, images recorded with both wavelengths simultaneously had even more counts than images recorded with $488 \mathrm{~nm}$ only, and this gain was larger than what could have been attributed to $405 \mathrm{~nm}$ induced autofluorescence of the cell (data not shown). Hence, the additional photons emitted from the bright intermediate state could compensate the loss of photons caused by switching to the off-state. This was also the reason for calculated residual off-state values above $100 \%$ measured with simultaneous illumination (fig. 3.6b). In these measurements, simultaneous irradiation with 405 and $488 \mathrm{~nm}$ induced fast cycling of fluorophores between the on- and off-state effectively pumping a fraction of proteins into the bright intermediate state. The fluorescence decay visible during the $488 \mathrm{~nm}$ on-switching step was thus presumably the fluorescence decay of Padron variants from this state of increased fluorescence intensity to the equilibrated on-state. 

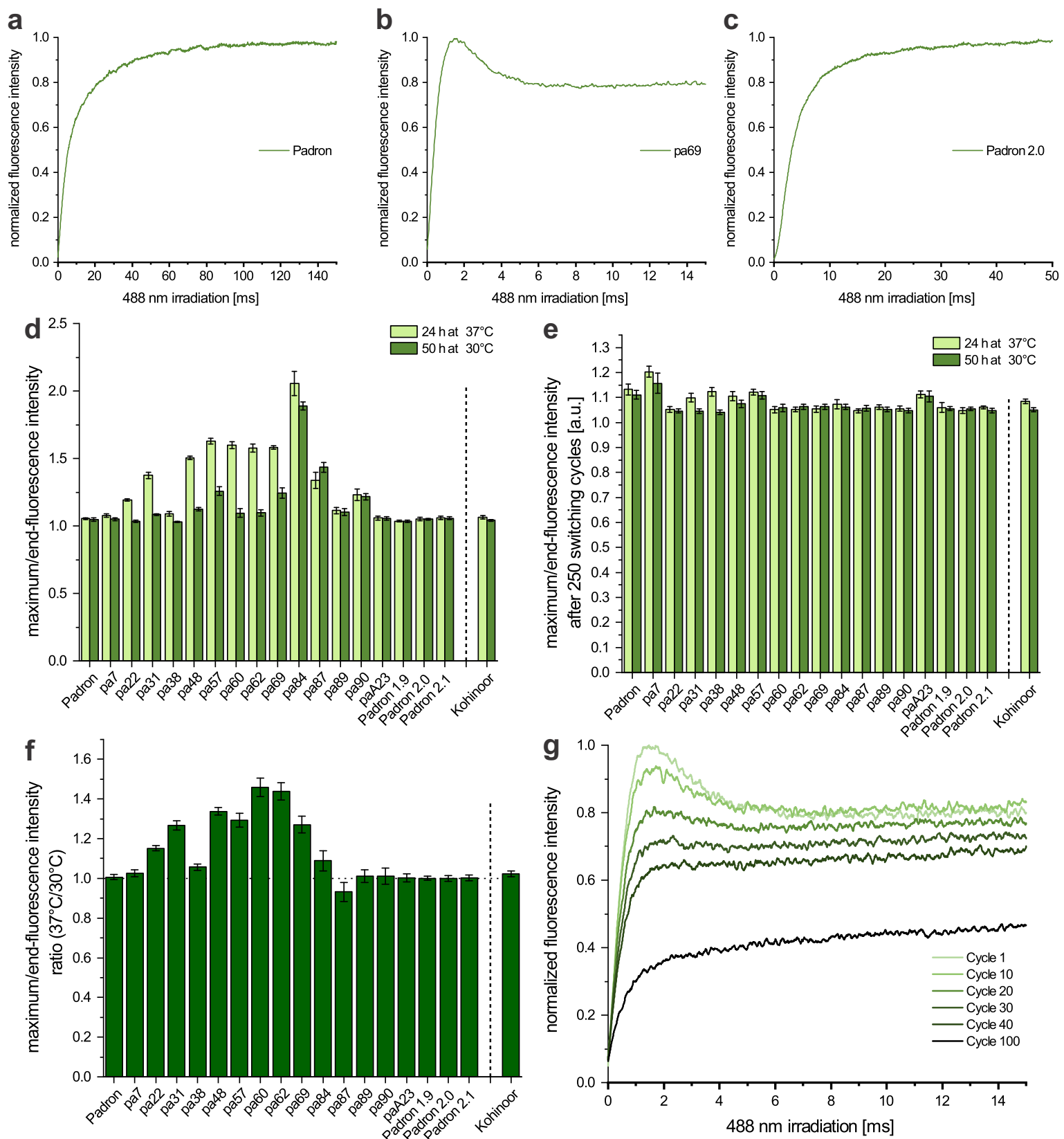

Figure 3.8: Bright intermediate state of intermediate Padron variants. Normalized representative on-switching curves of (a) Padron, (b) pa69, and (c) Padron 2.0. (d) Ratio of the maximum and end data points of all intermediate Padron variants in the second switching cycle and (e) after 250 cycles. (f) Ratios of different growth conditions of data presented in d. (g) Selected on-switching curves of pa69. E. coli colonies were measured after growth at $37^{\circ} \mathrm{C}$ for $24 \mathrm{~h}$ or at $30^{\circ} \mathrm{C}$ for $50 \mathrm{~h}$. Data represents averaged measurements of 10 colonies. Switching curves are smoothed single measurements. 


\subsection{Detailed characterization of Padron 1.9, 2.0, and 2.1}

\subsubsection{Characterization of switching characteristics}

Due to its superior performance in comparative measurements (sec. 3.3) with regards to switching fatigue (fig. 3.7), switching to the off-state (fig. 3.6) and ensemble fluorescence intensity (fig. 3.5a), Padron 2.0 was considered to be the most promising variant for live-cell nanoscopy. However, Padron 1.9 and Padron 2.1 displayed superior characteristics in single measurements, rendering them promising candidates for specialized applications: Padron 1.9 displayed higher fluorescence intensity in the on-state but had an impaired switching fatigue, suggesting its use for imaging of structures that exhibit a low fluorescence signal in fluorescence imaging. Padron 2.1 in contrast displayed lower fluorescence intensity than Padron 2.0 but efficiently switched to the off-state under simultaneous $405 \mathrm{~nm}$ and $488 \mathrm{~nm}$ irradiation. In the following section these three variants will be analyzed in detail with regards to their switching characteristics and compared to the original Padron. In addition, Kohinoor was subjected to the same detailed characterization. It was published as an improved variant of Padron during the course of this thesis, but displayed switching characteristics which were different from those of the final variants in the comparative measurements. Data were recorded at the automated screening microscope in bacterial colonies (cf. methods section 5.3.1.4).

\subsubsection{Residual off-state fluorescence intensity}

An important characteristic of RSFPs for RESOLFT nanoscopy is the residual off-state fluorescence intensity after saturated switching, which is defined as the lowest residual fluorescence that can be achieved as a result of switching in a given sample. A lower switching background results in an increased ratio of fluorescence contribution of central fluorophores to peripheral fluorophores during point-scanning RESOLFT imaging and thus improves the achievable resolution. Because all Padron variants shown did not or only barely fluoresce with $405 \mathrm{~nm}$ excitation, the off-switching could not be analyzed without interfering light of $488 \mathrm{~nm}$. Instead, an indirect approach had to be chosen to determine the saturated residual off-state fluorescence intensity. Proteins in bacterial colonies were switched to the off-state with various $405 \mathrm{~nm}$ light doses, and the residual off-state fluorescence was calculated from the consecutive on-switching curve. Data from these measurements is shown in figure 3.9, exact values of ensemble switching backgrounds are listed in table 3.1. 

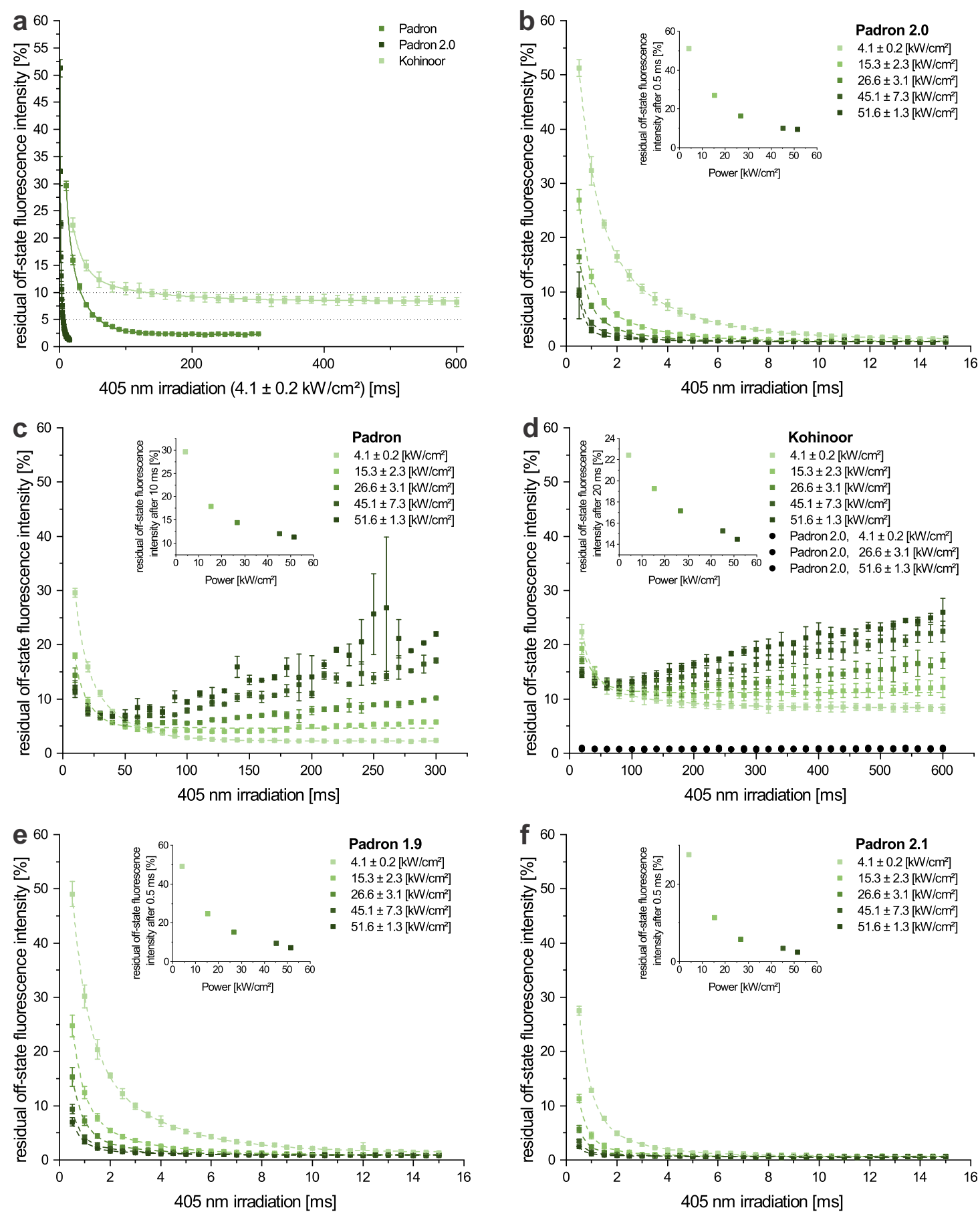

Figure 3.9: Saturated residual off-state fluorescence intensity of Padron variants. $405 \mathrm{~nm}$ light doses were modulated by means of intensity and duration, and the resulting residual off-state fluorescence intensity was calculated from the consecutive on-switching curve. (a) Overview of Padron, Padron 2.0 and Kohinoor measured at $4.1 \mathrm{~kW} / \mathrm{cm}^{2} 405 \mathrm{~nm}$ intensity. Detailed data of the modulation are shown for (b) Padron 2.0, (c) Padron, (d) Kohinoor, (e) Padron 1.9, and (f) Padron 2.1. Insets depict first data points of residual off state fluorescence intensities as a function of laser intensity. 


\section{Results}

Responsiveness to $405 \mathrm{~nm}$ irradiation varied strongly for the proteins analyzed. The new variants generated in this thesis could efficiently be switched to ensemble background values below $1 \%$ at almost all laser intensities applied (fig. 3.9a, e, f), with Padron 2.1 reaching the lowest values at all intensities with a minimum of $0.57 \pm 0.01 \%$. These values were achieved after $15 \mathrm{~ms}$ irradiation with the given intensities.

Table 3.1: Switching Background in E. coli. Irradiation times varied between samples.

\begin{tabular}{lccccc}
$\mathbf{4 0 5} \mathbf{~ n m ~}\left[\mathbf{k W} / \mathbf{c m}^{2}\right]:$ & $\mathbf{4 . 1} \pm \mathbf{0 . 2}$ & $\mathbf{1 5 . 3} \pm \mathbf{2 . 3}$ & $\mathbf{2 6 . 6} \pm \mathbf{3 . 1}$ & $\mathbf{4 5 . 1} \pm \mathbf{7 . 3}$ & $\mathbf{5 1 . 6} \pm \mathbf{1 . 3}$ \\
\hline Protein & \multicolumn{5}{c}{ Switching background $[\%]$} \\
\hline Padron & $2.27 \pm 0.02$ & - & - & - & - \\
Kohinoor & $8.42 \pm 0.63$ & - & - & - & - \\
Padron 1.9 & $1.53 \pm 0.11$ & $0.99 \pm 0.03$ & $0.92 \pm 0.03$ & $0.91 \pm 0.02$ & $0.91 \pm 0.01$ \\
Padron 2.0 & $1.41 \pm 0.09$ & $0.90 \pm 0.04$ & $0.84 \pm 0.02$ & $0.79 \pm 0.01$ & $0.84 \pm 0.08$ \\
Padron 2.1 & $0.80 \pm 0.15$ & $0.67 \pm 0.06$ & $0.62 \pm 0.03$ & $0.62 \pm 0.08$ & $0.57 \pm 0.01$ \\
\hline Padron 2.0 & $0.71 \pm 0.08$ & - & $0.76 \pm 0.05$ & - & $0.88 \pm 0.08$ \\
(Kohinoor settings) $^{*}$ & &
\end{tabular}

*values are averaged values of all data points shown in figure $3.9 \mathrm{~d}$ based on a single repetition.

Transferring these measurement conditions to Padron and Kohinoor was not possible as the irradiation duration was insufficient to reach a plateau for the switching background. Thus, irradiation was prolonged to $300 \mathrm{~ms}$ for Padron and to $600 \mathrm{~ms}$ for Kohinoor (fig. 3.9a). This revealed unfavorable $405 \mathrm{~nm}$ responses of both proteins. While the new Padron variants could be switched off more efficiently with increasing $405 \mathrm{~nm}$ duration and intensity, monotonous background decrease was displayed by Padron and Kohinoor only at the lowest laser intensity applied, reaching switching backgrounds of $2.27 \pm 0.02$ and $8.42 \pm 0.63 \%$, respectively (tab. 3.1). Higher intensities resulted in an initial decrease of residual off-state fluorescence which was reversed to higher values with prolonged irradiation, suggesting detrimental effects of high $405 \mathrm{~nm}$ light doses (fig. 3.9c, d). Because the irradiation doses varied so strongly, Padron 2.0 was measured under the same conditions as Kohinoor to allow for a direct comparison (fig. 3.9d). The resulting data suggested no detrimental effect of prolonged $405 \mathrm{~nm}$ irradiation on the switching background of Padron 2.0 (tab. 3.1).

\subsubsection{On-Switching kinetics}

After the switching background, switching times for the activation of Padron variants from the off-state were analyzed. Fast switching RSFPs allow for shorter pixel dwell times during RESOLFT imaging. This is a crucial parameter because the dwell times of RESOLFT nanoscopy with RSFPs are comparatively long compared to other super-resolution methods like STED [124, 170]. Slow RSFPs can require pixel dwell times of up to 100 milliseconds [144], which makes RESOLFT imaging of fast dynamics in living cells impossible.

To address kinetics of switching from the off- to the on-state, Padron variants were activated with different $488 \mathrm{~nm}$ laser intensities. For this purpose, proteins were switched to $5 \%$ residual fluorescence intensity, with the exception of Kohinoor, which was switched to $10 \%$. 

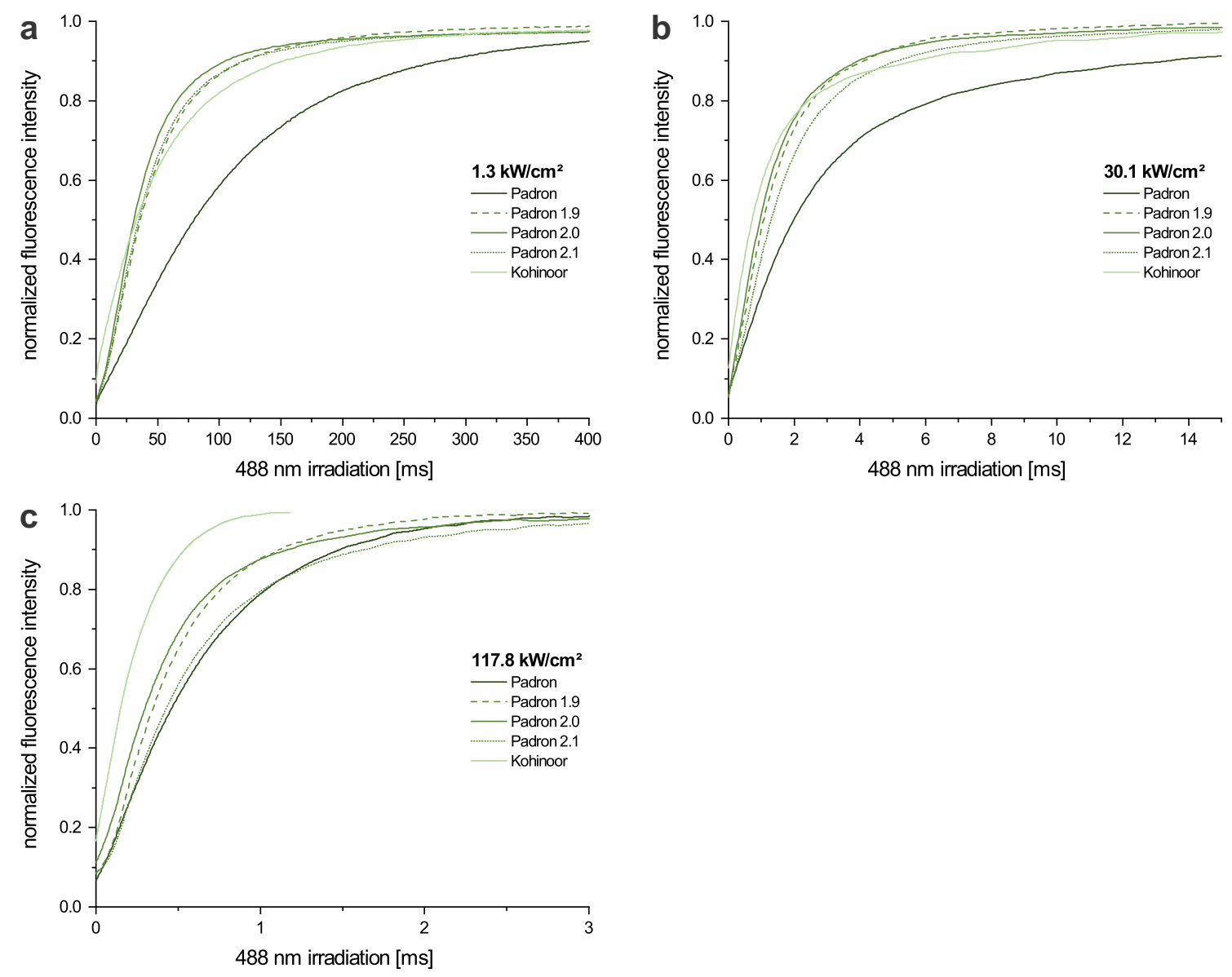

Figure 3.10: On-Switching kinetics of Padron variants. Proteins were switched to $5 \%$ (Kohinoor: 10\%) residual fluorescence and switched back to the on-state with 8 different $488 \mathrm{~nm}$ laser intensities. Three representative laser intensities are shown. (a) Low $\left(1.3 \mathrm{~kW} / \mathrm{cm}^{2}\right)$, (b) medium $\left(30.1 \mathrm{~kW} / \mathrm{cm}^{2}\right)$, and (c) high $\left(117.8 \mathrm{~kW} / \mathrm{cm}^{2}\right)$ intensity.
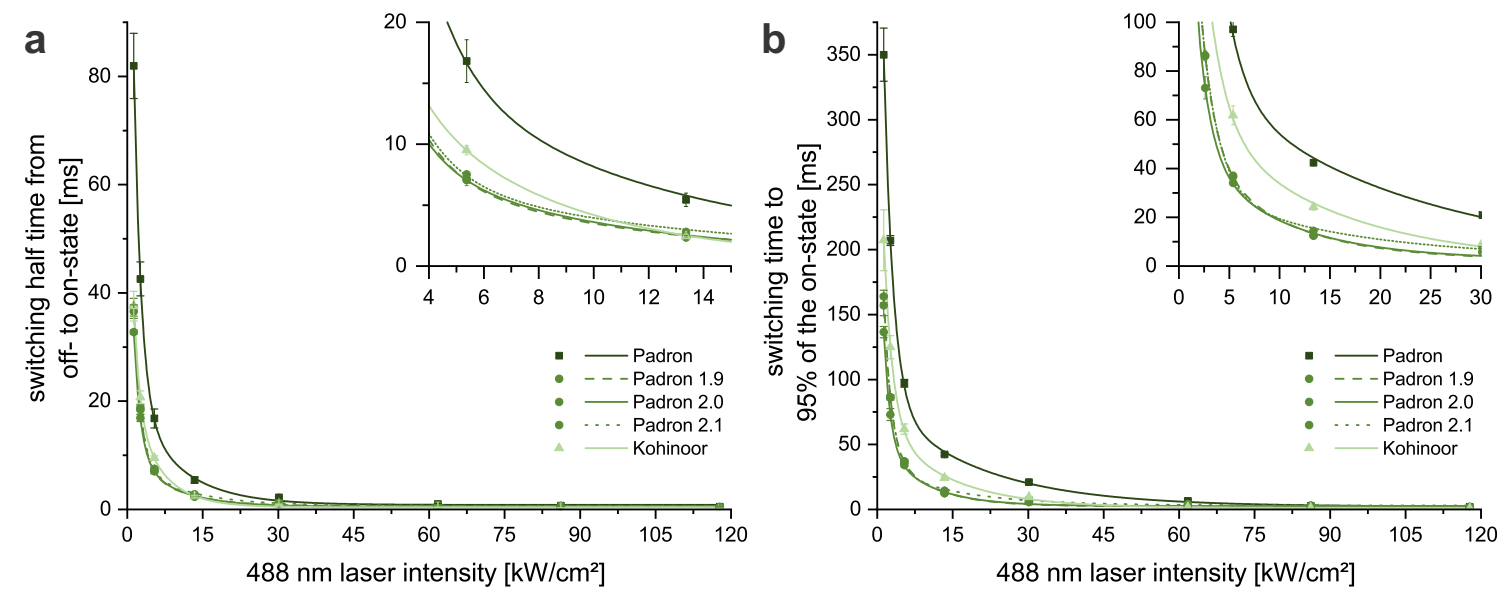

Figure 3.11: On-Switching kinetics in bacterial colonies as a function of laser intensity. (a) Switching half-time from off- to on-state. (b) Switching time to $95 \%$ of the on-state fluorescence intensity, Padron 1.9 and 2.1 are overlapping. 


\section{Results}

This was due to the fact that Kohinoor could not reach residual fluorescence intensities below $8 \%$ in bacterial colonies (sec. 3.4.1.1). Proteins were then switched back to the on-state at 8 different $488 \mathrm{~nm}$ laser intensities; the detailed curves for all proteins are depicted in figure 7.1 in the appendix.

Figure 3.10 compares on-switching curves of all variants for representative laser intensities, in the following referred to as low $\left(1.3 \mathrm{kw} / \mathrm{cm}^{2}\right)$, medium $\left(30.1 \mathrm{kw} / \mathrm{cm}^{2}\right)$, and high $\left(117.8 \mathrm{kw} / \mathrm{cm}^{2}\right)$. The original Padron displayed the slowest kinetics for low and medium intensity (fig. 3.10a, b), whereas the new Padron variants had a very similar kinetic response to $488 \mathrm{~nm}$ irradiation. Kohinoor is only marginally slower at these intensities. However, at high intensities this order was abrogated (fig. 3.10c). Padron and Kohinoor kinetics scaled faster with increased laser intensities rendering Kohinoor the fastest protein and leveling Padron 2.1 with the original Padron.

Table 3.2: Switching half-times $[\mu s]$ from off- to on-state.

\begin{tabular}{lccccc} 
kw $/ \mathbf{c m}^{2}$ & Padron & Padron 1.9 & Padron 2.0 & Padron 2.1 & Kohinoor \\
\hline 1.3 & 81936 & 37353 & 32770 & 36556 & 37560 \\
2.6 & 42593 & 18430 & 16900 & 18776 & 20720 \\
5.4 & 16816 & 7066 & 7090 & 7516 & 9503 \\
13.4 & 5440 & 2340 & 2413 & 2806 & 2433 \\
30.1 & 2223 & 1156 & 1040 & 1403 & 916 \\
61.7 & 993 & 686 & 550 & 846 & 406 \\
86.2 & 690 & 506 & 463 & 646 & 276 \\
117.8 & 493 & 390 & 346 & 476 & 200 \\
\hline
\end{tabular}

Table 3.3: Switching times $[\mu \mathrm{s}]$ to $95 \%$ of the full on-state. kw $/ \mathrm{cm}^{2}$ Padron Padron 1.9 Padron 2.0 Padron 2.1 Kohinoor

\begin{tabular}{lccccc}
\hline 1.3 & 350036 & 163880 & 136523 & 157060 & 207170 \\
2.6 & 206973 & 86190 & 73056 & 86430 & 124870 \\
5.4 & 97130 & 37020 & 34096 & 36696 & 61826 \\
13.4 & 42366 & 12453 & 12603 & 14420 & 24166 \\
30.1 & 20933 & 5710 & 5850 & 7736 & 9496 \\
61.7 & 6620 & 2856 & 2890 & 4203 & 1826 \\
86.2 & 2736 & 2000 & 2283 & 3076 & 1066 \\
117.8 & 1926 & 1460 & 1583 & 2133 & 733 \\
\hline
\end{tabular}

Color gradients are applied row-wise. Largest/slowest values are colored in light green, smallest/fastest values are colored in dark green. Standard deviations are listed in tables 7.1 and 7.2 in section 7.2 .

These relations became clearer when switching half-times and switching time to $95 \%$ of the saturated on-state were considered. Figure 3.11 gives a good estimate of absolute differences of these values, whereas tables 3.2 and 3.3 list the respective values. Rows in these tables are gradually color coded in a way that the lowest value at each intensity is shaded with a light green while the highest and thus fastest value is shaded with a dark green. This clearly visualizes how kinetic relations changed with respect to laser intensities.

While activation half-times of the new Padron variants and Kohinoor were rather similar, half- 
times of the original Padron were approximately twice as long at low to medium intensities. At higher intensities, this difference was in part attenuated (tab. 3.2). Interestingly, Kohinoor had slightly slower half-times than the new Padron variants at low laser intensities but in turn switched faster at intensities above $13.4 \mathrm{~kW} / \mathrm{cm}^{2}$ (inset of fig. 3.11a). The same statement applies to switching to $95 \%$ of the full on-state (inset of fig. 3.11b), although only at intensities above $61.7 \mathrm{~kW} / \mathrm{cm}^{2}$ (tab. 3.3). Differences between new Padron variants and Kohinoor at low intensities were larger here, but the original Padron was still the protein with regards to the activation half-time, while Padron 2.1 was the only variant that needed more time to switch to $95 \%$ of the on-state at high intensities in comparison to Padron.

\subsubsection{Switching fatigue}

After characterization of ensemble switching background and kinetics, switching fatigue performances of the new Padron variants were addressed. Switching fatigue is a measure for the integrated loss of fluorescence during repeated switching as a result of photobleaching and other processes. The ability to sustain fluorescence emission and switching capabilities while performing a plethora of switching cycles is a key characteristic for the applicability of an RSFP in RESOLFT microscopy. Switchable fluorescent proteins that endure a large number of cycles allow for smaller pixel sizes, which are necessary to resolve nanoscale structures, or enable imaging of multiple frames in RESOLFT mode, which is a requirement for the visualization of dynamic processes in living cells.

To characterize this property, proteins were switched in bacterial colonies for 1000 cycles. Light doses applied were different for every protein to factor in differing kinetic responses to 405 and $488 \mathrm{~nm}$ irradiation. To better represent the switching fatigue in response to state saturation during RESOLFT microscopy, Padron variants were switched to $5 \%$ residual fluorescence (Kohinoor: $10 \%$ ) with $405 \mathrm{~nm}$ at $3.6 \mathrm{~kW} / \mathrm{cm}^{2}$ and subsequently back to $95 \%$ of the on-state fluorescence intensity; detailed irradiation times are given in table 5.8 in the methods section 5.3.1.4.

Results of switching fatigue measurements are depicted in figures 3.12a and 3.12b. The new Padron variants outperformed the original Padron and Kohinoor in every aspect. While Padron 1.9 was the least performing of the new variants presented here (section 3.3.1), it still was able to complete significantly more switching cycles than Padron and Kohinoor. The latter two reached $50 \%$ on-state fluorescence intensity after only 19 and 37 cycles at low and 13 and 28 cycles at high $488 \mathrm{~nm}$ intensity, respectively, whereas Padron 1.9 was bleached to $50 \%$ after 433 and 300 cycles (tab. 3.4). Padron 2.0 reached this fluorescence intensity after 990 and 847 cycles, outperforming Kohinoor with an approximately 25- and 30-fold higher number of cycles at the given conditions. This performance was based in part on the distinct behavior of Padron 2.0 with regards to switching fatigue: During cycling, this variant displayed an increase of fluorescence intensity at the start of the measurement (fig. 3.12a, b). This effect was also visible for Padron 2.1 at low $488 \mathrm{~nm}$ intensity, allowing for more than 1000 cycles to be completed before reaching $50 \%$ fluorescence intensity, but was absent at 

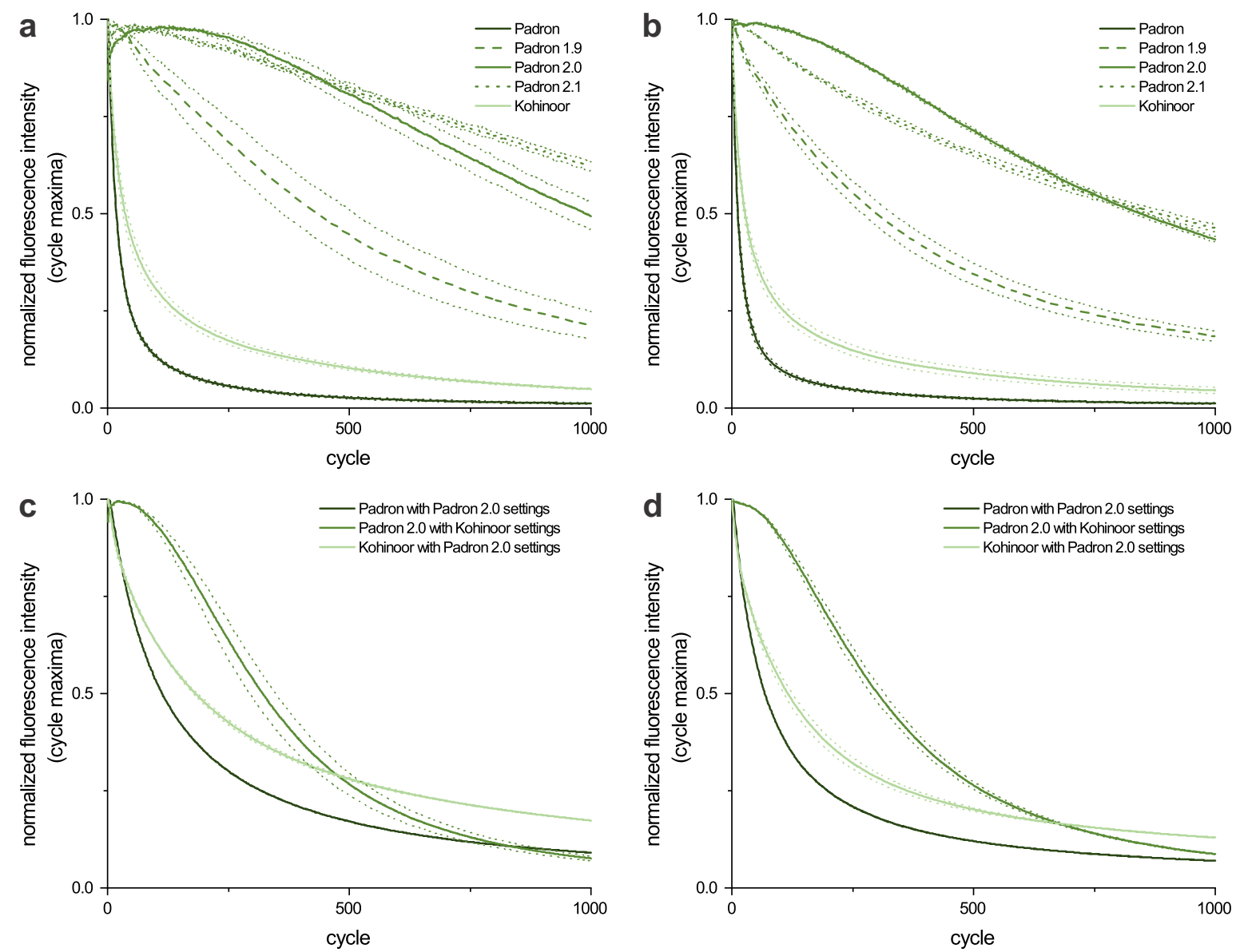

Figure 3.12: Switching fatigue in bacterial colonies. Padron variants were switched to $5 \%$ residual off-state fluorescence intensity (Kohinoor: 10\%) and back to $95 \%$ of the fluorescent on-state 1000 times at two different $488 \mathrm{~nm}$ intensities. (a, b) On-state fluorescence intensity after switching with (a) low $488 \mathrm{~nm}$ intensity $\left(2.6 \mathrm{~kW} / \mathrm{cm}^{2}\right)$ and (b) with high $488 \mathrm{~nm}$ intensity $\left(30.1 \mathrm{~kW} / \mathrm{cm}^{2}\right)$. (c, d) Comparative measurements with (c) low and (d) high $488 \mathrm{~nm}$ intensity.

high intensity where $50 \%$ intensity was reached after 847 cycles. Interestingly, this activation effect compensated switching fatigue of Padron 2.0 compared to Padron 2.1 at low cycle numbers. However, this relation was dissipated after more cycles due to the slower fluorescence decay of Padron 2.1 (fig. 3.12a, b).

Although the method used here adequately simulated repeated cycling during RESOLFT microscopy, one has to consider the different light doses applied as Kohinoor and Padron needed significantly more time to switch to the on- or off-state. To estimate this influence, Padron and Kohinoor were measured with the settings used for Padron 2.0, which in turn was measured with Kohinoor irradiation doses (fig. 3.12c, d). This approach had a pronounced effect on the outcome: Padron 2.0 could undergo approximately $65 \%$ fewer cycles before reaching $50 \%$ fluorescence intensity at low and high $488 \mathrm{~nm}$ intensity. Conversely, the number of cycles was increased 4-6-fold for Padron and Kohinoor (tab. 3.4). Nevertheless, Padron 2.0 still outperformed both proteins. In addition, switching of Padron and Kohinoor was far from 
being complete with these truncated irradiation times: Both variants only switched to approximately 30-40\% residual fluorescence intensity, a value that is insufficient for RESOLFT imaging because fluorescence contribution of peripheral, incompletely switched fluorophores would be too high to allow for the distinguishment of saturated states that confines fluorescence contribution to a volume smaller than the diffraction limit. Figure 7.2 in the appendix depicts the switching fatigue data for each protein individually and also shows the residual fluorescence of each cycle.

Table 3.4: Switching fatigue and photobleaching performance of Padron variants.

\section{Protein}

488 nm intensity:
Number of Cycles at $50 \%$ maximal intensity*

Padron

Kohinoor

Padron 1.9

Padron 2.0

Padron 2.1 $2.6\left[\mathrm{~kW} / \mathrm{cm}^{2}\right]$

$30.1\left[\mathrm{~kW} / \mathrm{cm}^{2}\right]$

$19\left(113^{a}\right)$

$37\left(182^{a}\right)$

433

$990\left(321^{b}\right)$

$>1000$

Time to bleach to $50 \%$ of maximal intensity ${ }^{+}[\mathrm{s}]$ $2.4 \pm 0.3\left[\mathrm{~kW} / \mathrm{cm}^{2}\right] \quad 30.8 \pm 1.5\left[\mathrm{~kW} / \mathrm{cm}^{2}\right]$

$\begin{array}{cc}11.4 & 1.3 \\ 21.5 & 1.6 \\ 29.0 & 1.1 \\ 67.4 & 3.7 \\ 105.8 & 5.3\end{array}$

*first cycle below $50 \%$ after normalization ${ }^{a}$ measured with Padron 2.0 settings ${ }^{b}$ measured with Kohinoor settings ${ }^{+}$first data point below $50 \%$ of maximal intensity (sampling rates: $0.63 \mathrm{~s}$ for lower, $0.18 \mathrm{~s}$ for higher power bleaching measurements)

\subsubsection{Photobleaching}

Aside from switching fatigue, photobleaching, which is the bleaching in response to fluorescence excitation, is a key characteristic of RSFPs determining their applicability for microscopy. For Padron as a positively switching fluorescence protein this parameter is of special interest. While negatively switching fluorescence proteins necessarily cycle between on- and off-state even in diffraction-limited applications, positively switching FPs can be used like non-switchable ones, rendering any additional activation step obsolete.

To address photobleaching of Padron variants, fluorescence decay in response to $488 \mathrm{~nm}$ irradiation was measured in bacterial colonies at low $\left(2.3 \pm 0.3 \mathrm{~kW} / \mathrm{cm}^{2}\right)$ and high $(30.8 \pm$ $1.5 \mathrm{~kW} / \mathrm{cm}^{2}$ ) laser intensity (fig. 3.13). At both conditions, Padron was most sensitive to photobleaching and was bleached to $50 \%$ of the maximal intensity after $11.4 \mathrm{~s}$ at low and after $1.3 \mathrm{~s}$ at high intensity (tab. 3.4). At low intensity, this value was increasingly higher from Kohinoor over Padron 1.9 and Padron 2.0 to Padron 2.1, with the latter one fluorescing nearly 10 times longer before it was bleached to $50 \%$ fluorescence intensity. This order was similar at high intensities, with the exception of Padron 1.9, which performed slightly worse than the original Padron (1.1 s versus 1.3 s). In addition, Padron 2.1 sustained $488 \mathrm{~nm}$ excitation only approximately 4 times longer than Padron. Padron 2.0 could be excited approximately 6 and 3 times longer than Padron before it was bleached to $50 \%$ of its initial fluorescence intensity. 

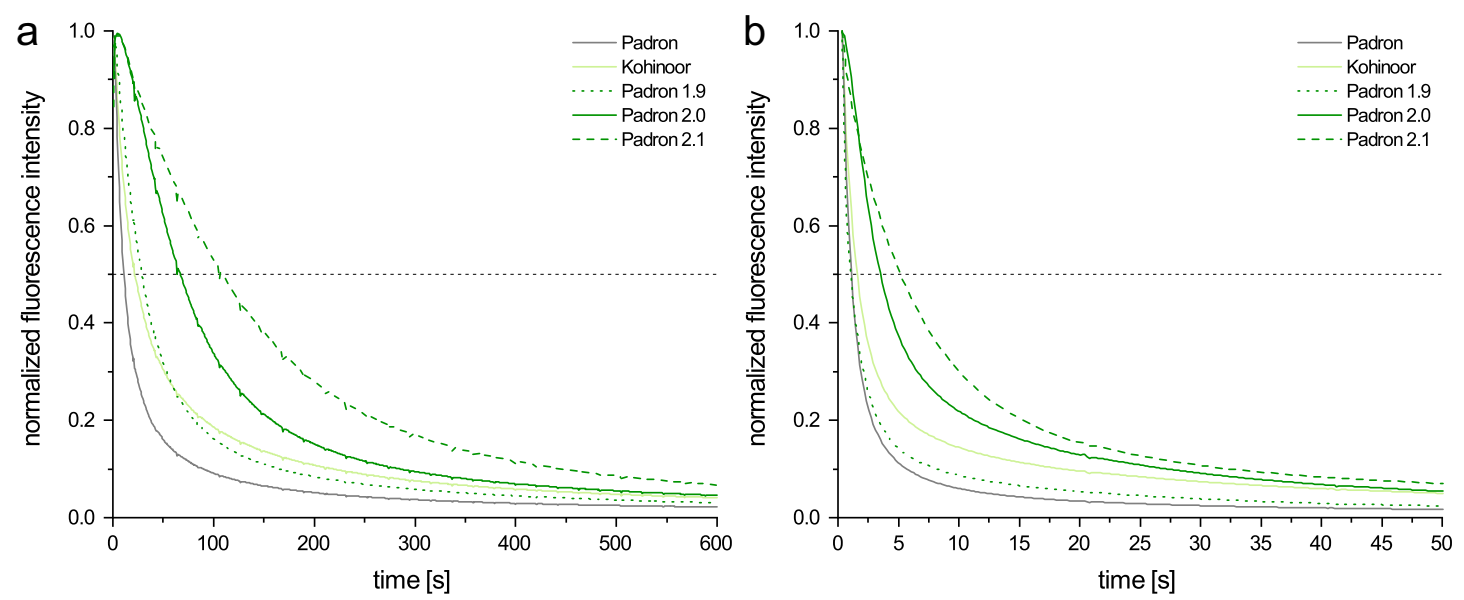

Figure 3.13: Photobleaching in bacterial colonies. Fluorescence decay due to photobleaching with $488 \mathrm{~nm}$ light was measured in bacterial colonies. (a) $10 \mathrm{~min}$ at $2.3 \pm$ $0.3 \mathrm{~kW} / \mathrm{cm}^{2}$, (b) $50 \mathrm{~s}$ at $30.8 \pm 1.5 \mathrm{~kW} / \mathrm{cm}^{2}$.

\subsubsection{Switching background with simultaneous illumination}

To fully employ the proposed advantages of positively switching fluorescent proteins in RESOLFT microscopy, the ability to suppress $488 \mathrm{~nm}$ induced return to the on-state with $405 \mathrm{~nm}$ light is essential. Suppression in this case refers to the apparent ensemble switching state of fluorophores: When both wavelength are applied, single molecules are expected to cycle between the on- and off-state. If the rate for $405 \mathrm{~nm}$ induced off-switching is much higher than the rate for $488 \mathrm{~nm}$ dependent activation, the ensemble of RSFPs will adapt an equilibrium close to the off-state. Proteins with such a ratio of on- and off-switching kinetics allow for peripheral suppression of fluorophores with a doughnut-shaped beam during readout, resulting in prolonged fluorescence probing of a volume smaller than the diffraction limit. To address this feature for new Padron variants, proteins were switched in bacterial colonies with simultaneous irradiation. $488 \mathrm{~nm}$ induced activation of proteins was suppressed at two different intensities with increasing $405 \mathrm{~nm}$ laser intensities. As it was shown in section 3.4.1.1, the $405 \mathrm{~nm}$ response was different for Padron, Kohinoor, and the new variants. Irradiation duration was thus varied for these measurements as well, detailed parameters are listed in table 5.9 in section 5.3.1.4.

Figure 3.14 shows the results of these measurements. While the new Padron variants could be switched to residual off-state fluorescence intensities below $5 \%$ with all $405 \mathrm{~nm}$ intensities employed at low simultaneous $488 \mathrm{~nm}$ irradiation of $1.1 \mathrm{~kW} / \mathrm{cm}^{2}$ (fig. $3.14 \mathrm{a}$ ), high $488 \mathrm{~nm}$ intensity of $15.0 \mathrm{~kW} / \mathrm{cm}^{2}$ could only be compensated to similar residual off-state fluorescence intensities with $405 \mathrm{~nm}$ irradiation above $25 \mathrm{~kW} / \mathrm{cm}^{2}$ for Padron 1.9 and 2.0 (fig. $3.14 \mathrm{~b}$ ). Padron 2.1 performed exceptionally well even at lower $405 \mathrm{~nm}$ intensities reaching residual fluorescence below $5 \%$ with approximately $10 \mathrm{~kW} / \mathrm{cm}^{2}$. This was in accordance with the comparative measurements shown in figure 3.6b.

Padron and Kohinoor on the other hand could not be reliably switched off at any of the intensity combinations employed. On the contrary, residual fluorescence appeared to increase with 

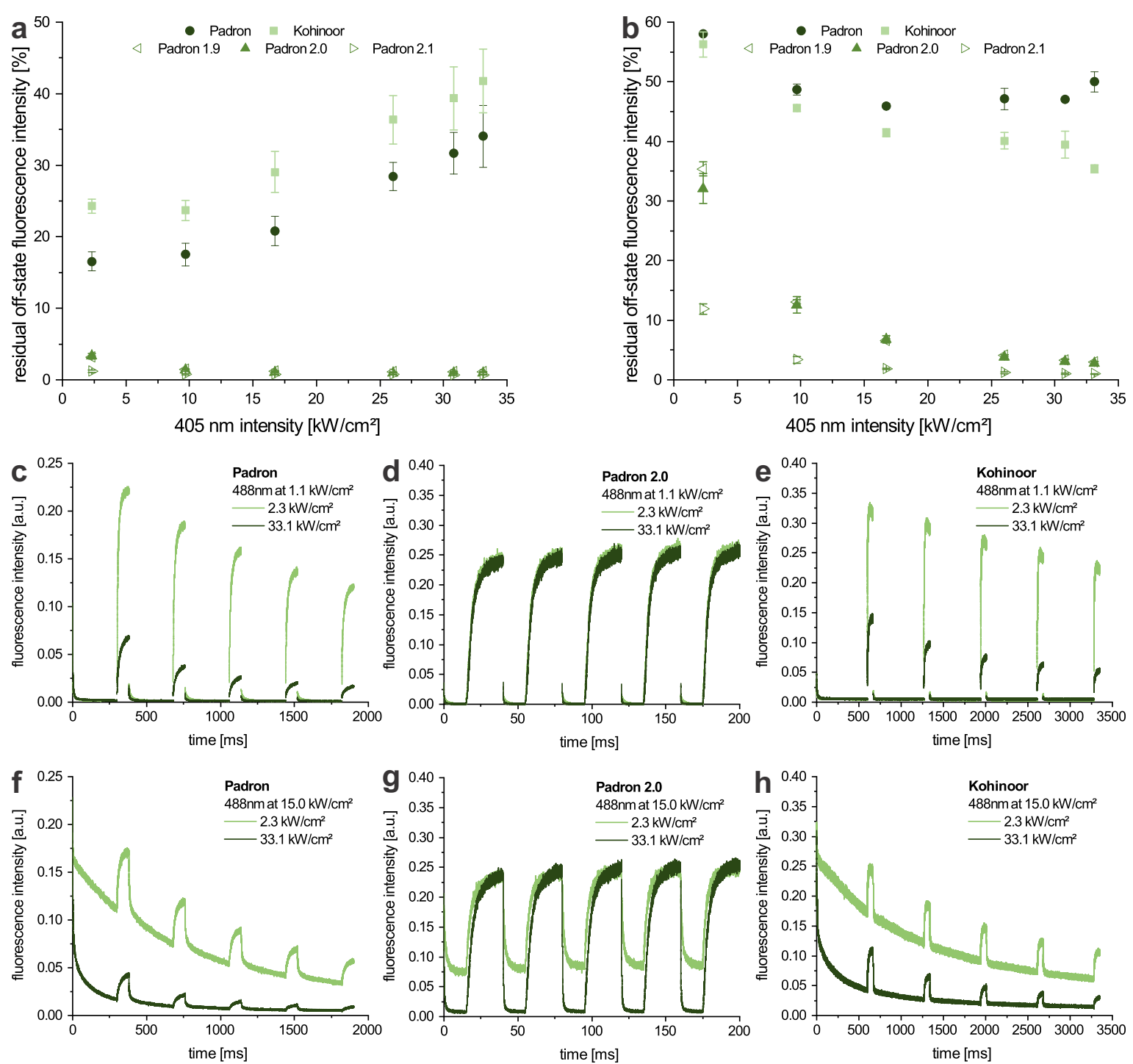

Figure 3.14: Residual off-state fluorescence intensity with simultaneous 405 and $488 \mathrm{~nm}$ irradiation. (a) Residual off-state fluorescence intensity as a function of $405 \mathrm{~nm}$ laser intensity with simultaneous $488 \mathrm{~nm}$ irradiation at $1.1 \mathrm{~kW} / \mathrm{cm}^{2}$ and (b) at $15.0 \mathrm{~kW} / \mathrm{cm}^{2}$. Associated switching curves for different $405 \mathrm{~nm}$ intensity combinations are shown for (c) Padron, (d) Padron 2.0, and (e) Kohinoor with $488 \mathrm{~nm}$ at $1.1 \mathrm{~kW} / \mathrm{cm}^{2}$ or (f) Padron, (g) Padron 2.0, and (h) Kohinoor with $488 \mathrm{~nm}$ at $15.0 \mathrm{~kW} / \mathrm{cm}^{2}$.

higher $405 \mathrm{~nm}$ intensities (fig. 3.14a, b). This was most likely due to long irradiation times applied: With simultaneous 405 and $488 \mathrm{~nm}$ irradiation, RSFPs were subjected to constant cycling between on- and off-state, evoking switching fatigue and photobleaching as a consequence of constant excitation. While the new Padron variants apparently were not affected under the given conditions (fig. $3.14 \mathrm{~d}, \mathrm{~g}$ ), these parameters were detrimental to Padron and Kohinoor. At low $488 \mathrm{~nm}$ intensity, $405 \mathrm{~nm}$ irradiation apparently was sufficient to reach a steady-state but already induced bleaching as a result of switching fatigue after only 5 cycles (fig. 3.14c, e). At high $488 \mathrm{~nm}$ intensity, no steady-state formation was visible. Instead, the decay during simultaneous irradiation resembled the bleaching that was observable in the 


\section{Results}

activated state (fig. 3.14f, h). This data suggested that the on-state transition of Padron and Kohinoor cannot be suppressed by simultaneous irradiation with $405 \mathrm{~nm}$ without severe photodamage.

After all, it could be shown that Padron 2.0 was a significant improvement over Padron and Kohinoor with regards to the switching performance in E. coli. Switching to the off-state and switching fatigue, two essential parameters for the application of RSFPs in RESOLFT microscopy, were significantly improved. Padron 1.9 was brighter than Padron 2.0 but more prone to bleach during repeated switching, whereas Padron 2.1 was displayed lower fluorescence intensity but performed better with regards to switching to the off-state. Nevertheless, Padron 1.9 and 2.1 might be useful for niche applications where their features are of special interest.

\subsubsection{In vitro switching and metastability}

Because the new Padron variants displayed greatly improved switching characteristics in bacterial colonies, all 5 proteins analyzed in the previous section were purified from bacterial cultures. Isolated proteins samples showed good purity (fig. 7.3 in the appendix) and were characterized in vitro (cf. methods sec. 5.5.1) in order to gain insight into protein characteristics independent from expression and maturation.

For this purpose, proteins were switched in standard protein buffer at $\mathrm{pH} 7.5$, and fluorescence was observed over time. By initially switching proteins to the off-state prior to recording the on-state and vice versa, fluorescence intensities of all states allowed for calculation of the ensemble switching background and the equilibrium ground-state fluorescence relative to the on-state. The exact values obtained from these measurements are listed in table 3.5. These results illustrate that the relative ground-state fluorescence intensity was changed during mutagenesis. While only approximately $10 \%$ of Padron fluorophores were fluorescent in the equilibrium, $50-70 \%$ of the fluorophores of the new Padron variants resided in the on-state. Kohinoor was found to reside nearly entirely in the on-state in its equilibrium. Interestingly, Padron 2.1 had different equilibrium state fluorescence intensities depending on the sequence in which states were recorded, which had no influence on the other variants. This resulted from the fact that absolute on-state fluorescence intensity of Padron 2.1 was higher if samples were switched to the on-state from the off-state instead of the equilibrium ground-state.

Ensemble switching background for Padron and the new variants was higher than measured in E. coli. In contrast, Kohinoor showed improved switching background compared to previous measurements. One possible reason for this was presumably the light intensity used for switching. While switching at the automated screening microscope was facilitated with light in the $\mathrm{kW} / \mathrm{cm}^{2}$ range, the intensities for in vitro switching were in the $\mathrm{mW} / \mathrm{cm}^{2}$ range, suggesting that Kohinoor could be switched off more efficiently at low intensities. Such a behavior would have implications for RESOLFT microscopy, as low intensities in the $\mathrm{mW} / \mathrm{cm}^{2}$ would result in longer pixel dwell times. An impaired performance at higher intensities would limit the acceleration of imaging dwell times. 
Table 3.5: Relative in vitro ground-state fluorescence intensity and ensemble switching background.

\begin{tabular}{lccccc} 
Protein & \multicolumn{2}{c}{ ground-state* [\%] } & \multicolumn{2}{c}{ off-state [\%] } & on-state [\%] \\
\hline & On $\rightarrow$ Off & Off $\rightarrow$ On & On $\rightarrow$ Off & Off $\rightarrow$ On & \\
\hline Padron & $9.6 \pm 0.1$ & $8.1 \pm 1.4$ & $4.1 \pm 0.3$ & $3.9 \pm 0.3$ & 100 \\
Padron 1.9 & $52.6 \pm 0.5$ & $52.5 \pm 0.8$ & $2.2 \pm 0.0$ & $2.0 \pm 0.0$ & 100 \\
Padron 2.0 & $65.5 \pm 2.0$ & $67.9 \pm 2.2$ & $1.9 \pm 0.1$ & $1.8 \pm 0.0$ & 100 \\
Padron 2.1 & $82.3 \pm 10.3$ & $56.3 \pm 4.9$ & $2.1 \pm 0.3$ & $1.5 \pm 0.1$ & 100 \\
Kohinoor & $96.6 \pm 1.2$ & $96.8 \pm 2.2$ & $2.1 \pm 0.1$ & $1.9 \pm 0.1$ & 100 \\
\hline
\end{tabular}

*equilibrium state of an approx. $20 \mu \mathrm{M}$ solution at room temperature and $\mathrm{pH} 7.5$

With regards to metastability, that is, the ability to maintain the on- or off-state after switching for a prolonged time, all proteins performed well. Although Padron fluorescence intensity declined over the course of $5 \mathrm{~h}$ of the measurement to almost $50 \%$ of the on-state, all other variants resided in the on-state for the time observed. Nevertheless, the new variants and Kohinoor displayed a small initial decrease to approximately $95 \%$ (fig. 3.15a). The same phenomenon was observable for the on-state. Over the full course of the measurement however, Padron, Padron 1.9, and Padron 2.0 resided in the off-state, which was close to its equilibrium for Padron. Kohinoor and Padron 2.1 on the other hand displayed a slow return to the on-state.
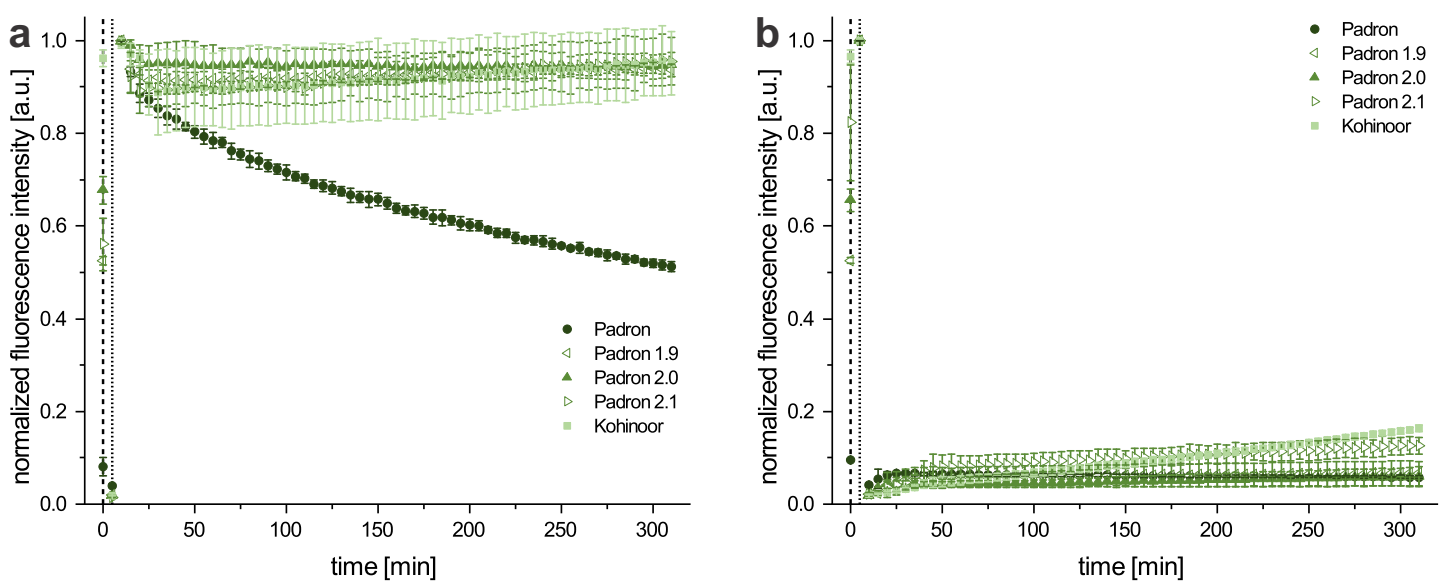

Figure 3.15: In vitro metastability of switching states. Purified Padron variants were switched and fluorescence intensity was repeatedly probed for $6 \mathrm{~h}$. (a) On-state metastability after switching proteins off- and on. (b) Off-state metastability after switching the protein on- and off. Initial data points are ground-state fluorescence intensities after equilibration to room temperature, intensities were normalized to the first on-state value.

While the changes of fluorescent states over time, where they were present, might have an influence on long-term applications, they are negligible for application of proteins for superresolution microscopy. This is especially true when one considers the time-scale at which the relaxation occurs. 


\subsubsection{Spectral properties}

\subsubsection{Absorption and emission spectra of switching states}

After switching of the new Padron variants had been established in vitro, a detailed analysis of spectral properties was conducted (cf. methods sec. 5.5.3). Similar to the experimental procedure for the characterization of metastability, absorption spectra of the equilibrium ground-state as well as on- and off-states were recorded. Altered spectral ranges can be a sign of changed dynamics in the chromophore pocket [99]. For this purpose, proteins were switched with the same intensities applied before in standard protein buffer $\mathrm{pH} 7.5$.

Table 3.6: Spectral properties of Padron variants at $\mathrm{pH} 7.5$.

\begin{tabular}{lccccc} 
Protein & \multicolumn{2}{c}{$\begin{array}{c}\text { Abs. Max } \\
{[\mathbf{n m}]}\end{array}$} & $\begin{array}{c}\text { Exc. max } \\
{[\mathbf{n m}]}\end{array}$ & $\begin{array}{c}\text { Em. max } \\
{[\mathbf{n m}]}\end{array}$ & $\begin{array}{c}\text { Em. max } \\
{[\mathbf{n m}]}\end{array}$ \\
\hline & on-state & off-state & \multicolumn{2}{c}{ equilibrium } & on-state \\
\hline Padron & 398,504 & 505 & 502 & 522 & 519 \\
Padron 1.9 & 386,496 & 504 & 493 & 516 & 514 \\
Padron 2.0 & 384,495 & 498 & 492 & 516 & 513 \\
Padron 2.1 & 387,489 & 496 & 484 & 513 & 511 \\
Kohinoor & 388,496 & 496 & 496 & 518 & 514 \\
\hline \multicolumn{5}{c}{ abs., absorption; exc., excitation; em., emission }
\end{tabular}

Figure 3.16 depicts absorption spectra of all 3 states. All Padron variants measured displayed a single absorption peak in the off-state, which is attributed to the deprotonated transchromophore [61, 140]. While this peak had its maximum at 505 and $504 \mathrm{~nm}$ for Padron and Padron 1.9, respectively, it was blue-shifted for Padron 2.0, Padron 2.1 and Kohinoor to 496-498 nm (tab. 3.6). Exciting Padron variants in this state with $488 \mathrm{~nm}$ light initiated a decrease of the deprotonated peak and gave rise to a second peak in the lower VIS range. The latter peak is attributed to the protonated form of the chromophore suggesting that both states of the chromophore are present in the on-state, where the chromophore adopts a cis-conformation $[61,140]$. As a result of the mutagenesis, the ratio of both peaks and thus the on-state equilibrium of protonated and deprotonated chromophores changed. While the deprotonated peak had an approximately twofold maximum peak absorption compared to the protonated peak for Padron (fig. 3.16a), this ratio was inverted in Kohinoor (fig. 3.16b) and, to lesser extent, in Padron 2.0 (fig. 3.16d). For Padron 1.9 and Padron 2.1, both peaks had a similar maximal peak absorption (fig. 3.16c, e).

Peak positions in the on-state were shifted compared to Padron as well. With the latter showing maximal absorption of the protonated peak at $398 \mathrm{~nm}$, this value was blue-shifted for all other proteins by $10-14 \mathrm{~nm}$. Interestingly, the peak position of the deprotonated peak in the on-state was similar to its position in the off-state for Padron, Padron 2.0, and Kohinoor but blue-shifted by 7 and $6 \mathrm{~nm}$ for Padron 1.9 and 2.1, respectively (tab. 3.6).

The peak ratios of the ground-state were situated between on- and off-state and tendentiously in accordance with the equilibrium state shown in table 3.5. However, Padron 2.1 was 

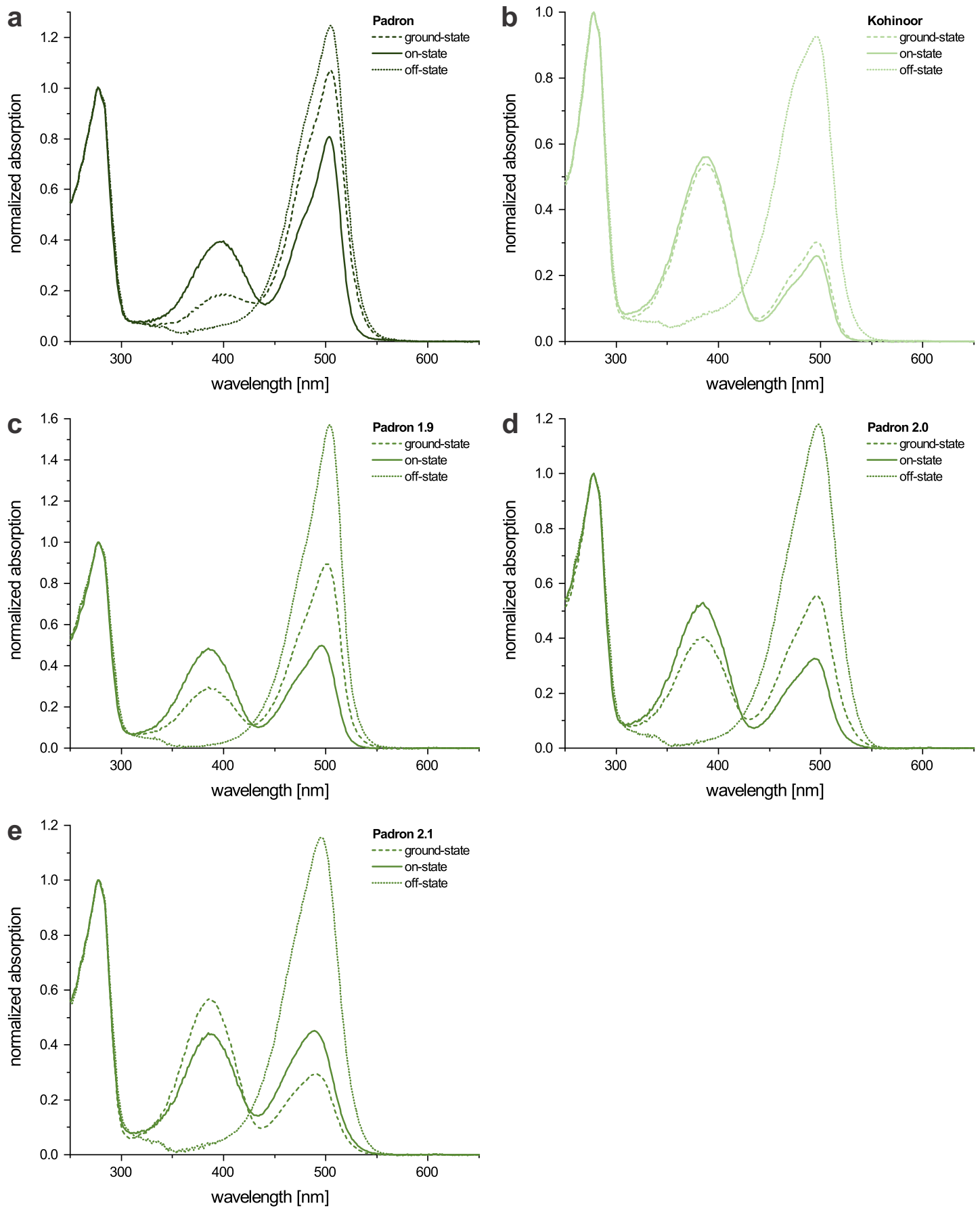

Figure 3.16: Absorption spectra of switching states. Proteins were switched in a cuvette and absorption spectra were recorded for (a) Padron, (b) Kohinoor, (c) Padron 1.9, (d) Padron 2.0, and (e) Padron 2.1. Padron 2.1 reproducibility was problematic, therefore selected spectra are shown for this variant. Spectra were normalized to the absorption of aromatic amino acids at $280 \mathrm{~nm}$. 


\section{Results}

an exception from this relation (fig. 3.16e). This was unexpected as the lowest absorption by the deprotonated peak was expected to be in the on-state, which is reminiscent of the aberrant behavior of Padron 2.1 described in section 3.4.2. In this context, it is important to mention that reproducibility of absorption spectra of Padron 2.1 was problematic. Therefore, figure $3.16 \mathrm{e}$ shows only a selected spectrum.
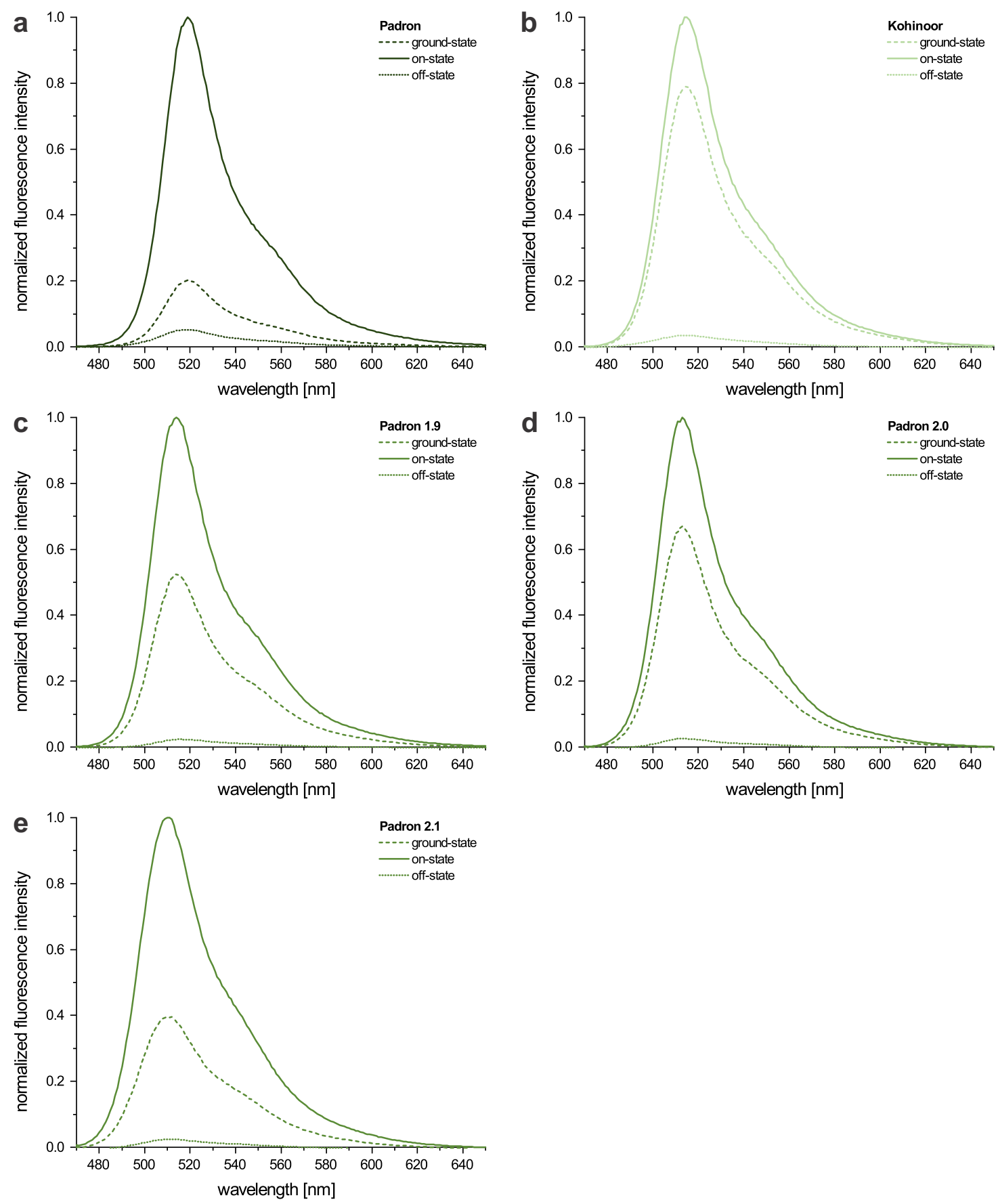

Figure 3.17: Emission spectra of switching states. Proteins were switched in a cuvette and emission spectra were recorded with an excitation wavelength of $460 \mathrm{~nm}$ for (a) Padron, (b) Kohinoor, (c) Padron 1.9, (d) Padron 2.0, and (e) Padron 2.1. Emission spectra were normalized to the on-state maximum. 
Along with the absorption spectra described above, fluorescence emission spectra of the switching states were recorded, figure 3.17 depicts the resulting data. Similar to the absorption spectra, the emission spectra tendentiously represented switching states described in section 3.4.2. Compared to Padron, which had an emission peak at $519 \mathrm{~nm}$, new Padron variants and Kohinoor emission peaks were blue-shifted by $5-7 \mathrm{~nm}$ (tab. 3.6).

For the equilibrium ground-state, excitation spectra and additional emission spectra were generated during the analysis of $\mathrm{pH}$ dependency, which is described in the following section 3.4.3.2. Excitation and emission spectra for $\mathrm{pH} 7.5$ from these measurements are depicted in figure 3.18 and associated peak data are listed in table 3.6. The observed blue-shift of emission peaks described above is also shown by this data. Minor differences in peak values exist but are most likely to be attributed to differing methods and instruments as well as the fact that emission peaks of ground- and on-state are compared at this point. Excitation spectra of new variants were blue-shifted compared to Padron by $4-9 \mathrm{~nm}$.

After all, the mutations introduced to the new Padron variants resulted in a hypsochromic shift of absorption, excitation and emission spectra, albeit to a different extent. This change occurred along with altered protonation states of the on-state, which was shifted towards the protonated chromophore for these variants.

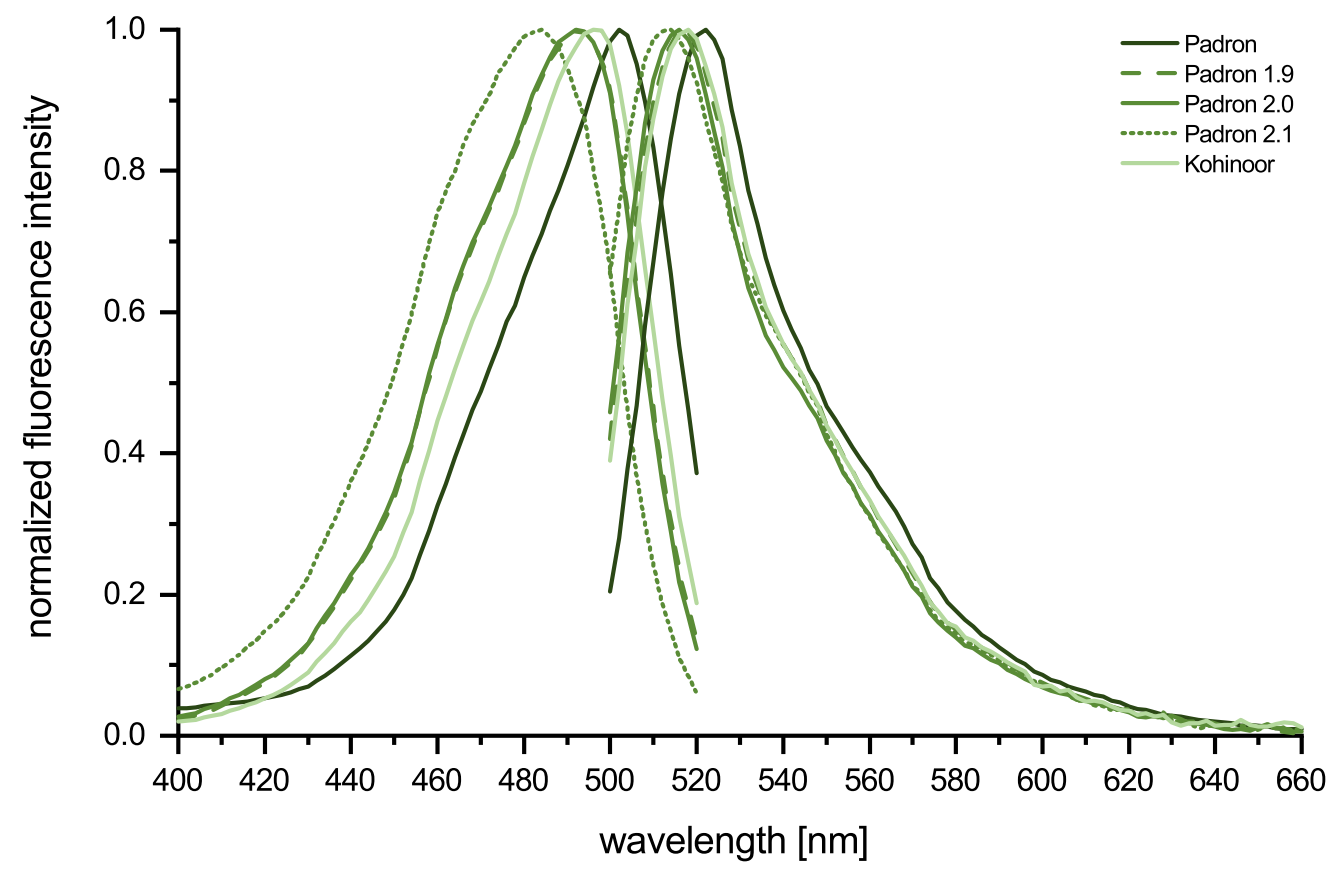

Figure 3.18: Excitation and emission spectra of the equilibrium ground-state. Excitation and emission spectra were recorded at $\mathrm{pH} 7.5$ with detection at $550 \mathrm{~nm}$ or excitation at $470 \mathrm{~nm}$, respectively.

\subsubsection{2 $\mathrm{pH}$ dependent absorption spectra}

The spectral analysis described above was conducted at $\mathrm{pH}$ 7.5. This approach allows for comparison of different proteins under standardized conditions, but is incomplete with re- 


\section{Results}

gards to live-cell applications, where the $\mathrm{pH}$ values vary over a larger range. Padron and new variants were thus characterized at different $\mathrm{pH}$ values. For this analysis, absorption, excitation, and emission spectra were recorded for $\mathrm{pH}$ values from 3 to 10.5 (cf. methods sec. 5.5.9).
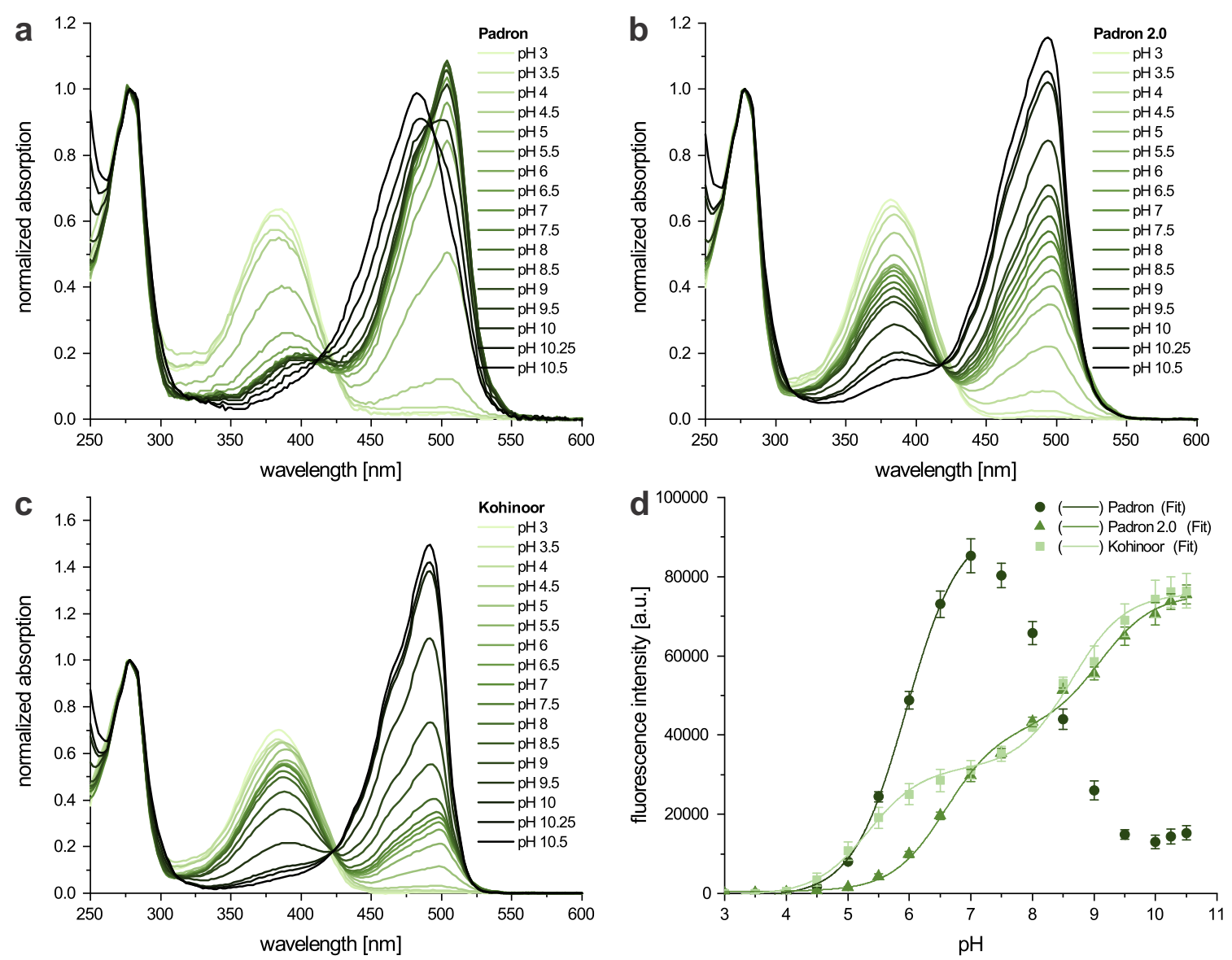

Figure 3.19: pH dependent absorption and fluorescence emission. Equilibrium ground-state absorption spectra were measured over a range of $\mathrm{pH}$ values for (a) Padron, (b) Padron 2.0, and (c) Kohinoor. (d) Fluorescence emission from the same samples was measured with a 485/20 excitation and a 528/20 detection filter and results are depicted as a function of $\mathrm{pH}$. Detector sensitivity was scaled to the brightest wells of each sample.

With increasing $\mathrm{pH}$, Padron 2.0 and Kohinoor showed a monotonous decline of the protonated peak with concurrent emergence of the deprotonated peak (fig. 3.19b, c), albeit at different pH dependencies. Absorption spectra of Padron displayed a similar response to increasing $\mathrm{pH}$ at low $\mathrm{pH}$ values but an opposing one at $\mathrm{pH}$ values above 7 (fig. 3.19a) with aberrantly-shaped peaks above $\mathrm{pH} 10$, suggesting declining fluorescence emission in response to $488 \mathrm{~nm}$ excitation at high $\mathrm{pH}$. This is supported by the fluorescence emission data shown in figure 3.19c, which shows fluorescence intensity measured at different $\mathrm{pH}$ values. While emitted fluorescence increased with the $\mathrm{pH}$ for Padron 2.0 and Kohinoor as expected based on their absorption spectra, emitted fluorescence peaked for Padron at $\mathrm{pH} 7$ and declined at higher $\mathrm{pH}$ values. At $\mathrm{pH}$ values where absorption spectra displayed altered peak shapes for Padron, measured 
emitted fluorescence slightly increased. Interestingly, the position of the deprotonated peak shifted for the new variants with changing $\mathrm{pH}$ values (fig. 7.4d). $\mathrm{pH}$ dependency of Padron 1.9 and Padron 2.1 was characterized accordingly, results are shown in the appendix in figure 7.4. In short, Padron 1.9 displayed higher relative fluorescence intensities dependent on the $\mathrm{pH}$ values, while Padron 2.1 displayed lower brightness than Padron 2.0 at low values but higher at pH 8 and above. However, this data only allows for comparison of the different pH responses. Absolute fluorescence intensities cannot be compared as the detector sensitivity was scaled to the brightest signal in each experiment. Full $\mathrm{pH}$ dependent excitation and emission spectra are depicted in the appendix in figures 7.5 and 7.6.

$\mathrm{pH}$ dependent fluorescence intensity was used for calculation of $\mathrm{p} K_{a}$ values shown in table 3.7. All new Padron variants and Kohinoor had $2 \mathrm{p} K_{a}$ values, suggesting multiple protonation equilibria. In contrast, Padron had only one $\mathrm{p} K_{a}$ value due to its decline of fluorescence intensity at high $\mathrm{pH}$. While the lower $\mathrm{p} K_{a 1}$ of 5.3 of Kohinoor was below the one of Padron (5.9), $\mathrm{p} K_{a 1}$ values of the new Padron variants were in the range of 6.3-6.6 and thus higher compared to Padron. This suggested an improved applicability of Kohinoor at lower $\mathrm{pH}$ values and an impaired performance of the new variants with respect to fluorescence intensities. However, both the new Padron variants and Kohinoor showed similar $\mathrm{p} K_{a 2}$ values in the range of 8.6-9.1. As these variants showed no decline of fluorescence intensity or emergence of aberrant absorption spectra, they were expected to show higher fluorescence intensity and greater stability at high $\mathrm{pH}$ values.

Table 3.7: $\mathrm{p} K_{a}$ values measured $(\mathrm{n}=2-3)$

\begin{tabular}{lcc} 
Protein & $\mathbf{p} \boldsymbol{K}_{a 1}$ & $\mathbf{p} \boldsymbol{K}_{a 2}$ \\
\hline Padron & $5.9 \pm 0.0$ & n.a. \\
Padron 1.9 & $6.3 \pm 0.1$ & $8.8 \pm 0.1$ \\
Padron 2.0 & $6.6 \pm 0.0$ & $9.1 \pm 0.0$ \\
Padron 2.1 & $6.3 \pm 0.1$ & $8.3 \pm 0.0$ \\
Kohinoor* & $5.3 \pm 0.1$ & $8.6 \pm 0.1$ \\
\hline \multicolumn{2}{c}{ "published values: 5.9 and $8.6[121]^{2}$}
\end{tabular}

\subsubsection{Extinction coefficient, quantum yield and fluorescence lifetime}

Aside from the excitation and emission spectra shown above, the extinction coefficient, quantum yield, and fluorescence lifetime are characteristics of a fluorescent protein influencing their performance in fluorescence microscopy. These parameters were determined for Padron and Kohinoor as well as the new variants and are described below.

On-state absorption spectra depicted in figure 3.16 and on-state emission spectra shown in figure 3.17 were used for calculation of the extinction coefficient and quantum yield. Both were calculated based on comparative measurements: the former based on absorption spectra of mEGFP [171] (cf. methods sec. 5.5.5), the latter relative to Padron [120] (cf. methods sec. 5.5.6). Fluorescence lifetime was measured absolutely (cf. methods sec. 5.5.8). An overview of the results is given in table 3.8 . 


\section{Results}

The changed ratio of absorption peaks described in section 3.4.3.1 resulted in profoundly lower extinction coefficients of new Padron variants for the absorption peak maximum of the fluorescent deprotonated state. At pH 7.5, Padron 1.9 displayed the highest extinction coefficient of the new variants at $24,005 \pm 670 \mathrm{M}^{-1} \mathrm{~cm}^{-1}$, which was still $40 \%$ lower than the extinction coefficient of Padron at $39,938 \pm 1,489 \mathrm{M}^{-1} \mathrm{~cm}^{-1}$. Padron 2.0, 2.1, and Kohinoor displayed a further reduction of this value to approximately $30-40 \%(16,167 \pm 585$, $15,747 \pm 5,091$, and $12,588 \pm 410 \mathrm{M}^{-1} \mathrm{~cm}^{-1}$, respectively).

The quantum yield of Kohinoor of 0.73 was higher than the quantum yield of Padron of 0.64 , while Padron 1.9 featured a similar quantum yield (0.60). This value was further reduced to 0.49 in Padron 2.0 and 0.28 in Padron 2.1, which was less than $50 \%$ of the quantum yield of Padron.

Because brightness of RSFPs is one of the critical features in practical applications, the extinction coefficient and quantum yield were used to calculate the molecular brightness (tab. 3.8). Both Padron 2.0 and Kohinoor featured a brightness of about one third of Padron, which has a molecular brightness of 25.6, and displayed considerably lower values of 7.9 and 9.2, respectively. Padron 1.9 with a molecular brightness of 14.4 was approximately $50 \%$ brighter than the former variants while the brightness of Padron 2.1 was about $50 \%$ lower with a value of 4.4 .

With respect to fluorescence lifetime, Padron and Kohinoor featured similar lifetimes of 3.4 and $3.5 \mathrm{~ns}$ that were only marginally reduced to 3.1 and $3.0 \mathrm{~ns}$ in Padron 1.9 and 2.0, respectively. In contrast, this quantity was reduced in Padron 2.1 by about $35 \%$ to $2.2 \mathrm{~ns}$.

Table 3.8: Extinction coefficients, quantum yields and fluorescence lifetimes of Padron variants

\begin{tabular}{|c|c|c|c|c|}
\hline Protein & $\begin{array}{c}\text { Extinction } \\
\text { coefficient } \\
{\left[\mathrm{M}^{-1} \mathbf{c m}^{-1}\right]}\end{array}$ & $\begin{array}{l}\text { Quantum } \\
\text { yield }\end{array}$ & $\begin{array}{l}\text { Molecular } \\
\text { brightness }\end{array}$ & $\begin{array}{c}\text { Fluorescence } \\
\text { lifetime } \\
{[\mathrm{ns}]}\end{array}$ \\
\hline & \multicolumn{3}{|c|}{$\mathrm{pH} 7.5$, on-state maximum } & $\mathrm{pH} 7.5$, ground-state \\
\hline Padron & $39,938 \pm 1,489^{a}$ & $0.64^{b}$ & 25.6 & $3.4 \pm 0.02$ \\
\hline Padron 1.9 & $24,005 \pm 670$ & $0.60 \pm 0.01$ & 14.4 & $3.1 \pm 0.03$ \\
\hline Padron 2.0 & $16,167 \pm 585$ & $0.49 \pm 0.02$ & 7.9 & $3.0 \pm 0.05$ \\
\hline Padron 2.1 & $15,747 \pm 5,091$ & $0.28 \pm 0.02$ & 4.4 & $2.2 \pm 0.01$ \\
\hline Kohinoor & $12,588 \pm 410^{c}$ & $0.73 \pm 0.03^{d}$ & 9.2 & $3.5 \pm 0.09$ \\
\hline
\end{tabular}

\subsubsection{Tagging and oligomerization}

The new Padron variants were a considerable improvement compared to both Padron and Kohinoor with regards to the switching capabilities. While some parameters, such as tolerance of low pH or molecular brightness, were better in the original Padron, both switching to 
the off-state and switching-fatigue were significantly improved. Resistance to photobleaching and switching to the on-state were improved as well, but to a lesser extent. Most importantly, the new variants allowed for suppression of apparent fluorescence under simultaneous irradiation with light of 405 and $488 \mathrm{~nm}$, which was not feasible with the original Padron or Kohinoor. These enhancements were expected to greatly improve the performance in RESOLFT nanoscopy, which has not been demonstrated with Padron so far. However, livecell applications require labeling of target structures without interfering with their biological function. To address the capabilities of new Padron variants in this respect the oligomerization tendency was analyzed.

\subsubsection{In vitro oligomerization tendencies}

For an initial estimation of oligomerization tendencies, proteins were subjected to seminative polyacrylamide gel electrophoresis (PAGE) (cf. methods sec. 5.4.5.2). Due to non-denaturing sample handling prior to electrophoresis, proteins maintained their secondary structure. This method reveals stable interaction of fluorescent proteins in multimeric states but is expected to be insensitive to weak interactions due to the sodium dodecylsulfate (SDS) content of gel and buffer, which is assumed to attenuate weak interactions. DsRed [94], dTomato [98] and mEGFP [171] were used as markers of the oligomeric state. As GFP-like proteins with a $\beta$-barrel structure they have a size similar to Padron and are suitable as markers for tetrameric [172], dimeric [98] and monomeric [171] oligomerization. Figure 3.20 shows fluorescence emission of proteins after electrophoresis (a) as well as total protein content of the gel (b).
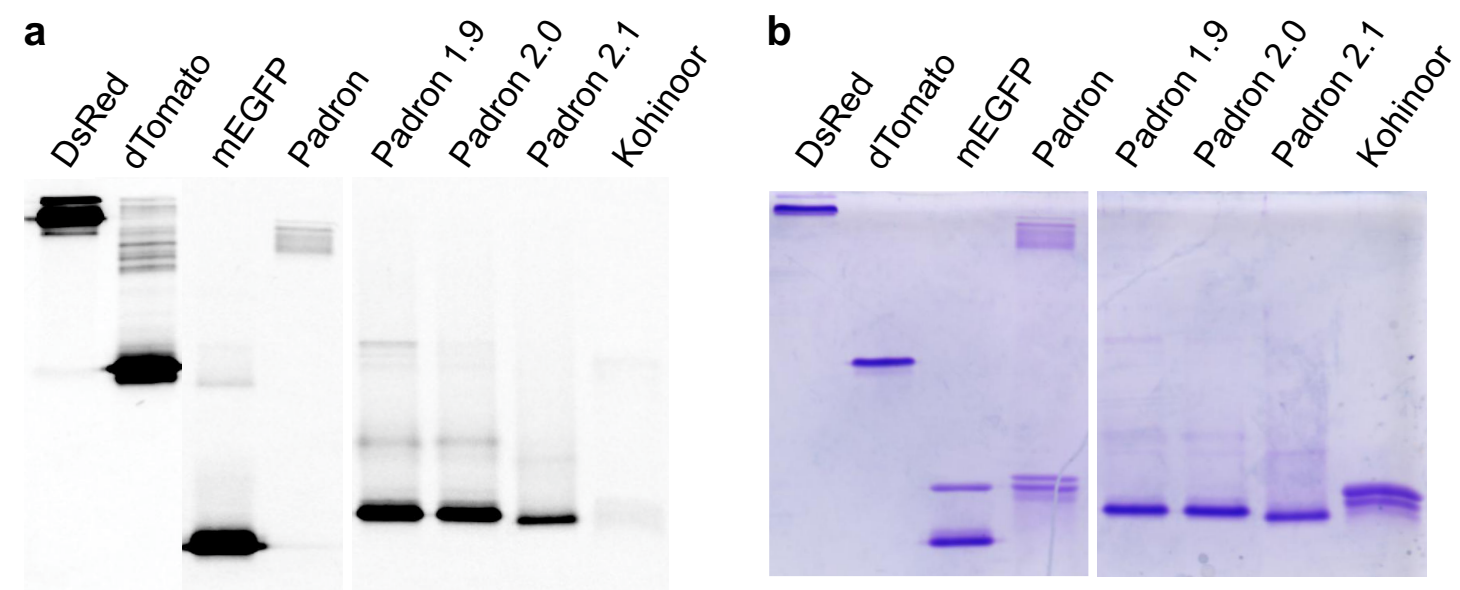

Figure 3.20: Seminative polyacrylamide gel electrophoresis. Proteins were analyzed with a seminative PAGE with non-denaturing sample handling prior to application to the gel. DsRed, dTomato, and mEGFP were added as oligomeric state markers for tetrameric, dimeric, and monomeric fluorescent proteins, respectively. (a) Fluorescence image of the gel in the red (DsRed, dTomato) and green (others) channel. (b) Coomassie staining of the same gel. 


\section{Results}

The original Padron was published as a monomer at $37^{\circ} \mathrm{C}$ with a slight tendency to dimerize at $4^{\circ} \mathrm{C}[120]$. In the seminative gel Padron appeared in two distinct populations (monomeric and tetrameric) of which only the latter one maintained fluorescence emission after electrophoresis. Kohinoor appeared as a monomer as published [121], but lost nearly all fluorescence under these conditions. Conversely, all new variants presented in this thesis showed monomeric behavior in seminative PAGE and maintained their fluorescence. All Padron variants passed the gel slower than mEGFP, although their molecular weight was very similar $(29.4 / 30.7 \mathrm{kDa}$ for Padrons with and without GFPends, $30.9 \mathrm{kDa}$ for mEGFP; molecular weights include additional amino acids originating from the $6 x$ His-tag for purification). Thus, the difference was most likely to be attributed to different secondary structures.

The loss of fluorescence of Padron and Kohinoor suggests susceptibility to the SDS as a denaturing agent in the gel and implies lower stability of these two proteins compared to the new variants. The latter are possibly less sensitive to denaturing conditions due to the addition of GFPends: The fluorescence of Kohinoor in this experiment could be restored by the addition of GFPends (fig. 7.7).

\subsubsection{Size exclusion chromatography}

While seminative PAGE allows for a fast and good estimate of oligomeric tendencies, weak associations between monomers are lost due to the denaturing properties of SDS. Purified protein samples were therefore analyzed via size exclusion chromatography (SEC). Measurements were run at $6^{\circ} \mathrm{C}$, which was expected to reveal even weak interaction of monomers due to the tendency of the original Padron to dimerize at lower temperatures [120]. Consequently, minor tendencies of RSFPs to form oligomers at this temperature were expected to be attenuated at higher temperatures employed for live-cell applications.

Figure 3.21 shows the results of SEC measurements. Oligomeric markers were clearly distinguishable, and Padron displayed the same behavior as in seminative PAGE with two distinct fractions. The larger one behaved as a monomer, while a smaller but considerable fraction displayed tetrameric behavior. The existence of both fractions suggested two populations that were stable over the course of the experiment. Padron 2.1 results were similar to Padron, but the larger fraction with an elution volume distinctively smaller than that of DsRed suggested stable octamers. Another smaller peak supported this notion of strong interaction of Padron 2.1 monomers. Padron 1.9 and 2.0 on the other hand showed only a single monomeric peak with a small dimeric peak for Padron 1.9, which amounted to a negligible fraction. Kohinoor also displayed a single elution peak that was positioned between the monomeric and dimeric marker. In contrast to the published data, which show Kohinoor as a monomer, this suggested a fast transient interaction of Kohinoor monomers. Fast rate constants for association of monomers and subsequent dissociation would render monomers and dimers inseparable for SEC [173].

After all, Padron 2.0 was least inclined to oligomerize based on the data presented. Although higher temperatures in biological samples can lower or attenuate interactions between pro- 
teins, as it was shown for Padron and Kohinoor [120, 121], locally increased concentrations in labeled structures have to be kept in mind that could potentially shift the equilibrium to oligomerized RSFPs, even at $37^{\circ} \mathrm{C}$. This was already noticeable at $6{ }^{\circ} \mathrm{C}$ : If Padron variants were not equilibrated after dilution to $10 \mu \mathrm{M}$, the ratio of monomeric and tetrameric peaks was shifted towards the oligomer so that all variants displayed oligomerization (fig. 7.8).
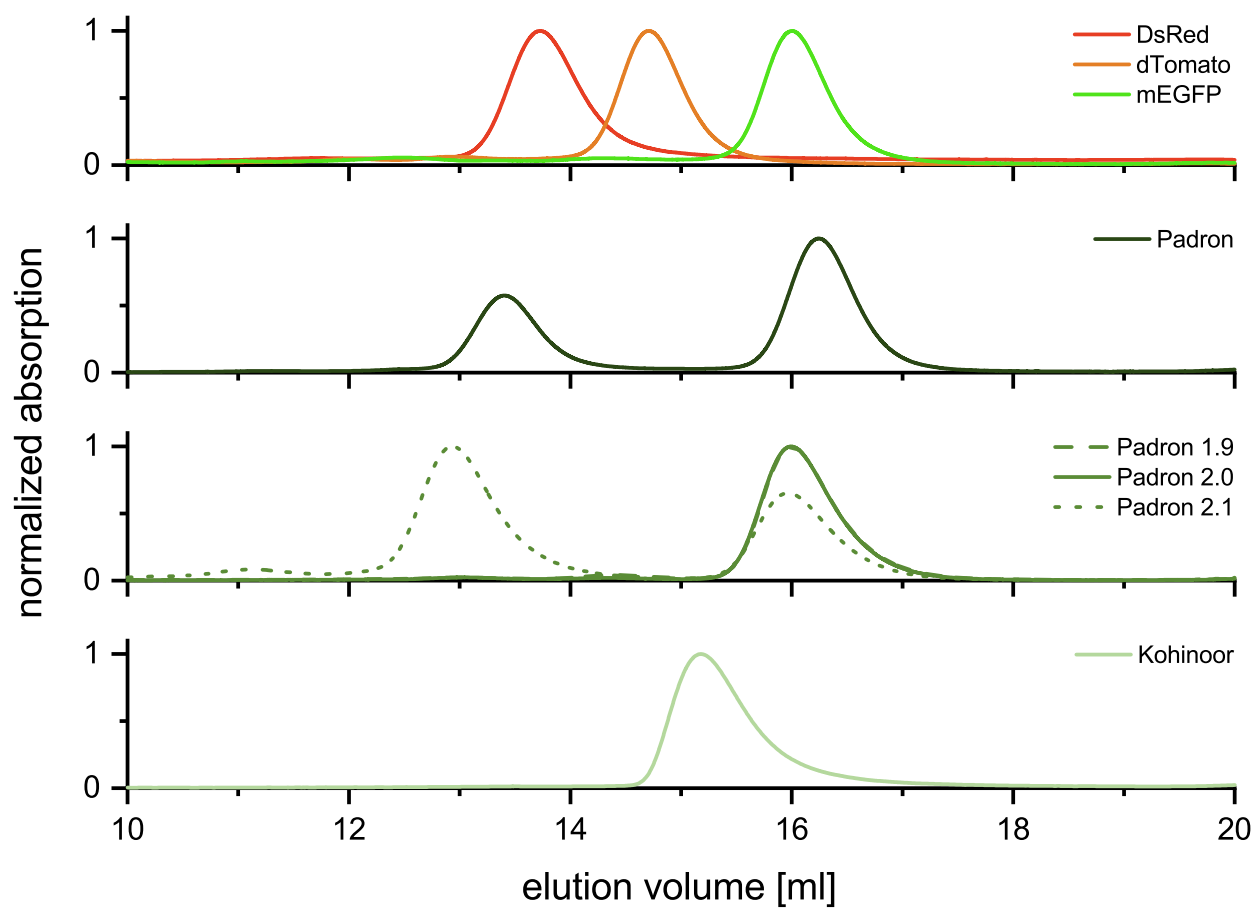

Figure 3.21: Size exclusion chromatography. Protein absorption was detected after size exclusion chromatography (SEC) of protein samples with $10 \mu \mathrm{M}$ concentration. SEC was performed at $6^{\circ} \mathrm{C}$, and DsRed, dTomato and mEGFP were used as oligomeric markers. Padron 1.9 overlaps with Padron 2.0 for the most part. Protein absorption was measured at $280 \mathrm{~nm}$.

\subsubsection{Labeling of cellular structures with Padron variants}

Padron 2.0 displayed the lowest tendency to oligomerize among the variants analyzed (sec. 3.4.4) and was therefore expected to perform well as a tag. To test the suitability of all Padron variants for biological applications a multitude of cellular structures was labeled and imaged with diffraction-limited confocal microscopy of transiently transfected HeLa cells (cf. methods sec. 5.1.2). Figure 3.22 depicts 14 different structures or compartments for Padron, Kohinoor and the new Padron variants. For all proteins, cells with aberrant shape or aggregated structures were visible, which was assumed to be caused by strong overexpression. The figure depicts selected cells with adequate expression levels.

In general, all cells with Padron labeled structures displayed low fluorescence intensities compared to the other proteins which was expected to be due to the impaired ability of Padron to mature at $37^{\circ} \mathrm{C}$ (sec. 3.3). All other variants analyzed showed fluorescence intensities resembling their previous characterization. 

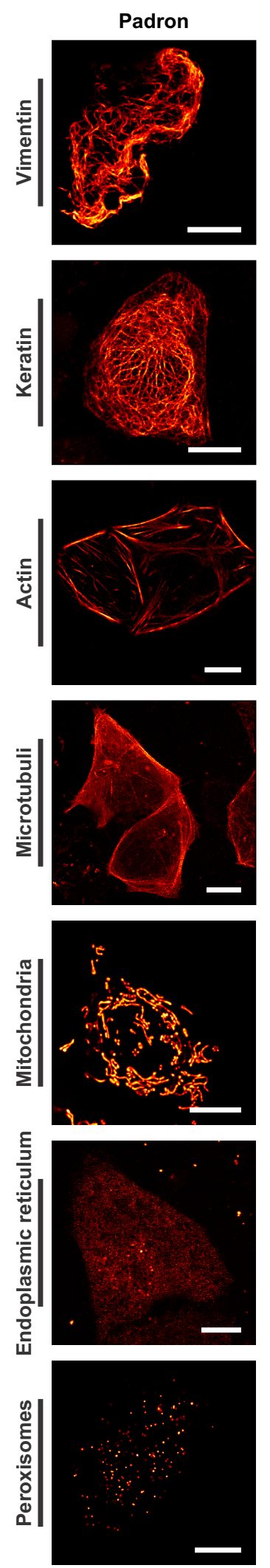

Padron 1.9

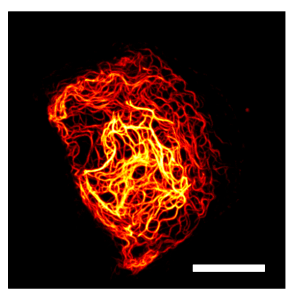

Padron 2.0
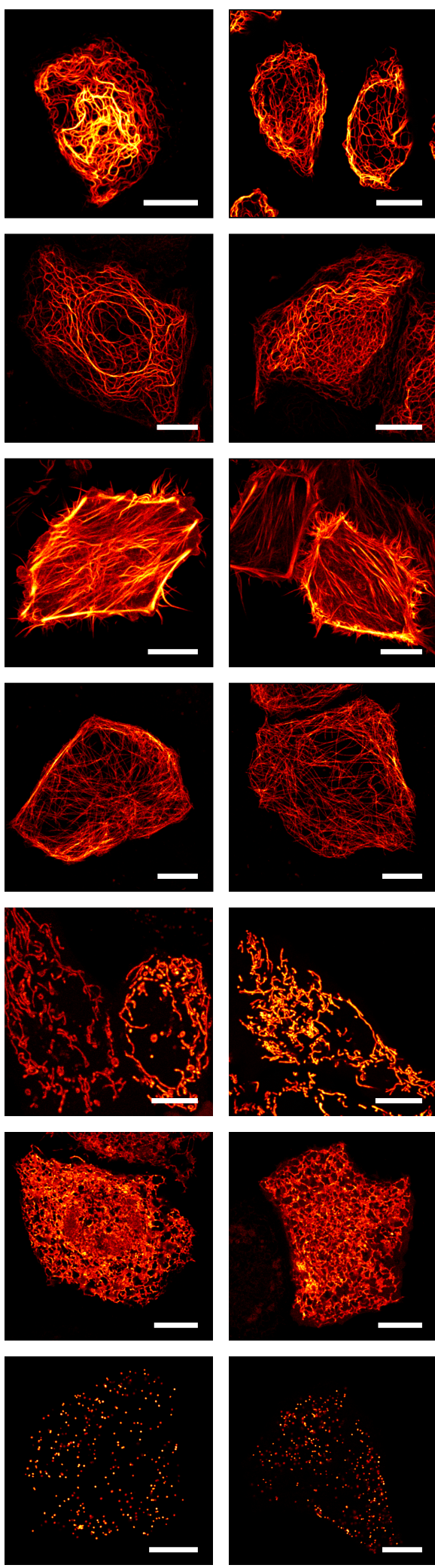

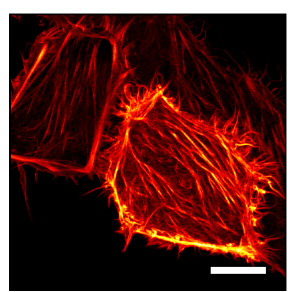

Padron 2.1
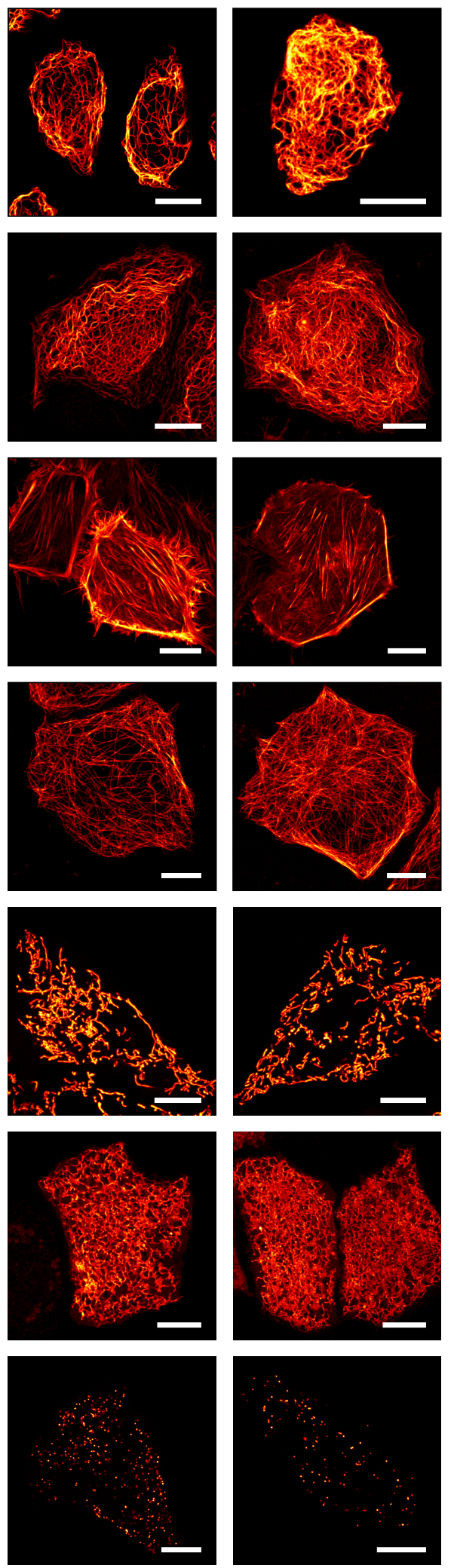

Kohinoor
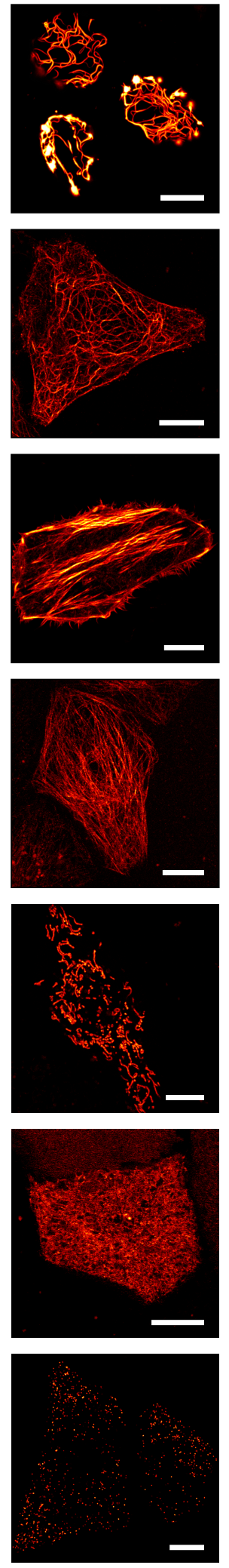

Figure 3.22: Labeling of cellular structures with Padron variants. Figure description can be found on page 60 . 

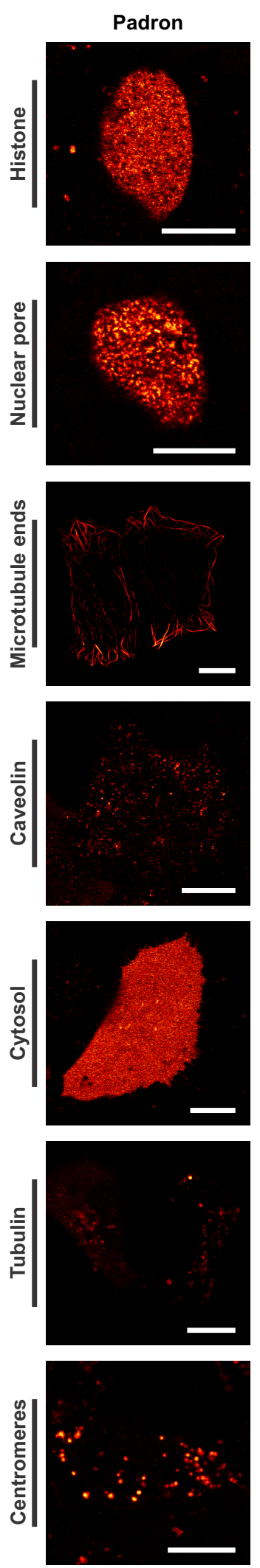

Padron 1.9

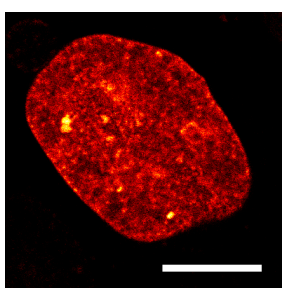

Padron 2.0
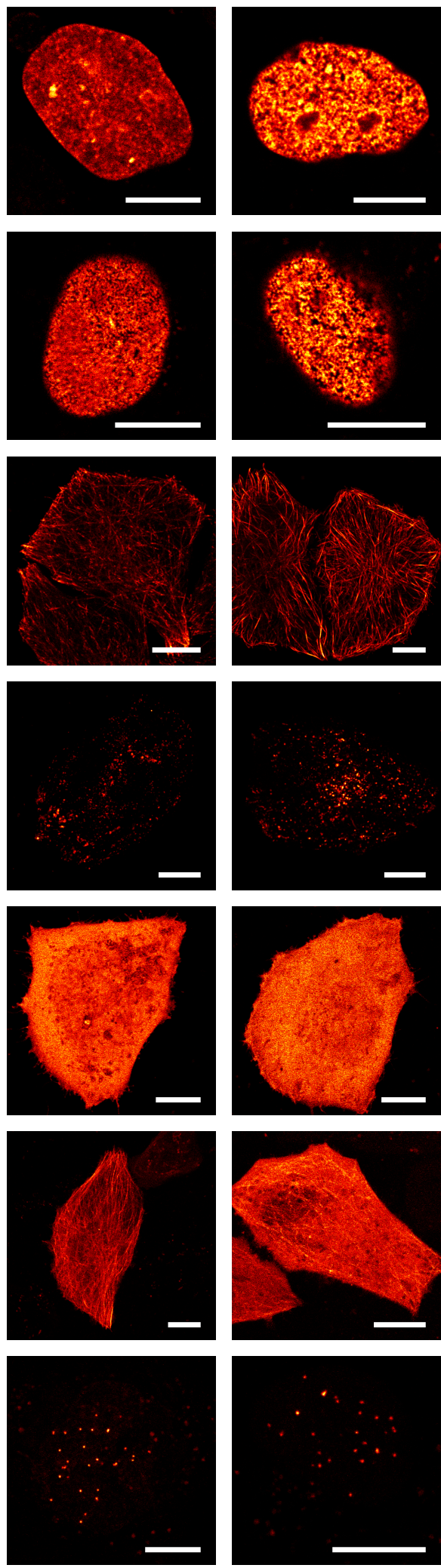

Padron 2.1
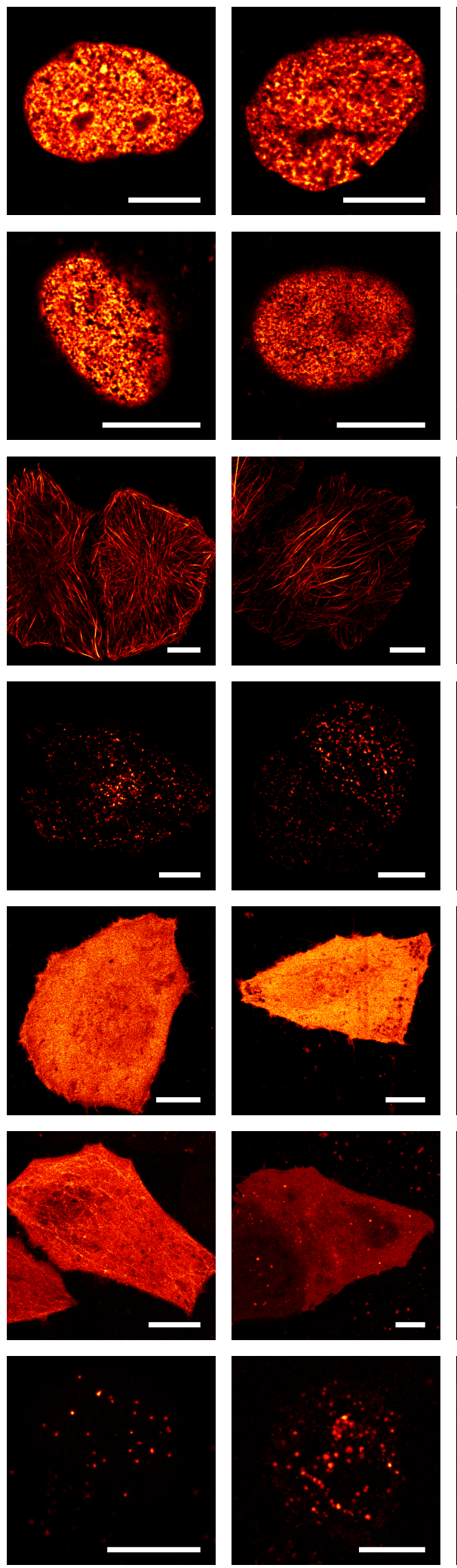

Kohinoor
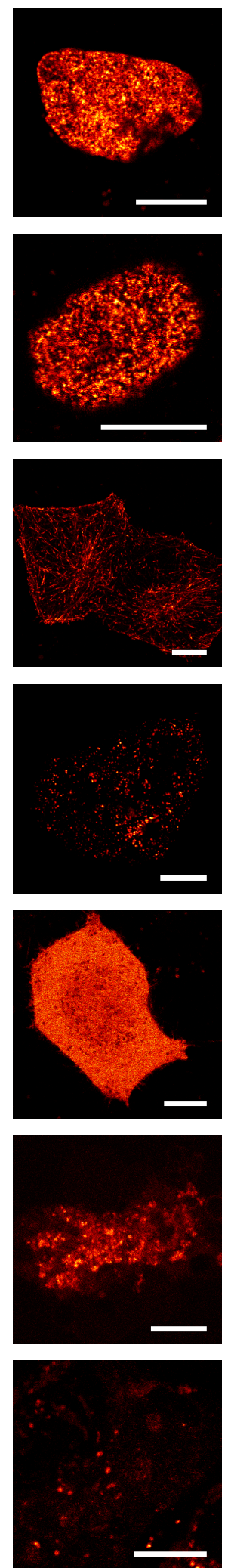

Figure 3.22 (cont.): Labeling of cellular structures with Padron variants. Figure description can be found on page 60 . 
Figure 3.22 (cont.): Labeling of cellular structures with Padron variants. HeLa cells were transiently transfected with the plasmids described in section 5.1.2.1. Fluorescent proteins were either fused to target structures or to localization sequences. All images were recorded in confocal mode as single plane or as z-stack. Vimentin: Vim-FP, z-projection. Keratin: cytokeratin18-FP, z-projection. Lifeact: lifeact-FP, z-projection. Microtubules: FP-Map2, z-projection. Mitochondria: TS-FP, z-projection. Endoplasmic reticulum: TS-FP, single plane. Peroxisomes: FP-TS, single plane. Histone: FP-H2bn, single plane. Nuclear pore complex: FP-Nup50, single plane. Microtubule ends: EB3-FP, z-projection. Caveolin: caveolin1-FP, single plane. Cytosol: FP, single plane. Tubulin: FP-tubulin. Centromeres: FP-CenPC1, single plane. FP, fluorescent protein. TS, targeting sequence. Color maps are different for every image and allow for no comparison. Scale bars: $10 \mu \mathrm{m}$.

Vimentin had a tendency to build aggregated filaments after labeling. This issue was moderate for the new Padron variants but rather pronounced for Kohinoor, where vimentin was tightly wrapped around the nucleus displaying aggregation artifacts. Padron also tended towards aggregation. Keratin structures were as expected for all proteins. The same was true for indirect labeling of actin and microtubuli with lifeact and map2, respectively. However, Padron showed less dense labeling of actin and failed to properly label microtubuli with map2 fusion constructs. Direct labeling of microtubuli with overexpressed tubulin fusion constructs was problematic for all Padron variants. While Padron 1.9 and 2.0 fusion constructs resulted in labeled microtubuli that were masked by cytosolic fluorescence, no structures were visible for Padron 2.1, which in contrast tended towards building aggregates. Padron and Kohinoor failed entirely in labeling microtubuli as tubulin fusion constructs. EB3 was used to mark microtubule ends in HeLa cells, which could be facilitated by Padron 1.9 and Kohinoor. Padron 2.0 and 2.1 fusion constructs bound to the entirety of microtubuli, which was expected for strong overexpression of EB3. EB3-Padron constructs resulted in filamentous structures that were predominantly located to the perimeter of the cell. While protein import into the mitochondrial matrix was unproblematic and no fragmentation of mitochondria was visible, localization to the endoplasmic reticulum (ER) was facilitated only by the new variants and Kohinoor. Padron on the other hand failed to properly label the ER. In contrast, peroxisomal localization and labeling of histone $\mathrm{H} 2 \mathrm{bn}$ and nucleoporin was feasible for all variants including Padron. Caveolin and centromere labeling displayed no aberrant behavior for all proteins, and cytosolic expression of Padron variants was free of aggregates.

The new Padron variants proved to be suitable for biological applications, the targeted structures could be imaged with proper labeling. In addition, preliminary data showed that endogenous overexpression of tubulin labeled with Padron0.9, a Padron variant with an even increased tendency for oligomerization [140], was feasible in Drosophila melanogaster (fig. 7.9 in the appendix). While Kohinoor was on par for many structures or compartments, fusion of Kohinoor to vimentin and tubulin resulted in impaired labeling compared to Padron 1.9 and 2.0. Padron 2.1 could target structures better than it was expected based on its tendency to oligomerize (fig. 3.4.4.2), suggesting that low fluorescence and artificial structures observed with the original Padron mainly resulted from incomplete maturation. Consequently, im- 
proved maturation and expression of Padron 2.0 along with its low tendency to oligomerize rendered it best suitable for live-cell applications.

\subsection{Super-resolution microscopy with Padron 2.0}

After the new Padron variants had been established for tagging of cellular structures, transiently transfected HeLa cells with labeled vimentin were chosen as a sample to establish RESOLFT microscopy with Padron 2.0. This variant of Padron was chosen as it had proven to be most versatile in the majority of characteristics that are important for RESOLFT imaging. Vimentin is often used as model structure for RESOLFT nanoscopy due to its abundance of filamentous structures [124, 174, 175]. To quantify resolution improvements, line profiles were measured across filaments and subsequently fitted with a Lorentzian fit. The full-width half-maximum values of the fits were compared.

\subsubsection{Evaluation of vimentin-Padron 2.0 structures with STED nanoscopy}

Prior to RESOLFT microscopy, vimentin structures were imaged at the same microscope with STED nanoscopy to estimate achievable resolution (cf. methods sec. 5.6.5). Figure 3.23 shows the resulting image with an overlay of the confocal overview image. Of the 10 line profiles measured in figure 3.23, the lowest FWHM was $48.8 \mathrm{~nm}$, which exhausted the expected achievable resolution gain with the applied pixel size of $25 \mathrm{~nm}$.

In general, filament FWHM values could easily achieve a width of around $65 \mathrm{~nm}$, which is approximately $50 \%$ wider than the FWHM values of around $40 \mathrm{~nm}$ published by Danzl et al. for protected STED microscopy of vimentin-rsEGFP2 [174]. The difference of FWHM presented here most likely is based on a lower sampling rate and the observed tendency for condensation of vimentin filaments. In addition, the microscope was not built for STED nanoscopy but rather optimized for RESOLFT applications. The STED doughnut was thus less optimized than the $405 \mathrm{~nm}$ RESOLFT doughnut and not perfectly circular due to optical aberration (cf. fig. 5.3 in methods sec. 5.6.3), which likely had an influence on the resulting STED images. 

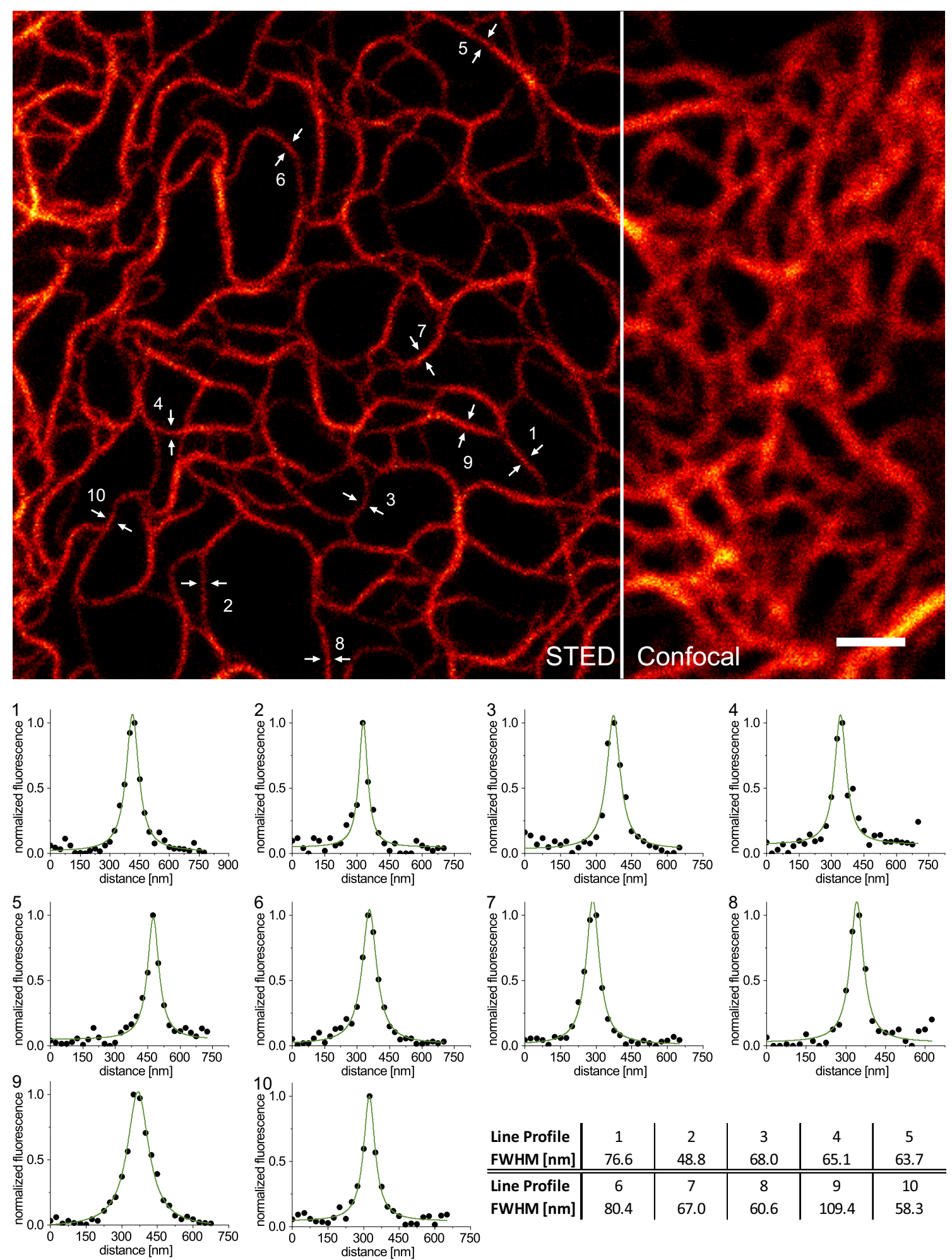

\begin{tabular}{l|c|c|c|c|c} 
Line Profile & 1 & 2 & 3 & 4 & 5 \\
FWHM [nm] & 76.6 & 48.8 & 68.0 & 65.1 & 63.7 \\
\hline \hline Line Profile & 6 & 7 & 8 & 9 & 10 \\
FWHM [nm] & 80.4 & 67.0 & 60.6 & 109.4 & 58.3
\end{tabular}

Figure 3.23: STED image of vimentin-Padron 2.0 fusion constructs in transiently transfected HeLa cells. HeLa cells were imaged with $25 \mathrm{~nm}$ pixel size in line step STED and confocal mode. Line profiles were measured at 10 manually selected positions with 3 adjacent lines and modeled with a Lorentzian fit. FWHM of the fits are listed in the table. Scale bar: $1 \mu \mathrm{m}$. 


\subsubsection{RESOLFT microscopy}

As vimentin-Padron 2.0 fusion constructs were a suitable sample for super-resolution approaches, RESOLFT microscopy was established using this sample in the next step. Initially, images were recorded with a sequential switching approach as illustrated in figure 3.24. Images were recorded in pixel step mode performing activation, doughnut-based off switching, and fluorescence readout at every scan position in the sample.

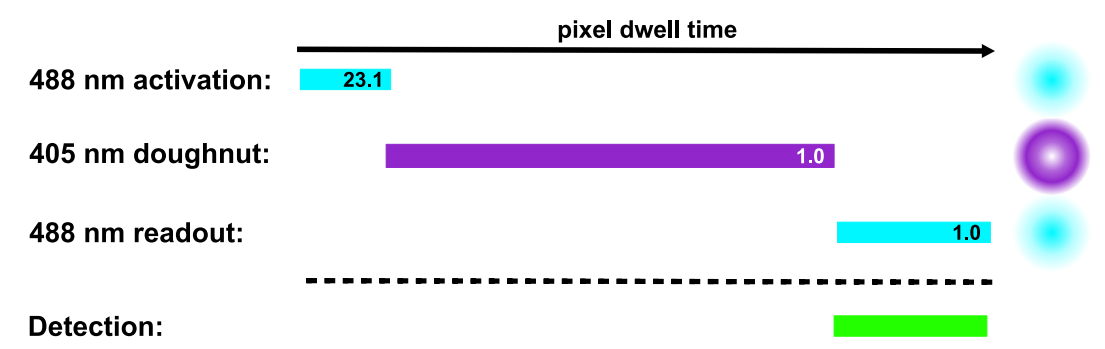

Figure 3.24: RESOLFT microscopy scheme with sequential switching sequence. A RESOLFT image recorded with the illustrated laser sequence is shown in figure 3.25. Numbers are laser intensities in $\mathrm{kW} / \mathrm{cm}^{2}$.

For this purpose, Padron 2.0 fluorophores were switched to the on-state with a $488 \mathrm{~nm}$ laser at the start of each pixel to ensure maximal fluorescence detection at every coordinate. From this state, fluorophores were switched to the off-state with a $405 \mathrm{~nm}$ doughnut-shaped beam rendering peripheral proteins non-fluorescent while central ones remained fluorescent. Latter RSFPs then contributed to fluorescence detection during the final readout step with a Gaussian-shaped $488 \mathrm{~nm}$ laser beam featuring a lower intensity to prevent peripheral fluorophores from returning to the on-state.

A resulting RESOLFT image is shown in figure 3.25 displaying improved resolution and a good signal to background ratio. FWHM of analyzed filaments was as low as $\sim 58 \mathrm{~nm}$ with multiple other filaments supporting this data. The image shown measures 568x431 pixels $(14.2 \times 10.8 \mu \mathrm{m})$ with a dwell time of $540 \mu \mathrm{s}$ each and a total imaging time of $132 \mathrm{~s}$. An additional image recorded with the sequential switching approach is is shown in figure 7.10 in the appendix.

However, this mode of RESOLFT imaging required repeated cycling of fluorophores in line accumulation mode (that is, measuring every pixel multiple times pixel line wise with the same switching scheme) to image structures significantly dimmer than vimentin as the readout step could not be prolonged infinitely. With long readout dwell times or higher readout intensities, peripheral fluorophores would switch back to the on-state and attenuate the resolution improvement. On the other hand, increasing fluorescence readout with, for example, line accumulation would multiply the switching cycles performed by every fluorophore, increasing switching fatigue. 

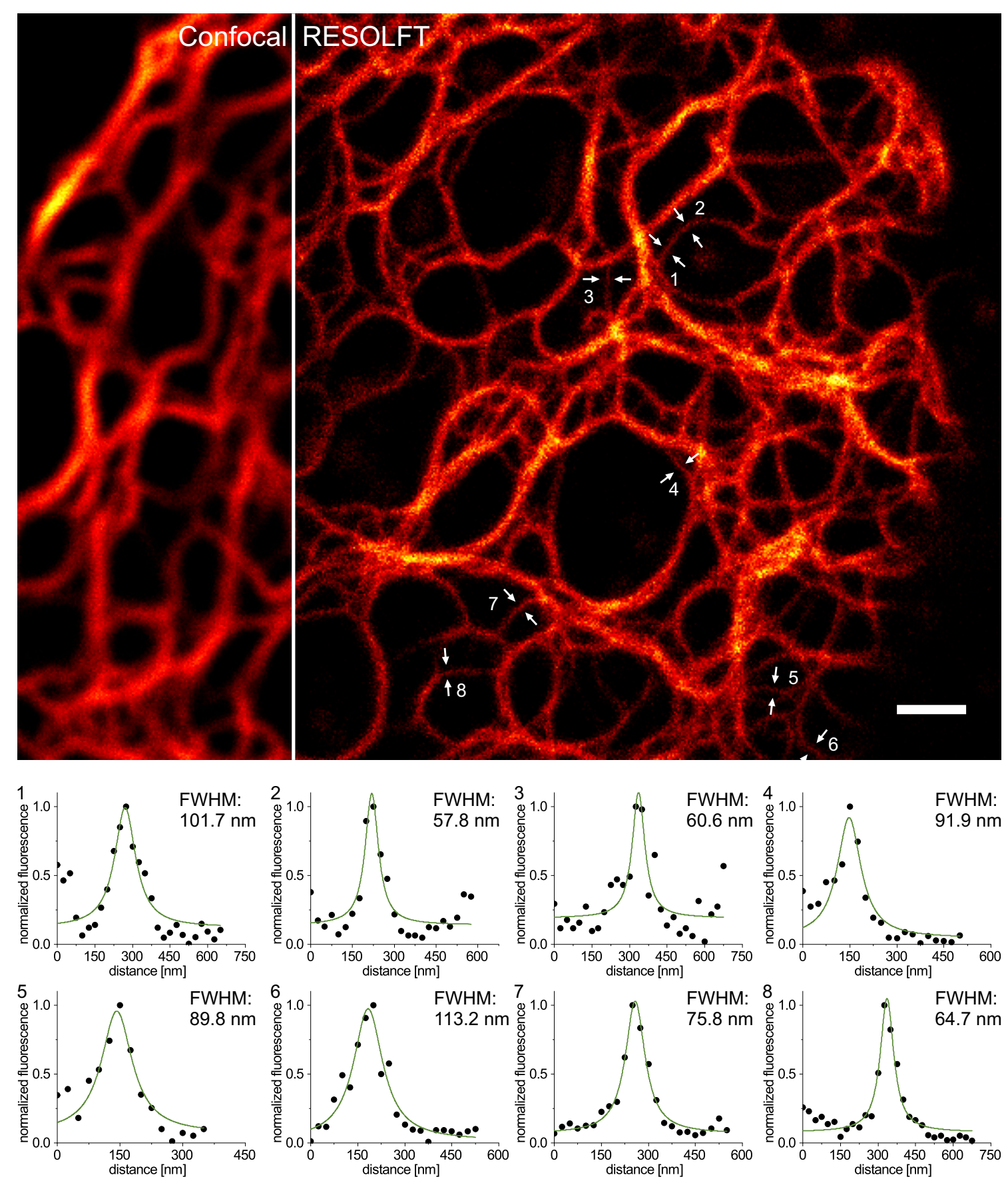

Figure 3.25: RESOLFT microscopy of vimentin-Padron 2.0 fusion constructs with sequential switching sequence. Transiently transfected HeLa cells were imaged with $25 \mathrm{~nm}$ pixel size in pixel step mode. The confocal image was recorded first with $1.0 \mathrm{~kW} / \mathrm{cm}^{2}$ and $120 \mu \mathrm{s}$ pixel dwell time. The RESOLFT image was recorded with sequential switching steps with the following pixel dwell times: $70 \mu \mathrm{s} 23.1 \mathrm{~kW} / \mathrm{cm}^{2}$ activation, $350 \mu \mathrm{s} 1.0 \mathrm{~kW} / \mathrm{cm}^{2}$ doughnut-shaped beam to switch of peripheral fluorophores, and $120 \mu \mathrm{s} 1.0 \mathrm{~kW} / \mathrm{cm}^{2}$ readout. Line profiles were measured at 8 manually selected positions with 3 adjacent lines and modeled with a Lorentzian fit. FWHM of the fits are indicated in the graphs. Scale bar: $1 \mu \mathrm{m}$. 
Therefore, an alternative switching scheme was established in the next step, the corresponding laser sequence is depicted in figure 3.26. This altered approach featured an initial activation step and following doughnut-based inactivation as well, but kept the $405 \mathrm{~nm}$ doughnut active during a prolonged readout step. This was expected to keep peripheral fluorophores in the non-fluorescent off-state as demonstrated in E. coli in section 3.4.1.5 (fig. 3.14). In those measurements a $405 \mathrm{~nm}$ to $488 \mathrm{~nm}$ intensity ratio of 2 was sufficient to switch Padron 2.0 to a residual fluorescence below $5 \%$.

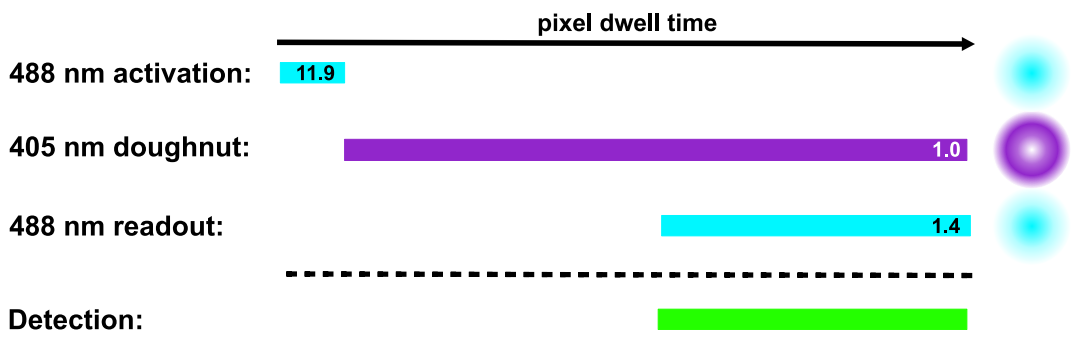

Figure 3.26: RESOLFT microscopy scheme with sequential switching sequence and activation suppressed readout. A RESOLFT image recorded with the illustrated laser sequence is shown in figure 3.27. Numbers are laser intensities in $\mathrm{kW} / \mathrm{cm}^{2}$.

Figure 3.27 shows the resulting RESOLFT image from this approach. With the smallest FWHM featuring $\sim 69 \mathrm{~nm}$ and overall slightly increased values, this altered approach at doubled readout times resulted in similar RESOLFT images, an additional image recorded with this approach is shown in figure 7.11 in the appendix. However, due to the inhomogeneity of vimentin samples, subtle overall resolution differences were difficult to quantify. The image shown was recorded with an equally large field of view as the image in figure 3.25 and the same total pixel dwell time.

As ensemble suppression of peripheral fluorophore activation in principle worked, further imaging experiments elaborated on this approach. Both imaging schemes above featured an initial activation step that switched the majority of the RSFPs to the on-state. This approach was unfavorable because of two reasons. First, this meant that even peripheral fluorophores underwent full switching cycles for two or more adjacent coordinates although these fluorophores optimally were non-fluorescent for these readout times. Second, the laser intensity of the activation step was rather high compared to the following deactivation and readout to keep dwell times at a reasonable level. Unfortunately, this meant that fluorophores that transitioned to the on-state at the beginning of the activation step were excited and therefore at least to some extent susceptible to photobleaching. 

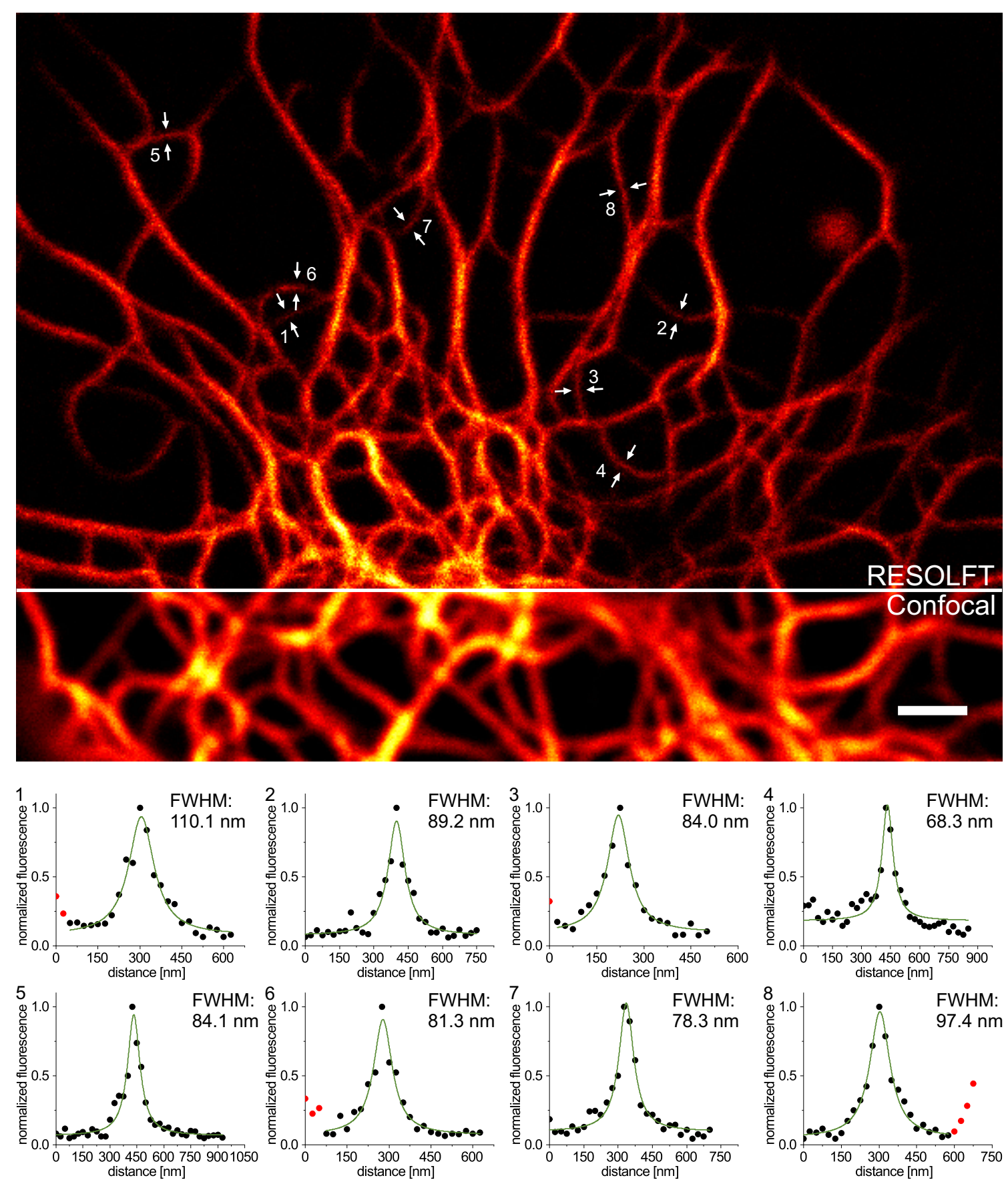

Figure 3.27: RESOLFT microscopy of vimentin-Padron 2.0 fusion constructs with sequential switching sequence and activation suppressed readout. Transiently transfected HeLa cells were imaged with $25 \mathrm{~nm}$ pixel size in pixel step mode. The confocal image was recorded first with $1.4 \mathrm{~kW} / \mathrm{cm}^{2}$ and $240 \mu \mathrm{s}$ pixel dwell time. The RESOLFT image was recorded with sequential switching steps with the following pixel dwell times: $50 \mathrm{\mu s}$ $11.9 \mathrm{~kW} / \mathrm{cm}^{2}$ activation, $250 \mathrm{\mu s} 1.0 \mathrm{~kW} / \mathrm{cm}^{2}$ doughnut to switch off peripheral fluorophores, and $240 \mu \mathrm{s} 1.4 \mathrm{~kW} / \mathrm{cm}^{2}$ readout with simultaneous suppression of fluorescence activation of peripheral fluorophores with the $405 \mathrm{~nm}$ doughnut at $1.0 \mathrm{~kW} / \mathrm{cm}^{2}$. Line profiles were measured at 8 manually selected positions with 3 adjacent lines and modeled with a Lorentzian fit, red data points were excluded. FWHM of the fits are indicated in the graphs. Scale bar: $1 \mu \mathrm{m}$. 

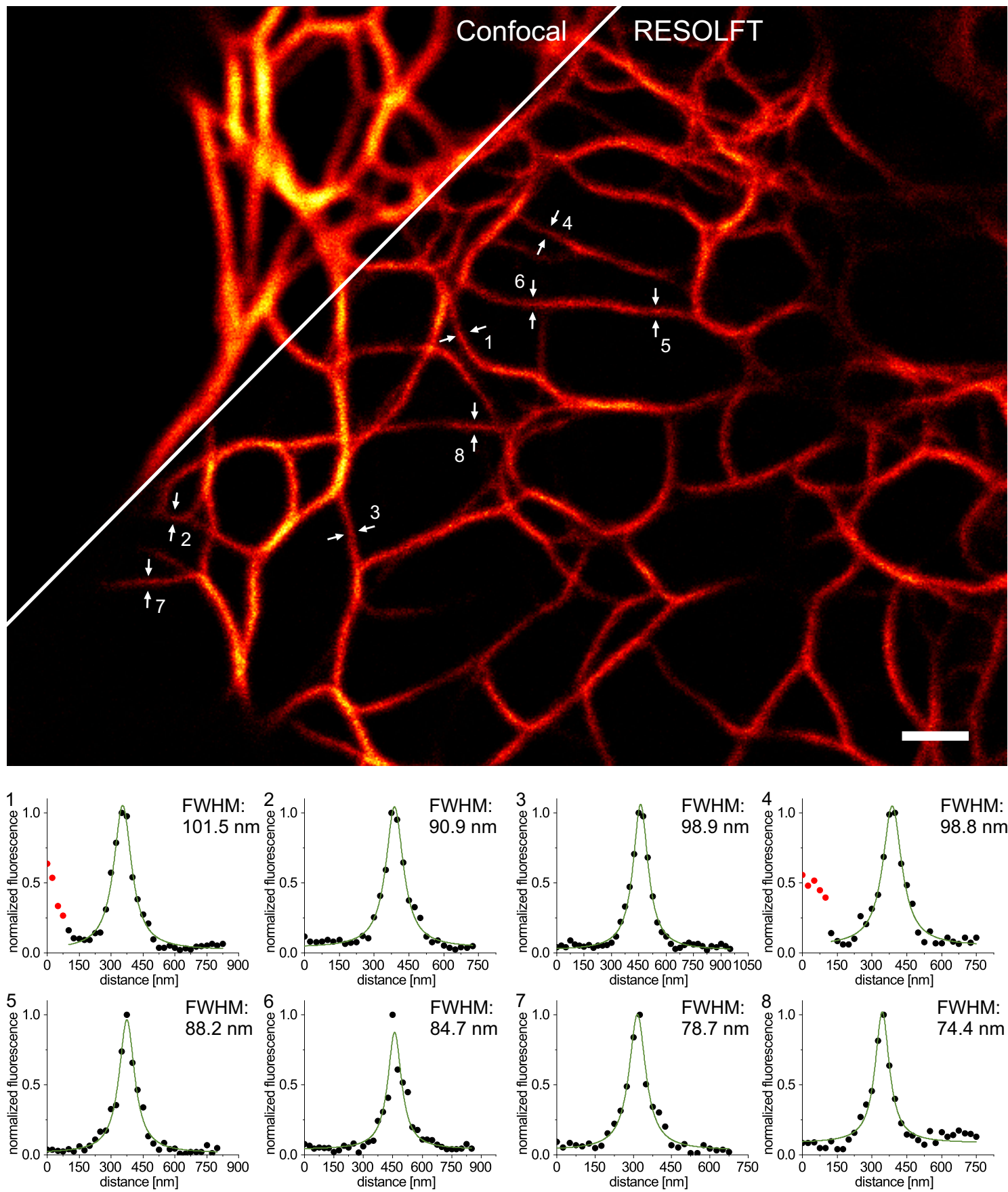

Figure 3.28: One-step RESOLFT microscopy of vimentin-Padron 2.0 fusion constructs. Transiently transfected HeLa cells were imaged with $25 \mathrm{~nm}$ pixel size in pixel step mode. The confocal image was recorded first with $1.4 \mathrm{~kW} / \mathrm{cm}^{2}$ and $120 \mu$ sixel dwell time. The RESOLFT image was recorded by applying both the $405 \mathrm{~nm}$ doughnut at $1.0 \mathrm{~kW} / \mathrm{cm}^{2}$ and the readout laser at $1.4 \mathrm{~kW} / \mathrm{cm}^{2}$ to reach a steady state of central fluorescent RSFPs and non-fluorescent ones in the periphery of the focal spot. Pixel dwell time for this approach was $300 \mu \mathrm{s}$. Line profiles were measured at 8 manually selected positions with 3 adjacent lines and modeled with a Lorentzian fit, red data points were excluded. FWHM of the fits are indicated in the graphs. Scale bar: $1 \mu \mathrm{m}$. 


\section{Results}

To counteract this issue, the activation step was left out entirely in a third, simplified RESOLFT approach (fig. 3.29), where both the doughnut-shaped $405 \mathrm{~nm}$ laser and the readout laser were activated for the complete pixel dwell time. This resulted in a steady-state formation between central on- and peripheral off-state fluorophores. In doing so, peripheral fluorophores were kept in the off-state and were only switched to the on-state by the readout laser once they were located at the central region of the PSF during point-scanning. For this approach to work, the steady-state at any given position has to be reached fast in relation to the scanner movement, because scanning occurs in a continuous movement of the focal spots rather than with discrete steps between coordinates. Conclusively, by this the resolution improvement should mainly depend on the intensity ratios of $405 \mathrm{~nm}$ doughnut and the $488 \mathrm{~nm}$ readout laser, with the only time dependent factor being the time needed to reach the steady-state equilibrium. Thus, acquisition pixel dwell times could in theory be prolonged infinitely and would only be limited by photobleaching and switching fatigue of the RSFP. This approach is termed one-step RESOLFT here.

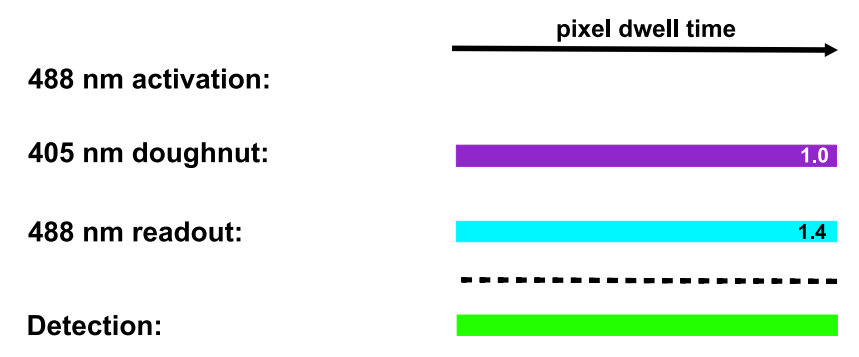

Figure 3.29: RESOLFT microscopy scheme with one-step RESOLFT. A RESOLFT image recorded with the illustrated laser sequence is shown in figure 3.28. Numbers are laser intensities in $\mathrm{kW} / \mathrm{cm}^{2}$.

The RESOLFT image that originated from this approach is shown in figure 3.28. Although the lowest FWHM of the filaments analyzed was $\sim 74 \mathrm{~nm}$ and thus slightly higher than what was shown above, overall resolution improvement was comparable to the classical RESOLFT approach with sequential activation, deactivation, and readout. In addition, pixel dwell time and thus overall imaging time was reduced by almost $50 \%$ from $540 \mu \mathrm{s}$ to $300 \mu \mathrm{s}$. Laser intensity applied to the cell was also the lowest for this approach. The RESOLFT image in figure 3.25 was recorded with $2.1 \mathrm{~J} / \mathrm{cm}^{2}$ at each pixel, while the image from figure 3.27 was recorded with $1.2 \mathrm{~J} / \mathrm{cm}^{2}$. This value was only $0.7 \mathrm{~J} / \mathrm{cm}^{2}$ for the image shown in figure 3.28 . To further establish the simplified switching scheme, transiently transfected HeLa cells with Padron 2.0 labeled nuclear pore complexes were imaged. These structures were rather dim compared to vimentin and resulted in low contrast images with the classical sequential approach. Figure 3.30, however, shows good resolution improvement with RESOLFT which facilitated separation of individual nuclear pore complexes. For this purpose, readout time was increased to $500 \mu \mathrm{s}$.

After all, it could be shown that Padron 2.0 is suitable for RESOLFT nanoscopy. In addition, due to the dominant off-switching characteristics under simultaneous irradiation with both 405 and $488 \mathrm{~nm}$, it was possible to establish a new mode of RESOLFT nanoscopy termed 
one-step RESOLFT. This approach is expected to simplify the establishment for new samples because the defining parameter is the power ratio of doughnut and readout laser intensity, and the steps for activation and deactivation were left out entirely and replaced by the formation of a steady-state of the on- and off-state of RSFPs. Moreover, the low switching fatigue of Padron 2.0 allowed for long readout times in this approach, which aids imaging of dim structures.

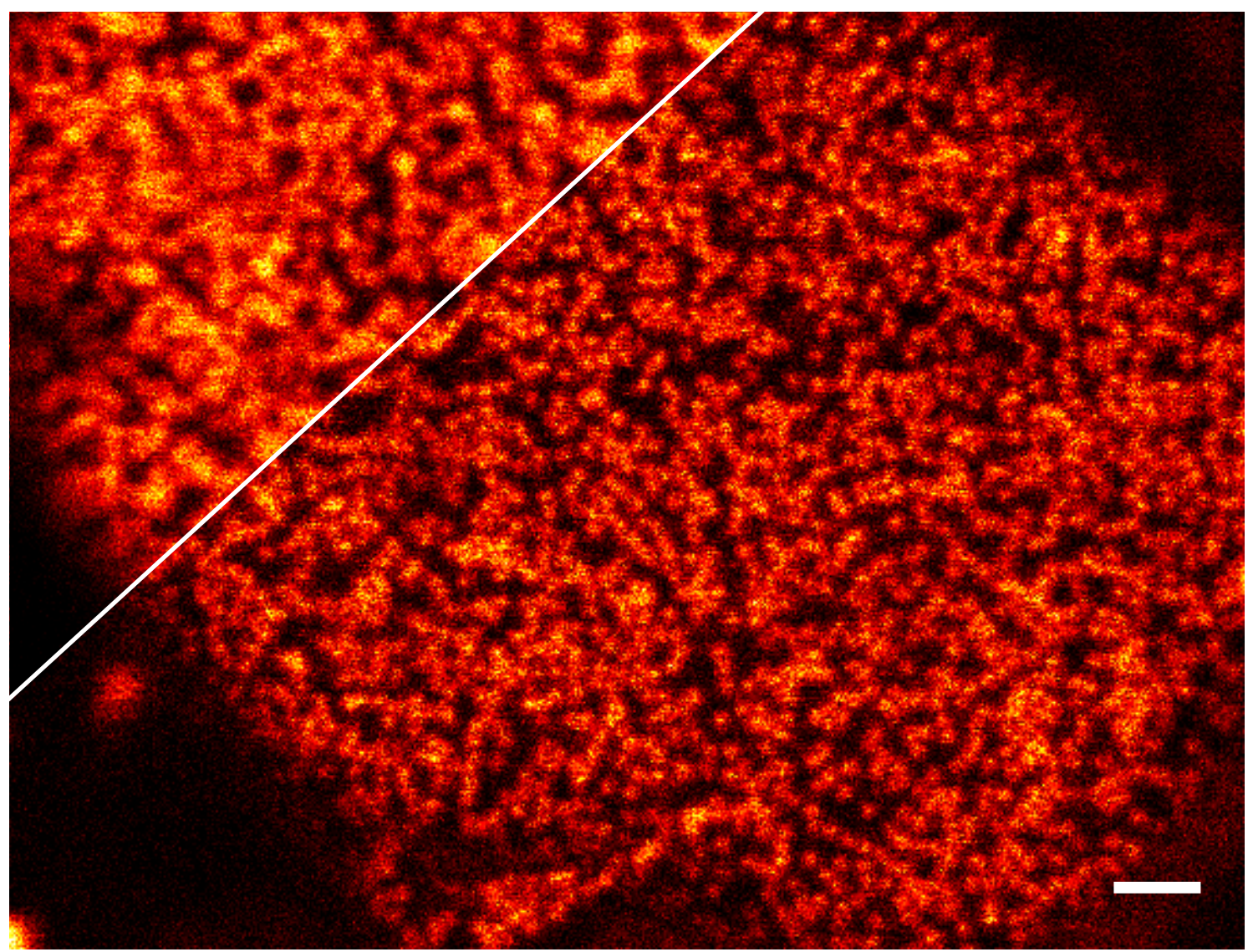

Figure 3.30: One-step RESOLFT microscopy of Padron 2.0-Nup50 fusion constructs. Transiently transfected HeLa cells were imaged with $25 \mathrm{~nm}$ pixel size in pixel step mode. The confocal image was recorded first with $1.7 \mathrm{~kW} / \mathrm{cm}^{2}$ and $400 \mu \mathrm{s}$ pixel dwell time. The RESOLFT image was recorded by applying both the $405 \mathrm{~nm}$ doughnut at $0.9 \mathrm{~kW} / \mathrm{cm}^{2}$ and the readout laser at $1.7 \mathrm{~kW} / \mathrm{cm}^{2}$ to reach a steady state of central fluorescent RSFPs and non-fluorescent ones in the periphery of the focal spot. Pixel dwell time of the RESOLFT image was $500 \mu \mathrm{s}$. Scale bar: $1 \mu \mathrm{m}$.

\subsubsection{Comparison of sequential and one-step RESOLFT imaging}

Suppressing fluorescence activation during readout or utilizing steady-state formation during simultaneous $405 \mathrm{~nm}$ doughnut and Gaussian-shaped $488 \mathrm{~nm}$ irradiation was shown to be feasible. However, it was still unclear how the latter approach performed with regards to bleaching during time-lapse imaging. Omitting the initial activation step was expected to lower photobleaching of activated peripheral chromophores as these were forced to reside 


\section{Results}

predominantly in the off-state. The contribution of switching fatigue on the other hand remained elusive. So far, simultaneous irradiation was assumed to suppress full switching cycle progression of fluorophores within the PSF range at every coordinate, but could potentially also induce constant cycling of fluorophores between their on- and off-state. If the rate for activation was too high in the latter approach, RSFPs could even undergo an increased number of switching cycles although the ensemble of fluorophores would appear as mostly non-fluorescent. To test if this cycling was detrimental to switching fatigue under the conditions chosen, 30 frames were recorded for both sequential and one-step RESOLFT mode (fig. 3.31). The former was recorded with $70 \mu \mathrm{s} 11.9 \mathrm{~kW} / \mathrm{cm}^{2}$ activation, $350 \mu \mathrm{s}$ $1.2 \mathrm{~kW} / \mathrm{cm}^{2}$ doughnut-patterned deactivation to switch off peripheral fluorophores, and $120 \mu \mathrm{s} 1.0 \mathrm{~kW} / \mathrm{cm}^{2}$ readout, while the latter was imaged with $300 \mu \mathrm{s}$ pixel dwell time with the $405 \mathrm{~nm}$ doughnut at $1.0 \mathrm{~kW} / \mathrm{cm}^{2}$ and the $488 \mathrm{~nm}$ readout laser at $1.2 \mathrm{~kW} / \mathrm{cm}^{2}$. Bleaching was analyzed in a central region as depicted in figure 3.31a, b to exclude fluorophores from the analysis that entered the field of view as a result of drift or vimentin dynamics.

Both approaches could achieve FWHM values of single filaments of approximately $90 \mathrm{~nm}$ (fig. 3.31d, e), but bleaching over the course of 30 frames differed. While the last recorded frame of the one-step approach could still provide about $60 \%$ of the counts of the first frame, time-lapse images recorded with sequential switching mode were bleached to below $30 \%$ (fig. 3.31c). Unfortunately, resolution improvement was impaired for both approaches after 30 frames.

The simplified one-step switching approach for RESOLFT microscopy could indeed improve both dwell times and bleaching for time-lapse imaging. Imaging time was reduced by the same factor described in section 3.5.2. For the $8 \times 8 \mu \mathrm{m}$ region shown in figure 3.31 this meant a reduction from approximately 28 minutes to about 15 minutes for the 30 frames recorded.

In conclusion, Padron 2.0 was improved in comparison to the original Padron in almost every aspect. The analysis of switching characteristics has shown that Padron 2.0 reached a lower switching background than Padron with a lower dose of $405 \mathrm{~nm}$ light and could sustain significantly more switching cycles. While spectral analysis displayed a hypsochromic shift of absorption, excitation, and emission spectra, the extinction coefficient, quantum yield, and, consequently, the molecular brightness were lower in Padron 2.0. This impairment was made up for by its enhanced expression and maturation at $37^{\circ} \mathrm{C}$, which was a major drawback of the original Padron. RESOLFT nanoscopy of vimentin structures with Padron 2.0 in living HeLa cells achieved a resolution below $60 \mathrm{~nm}$, and the improved switching characteristics allowed for the introduction of one-step RESOLFT microscopy, which constitutes a simplified irradiation sequence at shorter pixel dwell times with less bleaching. The following chapter will discuss the results presented here in a wider scientific context. 

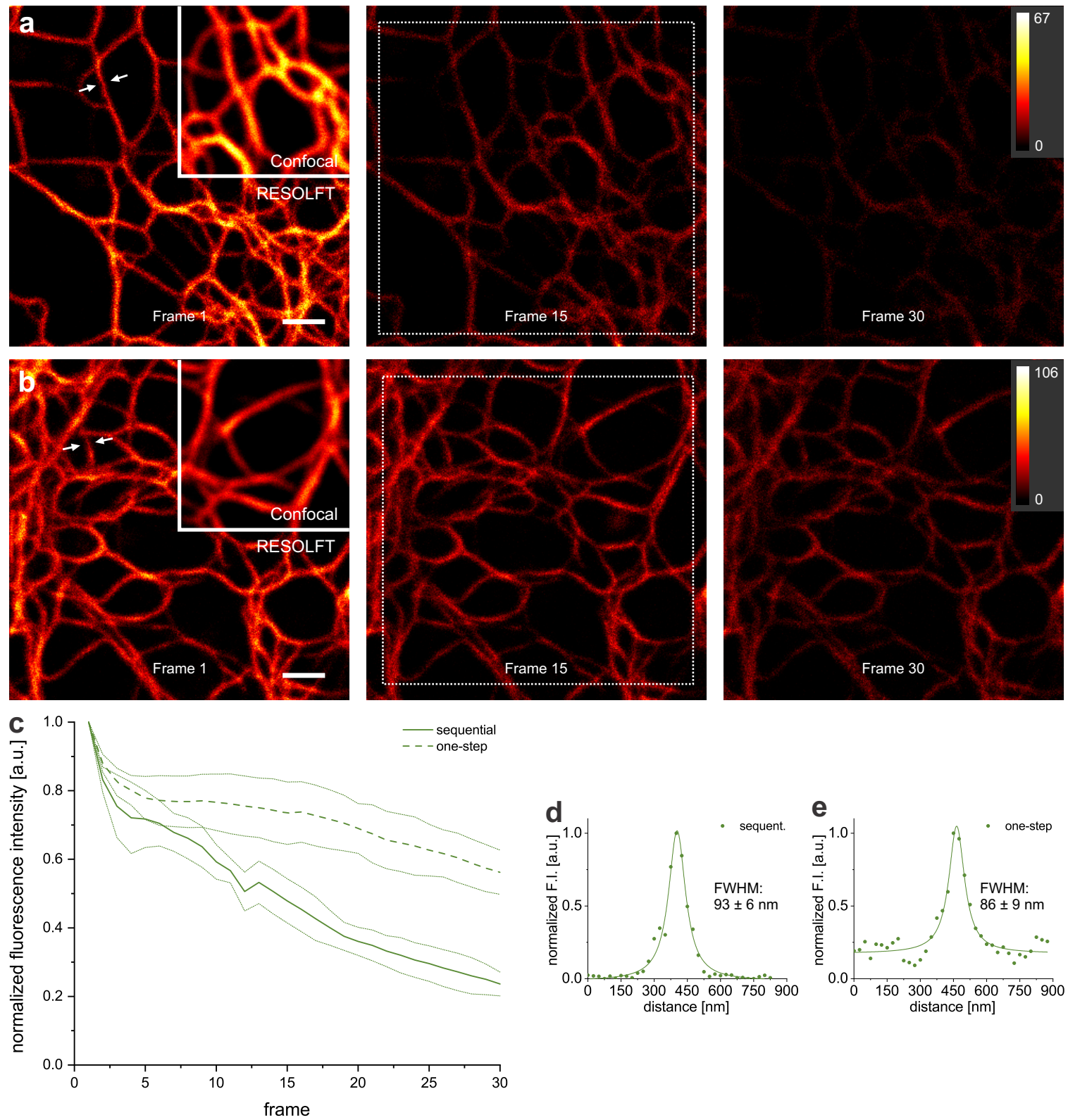

Figure 3.31: Bleaching of sequential versus one-step RESOLFT microscopy. 30 consecutive frames were recorded with the (a) classical sequential or (b) one-step switching approach with simultaneous irradiation for RESOLFT microscopy. Frame 1 has an inset of the confocal image recorded before the time-lapse. All images are raw data displayed with consistent lookup tables within sequences that are shown in frame 30. (c) Total counts measured in a central region of interest similar to the boxed regions of frame 15 of a and $b$ were normalized and averaged for 3 different time-lapses for both approaches. Dotted lines illustrate standard deviations. (d, e) 3 adjacent line profiles were measured in the first frame and fitted with a Lorentzian fit at the positions marked by arrows in a or b, respectively. Scale bars: $1 \mu \mathrm{m}$. 


\section{Discussion}

\subsection{Improvement of the automated screening microscope}

In the course of this thesis, the automated screening microscope used for the assessment of mutated fluorescent proteins as well as detailed characterization was improved in two central aspects: Both colony sampling and the autofocus were time consuming steps which limited the throughput during screening of large mutant libraries. With fast switching schemes taking place in the range of microseconds to milliseconds most of the screening time was owed to microscope operation. By reducing false positive and false negative measurements, both speed and precision were improved with the new sampling mode. Although the user input required before a screen took more time because plates had to be recorded with a separate instrument, this loss of time was easily made up for by the increased number of colonies that could be sampled on each plate. This reduced the number of plates and thus user interactions for an equal number of mutants analyzed. By measuring every colony in its center, the new sampling also increased the quality of data, and excluding overlapping colonies beforehand resulted in a lower workload after screenings.

The improvement of the autofocus, however, came with some caveats. As the faster autofocus required a calibration to relate its time dimension to the position along the optical axis of the microscope, this introduced a new source of error. This calibration had to be repeated for every measurement as the linear regression of time and position was different for varying focus parameters such as range in z covered or position of the colony relative to this range. Especially the latter factor was a source of error, which albeit could be easily avoided. If the position of maximal intensity was situated too close to the start or end of the focal range, the linear regression was not sufficient to adequately calculate the focal position as acceleration or deceleration of the stage happened while the fluorescence was peaking. This could be circumvented by using a focal range large enough, and future versions of the autofocus could eradicate this shortcoming by employing a more sophisticated calibration.

Another factor was the increased light dose applied with the new autofocus, in which the irradiation occurred over the full focal range, while the old autofocus only probed fluorescence at certain positions. Fast switching proteins would add another factor influencing the position of the fluorescence peak, rendering the calculation of the focal position non-trivial. This was, however, not the case for the measurements performed so far as the PMT was sensitive enough so that the power of the laser could be lowered to non-invasive levels.

In short, both colony sampling and autofocusing were critically improved, which allowed for higher throughput in screenings after mutagenesis. Future iterations of the software could increase both robustness and ease of use to further increase the benefit of these functions to the workflow. 


\subsection{Generation of a new Padron variant}

The results presented in this thesis have shown that Padron 2.0 is an improvement over the original Padron and Kohinoor, its robust switching characteristics render it particularly well-suited for RESOLFT nanoscopy applications. It is also the best-performing positively switching RSFP to date. This chapter will discuss the relevant improvements in detail and in a wider scientific context. All numbering of amino acid positions refers to Dronpa if not stated otherwise.

Beforehand, it is necessary to evaluate the data generated in comparative measurements for Padron and Kohinoor in relation to the original publications. The results obtained for switching characteristics differ in certain aspects and require clarification.

\subsubsection{Padron}

After the publication of asFP595 [118], Padron [120] and rsCherry[122] were the first engineered positively switching RSFPs. However, practical applications of Padron have been scarce [162-165]. With regards to Padron, this is in part due to the poor performance of switching characteristics. In the presented comparative analysis of Padron 2.0 and its progenitors, the shortcomings of Padron for nanoscopy become apparent. In the following this will be discussed with regards to the published characteristics of this protein.

Spectral properties such as absorption maxima of the on- and off-state spectra or the emission maximum and the extinction coefficient of Padron were equivalent to the published data [120], minor differences can be attributed to variations in experimental procedures. For example, switching prior to spectral analysis was performed in a cuvette at the final concentration at which measurements were performed in this thesis, while Andresen et al. switched Padron in a small volume of $2 \mu \mathrm{l}$ and diluted the sample before spectral analysis. However, differences of peak positions are still negligible and within the range of the sampling rate, while on- and off-state absorption spectra are nearly identical.

Interestingly, certain switching characteristics differed depending on the parameters analyzed, although overall results are comparable: Equilibrated ground-state fluorescence was determined to be at $5 \%$ of the on-state fluorescence intensity by Andresen et al., while the same parameter was found to be approximately $10 \%$ in this thesis. Relaxation half-time from the on-state was equally different with 150 minutes published and 300 minutes here. For these parameters, the model system was most probably the reason for different results. Andresen et al. measured both characteristics in E. coli where exact concentrations remain elusive, while the data in this thesis were generated with purified protein in solution. Repeated empirical observations made during the course of this thesis had shown that the spectral properties of the equilibrated state of Padron variants were dependent on the concentration of proteins (data not shown), and interaction of Padron variants varied similarly with slowly adapting kinetics (sec. 3.4.4.2). Another factor that influences the relaxation of the switching state is the temperature [144]. However, Andresen et al. determined the relaxation half-time 


\section{Discussion}

at $22^{\circ} \mathrm{C}$, which was only $1{ }^{\circ} \mathrm{C}$ higher than the measurement conditions in this thesis.

In contrast, switching parameters were determined in E. coli in both works. Still, the results differed: Andresen et al. determined the ensemble residual off-state fluorescence intensity of Padron to be as low as $0.7 \%$ as opposed to $2.3 \%$ in this thesis. Switching fatigue was also less pronounced in the former publication, although there the off-switching step was recorded with both wavelengths, which was shown to be unfavorable for Padron (sec. 3.4.1.5). These differences are founded on the profoundly different power regimes applied to the samples. Andresen etal. recorded switching curves with a widefield microscope with light intensities in the range of $500 \mathrm{~mW} / \mathrm{cm}^{2}$. Data presented here were recorded in the lower range of $\mathrm{kW} / \mathrm{cm}^{2}$, which is more representative of light intensities applied in confocal microscopy or RESOLFT nanoscopy with pixel dwell times below $1 \mathrm{~ms}$ [51, 124]. While the activation half-time in the original publication was in the range of seconds, this parameter resided in the millisecond range at the intensities used here. This discrepancy in data acquisition resulted in different interpretations of switching capabilities of Padron. Supplementary data of the former publication lists equilibrium fluorescence intensities of Padron relative to the full on-state at simultaneous irradiation with 405 and $488 \mathrm{~nm}$. Although only intensity ratios and no absolute intensities were reported, it can be assumed that irradiation was in the range of $\mathrm{mW}$ to $\mathrm{W} / \mathrm{cm}^{2}$ because the same samples were used for the generation of switching curves. Under these conditions, Andresen et al. found that Padron fluorescence was at $2.5 \%$ of the maximal fluorescence after irradiation at a $488: 405 \mathrm{~nm}$ intensity ratio of 0.5 . For a nearly identical intensity ratio in the low $\mathrm{kW} / \mathrm{cm}^{2}$ range, figure $3.14 \mathrm{a}$ shows a residual fluorescence of about $17 \%$ and, more importantly, opposing results at higher $405 \mathrm{~nm}$ intensities. These results suggest that Padron behaves differently at low and high intensities. While ensemble activation of fluorophores could be suppressed in the range of $\mathrm{W} / \mathrm{cm}^{2}$, light of $405 \mathrm{~nm}$ could not compensate the activation facilitated by light of $488 \mathrm{~nm}$ in the range of $\mathrm{kW} / \mathrm{cm}^{2}$. In this context, it is of interest that Duan etal. could show two distinct bleaching mechanisms for IrisFP whose occurrence depended on the light intensities applied [176]. Lower intensities resulted in oxygen dependent bleaching while intensities above $0.1 \mathrm{~kW} / \mathrm{cm}^{2}$ resulted in oxygen independent bleaching. This threshold is situated between the intensities applied in this thesis and by Andresen et al., suggesting that the results described above differed because of the influence of two different bleaching mechanisms.

After all, Padron displayed compelling switching characteristics at low intensities in the range of $\mathrm{mW} / \mathrm{cm}^{2}$ [120], but the results presented in this thesis show that these properties cannot be transferred to higher intensities in the range of $\mathrm{kW} / \mathrm{cm}^{2}$. This discrepancy is particularly important for the applicability of Padron under RESOLFT microscopy conditions and highlights the necessity to improve the switching characteristics of Padron for nanoscopy applications. Kohinoor as a variant of Padron was a step in this direction. 


\subsubsection{Kohinoor}

Kohinoor was published in 2015 by Tiwari et al. as a brighter, faster, and more stable variant of Padron [121], and its switching performance was compared to other variants of Padron in this thesis. In part, the results of this comparison displayed characteristics different from the original publication of Kohinoor, with some of them posing a considerable limitation for its application in point-scanning RESOLFT nanoscopy. These differences between the published results and the findings in this study will be explained and classified below.

The majority of spectral characteristics was consistent between both data sets. Peak positions for on- and off-state absorption spectra as well as the on-state emission differed only marginally. The value of $\mathrm{p} K_{a 2}$ was identical, while $\mathrm{p} K_{a 1}$ differed by 0.6 , possibly based on different protocols. Tiwari et al. recorded $\mathrm{pH}$-dependent fluorescence in the on-state while data presented here were recorded in the equilibrated state.

In contrast, the reported extinction coefficient and molecular brightness varied distinctively. The former was reported by Tiwari et al. to be $62,900 \mathrm{M}^{-1} \mathrm{~cm}^{-1}$ and thus nearly 1.5 -fold higher than the published value of Padron. However, these two values were measured at entirely different $\mathrm{pH}$-values: Andresen et al. calculated the extinction coefficient at $\mathrm{pH} 7.5$, while Tiwari et al. recorded the absorption at $\mathrm{pH} 10$ and compared these two results. The $\mathrm{pH}$ value, however, has a tremendous influence on the outcome: Figure 3.19 shows how the absorption spectrum of the equilibrium ground-state changed with increasing $\mathrm{pH}$, shifting the ratio of protonated and deprotonated absorption peaks towards the latter one at high values. This $\mathrm{pH}$ response was observed for all Padron variants presented here, and Tiwari et al. showed the same dependency for the on-state absorption spectra of Kohinoor. Hence, the comparison made in the Kohinoor publication overestimated differences between Padron and Kohinoor concerning their brightness, which was reported to be approximately 1.6-fold higher for the latter one. In contrast, the extinction coefficient calculated for Kohinoor in this thesis was 5 times lower at $\mathrm{pH} 7.5$ than the published value at $\mathrm{pH} 10$. The resulting molecular brightness was still 3 times lower compared to Padron.

A comparison of switching parameters between the published data and results presented in this thesis is necessarily to remain coarse. While switching kinetics for the data presented here were measured in bacterial colonies with focused lasers in a non-scanning confocal setup, Tiwari et al. recorded switching data with a confocal laser scanning microscope. Samples were either purified protein in a polyacrylamide layer or RSFP expressing E. coli immobilized in low-melting agarose. Unfortunately, Tiwari et al. reported insufficient details to reproduce the measurements. For photobleaching, they stated that samples were recorded in a $64 \times 64$ pixel array with $2 \mu \mathrm{s}$ pixel dwell time at a $488 \mathrm{~nm}$ intensity of $7 \mathrm{~kW} / \mathrm{cm}^{2}$. However, the pixel size was not reported and thus the actual field of view imaged remains elusive. Still, the half-time ratios of Padron and Kohinoor for bleaching to $50 \%$ were in good accordance with the data listed in table 3.4.

In contrast, the data reported for switching fatigue was rather different. Table 3.4 lists the number of switching cycles Padron and Kohinoor could perform upon cycling between 5 or 


\section{Discussion}

$10 \%$ residual fluorescence and $95 \%$ of the full on-state. Under these conditions, Kohinoor could perform approximately twice as many cycles as Padron at both $488 \mathrm{~nm}$ intensities applied before the on-state fluorescence was bleached to $50 \%$. Tiwari etal. reported an improved switching fatigue performance where Kohinoor could perform 25 times more cycles than Padron. The data this result was based upon was recorded at comparable intensities in the low $\mathrm{kW} / \mathrm{cm}^{2}$ range in an array of $256 \times 256$ pixels, but no detailed irradiation times were listed except for the statement that minimal times for cycling were applied for every protein. These two approaches differed fundamentally. While laser positions were fixed for the data presented in table 3.4 to ensure constant and equal irradiation of RSFPs, Tiwari et al. measured data with a laser scanning confocal microscope. This inevitably resulted in alternating intensities and irradiation breaks for single fluorophores which is likely to affect switching fatigue [177].

Switching kinetics results were also found to be conflicting between datasets from Tiwari et al. and this thesis. While Kohinoor proved to be the faster protein with regards to $488 \mathrm{~nm}$ induced activation in both the publication and based on the data presented here, the sensitivity to $405 \mathrm{~nm}$ light was found to be different. Figure 3.9 shows that Padron reached lower residual fluorescence faster than Kohinoor with a $405 \mathrm{~nm}$ intensity of $4.1 \mathrm{~kW} / \mathrm{cm}^{2}$ in bacterial colonies. Based on the time constant for off-switching of purified proteins in polyacrylamide layers reported by Tiwari et al., Kohinoor switched off more than 3 times faster than Padron at a $405 \mathrm{~nm}$ intensity of $0.8 \mathrm{~kW} / \mathrm{cm}^{2}$, albeit off-switching curves were recorded with simultaneous irradiation of 405 and $488 \mathrm{~nm}$. However, no statements about pixel size or dwell times were made for these data in the methods section, a circumstance which precludes a detailed comparison. Assuming that switching curves were recorded by Tiwari et al. in a nonscanning approach similar to the automated screening microscope used in this thesis is highly unlikely. On-switching curves for purified proteins in a polyacrylamide layer were recorded at $25 \mathrm{~W} / \mathrm{cm}^{2}$ and resulted in a time constant of 7.9 seconds. However, for RESOLFT images Kohinoor was activated at a similar intensity of $36 \mathrm{~W} / \mathrm{cm}^{2}$ for $80 \mu \mathrm{s}$.

All in all, both data sets discussed above support the interpretation that Kohinoor has been improved in comparison to Padron, albeit to a different extent. The use of different model systems and experimental approaches makes a direct comparison difficult. However, different performances of the off-switching capabilities of Kohinoor pose the question how well onand off-states of Kohinoor can actually be separated in RESOLFT imaging, supporting the development of the new Padron variants presented here. The data presented in this study suggests that the switching performance of Kohinoor has been overestimated with regards to its performance in RESOLFT nanoscopy.

\subsubsection{Generation of the new Padron variants}

Padron 2.0 differs from its template Padron in 11 positions, which had pronounced effects on its characteristics. As no crystallographic structure of Padron 2.0 was generated for this thesis, the structural changes remain elusive. However, some of the positions mutated are 
conserved in related proteins and have been the focus of extensive research. In the following, the mutations introduced with the new Padron variants will be compared to similar mutations that were described for other proteins in the literature. The comparison will focus on finding potential explanations for the effects observed in the new Padron variants. Because intermediate Padron variants were not characterized in detail, not every effect can be assigned to a single mutation. In addition, there are only few publications about detailed mechanistics of positively switching RSFPs, and all of them focused on analysis of Padron or Kohinoor without performing further mutagenesis [61, 140]. Contrary to that, Dronpa [119] and EosFP [106] as well as variants based on either of these proteins were substantial to publications that focused on elucidating the switching mechanism [178, 179] or fluorescent protein characteristics [180]. While Dronpa was indeed the template from which Padron was generated, the latter exhibited an inverted light response [120]. Any observations made for the negatively switching Dronpa could be absent in Padron as both proteins exhibit a different structural basis for the switching mechanism [140]. EosFP on the other hand is a non-switchable photoconvertible fluorescent protein [106] that has $73 \%$ sequence identity with Padron. Among the identical amino acids are key residues which interact with the chromophore. However, due to inherently different photophysics of photoconvertible and -switchable proteins, the functions of those amino acids are not necessarily shared or of equal importance.

a

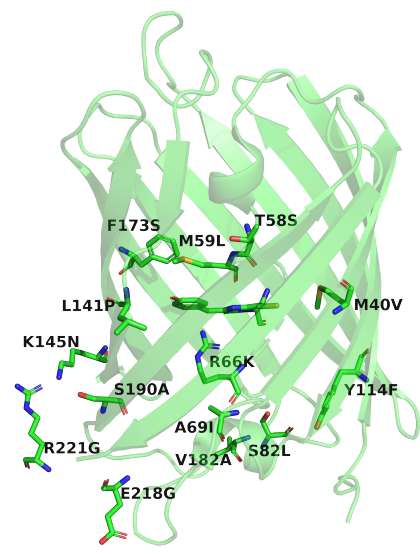

b

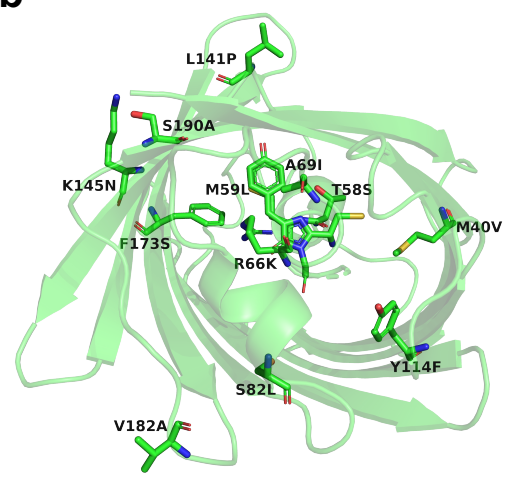

Figure 4.1: Mutated positions of new Padron variants in crystallographic structure of Padron. (a) Amino acids in stick representation were mutated as indicated by the respective labels. The structure shown is the on-state of Padron (PDB ID: 3ZUJ, [61]). Glu218 and Arg221 were not part of the solved structure and were added by hand. (b) Bottom view of the structure shown in a. Met59 is located behind the chromophore in this view.

Figure 4.1 gives an overview of the positions of mutations in the Padron structure. The side chains of Tyr114, Leu141, and Ser190 are outside of the $\beta$-barrel, whereas Ser82 points to the inner part of the structure but is part of a short $\alpha$-helix at the bottom of the barrel, distant to the chromophore. Met40, Thr58, Arg66, Ala69, and Phe173 are positioned in close proximity 
of the chromophore and are potentially involved in chromophore positioning, or at least close to amino acids that are. Glu218 and Arg221 were not part of the solved crystal structure of Padron [61]. These two residues close to the C-terminal end of the primary structure are thus likely part of an unstructured loop in this variant [85].

\subsubsection{Influence of arginine 66 , alanine 69 , and leucine 141}

Of the 11 positions mutated in Padron 2.0, Arg66 and Ala69 were the most crucial ones for adapting the protein for RESOLFT microscopy. R66K resulted in slower switching half-times to the on-state, but in turn apparently eliminated the bright intermediate state and greatly improved off-switching under simultaneous irradiation.

Ala69 was initially subjected to site-directed mutagenesis as its mutation to valine had been reported to improve expression at $37^{\circ} \mathrm{C}$ in EosFP [169]. A69C resulted in improved maturation as anticipated, but at the same time caused a progressive change of switching performance over the course of multiple cycles. This was attributed to the introduction of cysteine, whose side chain is susceptible to sulfoxidation, which has been shown to play a role in switching fatigue associated bleaching [176]. Position 69 was thus changed to valine with pa89, resulting in equally improved maturation (fig. 3.5a, c). Interestingly, Kohinoor retained the alanine at this position but still matured faster than Padron, suggesting that other amino acid configurations are also critically involved in this process. Ala69 was ultimately changed to isoleucine in a Padron 2.0. This was necessary because Arg66, which is located closely to position 69, was mutated to lysine and impaired switching fatigue, a circumstance that could be rescued with A69I. This relation of Arg66 and Ala69 has been reported by various publications for other proteins [181, 182]. In EosFP, Arg66 interacts with the carbonyl group in the imidazolinone ring of the chromophore [183], whereas this interaction is absent in Dendra2 (67\% sequence identity with Padron) and Arg66 builds a salt-bridge to Glu212 [181] (corresponding to Glu211 in Padron). Adam et al. proposed the presence of threonine at position 69 to be responsible for this altered orientation [181], and this relationship has been further examined by Berardozzi et al. [180]. They could show that the A69T mutation in mEos2 was sufficient to alter the orientation of Arg66, while T69A in Dendra2 had the opposite effect. Threonine was able to turn the orientation of Arg66 by forming a hydrogen bond with the $\mathrm{N}_{\varepsilon}$ of Arg66. In mEos2, A69T resulted in a hypsochromic shift for absorption and fluorescence emission peaks. This shift was present in bsDronpa as well, although it was not attributed to its A69T mutation in the publication [120]. Berardozzi etal. also reported an increase of the absorption peak of the protonated chromophore and thus a stabilization of this form in mEos2 resulting from A69T [180]. Both the blue-shifted spectra and altered peak ratios of the absorption spectra with a more pronounced peak of the protonated form were observed for the new Padron variants as well (tab. 3.6) although it is unclear if this was caused by the mutations introduced to Arg66 and Ala69. Both positions were unaltered in Kohinoor displaying the same effects, albeit in part to a lesser extent. Interestingly, KikGR1 has a valine at position 69 and Arg66 is in 
contact with the chromophore [184]. As valine has an non-polar side chain like alanine and cannot form a hydrogen bond to Arg66, this effect is expected. Likewise, the replacement with isoleucine in Padron 2.0 only increased the size of the side chain and did not alter polarity.
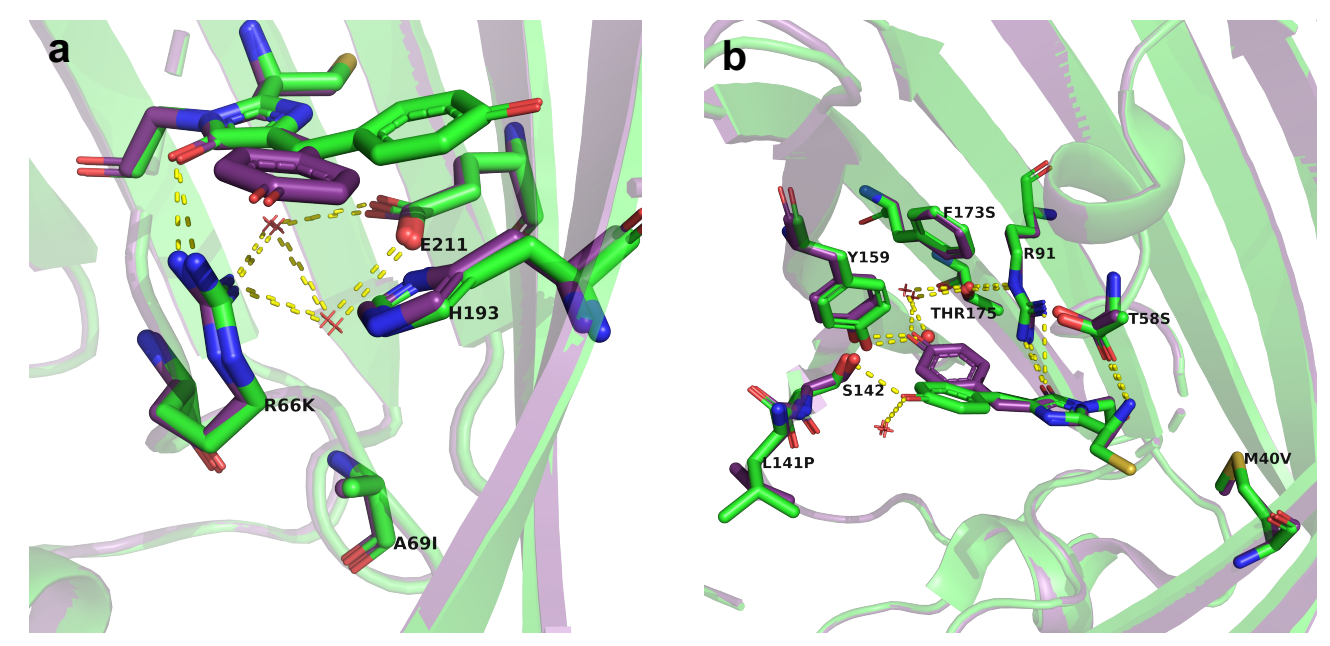

Figure 4.2: Mutated positions in the chromophore vicinity of Padron. (a) Arg66 and Ala69 were situated below the chromophore and mutated according to the label. Arg66 is part of an hydrogen bonding network involving the carbonyl group of the chromophore imidazolinone ring, Glu211 and two water molecules. (b) Positions mutated above the chromophore with important amino acids in direct vicinity. Green and purple coloring represent the on- and off-state of Padron, respectively (PDB IDs: 3ZUJ and 3ZUF, [61]). The red sphere represents a structural water molecule that is absent in the off-state structure.

Li et al. postulated based on molecular dynamics simulations that the chromophore of Dronpa more likely resides in the trans-state if Arg66 interacts with the carbonyl group of the imidazolinone ring [178]. If this statement is true for Padron as well is unclear: While Arg66 and His193 are structurally rearranged in Dronpa during switching events [125, 139], both amino acids maintain nearly identical orientations in Padron (fig. 4.2, [61]). Arg66 was replaced with lysine in Padron 1.9, 2.0, and 2.1, which lacks the $\mathrm{N}_{\varepsilon}$ and thus cannot build the same hydrogen bonds. The terminal amino group of the side chain on the other side is still capable of forming hydrogen bonds. DsRed [94] and mOrange [98], which is based on DsRed, both have a lysine at the corresponding position and a valine at position 69 . The orientation of the lysine, however, is substantially different. In mOrange, Lys70 (corresponding to Arg66 in Padron) interacts with the chromophore carbonyl group [99], while in DsRed Lys70 forms a salt-bridge with Glu215 (corresponding to Glu211 in Padron) [185]. A possible explanation for this altered orientation is the difference at position 197 in both proteins, which corresponds to position 193 in Padron. DsRed has a serine at this position that forms a hydrogen bond with the amino group of Lys70. In mOrange, this serine has been replaced with isoleucine with a non-polar side chain [186]. This suggests a similar relation of Lys70 to position 197 as it was shown for Arg66 and Ala69. Padron and all variants presented here have a histidine at position 193, which is larger than serine but potentially could still form 


\section{Discussion}

hydrogen bonds [187]. Interestingly, the orientation of Lys70 appears to be decisive for the presence of bleaching processes involving decarboxylation of Glu215, a pathway that exists in DsRed [188] but is absent in mOrange [186, 189]. Similar mechanisms have been proposed in relation to Arg66 as well in other proteins [176, 180, 190]. In addition, Arg66 has been proposed to stabilize a transient dark state in IrisFP, mEos2, and Dendra2 by donating a proton to a $\mathrm{sp}^{3}$-hybridized carbon atom of the chromophore methine bridge [180, 190, 191]. If this dark-state was present as well in the Padron variants, it would be unresponsive to light of $405 \mathrm{~nm}$ due to the non-planarity of the chromophore resulting in the interruption of the conjugated $\pi$-system. Mutation of Arg66 would influence the formation of this state which could explain the improved capabilities of new Padron variants to switch off under simultaneous illumination with 405 and $488 \mathrm{~nm}$ (fig. 3.6c).

The dynamics described here were found mostly in non-switchable proteins with chromophores that have a defined position within the barrel. How these findings transfer to switchable fluorescent proteins, however, remains elusive and would require further analysis. For Dronpa, it could be shown that weakening of the interaction of Arg66 and the chromophore precedes cis-trans isomerization [179]. As described above, this is accompanied by structural rearrangement of Arg66, whereas chromophore isomerization in Padron takes place with only minimal structural changes $[61,140]$. Brakemann et al. showed the influence of the P141L mutation in Padron [140]. Exchange of the rigid proline residue with leucine altered the backbone organization at the respective position, causing the side chain of the adjacent Ser142 to move closer to the chromophore (fig. 4.2b). This was suggested to prevent His193 reorganization during cis-trans isomerization of the chromophore, which in turn prevented rotation of Arg66. Position 141 was reverted to proline in both the new variants as well as Kohinoor, and it was found to increase apparent brightness in Padron 1.9 during screening (fig. 3.5a). Brakemann et al. also suggested His193 to play a central role in the $\mathrm{pH}$ response of Padron and to interact with the on-state chromophore via $\pi$-stacking [140, 142], which could explain the observed change in brightness associated with L141P. Interestingly, replacement of His193 with glutamine resulted in an altered conformation and Glu211 interaction of Arg66 in Phanta, a weakly fluorescent protein with low quantum yield, although position 69 was occupied by a valine [192]. This highlights the important role of His193 and its surrounding amino acids. Altering its dynamics with L141P likely influenced this function, and presumably at least in part was responsible for the altered $\mathrm{pH}$ response of the new variants (tab. 3.7). A weakened $\pi$-stacking and altered protonation equilibrium in the on-state, as it was visible in the absorption spectra (fig. 3.16), could also contribute to more efficient off-switching, which was crucial for the new Padron variants (fig. 3.9). In addition, Ser142 stabilizes the chromophore by forming a hydrogen bond with the hydroxybenzylidene moiety in Padron, which in turn has direct contact with a water molecule situated in a pore of the $\beta$-barrel (fig. 4.3b). This latter interaction is absent in the on-state of Dronpa where the rotation of Ser142 causes the chromophore to move away from the pore [125]. Interestingly, reverting position 141 back to proline was responsible for a markedly differing off-state absorption spectrum in Padron0.9, a Padron variant of Padron with two mutations on the 
outside of the $\beta$-barrel that were introduced to facilitate crystallization and that was used by Brakemann et al. to elucidate the dynamics described above [140]. This variant displayed a pronounced absorption peak at $395 \mathrm{~nm}$ in the off-state trans-chromophore, reminiscent of the on-state absorption spectrum, and was fully switchable. Contrary to this, both the new variants and Kohinoor displayed no absorption peak of the protonated chromophore in the off-state despite a proline at position 141. This poses the question to which extent the findings described can be transferred to the new variants. With a total of 3 mutations in this region, namely R66K, A69V/I, and L141P, the dynamics described above were most likely heavily affected, and structural data could clarify their influence.
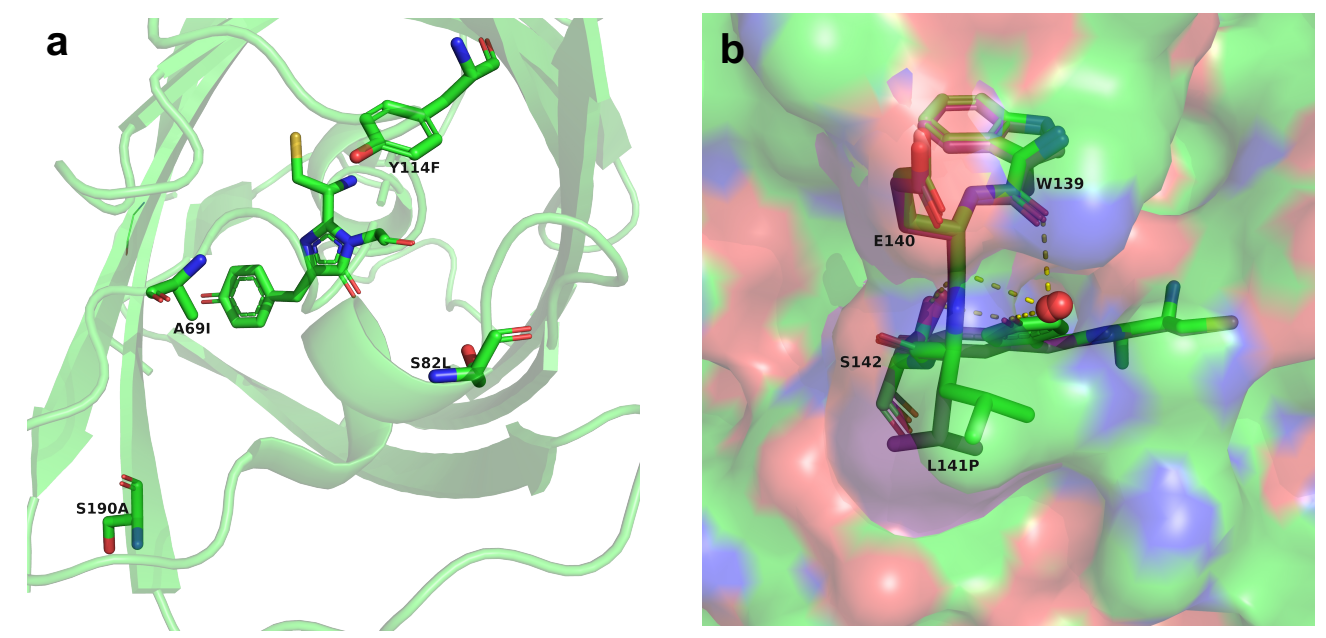

Figure 4.3: Mutated positions in Padron peripheral to the chromophore. (a) 3 amino acids at the bottom of the chromophore were mutated from polar side chains to hydrophobic ones. (b) Leu141 was mutated to proline. The position is part of a WEP motif common to many Anthozoan fluorescent proteins and is situated close to a water molecule (red sphere) that resides in an apparent pore of the barrel structure. Green and purple represent the on- and off-state of Padron, respectively (PDB IDs: 3ZUJ and 3ZUF, [61]).

\subsubsection{Influence of other positions}

Aside from the critical mutations discussed above, which rendered Padron 2.0 suitable for RESOLFT nanoscopy, the other mutations influenced protein characteristics and switching parameters, and possible explanations for their effects can be found in other publications, which will be referenced below. The first rounds of Padron mutagenesis were performed to increase switching speed of Padron in order to allow for shorter pixel dwell times during RESOLFT imaging. One mutation that contributed to this aim was F173S in pa22 (fig. 3.5b), a mutation which has been described in this context in a number of publications $[130,131,169]$. F173S was solely responsible for the introduction of switching in IrisFP [131], a photoconvertible protein based on EosFP [106] that can be reversibly switched between a fluorescent on- and a non-fluorescent off-state in both its green and red form. Adam etal. attributed the newly found switching capabilities to the smaller size of serine, allowing Met159 to adapt an altered conformation, which in turn increased the size 


\section{Discussion}

of the chromophore pocket allowing for cis-trans isomerization of the chromophore with rearrangements similar to Dronpa [125, 131, 139]. Similarly, the replacement of Met159 with the smaller amino acid threonine resulted in the generation of a Dronpa variant with faster switching kinetics [125]. These observations were used to generate a switchable version of Dendra2 [130]. Contrary to the initial assumption of the authors, a combination of F173L and M159A did not result in any improvement of switching capabilities compared to M159A alone. In Padron, however, a tyrosine residue at the latter position enabled its positive switching mode by stabilizing the trans-conformation of the chromophore in the off-state [61, 120, 140]. Tyrosine has a considerably larger side chain than alanine or threonine, therefore F173S presumably contributed to faster switching kinetics by enlarging the chromophore cavity. In addition, the replacement of phenylalanine with serine increased polarity at this position, and serine is capable of forming hydrogen bonds, which might have resulted in further stabilization of the trans-chromophore.

Similar assumptions about the cavity size can be made about the mutation T58S which was introduced with pa31 and had an effect on the switching half-time similar to the one of F173S. However, the removal of one methyl group facilitated by the change from threonine to serine could have enlarged the chromophore cavity only to a lesser extent than F173S. In addition, the methyl group of threonine faced away from the chromophore in the crystal structure of Padron [61, 140], which albeit could mean that its removal allowed for a new potential conformation of the hydroxy group of serine. Furthermore, Faro et al. showed that switching in Padron crystals at low temperatures was accompanied by motion of Met59, which is situated above the chromophore and in the vicinity of Thr58 as well as Ile195, whose side chain is located close to Thr58 [61]. Less steric hindrance due to a smaller serine side chain at position 58 could favor these motions. Ile195 has also been proposed to play a role in proton dynamics of the on-state of Dronpa by means of a water molecule [142]. Interestingly, Met59 was mutated to leucine in Padron 2.1. Because this variant originated from a directed evolution approach and displayed 3 new mutations it cannot be stated which effect a single of these mutations had on the switching characteristics. The lower brightness of this variant, however, suggests the influence of M59L on an potentially accompanying increased flexibility of the chromophore.

Faro et al. also stated that molecular dynamics simulations of Padron revealed that during on-switching, the hydrogen bonding of the chromophore, which stabilizes the off-state, is broken by means of backbone dynamics [61]. These dynamics, which were suggested to contribute to non-fluorescence of this state in the publication, might have been influenced by the mutation M40V: Met40 is part of a $\beta$-strand of the barrel on the side opposite to the chromophore cavity, and its side chain points towards the thiol group of the chromophore, which is built from the tripeptide CYG [120]. Replacement with the smaller valine side chain could increase chromophore flexibility by allowing greater movement towards this direction. Albeit, M40V increased brightness in the on-state of pa87 and resulted in a lower residual off-state fluorescence intensity.

K145N, a mutation of Padron 2.1, was reported to induce tetramerization in PDM1-4, 
a variant of Dronpa that differs only by the replacement of lysine by asparagine at this position [193]. Consequently, this mutation is likely responsible for the strong oligomerization observed in Padron 2.1 (fig. 3.21). The same effect was also observed during the generation of mIrisFP where K145I increased oligomerization [169]. According to Nguyen Bich et al., this tetramerization was favored by the smaller size of asparagine at position 145, which was less flexible than lysine. It was additionally stabilized by Tyr188 and Asp156 in PDM1-4, which in turn enabled interaction between monomers via hydrogen bonding of Asn145 with Asn158 due to the more rigid configuration [194]. This interaction of amino acids would be possible in Padron 2.1 as well because the involving amino acids are identical. Formation of the interaction described is thus most likely responsible for the high tendency of Padron 2.1 to oligomerize. In IrisFP, the additional mutation of Tyr189 to alanine (corresponding to Y188A in Padron and Dronpa) could revert the oligomerization tendency [169]; introducing this mutation into Padron 2.1 would probably have the same effect. Moreover, N158E and Y188A could break oligomerization tendency of pcDronpa, a photoconvertible version of Dronpa [182].

This interaction was also supposed to affect switching in PDM1-4 [193, 194]. The $\beta$-sheets of the AC interface, where Lys145 and Asn156 are located, were described to be flexible in the off-state of Dronpa [142, 143], and a more rigid organization was supposed to be responsible for the disability of 22G, the precursor of Dronpa [119], to switch [142]. Likewise, Padron 2.1 displayed slower switching kinetics for the activation in comparison to Padron 2.0. This difference, however, is in contrast to PDM1-4, where the more rigid interface resulted in slower off-switching kinetics [193]; these were accelerated in Padron 2.1 in comparison to Padron 2.0. However, the observations made in Dronpa and PDM1-4 do not necessarily transfer to Padron 2.1 because the switching regime is fundamentally different, with the former proteins displaying a negative and the latter a positive switching mode. In addition, M59L and F173S in Padron 2.1 might have compensated any effects that oligomerization might have had on the kinetic properties. The AC interface most likely has also been influenced by the E218G mutation that is present in the new Padron variants as well as Kohinoor, and the addition of GFPends. Gly218 was found to play a role in this interface in $22 \mathrm{G}$ as it is part of the C-terminal end, stabilizing the interaction of monomers [142]. Its mutation to glutamate was proposed to weaken this interaction by means of a repulsive effect towards Glu140 [142]. Indeed, G218E was a necessary mutation to remove the obligate tetramer formation of $22 \mathrm{G}$ in Dronpa, along with N102I [119, 142, 182]. Interestingly, both mutations were reverted in Kohinoor [121], whereas only position 218 was reverted to glycine in the new Padron variants. However, these dynamics were likely influenced in the new Padron variants by further mutations such as R221G, which is likewise located in the C-terminus, and S190A, with Ser190 being in contact with His216 close to the C-terminus via hydrogen bonds mediated by a water molecule. Interestingly, Ser190 was mutated to aspartate in Kohinoor. The prolonged termini as a result of GFPend insertion most likely affected the involvement of the C-terminus in the $\mathrm{AC}$ interface as well.

The addition of GFPends to both the $\mathrm{N}$ - and C-terminus was originally performed by 


\section{Discussion}

Shaner etal. during the generation of mCherry [98] from mRFP1 [97] to improve tagging performance, which was described as being unproblematic with GFP by the authors. Willig et al. added GFPends and the mutation E218G to Padron to improve mammalian expression in 2011 [195]. The same GFPends were added to generate pa57, which displayed no obvious changes with regards to its switching capabilities. Although the direct effect of GFPends on tagging performance of Padron was not addressed in this thesis, the new Padron variants showed improved labeling of cellular structures. The observation that the addition of GFPends could rescue fluorescence of Kohinoor that was otherwise lost during seminative PAGE (fig. 7.7) suggested an additional contribution of GFPends to stability of the proteins at unfavorable environmental conditions like the presence of a detergent.

In conclusion, a large portion of the positions mutated to create Padron 1.9, 2.0, and 2.1 have been described to be involved in critical properties of other fluorescent proteins from Anthozoa. These involvements were directly associated with either the dynamics observed during switching in Dronpa or IrisFP (Thr58, Arg66, Ala69, Leu141), or with the interaction of monomers during oligomerization (Glu218, GFPends). Other positions like Met40, Ser190, or Arg221 where in close vicinity to amino acids described to have an influence on the properties of fluorescent proteins. However, although the comparison of published data with the results presented in this thesis could explain the changes of protein characteristics observed in response to single mutations, all conclusions remain speculative. The majority of research referenced was conducted with negatively or non-switchable proteins, whereas Padron is a positively switching RSFP with distinct changes of the underlying mechanism [61, 140]. To validate the assumptions made here, further research aimed at the elucidation of mechanistic and structural events during switching of Padron 2.0 is needed. 4 of the 11 positions mutated in Padron had been altered in Kohinoor as well, 3 of those mutations were identical to the changes in Padron 2.0 (L141P, F173S, S190A/D, E218G). However, the resulting properties with regards to the switching performance was markedly different and will be discussed in the following sections.

\subsubsection{The bright intermediate state}

The initial focus on the on-switching speed during screening of mutants resulted in the generation of intermediate Padron variants such as pa69, which displayed a distinct and unusual switching behavior. While the activation curve of Padron has a shape similar to the graph shown in figure 4.4a and displays the expected exponential increase, the former variants showed a fast increase of fluorescence during on-switching with a following decay to a state of lower fluorescence (fig. 4.4b). This additional peak resulted in intricate behavior of samples in microscopy. Samples labeled with these variants quickly returned to the on-state during readout after deactivation, which was a problem for the establishment of RESOLFT nanoscopy. In addition, it was observed that images which were recorded with both the doughnut-shaped $405 \mathrm{~nm}$ and the readout $488 \mathrm{~nm}$ laser activated simultaneously displayed 
increased photon counts compared to images recorded with the readout laser alone (data not shown). Because this increase in signal could not be attributed to (auto)fluorescence of the sample in response to $405 \mathrm{~nm}$ excitation, it was reasoned that the additional counts originated from the bright intermediate state observed during screening, whose contribution to the fluorescence signal was higher than the initial loss during the first part of the activation curve (fig. 4.4).
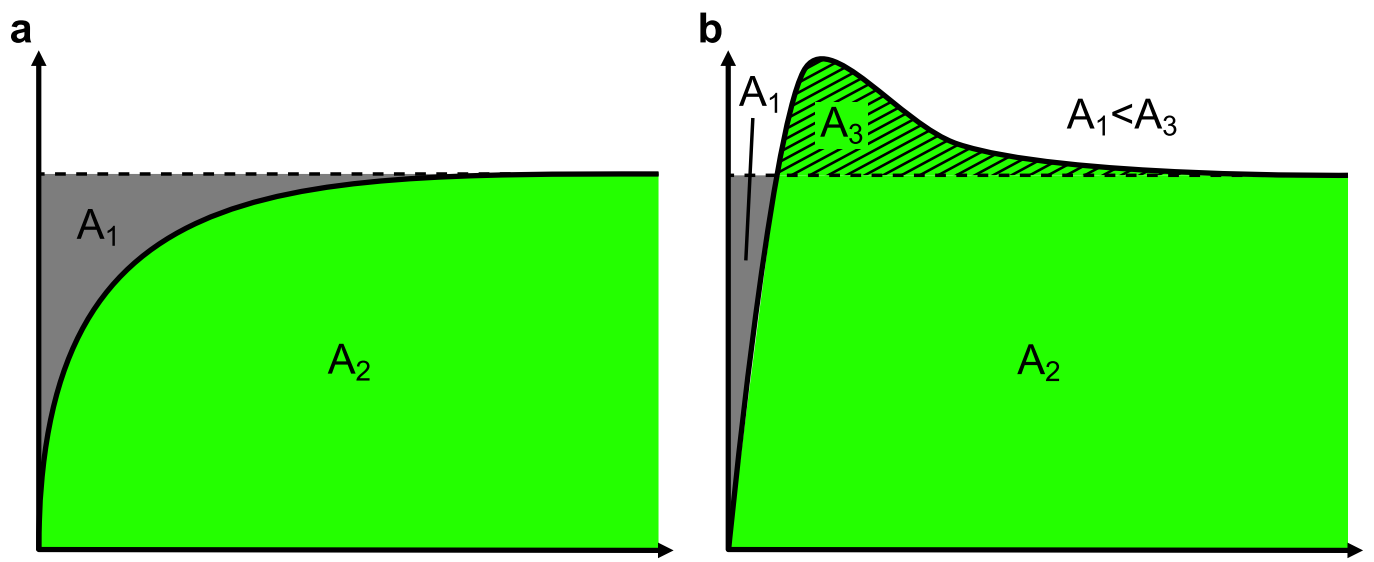

Figure 4.4: Fluorescence contribution of the bright intermediate state. (a) Onswitching curve of a positively switching RSFP such as Padron 2.0. The area under the curve represents total fluorescence recorded during the activation after off-switching. Without prior deactivation, the fluorescence of a fully activated RSFP recorded would amount to the sum of $A_{1}$ and $A_{2}$. (b) On-switching curve of a positively switching RSFP that switches on with a bright intermediate state, such as the intermediate variant pa69. The additional fluorescence $A_{3}$ is larger than the contribution of $A_{1}$, which is lost due to prior deactivation. The sum of $\mathrm{A}_{2}$ and $\mathrm{A}_{3}$ thus exceeds the fluorescence of an RSFP in the equilibrated full on-state $\left(\mathrm{A}_{1}\right.$ and $\mathrm{A}_{2}$ ).

This behavior had implications for potential RESOLFT imaging of variants displaying the bright intermediate state: In addition to the fast activation of peripheral fluorophores during readout, their maximal intensity exceeded the equilibrated on-state intensity from central fluorophores. Moreover, the bright intermediate state had a distinct bleaching behavior different from that of the on-state that influenced kinetics of the fluorophore, which additionally complicated establishment for RESOLFT nanoscopy with such variants.

A possible explanation for the effects described above might be found in the detailed switching mechanics of Padron, which were elucidated by Faro et al. by switching a Padron crystal at $100 \mathrm{~K}$ and employing molecular dynamics simulations [61]. They found that Padron, in contrast to Dronpa [125, 139], undergoes only minor structural changes upon switching from the non-fluorescent trans-state to the fluorescent cis-state. The first event was described to be the trans-cis isomerization to an intermediate state $\mathrm{I}_{c i s}$, which relaxed to a structurally identical state $\mathrm{B}_{\text {cis }}$ within minutes at $100 \mathrm{~K}$. Both states displayed similar absorption spectra with a blue-shifted peak for $\mathrm{I}_{c i s}$. A temperature increase above the glass transition temperature resulted in protonation of this state and a decrease of the deprotonated absorption peak in favor of an increased protonated peak [61]. 


\section{Discussion}

These events support the idea of a bright intermediate state. In addition, the shifted absorption peak of $\mathrm{I}_{c i s}$ is closer to the excitation wavelength of $488 \mathrm{~nm}$ used in both the automated screening microscope as well as the RESOLFT microscope. The following protonation resulted in a lower absorption at this wavelength, strongly decreasing excitation. Lastly, this protonation occurred concurrently with a blue-shift and broadening of the emission spectrum upon increasing the temperature above the glass transition temperature [61]. This altered emission spectrum was closer to the cutoff wavelength of the filters used in both setups. As multiple amino acids in the chromophore cavity were mutated in the process of generating the new Padron variants, the processes described were most likely influenced by this. Faro et al. described relaxation dynamics in the range of minutes at $100 \mathrm{~K}$, at room temperature the kinetics are expected to be much faster. The intermediate variants of Padron presumably allowed for the population of the bright intermediate state that was not seen in Padron at the intensities used due to slower kinetics of the processes following chromophore isomerization, which would be equivalent to a prolonged lifetime of the intermediate state. Higher intensities were observed to result in an even higher peak in the activation curve, which is in line with a more concerted population of this state.

With the R66K mutation in paA23, the bright intermediate state could not be observed with the intensities applied in the measurements performed for this thesis. This suggested either entirely different or faster dynamics of events following the trans-cis isomerization, which could be revealed by faster population of a putative intermediate bright state with higher laser intensities. Faro et al. stated that the minor structural changes, which do occur during the isomerization, take place exclusively above the chromophore [61]. The mutation of Arg66, which is situated below, possibly could have shifted these movements to its surroundings. The involvement of chromophore protonation in the bright intermediate state is supported by the interaction of Arg66 with His193, which was described to influence protonation [140, 142]. Because the bright intermediate state was unfavorable for RESOLFT applications, it was not analyzed in detail in this thesis, but intermediate variants presented here might constitute valuable candidates for further elucidation of the processes involved in the switching of Padron. In the final variants, however, this state was eradicated or at least strongly attenuated at the intensities applied for switching. This change resulted in switching characteristics that were suitable for RESOLFT nanoscopy.

\subsubsection{Improved protein and switching characteristics of the new Padron variants}

Screening of Padron variants was conducted at comparatively high laser intensities in the range of $\mathrm{kW} / \mathrm{cm}^{2}$ in order to reveal variants that performed well at these intensities and allowed for fast switching during RESOLFT nanoscopy. However, absolute relations between laser intensities applied and switching characteristics obtained from bacterial colonies measured at the automated screening microscope could not be directly transferred to the RESOLFT microscope due to inherent technical differences between the microscopes and samples: While the RESOLFT microscope constituted a confocal setup and samples were 
live HeLa cells measured at the interface between cell and a glass coverslip, bacterial colonies were an opaque, light-scattering sample, and the automated screening microscope had only a semi-confocal configuration with a pinhole size of multiple Airy units. Nevertheless, based on repeated empirical observations, the improvements of switching characteristics revealed at the latter microscope transferred well to RESOLFT applications. In this regard, the new Padron variants constituted a considerable improvement over Padron and Kohinoor.

Initial steps in the mutagenesis of Padron were aimed at improving activation speed to accelerate RESOLFT nanoscopy with shorter pixel dwell times. Unfortunately, this resulted in the generation of variants that displayed the bright intermediate state during activation. Variants following pa69 therefore were selected by favoring off-switching performance over activation speed. Still, half-times of the new Padron variants were approximately twice as fast compared to Padron, and on par with the performance of Kohinoor at lower $\mathrm{kW} / \mathrm{cm}^{2}$ intensities. At higher intensities the activation of Kohinoor was faster than in the new Padron variants, suggesting a non-linear light response to the $488 \mathrm{~nm}$ intensity. Likewise, switching to $95 \%$ of the full on-state required more time for Kohinoor at low and less time at high intensities.

Favoring deactivation over activation during later screenings resulted in variants which displayed a high propensity to switch to the off-state upon irradiation with $405 \mathrm{~nm}$. This property was significantly higher in the new Padron variants than in the original Padron and Kohinoor. The altered absorption spectra of the new Padron variants displayed an increase of the protonated absorption peak in the UV range of the on-state, explaining their increased tendency to absorb in this range and switch to the off-state. Conversely, Kohinoor showed a similar change of the absorption spectrum in the on-state, but switching to the off-state in E. coli was slow despite an increased protonated absorption peak. However, the kinetics of switching are also determined by the switching quantum yield, which was not addressed in this thesis and could explain the different kinetics observed for the new variants and Kohinoor. In addition, the apparent kinetic is influenced by the lifetimes of possible intermediate states and thus cannot be explained solely by altered absorption spectra [81].

The achievable residual off-state fluorescence intensity values of Kohinoor in bacterial colonies were even higher than in the original Padron. This was in contrast to in vitro switching, where Kohinoor reached similar off-state values as the new Padron variants. However, the intensities applied in vitro were much lower than the intensities applied in the automated screening microscope and those typically used in RESOLFT nanoscopy [124], supporting the idea of a non-linear dependency from the $405 \mathrm{~nm}$ intensity applied. One possible explanation for this effect could be the difference of RSFP concentrations between samples. While in vitro switching analysis was performed at approximately $20 \mu \mathrm{M}$, a range where PDM1-4 and Dronpa transition from monomer to dimer or tetramer, respectively [193], concentrations were expected to be in the $\mathrm{mM}$ range within E. coli cells [196].

In E. coli, high $405 \mathrm{~nm}$ intensities were detrimental for Padron and Kohinoor during offswitching, resulting in an increase of the residual fluorescence at higher doses, suggesting that $405 \mathrm{~nm}$ irradiation at these intensities induced photobleaching. This behavior restricts 


\section{Discussion}

the applicability of Padron and Kohinoor in RESOLFT nanoscopy as it limits the acceleration of pixel dwell times with increased laser intensities. It would also complicate the establishment of RESOLFT nanoscopy itself due to the non-linear correlation of achieved residual fluorescence and the dose of $405 \mathrm{~nm}$ light applied. It is to be expected that the effective light intensity that switches the chromophores in RESOLFT nanoscopy is higher than in opaque samples at the automated screening microscope.

Conversely, no negative effect of $405 \mathrm{~nm}$ irradiation could be seen in the new variants, and Padron 2.0 could sustain even prolonged irradiation, making it less sensitive to suboptimal imaging parameters. A possible explanation for the differences observed in this aspect between the new variants and Kohinoor or Padron could be altered kinetics for the protonation of the chromophore after isomerization: Upon switching to the off-state, the fluorescence peak of the protonated form vanishes with a concomitant increase of the absorption peak of the deprotonated form. If this deprotonation occurs much slower in Padron and Kohinoor, they remain sensitive to $405 \mathrm{~nm}$ excitation for a longer time after switching, which would result in photobleaching.

This improved $405 \mathrm{~nm}$ response most likely also benefited the new Padron variants in the analysis of switching fatigue, which was analyzed by repeated switching between $5 \%$ residual fluorescence and $95 \%$ of the full on-state. In addition, due to the fast switching of these variants, the irradiation duration of a single cycle was short in comparison to Padron and Kohinoor. Andresen et al. stated that the bleaching in Padron during repeated cycling mainly originated from fluorescence excitation and did not differ from photobleaching of the onstate [120]. Photobleaching in response to fluorescence excitation during repeated switching between saturated states is a considerable factor for positively switching RSFPs, because the majority of RSFPs in an ensemble are quickly rendered sensitive to their excitation wavelength (fig. 4.5). In the data presented here, the on-states of Kohinoor and Padron were more susceptible to photobleaching than Padron 2.0 and 2.1 (fig. 3.13), and both Kohinoor and Padron needed longer irradiation to be activated as well as to be switched off (figs. 3.9 and 3.10), which also added $405 \mathrm{~nm}$ induced photodamage. These factors combined resulted in the low performance observed in the analysis of switching fatigue, while Padron 2.0 could perform almost 1000 cycles before it was bleached to $50 \%$. This value is comparable to the performance of rsEGFP and amounts to about $50 \%$ of the cycles rsEGFP2 could undergo; albeit, the latter proteins were characterized in live Ptk2 cells expressing vimentin fusion constructs $[123,124]$. However, rsEGFP and rsEGFP2 are negatively switching RSFPs, which leaves Padron 2.0 as the best performing positively switching RSFP published so far.

The new Padron variants also show an activation process in the form of an initial increase in maximal fluorescence intensity during repeated switching. This was also found in rsCherryRev, a negatively switching variant of rsCherry [122]. The authors attributed this effect to structural rearrangements in response to switching and found that it was only present in variants that had a cysteine residue positioned opposite of the chromophore. No statement was made about the molecular mechanism, but as the final padron variants had no additional cysteine, this effect is probably not tied to the physical properties of cysteine itself. If such 

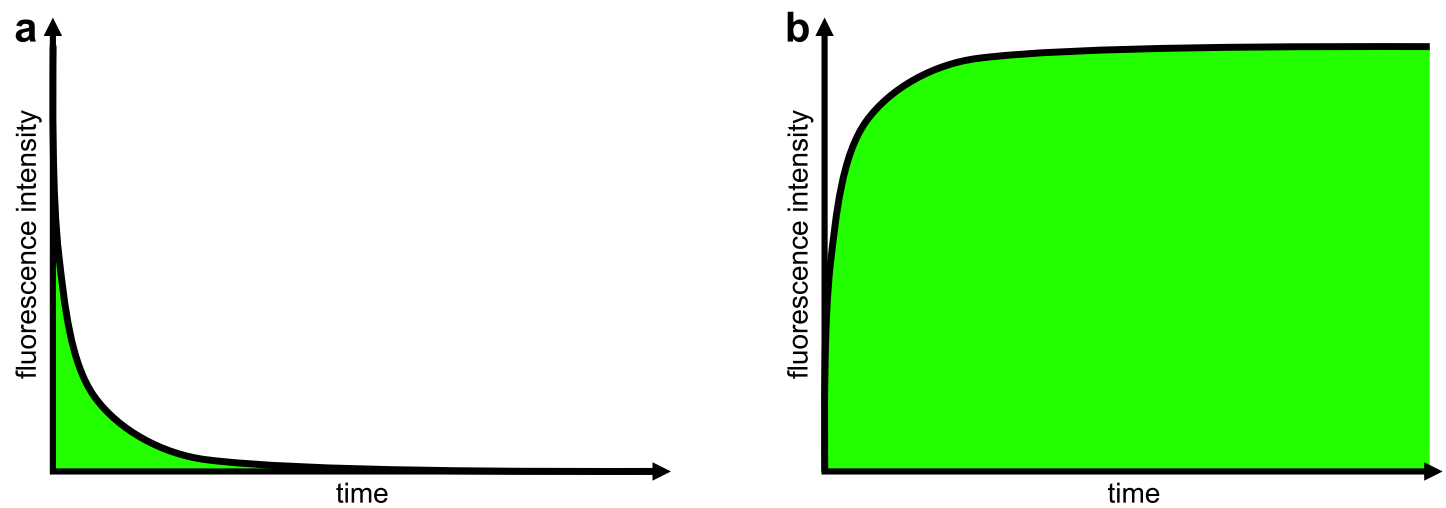

Figure 4.5: Fluorescence excitation during switching. Positively and negatively switching RSFPs in an ensemble fluoresce to a different extent while they are switched with their respective excitation wavelength. (a) Off-switching curve of a negatively switchable RSFP. Due to the exponential decay, the amount of RSFPs in the on-state quickly decreases during off-switching so that only a relatively small population of the ensemble is susceptible to photobleaching during saturation. (b) On-switching curve of a positively switchable RSFP. Due to the exponential increase of fluorescent RSFPs during on-switching, a relatively large population is susceptible to photobleaching during saturation.

structural rearrangements in response to switching exist, however, they could explain the different equilibrium state fluorescence intensities found in Padron 2.1, which resulted from different on-state intensities depending on the sequential order of on- and off-switching in vitro.

Aside from switching improvements, the spectral properties of the new padron variants were considerably altered. Lower absorption coefficients at the maximum of the deprotonated states, which albeit were blue-shifted and thus closer to the $488 \mathrm{~nm}$ excitation laser in the RESOLFT microscope, and a lower quantum yield in all variants resulted in impaired molecular brightness. This was likely influenced by the lower acid tolerance of padron variants, which in turn displayed a better tolerance of high $\mathrm{pH}$ values above 8.5 than Padron. However, the pH-dependency was only analyzed for the ground state, so that an influence of the switching state in these measurements cannot be excluded, although the on-state $\mathrm{p} K_{a}$ value of Padron0.9 was published to be 6.0 [140], which is in line with 5.9 for Padron.

4 of the 11 mutations introduced into Padron 2.0, namely L141P, S190D, E218G and R221G, had side chains pointing to the outside of the $\beta$-barrel. Although E218G was described to be in part responsible for the tetramerization of $22 \mathrm{G}$ [142], Padron 2.0 was found to be a monomer at $6^{\circ} \mathrm{C}$ and $10 \mu \mathrm{M}$, which makes it the Padron variant with the least propensity to oligomerize. In contrast, Kohinoor was found to dimerize under these conditions. Of the 7 mutations that distinguish Kohinoor from Padron, 6 had side chains on the outside of the $\beta$-barrel, with two of them being N102I and E218G. Asparagine and glutamate at these positions were sufficient to break tetramerization in Dronpa [142], and the mutations at these two positions are likely responsible for the dimerization tendency of Kohinoor. The 4 residual mutations on the exterior likely attenuated the oligomerization tendency. While 


\section{Discussion}

Padron formed stable tetramers, Kohinoor displayed fast equilibrium formation of the dimer, suggesting that the mutations of residues pointing to the outside of the structure could potentially lead to improved monomeric behavior in other variants. Padron 2.0 could benefit from this observation because high concentrations still resulted in the formation of tetramers. In conclusion, Padron 2.0 displayed minor drawbacks with regards to brightness or its oligomerization, but was the best performing Padron variant analyzed in this thesis. Especially its improved switching fatigue and off-switching efficiency were improved, two critical parameters for the establishment of RESOLFT nanoscopy with this RSFP. In addition, its improved expression and maturation rendered it suitable for live-cell applications. Padron 1.9 and 2.1 shared many of the improvements, but showed some additional drawbacks in comparison with Padron 2.0.

\subsubsection{Padron 1.9 and 2.1}

Padron 1.9, 2.0, and 2.1 had very similar spectral characteristics, and switching parameters such as the residual off-state fluorescence were comparable to Padron 2.0. Certain key aspects, however, differed considerably between variants. Padron 1.9 on the one hand was brighter than Padron 2.0 and displayed better tolerance of low $\mathrm{pH}$ values, but was more sensitive to photobleaching and switching fatigue. Therefore, it would be the better candidate for scenarios in which a bright positively switching RSFP with a limited number of switching cycles is needed, such as single image RESOLFT microscopy of sparsely labeled structures.

In contrast, finding a suitable application for Padron 2.1 was more problematic due to its drawbacks. While the lowered brightness compared to Padron 2.0 was compensated by lower residual fluorescence and better switching fatigue performance, the markedly higher propensity to oligomerize could be a challenge in applications where dynamic interactions are of interest. In addition, the determination of spectral properties suffered from low reproducibility, which is visible in the high standard deviation of the extinction coefficient of Padron 2.1: It amounted to about one third of the parameter value. If these difficulties were due to the oligomeric tendency of Padron 2.1 and slowly adapting equilibria, the elimination of a putative interface between monomers evoked by the mutation K145N could solve them. The feasibility of this was shown for IrisFP and pcDronpa [169, 182].

Because of these drawbacks, Padron 1.9 and 2.1 were not used to establish RESOLFT microscopy in favor of Padron 2.0, which was considered to be the variant with the most promising characteristics for this application. However, their switching characteristics suggest a good qualification for RESOLFT nanoscopy applications.

\subsubsection{RESOLFT nanoscopy with Padron 2.0}

RESOLFT nanoscopy with Padron 2.0 was demonstrated with 3 different approaches in HeLa cells transiently transfected with vimentin fusion constructs. While the vimentin structures displayed a tendency to build condensed, thickened filaments in response to overexpression of fusion constructs, STED imaging of the samples could prove their suitability for the es- 
tablishment of RESOLFT microscopy. With Padron 2.0, STED imaging revealed a minimal filament size below a FWHM of $50 \mathrm{~nm}$. Similarly, RESOLFT imaging with sequential activation, deactivation, and readout could achieve a FWHM of $58 \mathrm{~nm}$. This value was higher than, but comparable to, 45-48 nm achieved with keratin19-rsEGFP2 fusion constructs [124] at similar pixel dwell times. RESOLFT images with rsEGFP as a fusion label could achieve a resolution as low as $36 \mathrm{~nm}$, but required pixel dwell times of up to $22 \mathrm{~ms}$ [123]. However, imaging with a positively switching RSFP has advantages over negatively switching RSFPs. If sub-diffraction resolution is only needed at certain time points during time-lapse imaging, Padron 2.0 can be imaged without any activation step, which in contrast is necessary for negatively switching RSFPs and would increase the amount of light the sample is exposed to. Naturally, this also applies to STED imaging with Padron, where omitting the activation step could further accelerate image acquisition.

Compared to sequential switching, the second approach in which the $405 \mathrm{~nm}$ doughnut was kept active during the readout step displayed a higher minimal FWHM of $68 \mathrm{~nm}$, but overall resolution was comparable to the first sequential approach. The FWHM values of around $80 \mathrm{~nm}$ were also in line with the resolution claimed by Tiwari et al. for RESOLFT nanoscopy with Kohinoor in a similar approach [121]. However, the image presented in their study displays severe loss of fluorescence signal in dimmer filaments (fig. 1.10).

Finally, the third RESOLFT approach in this thesis simplified the switching scheme by omitting the activation step, establishing one-step RESOLFT. This employed the hypothesized advantage of positively switching RSFPs for RESOLFT microscopy. Negatively switching RSFPs limit the photon budget in RESOLFT imaging because the readout wavelength also switches the fluorophore to the off-state. For dim structures, this requires multiple switching cycles to increase the photon budget by accumulating multiple pixel dwell times. With one-step RESOLFT, it could be shown that ensemble suppression of peripheral fluorophores with a $405 \mathrm{~nm}$ doughnut was indeed possible, achieving lateral resolutions for single filaments of $74 \mathrm{~nm}$. The decisive characteristic which allowed this mode of RESOLFT nanoscopy was the improved off-switching rate $\mathrm{k}_{\text {off }}$ of Padron 2.0 (fig. 4.6). If $\mathrm{k}_{\mathrm{off}}$ is lower than the rate for on-switching $\mathrm{k}_{\mathrm{on}}$, the activation of fluorophores cannot be compensated by off-switching with $405 \mathrm{~nm}$ (fig. 4.6d). Conversely, if $\mathrm{k}_{\text {off }}$ is faster than $\mathrm{k}_{\mathrm{on}}$, simultaneous irradiation with a $405 \mathrm{~nm}$ doughnut and a Gaussian-shaped $488 \mathrm{~nm}$ readout laser can suppress the ensemble activation of peripheral fluorophores, confining the fluorescence excitation volume to a volume that is smaller than the diffraction limit (fig. 4.6c). This effect is further promoted by different spatial distribution of the doughnut and the Gaussian-shaped beam peak intensities: The circular maximal fluorescence of the doughnut is aligned with the sides of the Gaussian spot (fig. 4.6c, d).

Confinement of fluorescence excitation to the central region is achieved by two entangled mechanisms. Naturally, other non-radiative decay processes than those described below apply, but will be ignored for the following contemplation. The $405 \mathrm{~nm}$ doughnut switches the majority of fluorophores to the non-fluorescent off-state, from which $488 \mathrm{~nm}$ excitation does not result in fluorescence emission, but rather switches the RSFP back to the on-state. 
a
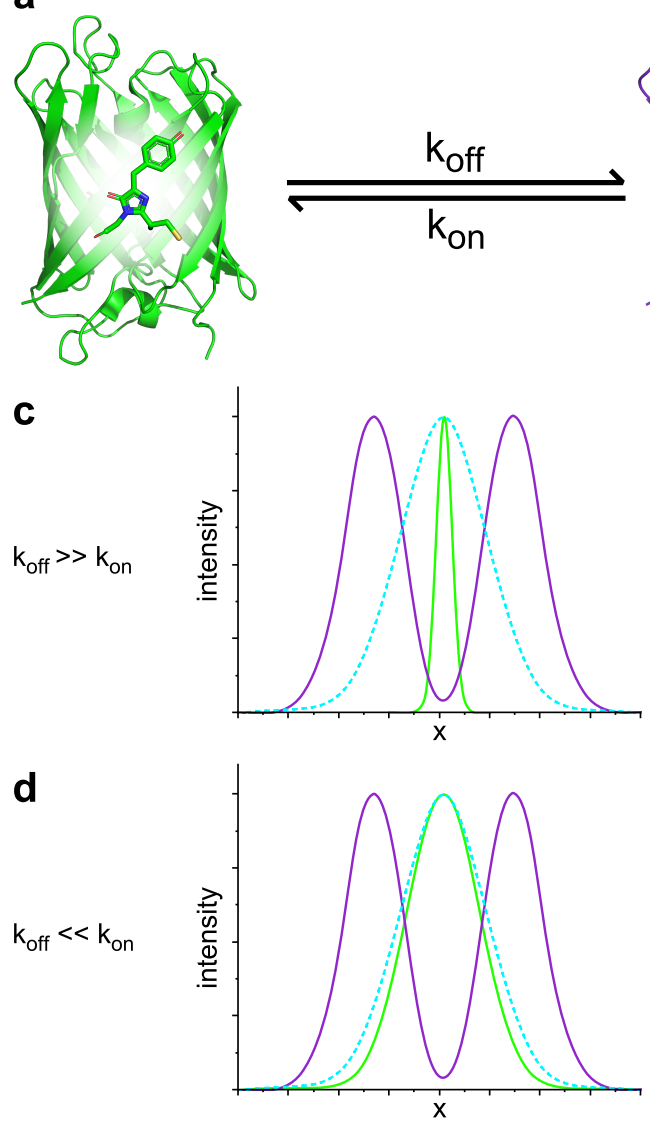

b
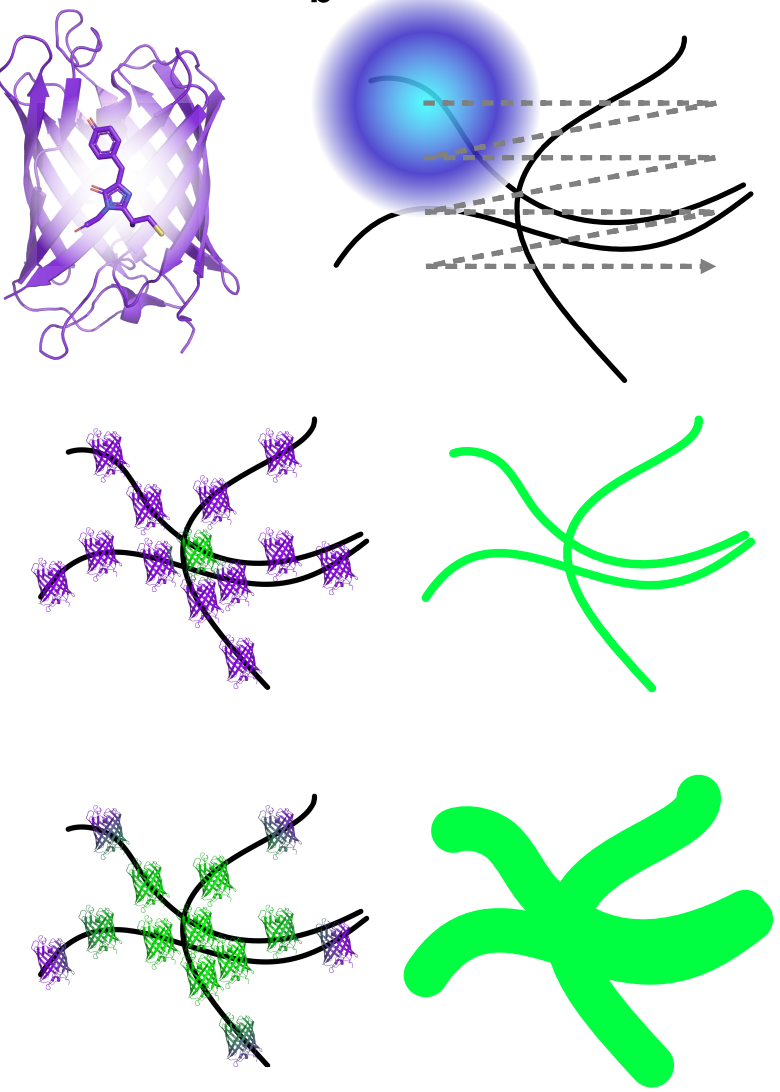

Figure 4.6: Theoretical basis of one-step RESOLFT. (a) Padron variants displayed different rates for switching to the off-state $\left(\mathrm{k}_{\text {off }}\right.$ rate constant) and to the on-state $\left(\mathrm{k}_{\mathrm{on}}\right.$ rate constant). (b) During one-step RESOLFT imaging both the $405 \mathrm{~nm}$ doughnut and the $488 \mathrm{~nm}$ Gaussian-shaped laser were active for the complete dwell time at all coordinates. (c) With a rate constant $\mathrm{k}_{\text {off }}$ much larger than $\mathrm{k}_{\mathrm{on}}$ the off-switching doughnut (purple graph) was dominant in comparison to the on-switching readout laser (blue graph). This resulted in a smaller effective PSF for fluorescence excitation confining the source of fluorescence to nanoscale dimensions. (d) With a rate constant $\mathrm{k}_{\text {off }}$ much smaller than $\mathrm{k}_{\text {on }}$ activation of fluorophores cannot be compensated by off-switching, resulting an effective PSF for fluorescence excitation that barely differs from diffraction-limited applications.

Here, a single fluorophore can absorb a photon with a wavelength of $405 \mathrm{~nm}$, resulting in off-switching, or one with a wavelength of $488 \mathrm{~nm}$, resulting in fluorescence emission. Which of these events occurs is a stochastic process and can thus be steered by the intensity ratio of deactivation and readout laser, reminiscent of the principle of STED [40, 41]. Therefore, under the assumption that during fluorescence readout a steady-state of fluorophores in the off- and on-state has formed, the achievable resolution is in theory regulated only by the ratio of $405 \mathrm{~nm}$ and $488 \mathrm{~nm}$ light intensities for Padron 2.0 in one-step RESOLFT nanoscopy and allows for arbitrary readout duration. In contrast, the sequential approach regulates the confinement of the excitation volume via both doughnut intensity and duration, and readout is limited by activation of peripheral fluorophores. 
However, in practical applications, certain limitations apply. The confinement of active fluorophores is limited by the switching background. If the sub-diffraction sized excited spot is very small, its contribution to the pixel photon counts can be masked by active fluorophores from the periphery, resulting in a diffraction-limited fluorescence readout. For rsEGFP2, the residual fluorescence was reported to be approximately $10 \%$ in eukaryotic cells [124]. While Padron 2.0 could achieve a switching background below $1 \%$ in E. coli, the residual fluorescence in eukaryotic cells is unknown and needs to be addressed in further studies. Another factor in the one-step RESOLFT approach is $405 \mathrm{~nm}$ induced fluorescence. In the case of Padron 2.0, it results from both autofluorescence of the cell as well as Padron 2.0 itself, which displays weak fluorescence emission in response to $405 \mathrm{~nm}$ excitation. Nevertheless, high $405 \mathrm{~nm}$ intensities and a small fluorescence contribution of central fluorophores could potentially result in a relevant photon count from $405 \mathrm{~nm}$ induced fluorescence. This could potentially be overcome by FLIM, but the fluorescence lifetime of intracellular molecules like NADH and porphyrin or GFP has been shown to depend on the tissue or the viability of the cell, respectively [4, 197]. Consequently, the applicability of FLIM has to be evaluated for each respective experiment due to the multitude of factors influencing fluorescence lifetime [198].

After all, high $405 \mathrm{~nm}$ intensities could also result in severe photodamage to the cell [177, 199]. Wäldchen etal. analyzed the influence of prolonged illumination as it occurs in PALM experiments on cellular viability. For $405 \mathrm{~nm}$ they found that at an intensity of $0.02 \mathrm{~kW} / \mathrm{cm}^{2}$ illumination of the whole cell for 60 seconds amounting to $1200 \mathrm{~J} / \mathrm{cm}^{2}$ was sufficient to kill all U2OS cells irradiated with a continuous wave laser. Other findings presented were: less phototoxicity of continuous wave laser irradiation compared to pulsed irradiation, a higher resistance of HeLa cells compared to U2OS cells, better tolerance of light at $37^{\circ} \mathrm{C}$, and increased damage in the presence of fluorophores. Lowering the $405 \mathrm{~nm}$ dose to $480 \mathrm{~J} / \mathrm{cm}^{2}$ resulted in cell death of $14 \pm 20 \%$ of the cells, while $48 \mathrm{~J} / \mathrm{cm}^{2}$ killed $3 \pm 8 \%$. The latter value was thus recommended as the uncritical limit [177]. While $405 \mathrm{~nm}$ exposure for the activation step of rsEGFP2 is relatively short and does not exceed this value [124, 177], the deactivation step for RESOLFT images presented here was in the range of $57-93 \mathrm{~J} / \mathrm{cm}^{2}$ (cf. tab. $5.10 \mathrm{in}$ the methods section). This range exceeded the recommended dose of $48 \mathrm{~J} / \mathrm{cm}^{2}$ by a factor of approximately 2. Considering the effects observed at $480 \mathrm{~J} / \mathrm{cm}^{2}$ by the authors, the values used here were expected to cause only minor phototoxic effects. Indeed, no phototoxic effects were observed when images were recorded for this thesis, but viability of imaged cells was not addressed with a dedicated experiment. In addition, Wäldchen et al. performed their experiments under constant or pulsed illumination of whole cells. In contrast, RESOLFT nanoscopy as a coordinate-targeted point scanning method limits the irradiation to a small volume that is moved through the cell. The spatial and temporal distribution of irradiation within the cell could result in a different response, giving the cell more time to cope with phototoxic effects. Padron 2.1 displayed more efficient switching to the off-state and could potentially reach $405 \mathrm{~nm}$ doses below $48 \mathrm{~J} / \mathrm{cm}^{2}$.

For the different RESOLFT approaches shown here, $405 \mathrm{~nm}$ doses did not differ fundamentally. As Padron 2.0 could be shown to withstand prolonged $405 \mathrm{~nm}$ irradiation without 


\section{Discussion}

any influence on the switching background, it can be assumed that the lower bleaching of Padron 2.0 during one-step RESOLFT in comparison with sequential switching resulted from the omission of the activation step.

Another possibility to reduce phototoxic effects could be the establishment of two-photonillumination. It was shown that Kohinoor can be excited with a wavelength of $900 \mathrm{~nm}$, and Dronpa-M159T [125] could be switched to the on-state via two-photon absorption [200]. Whether these observations can be applied to Padron 2.0 is unknown, but the employment of two-photon switching in both states would solve not only $405 \mathrm{~nm}$ induced phototoxicity but also optical problems occurring with the use of this wavelength. A higher wavelength allows for a deeper penetration depth in tissue, whereas aberrations of $405 \mathrm{~nm}$ result in both a misshaped doughnut as well as a spatial mismatch of readout and deactivation beam [14]. The RESOLFT images presented here display the potential of Padron 2.0 for nanoscopy and further studies can elaborate on this. The original Padron was demonstrated to facilitate three-color imaging with bsDronpa and rsFastLime [120], albeit with diffraction-limited microscopy. This approach, however, is not applicable for Padron 2.0 as it relies on the full deactivation of Padron with $405 \mathrm{~nm}$ and subsequent spectral separation of bsDronpa and rsFastLime. The latter two RSFPs display negative switching, Padron 2.0 deactivation with a $405 \mathrm{~nm}$ doughnut would thus transfer them to the fluorescent on-state and result in overlapping emission of all proteins, which could not be separated spectrally. This behavior excludes the combination of Padron 2.0 with negatively switching RSFPs with similar fluorescence excitation and emission. Red-shifted RSFPs on the other hand would allow for spectral separation. Recently, live-cell RESOLFT nanoscopy has been demonstrated with rsFusionRed3 [129]. This red-shifted negatively switching RSFP can be activated with $405 \mathrm{~nm}$ or $488 \mathrm{~nm}$, but requires fluorescence excitation with $592 \mathrm{~nm}$, which is well separated from the excitation and emission range of Padron 2.0. However, different doughnut-shaped off-switching lasers are required: Both rsFusionRed3 and Padron 2.0 can be deactivated simultaneously with a $405 \mathrm{~nm}$ and a $592 \mathrm{~nm}$ doughnut. Readout has to occur sequentially within the time frame of a single pixel as $488 \mathrm{~nm}$ readout of Padron would activate peripheral rsFusionRed3 fluorophores [129]. This would multiply the pixel dwell time. In contrast, a combination with a red-shifted positively switching RSFP could overcome this drawback, which has been demonstrated for negatively switching RSFPs in 2014 [154]. In 2008, Stiel etal. published rsCherry [122], but no RESOLFT applications have been presented so far. Off-switching of rsCherry was demonstrated with $445 \mathrm{~nm}$, whereas rsFusionRed3 activation was shown to be feasible with a wide range of wavelengths, namely $405 \mathrm{~nm}, 488 \mathrm{~nm}$, and $510 \mathrm{~nm}$ [129]. Ideally, a red-shifted positively switching RSFP that could be switched to the off-state with $405 \mathrm{~nm}$ with kinetics similar to Padron 2.0 would enable two-color RESOLFT imaging without prolonged pixel dwell times. Deactivation would occur for both fluorophores with the same doughnut, and readout could be facilitated simultaneously with two wavelengths and spectral separation. The use of FLIM would allow the combination of spectrally identical proteins if the fluorescence lifetimes are sufficiently separated, which has been shown for a combination of rsEGFP and Dronpa [5]. The lifetimes of these proteins differ by $0.9 \mathrm{~ns}$, which is almost 
identical to the difference of $0.8 \mathrm{~ns}$ measured for Padron 2.0 and 2.1. Because both proteins display similar switching characteristics, they would constitute an ideal pair for RESOLFT nanoscopy with FLIM, which of course could be extended to three-color nanoscopy with a matching red-shifted positively switching RSFP.

Furthermore, the switching characteristics of Padron 2.0 and the other new variants render them promising candidates for the establishment of RESOLFT implementations different from the point-scanning approach used here. While NL SIM imaging was demonstrated with Kohinoor [161], the improved switching characteristics of the new variants are expected to enhance this application of a positively switchable RSFP. Moreover, the efficient off-switching kinetics are expected to result in good performance in protected STED measurements, where peripheral fluorophores are rendered unresponsive to the STED wavelength and display less bleaching than in standard STED imaging [174]. While one-step RESOLFT could be demonstrated with short pixel dwell times in this thesis allowing for fast pointscanning RESOLFT nanoscopy, the new variants would benefit from further acceleration with parallelized RESOLFT $[129,156,158,159]$. Alternatively, positively switchable RSFPs are particularly suited for smart RESOLFT [155]. In order accelerate imaging and to reduce the light dose, smart RESOLFT probes the fluorescence at every position. If no fluorescence is recorded during this probing step, the switching sequence is omitted and the scanner moves to the next position. Negatively switching RSFPs necessarily have to be switched with an additional wavelength in the probing step. Conversely, positively switchable RSFPs result in a further reduction of the light dose applied to the sample due to the nature of their switching regime.

\subsection{Conclusion and outlook}

In conclusion, one-step RESOLFT nanoscopy with Padron 2.0 could be shown as a fast implementation of a positively switching RSFP that matched pixel dwell times reported for rsEGFP2 [124]. This was made possible by the significantly improved off-switching efficiency, which allowed for suppression of activation and fluorescence emission under simultaneous irradiation with light of $405 \mathrm{~nm}$ and $488 \mathrm{~nm}$. One-step RESOLFT provides a simplified approach to RESOLFT nanoscopy in which the achievable resolution is defined by the intensity ratios of doughnut- and Gaussian-shaped beams.

It could also be shown that Padron 2.0 displays strongly improved switching characteristics in comparison to Kohinoor. The most critical differences were the efficient off-switching of Padron 2.0 under simultaneous $488 \mathrm{~nm}$ irradiation and its significantly higher switching fatigue performance. In both parameters, Kohinoor displayed only minor improvements in comparison to Padron. These are expected to be insufficient for its application in one-step RESOLFT microscopy.

Padron 1.9 is a bright positively switching RSFP suitable for applications where high fluorescence emission is more important than a high number of switching cycles, and Padron 2.1 displayed an increased off-switching performance in comparison to Padron 2.0. Potentially, 


\section{Discussion}

the advantages of Padron 1.9 and 2.1 could also be introduced to Padron 2.0 without the drawbacks of the former variants in further studies. Minor drawbacks like oligomerization of Padron 2.0 at high concentrations exist, but the comparison with Kohinoor and other FPs with high sequence identity has shown that there are multiple sites in the protein that could be addressed with additional mutageneses to improve this aspect.

Further improvements of Padron 2.0 should be aided by the analysis of structural changes induced by the mutations introduced so far to verify or falsify the theories discussed in section 4.2.3. At the moment, the structural mechanisms that are responsible for the improvements observed remain elusive. The analysis of the altered structural organization is expected to reveal distinct changes within the network of interactions present within the $\beta$-barrel. Further mutagenesis could elaborate on such findings, allowing for the generation of even better performing positively switchable RSFPs.

Moreover, future studies should focus on enhancing the range of applications. Padron 2.0 and 2.1 likely constitute suitable pair of candidates for two-color RESOLFT imaging with FLIM, which could be further extended with a red-shifted positively switching RSFP. Moreover, the implementation of faster RESOLFT nanoscopy methods should extend the range of applications for the new variants in the elucidation of live-cell dynamics. The images shown here provide a starting point for the imaging of more complex tissue samples or organisms. 


\section{Methods}

If not listed in the protocol, detailed concentrations of buffers, solutions, and media are listed in chapter 6. Primer sequences are listed in section 6.3.

\subsection{Protein expression}

\subsubsection{Bacterial expression systems}

\subsubsection{Plasmids}

pQE-31 expression vector Padron mutants up to variant pa69 were mutagenized in the pQE-31 vector (Qiagen, Hilden, Germany). pQE-31 is a $3.4 \mathrm{kbp}$ expression vector featuring the colE1 origin of replication and a $\beta$-lactamase gene for ampicillin resistance. Expression of the protein inserted into the multiple cloning site (MCS) is regulated by the T5 promoter and lac operator. A 6xHis-tag is added to the protein N-terminally. Padron variants were inserted into the MCS with restriction enzymes BamHI and HindIII (see section 5.2.1 for detailed protocols). Sequencing was done with either primer 298 (forward) or 299 (reverse).

pBAD expression vector Padron mutants following pa69 were expressed and mutagenized in the $\mathrm{pBAD} /$ HisB backbone of $\mathrm{pBAD}$-mKalama1, which was a gift from Robert Campbell (Addgene Plasmid \# 14892 [201]). Expression is controlled by the araBAD promoter and a $6 x H i s-t a g$ is added N-terminally to the protein inserted into the MCS. The vector also features a $\beta$-lactamase gene for ampicillin resistance. For comparative measurements, all Padron variants were cloned into this vector via polymerase chain reaction (PCR) amplification of the variant from the pQE-31 plasmid with primers 4027 and 4098 (variants without GFPends) or 2943 and 3048 (variants with GFPends). Padron variants were inserted into pBAD/HisB with restriction enzymes EcoRI and XhoI. Sequencing was done with either primer 1639 (forward) or 1640 (reverse). pBAD plasmids coding for DsRed, dTomato and mEGFP had previously been cloned in our group. DsRed originated from DsRed1-N1-pRSETa [202], dTomato from pRSETb-dTomato, which was a gift from Roger Tsien [98], and mEGFP had been created by site-directed mutagenesis from pRSETa-EGFP [202].

Kohinoor Kohinoor/pRSETb was a gift from Takeharu Nagai (Addgene plasmid \# 67770 [121]). Kohinoor was amplified from this vector via PCR with primers 4027 and 4098 followed by insertion into the pBAD/HisB vector with restriction enzymes EcoRI and XhoI.

\subsubsection{Bacterial strains}

DH5 $\alpha$ E. coli DH5 $\alpha$ bacteria were used for amplification of plasmids after cloning. This strain was originally bought as MAX Efficiency ${ }^{\circledR}$ DH $5 \alpha^{\mathrm{TM}}$ Competent Cells (Invitrogen, Carls- 


\section{Methods}

bad, CA, USA) and proliferated in lysogeny broth (LB-medium) in the lab. For all applications described in this thesis electrocompetent cells were prepared (cf. section 5.1.1.3).

SURE SURE competent cells (Stratagene, CA, USA) were used for expression of proteins from the pQE-31 vector. Untransformed SURE E. coli were grown in LB-medium into larger cultures from which electrocompetent aliquots were prepared (cf. section 5.1.1.3).

TOP10 E. coli TOP10 bacteria were used for expression of proteins cloned into the $\mathrm{pBAD} /$ HisB vector. This strain was originally bought as ready-to-use electrocompetent cells (One Shot ${ }^{\mathrm{TM}}$ TOP10 Electrocomp ${ }^{\mathrm{TM}}$ E. coli, Thermo Fisher Scientific, Waltham, MA, USA). For all applications described in this thesis, untransformed TOP10 E. coli were grown in LB-medium into larger cultures from which electrocompetent aliquots were prepared (cf. section 5.1.1.3).

\subsubsection{Transformation}

Bacterial cells were transformed via electroporation with a modified protocol based on a publication from Dower etal. [203].

Electrocompetency In order to prepare bacterial strains for electroporation, $500 \mathrm{ml}$ LBmedium were inoculated with $5 \mathrm{ml}$ of an overnight culture. The culture was grown to an $\mathrm{OD}_{600}$ value of $0.6-1.0$ and then cooled on ice for $15-30 \mathrm{~min}$. Cells were pelleted at $3079 \mathrm{rcf}$ and $4^{\circ} \mathrm{C}$ in a Sorvall RC6 Plus cooling centrifuge with HS-4 rotor (Thermo Fisher Scientific, Waltham, MA, USA). The cell pellet was resuspended once with $500 \mathrm{ml}$ sterile $\mathrm{H}_{2} \mathrm{O}$, once with $250 \mathrm{ml}$ sterile $\mathrm{H}_{2} \mathrm{O}$ and once with $10 \mathrm{ml} 10 \%$ glycerin, and cells were subsequently pelleted under the same conditions as before. Pellets were resuspended in $1 \mathrm{ml} 10 \%$ glycerin and stored as $50 \mathrm{\mu l}$ aliquots at $-80^{\circ} \mathrm{C}$.

Electrotransformation Aliquots of electrocompetent cells were thawed on ice and diluted with $70-270 \mu \mathrm{l}$ sterile water on ice for $1-5$ transfections, respectively. $1 \mu \mathrm{l}$ purified plasmid containing approximately 75-300 ng DNA, $5 \mu \mathrm{l}$ mutagenesis sample, or $5 \mu$ l ligation sample were mixed with $50 \mathrm{pl}$ diluted electrocompetent cells and transferred to an electroporation cuvette (Cell Projects, Herrietsham, UK). Electroporation was performed with a pulse controller set to $200 \Omega$ and a gene pulser set to $25 \mu \mathrm{F}$ (both BioRad, Hercules, CA, USA). Transformed samples were diluted in $1 \mathrm{ml} \mathrm{LB}$-medium and incubated for approximately $1 \mathrm{~h}$ at $37^{\circ} \mathrm{C}$ before LB-plates or LB liquid cultures were inoculated.

\subsubsection{Growth conditions}

Transformed E. coli were either used for inoculation of LB-plates on the same day or stored for up to 2 weeks at $4^{\circ} \mathrm{C}$. Inoculation was done with an inoculating loop (cloning, plasmid preparation, RSFP characterization) or by distributing diluted transformed E. coli on the whole 
plate (screenings). Temperature for bacterial growth was $30^{\circ} \mathrm{C}$ or $37^{\circ} \mathrm{C}$. LB-plates used for protein expression from the pBAD vector contained $0.02 \%(\mathrm{w} / \mathrm{v})$ arabinose, whereas expression from the pQE-31 vector was sufficient without induction. Detailed growth conditions such as incubation times and storage conditions prior to measurements are described in the respective methods section.

\subsubsection{Mammalian expression systems}

\subsubsection{Mammalian plasmids}

All coding sequences of fluorescent proteins were PCR amplified from the pBAD plasmids described in section 5.1.1.1. The respective primers that were used to amplify Padron variants and the restriction enzymes used for insertion into the expression plasmid are listed in table 5.1. Sequences of primers used for cloning are listed in table 6.3 in section 6.3, primers used for sequencing are listed in table 6.1 in the same section.

Table 5.1: Primers and restriction enzymes used for cloning of mammalian fusion constructs

\begin{tabular}{lccc}
\multicolumn{4}{c}{ Primer (forward, reverse) } \\
Fusion construct & Padron $\mathbf{1 . 9 , 2 . 0 , 2 . 1}$ & Padron, Kohinoor & Enzymes \\
\hline Caveolin & 4605,4604 & 9369,9370 & AgeI, NotI \\
Centromeres & 914,3978 & 9362,9376 & NheI, XhoI \\
Cytosolic & 4605,4604 & 9369,9370 & AgeI, NotI \\
EB3 & 2244,8650 & 9366,9367 & AgeI, NotI \\
ER & 916,9338 & 9364,9365 & SalI, NotI \\
Histone H2bn & 9337,8784 & 9372,9373 & NheI, BglII \\
Keratin & 5065,8650 & 9371,9367 & KpnI, NotI \\
Lifeact & 9340,9341 & 9374,9375 & BamHI, NotI \\
Map2 & 914,9336 & 9362,9363 & NheI, BglII \\
Mitochondria & 2244,8650 & 9366,9367 & AgeI, NotI \\
Nuclear pore complex & 9337,8784 & 9372,9373 & NheI, BglII \\
Peroxisomes & 2884,9336 & 9368,9363 & NheI, BglII \\
Tubulin & 914,9336 & 9362,9363 & NheI, BglII \\
Vimentin & 2244,8650 & 9366,9367 & AgeI, NotI \\
\hline
\end{tabular}

Actin For labeling of actin with Padron variants, the coding sequence of EGFP was replaced with the sequences coding for the fluorescent proteins in the lifeact-EGFP pcDNA 3.1(+) vector, which was a gift from Dr. Carola Gregor. A detailed plasmid map is shown in figure 5.1. Lifeact is a small peptide consisting of 17 amino acids originating from Saccharomyces cerevisiae that is capable of labeling F-actin [204]. Correct insertion was controlled with sequencing primer 7124 . 


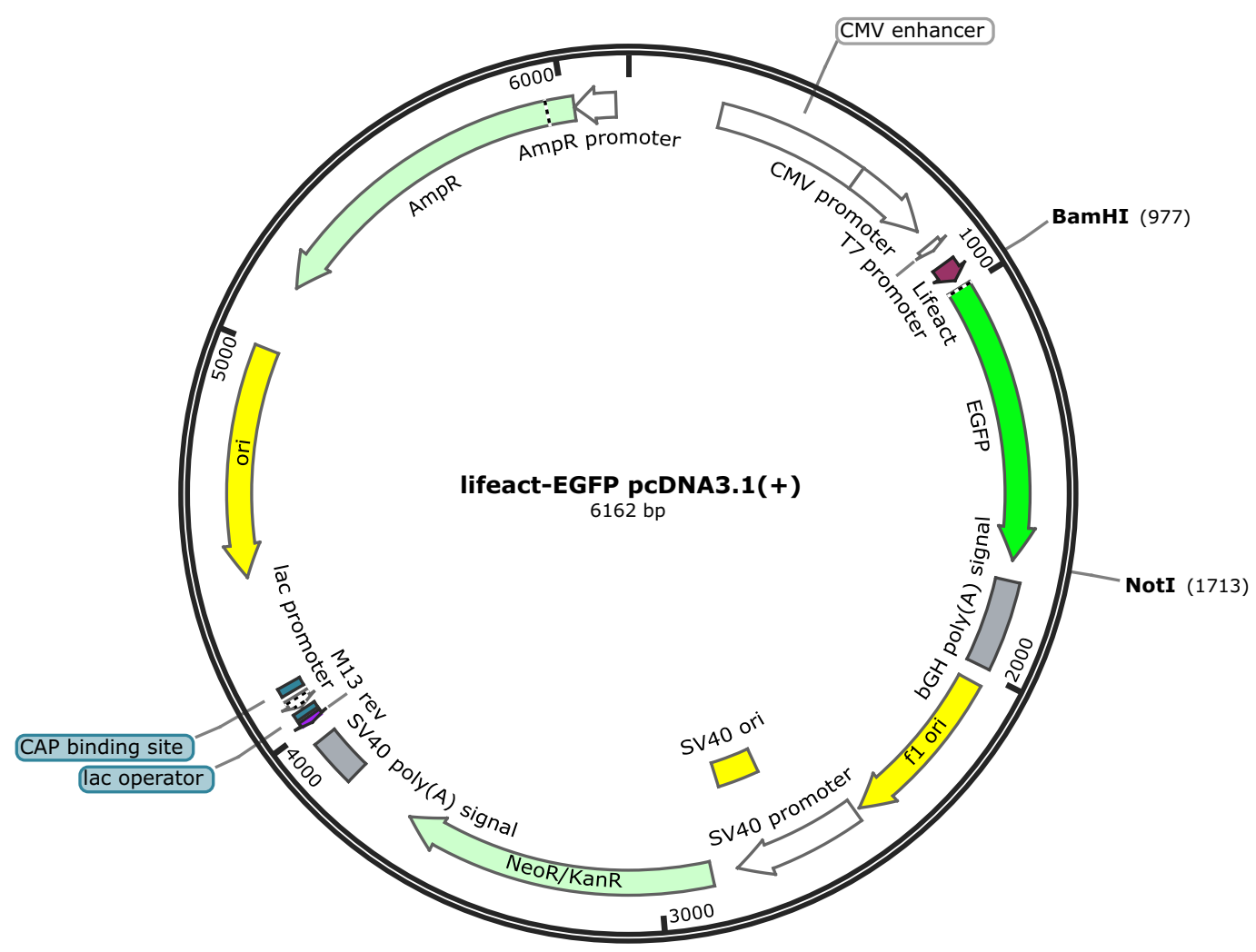

Figure 5.1: Lifeact EGFP pcDNA 3.1(+) plasmid map. The map was generated with SnapGene® 4.3.10 (GSL Biotech, Chicago, IL, USA) with the sequence provided by Dr. Carola Gregor. AmpR, ampicillin resistance; bGH, bovine growth hormone; CAP, catabolite activator protein; CMV, cytomegalovirus; KanR, kanamycin resistance; NeoR, neomycin resistance; ori, origin of replication; poly(A), polyadenylation; SV40, simian-virus 40.

Caveolin For expression in caveolae Padron variants were fused to caveolin. The coding sequences were inserted into caveolin1-mEGFP-N, which had previously been cloned in our group. This plasmid had been created by PCR amplification of the coding sequence for mEGFP with primers 1836 and 1837 and insertion into TagRFP-N (Evrogen, Moscow, Russia) with restriction enzymes AgeI and NotI resulting in mEGFP-N. The coding sequence for caveolin had then been PCR amplified from pDONR223-CAV1 [205] with primers 4571 and 4572 and had been inserted into mEGFP-N with restriction enzymes NheI and BamHI. Correct replacement of mEGFP with Padron coding sequences in caveolin1-mEGFP-N was controlled with sequencing primer 4667.

Centromeres For expression of fusion constructs with centromere protein C1 (CenpC1), Padron variants were inserted into a plasmid that had previously been cloned in our group. For this purpose, the coding sequence for the CENPC gene had first been amplified from pDONR223-CenpC [205] with primers 4552 and 4553 and had then been inserted into pEGFP-Tub (BD Biosciences Clontech, Franklin Lakes, NJ, USA) with restriction enzymes XhoI and BamHI, replacing the coding sequence for tubulin. Correct insertion of Padron variants was controlled with sequencing primer 928. 
Cytosol To express unfused Padron variants localized to the cytosol, coding sequences for the fluorescent proteins were inserted into TagRFP-N (Evrogen, Moscow, Russia), replacing TagRFP. Correct insertion was controlled with sequencing primer 2268.

Endoplasmic reticulum To localize Padron variants to the ER, coding sequences of Padron variants were inserted into $\mathrm{pEF} / m y c / \mathrm{ER}$ (Invitrogen Life Technologies, Carlsbad, CA, USA). Localization to the ER is facilitated by N-terminal fusion of an ER signal peptide and C-terminal addition of an ER retention signal. Correct insertion was controlled with sequencing primer 1016.

Histone For labeling of histones Padron variants were fused to histone H2bn. For this purpose, the desired protein was inserted into pEGFP-Hist1H2BN [175], effectively replacing EGFP. Correct insertion was controlled with sequencing primer 928.

Keratin To create fusion constructs of Padron variants and human cytokeratin18, the coding sequences for the desired proteins were inserted into pTagRFP-keratin (Evrogen, Moscow, Russia) replacing pTagRFP. Correct insertion was controlled with sequencing primer 4667 .

Microtubule ends To label microtubule filament ends, the coding sequence for human microtubule-associated factor EB3 had previously been PCR amplified from ptagRFP657-EB3 [206] and inserted into TagRFP-N (Evrogen, Moscow, Russia) with restriction enzymes SalI and BamHI in our group. TagRFP in this construct was replaced with the respective Padron variant. Correct insertion was controlled with sequencing primer 4667.

Microtubuli For indirect labeling of tubulin filaments, coding sequences of Padron variants were inserted into the plasmid rsFastLime-GFPend-Map2 that had previously been created in our group. To this end, rsFastLime-GFPend had been PCR amplified with primers 914 and 915 and inserted into the pEGFP-Tub plasmid (BD Biosciences Clontech, Franklin Lakes, NJ, USA) with restriction enzymes NheI and BglII. The tubulin coding sequence of the resulting plasmid had then been replaced with XhoI and BamHI by the gene coding for microtubuleassociated protein 2 (Map2), which had been PCR amplified from pDONR223-MAP2 [205]. Correct insertion of coding sequences were controlled with sequencing primer 928.

Mitochondria Mitochondrial localization of Padron variants was achieved by insertion of the coding sequence into pDsRed1-Mito (BD Biosciences Clontech, Franklin Lakes, NJ, USA). This plasmid N-terminally adds the human cytochrome c oxidase subunit VIII mitochondrial targeting sequence $[207,208]$ to the protein of interest, ensuring mitochondrial import. Correct insertion was controlled with sequencing primer 4667.

Nuclear pore complex For labeling of nuclear pore complexes Padron variants were fused to human nucleoporin $50 \mathrm{kDa}$ (Nup50). Coding sequences were inserted into 


\section{Methods}

mEmerald-Nup50-C-10, which was a gift from Michael Davidson (Addgene plasmid \# 54209), replacing mEmerald. Correct insertion was controlled with sequencing primer 2268.

Peroxisomes For peroxisomal localization of Padron variants mEGFP from pEGFP-PTS [175] was replaced with the desired RSFP coding sequence. This plasmid fuses a peroxisomal targeting sequence (PTS) to the C-terminal end of the inserted protein. Correct insertion was controlled with sequencing primer 928 .

Tubulin For expression of tubulin fusion constructs Padron variants were cloned into the pEGFP-Tub plasmid (BD Biosciences Clontech, Franklin Lakes, NJ, USA), replacing EGFP. Correct insertion was controlled with sequencing primer 928.

Vimentin For expression of vimentin fusion constructs Padron variants were inserted into pmKate2-vimentin vector (Evrogen, Moscow, Russia). Fluorescent proteins are C-terminally fused to vimentin. Correct insertion was controlled with sequencing primer 4667 .

\subsubsection{Cell line and growth conditions}

Expression of fusion constructs and microscopy were performed with transiently transfected HeLa cells. Cells were maintained in Dulbecco's Modified Eagle Medium (DMEM) with high glucose $(4.5 \mathrm{~g} / \mathrm{L})$, GlutaMAX ${ }^{\mathrm{TM}}$ and sodium pyruvate $(1 \mathrm{mM})$ (Thermo Fisher Scientific, Waltham, MA, USA) and $10 \%$ (v/v) fetal bovine serum (FBS) superior (Merck, Darmstadt, Germany). $100 \mathrm{U} / \mathrm{ml}$ penicillin and $100 \mathrm{\mu g} / \mathrm{ml}$ streptomycin were added to prevent bacterial contamination. Cells were proliferated at $37^{\circ} \mathrm{C}, 5 \% \mathrm{CO}_{2}$, and $90 \%$ humidity and regularly split before reaching confluency. For this purpose, medium was removed and cells were washed with phosphate buffered saline (PBS). Cells were covered with trypsin/EDTA (Merck, Darmstadt, Germany) and incubated at $37^{\circ} \mathrm{C}$ for several minutes. Cell detachment was stopped by addition of DMEM, and cells were transferred to a new flask.

\subsubsection{Transfection}

To transiently transfect HeLa cells with expression plasmids, cells were seeded in 6-well plates (Sarstedt, Nümbrecht, Germany) the day before transfection with two $18 \mathrm{~mm}$ glass coverslips No.1.5H (Paul Marienfeld, Lauda-Königshofen, Germany) placed in each well. For each transfection and well, $3 \mu \mathrm{g}$ plasmid were diluted in $400 \mu \mathrm{l}$ serum-free DMEM. $6 \mu \mathrm{l}$ TurboFect ${ }^{\mathrm{TM}}$ transfection reagent (Thermo Fisher Scientific, Waltham, MA, USA) were added and incubated for 15-20 min at room temperature. The mixture was added dropwise to the respective well and cells were incubated as described above until further use. 


\subsection{Mutagenesis}

\subsubsection{Cloning protocols}

Fluorescent proteins that were cloned into a new vector were PCR amplified from existing plasmids. Approximately $300 \mathrm{ng}$ plasmid were prepared in PCR buffer (see section 6.1) at a total volume of $50 \mathrm{\mu l}$ containing $0.2 \mathrm{mM}$ of each dNTP (Thermo Fisher Scientific, Waltham, MA, USA), $0.3 \mu \mathrm{M}$ of both forward and reverse primer, and $1 \mu \mathrm{l} P f u$ polymerase (biotechrabbit, Henningsdorf, Germany). PCR amplification (and all PCRs described below) was done either with the Biometra $\mathrm{T}_{O N E}$ or the Biometra $\mathrm{T}_{\text {personal }}$ PCR cycler (Analytik Jena, Jena, Germany) following the protocol listed in table 5.2 (annealing temperature and elongation duration were adjusted for different templates such as fusion constructs in mammalian expression vectors).

Table 5.2: PCR amplification PCR cycler protocol

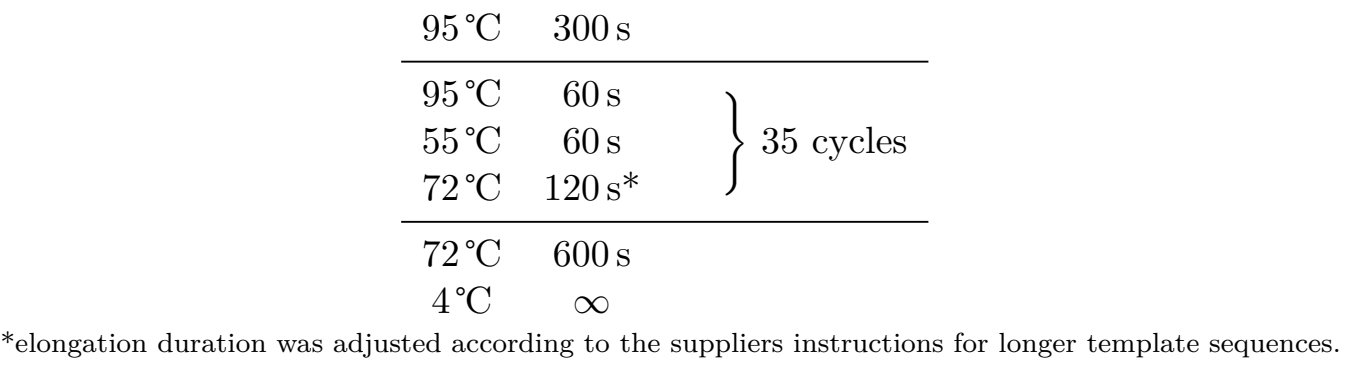

Backbone DNA was digested with restriction enzymes according to the product instructions in buffer supplied with the enzyme (all restriction enzymes: Thermo Fisher Scientific, Waltham, MA, USA). The restriction was incubated at $37^{\circ} \mathrm{C}$ for $2-3 \mathrm{~h} .1 \mu \mathrm{l} \mathrm{FastAP} \mathrm{(Thermo}$ Fisher Scientific, Waltham, MA, USA) was added for the last 30 minutes of incubation for DNA dephosphorylation to prevent empty vector ligation. Enzymes were inactivated by incubation at $80^{\circ} \mathrm{C}$ for 20 minutes. Restricted backbone and PCR amplification products were purified by agarose gel electrophoresis. To this end, RNase- and DNase-free agarose (BioBudget Technologies, Krefeld, Germany) was dissolved in 1x TAE-buffer at $1.2 \%(\mathrm{w} / \mathrm{v})$ in a microwave. Gels were casted and later run in a BlueMarine ${ }^{\mathrm{TM}} 100$ gel chamber (Serva Electrophoresis, Heidelberg, Germany). Restricted DNA samples were mixed with loading buffer and applied to the gel. Electrophoresis was run at $80 \mathrm{~V}$ with a $300 \mathrm{~V}$ power source (VWR, Radnor, PA, USA) for approximately $1 \mathrm{~h}$, and gels were subsequently stained with ethidium bromide. Desired DNA fragments were cut out after detection with UV light and isolated from the gel slice with either the QIAquick gel extraction kit (Qiagen, Hilden, Germany) or the NucleoSpin ${ }^{\circledR}$ Gel and PCR Clean-up kit (Macherey-Nagel, Düren, Germany) according to the product instructions. DNA was eluted in $30 \mu \mathrm{l}$ water. PCR products were digested according the protocol described above, purified with the QIAquick PCR Purification Kit (Qiagen, Hilden, Germany), and eluted in $30 \mu \mathrm{l}$ water. $2 \mu \mathrm{l}$ backbone and $6 \mu \mathrm{l}$ insert were used for subsequent ligation with $0.5 \mu \mathrm{l}$ T4 DNA ligase at a total volume of $20 \mu \mathrm{l}$ T4 DNA 


\section{Methods}

ligase buffer (Thermo Fisher Scientific, Waltham, MA, USA). Ligations were incubated over night at $4{ }^{\circ} \mathrm{C}$ and inactivated for 15 minutes at $65^{\circ} \mathrm{C}$. After dialysis for $45 \mathrm{~min}$ with $0.025 \mu \mathrm{m}$ MCE MF-Millipore ${ }^{\mathrm{TM}}$ membranes (Merck, Darmstadt, Germany), $5 \mu$ l ligation sample were used for transformation of electrocompetent E. coli.

\subsubsection{Site-directed mutagenesis}

For site-directed mutagenesis of Padron, approximately $200 \mathrm{ng}$ pBAD template construct were prepared in PCR buffer with $0.2 \mu \mathrm{M}$ of both forward and reverse primer, $0.2 \mathrm{mM}$ of each dNTP (Thermo Fisher Scientific, Waltham, MA, USA) and $1 \mu \mathrm{l}$ Pfu polymerase (biotechrabbit, Henningsdorf, Germany) at a total volume of $50 \mu$ l. Mutagenesis was then performed with the PCR protocol listed in table 5.3. Template DNA was digested with $1 \mu \mathrm{l}$ DpnI (Thermo Fisher Scientific, Waltham, MA, USA) at $37^{\circ} \mathrm{C}$ over night. Samples were dialyzed for $45 \mathrm{~min}$ with $0.025 \mu \mathrm{m}$ MCE MF-Millipore ${ }^{\mathrm{TM}}$ membranes (Merck, Darmstadt, Germany), and $5 \mu \mathrm{l}$ were used for transformation of electrocompetent E. coli.

Table 5.3: Site-directed mutagenesis PCR cycler protocol

\begin{tabular}{|c|c|c|}
\hline $95^{\circ} \mathrm{C}$ & $30 \mathrm{~s}$ & \\
\hline $95^{\circ} \mathrm{C}$ & $60 \mathrm{~s}$ & \multirow{3}{*}{16 cycles } \\
\hline $55^{\circ} \mathrm{C}$ & $60 \mathrm{~s}$ & \\
\hline $72^{\circ} \mathrm{C}$ & $600 \mathrm{~s}^{*}$ & \\
\hline $72^{\circ} \mathrm{C}$ & $600 \mathrm{~s}$ & \\
\hline $4^{\circ} \mathrm{C}$ & $\infty$ & \\
\hline
\end{tabular}

\subsubsection{Multiple-site mutagenesis}

To introduce multiple mutations into Padron in the pBAD vector, multiple-site mutagenesis (MSM) was performed [209]. Approximately $500 \mathrm{ng}$ pBAD template construct were prepared in 0.5x PCR buffer and 0.5x Taq ligase buffer (New England Biolabs, Ipswich, MA, USA) with

Table 5.4: Multiple-site mutagenesis PCR cycler protocol

MSM PCR profile 1

\begin{tabular}{|c|c|c|}
\hline $95^{\circ} \mathrm{C}$ & $120 \mathrm{~s}$ & \\
\hline $95^{\circ} \mathrm{C}$ & $30 \mathrm{~s}$ & \multirow{3}{*}{18 cycles } \\
\hline $55^{\circ} \mathrm{C}$ & $30 \mathrm{~s}$ & \\
\hline $65^{\circ} \mathrm{C}$ & $600 \mathrm{~s}$ & \\
\hline $75^{\circ} \mathrm{C}$ & $420 \mathrm{~s}$ & \\
\hline
\end{tabular}

MSM PCR profile 2

\begin{tabular}{|c|c|c|}
\hline $95^{\circ} \mathrm{C}$ & $30 \mathrm{~s}$ & \\
\hline $95^{\circ} \mathrm{C}$ & $30 \mathrm{~s}$ & \\
\hline $55^{\circ} \mathrm{C}$ & $60 \mathrm{~s}$ & 2 cycles \\
\hline $70^{\circ} \mathrm{C}$ & $600 \mathrm{~s}$ & \\
\hline
\end{tabular}


5'-phosphorylated forward mutagenesis primers $(0.28 \mu \mathrm{M})$ and $0.2 \mathrm{mM}$ of each dNTP (Thermo Fisher Scientific, Waltham, MA, USA). $1 \mu \mathrm{l}$ Pfu polymerase (biotechrabbit, Henningsdorf, Germany) and 0.5 $\mathrm{\mu l}$ Taq DNA ligase (New England Biolabs, Ipswich, MA, USA) were added, total volume was $50 \mathrm{\mu l}$. MSM PCR profile 1 (table 5.4) was performed. After completion, $1 \mathrm{\mu l}$ DpnI (Thermo Fisher Scientific, Waltham, MA, USA) and $1.5 \mu$ primer 1640 (10 $\mu \mathrm{M}$ stock) were added and MSM PCR profile 2 (table 5.4) was performed. Samples were dialyzed for 45 min with 0.025 $\mathrm{mm}$ MCE MF-Millipore ${ }^{\mathrm{TM}}$ membranes (Merck, Darmstadt, Germany). $5 \mathrm{\mu l}$ of the MSM product were used for transformation of electrocompetent cells.

\subsubsection{Error-prone mutagenesis}

For generation of random mutations in fluorescent proteins, error-prone mutagenesis was performed. Approximately $1 \mu g$ template DNA in a final volume of $100 \mu l$ in error-prone PCR buffer were mixed with forward and reverse primers at $1 \mu \mathrm{M}$ concentration, $10 \mu \mathrm{l}$ error-prone dNTP-mix and $2 \mu$ l GoTaq ${ }^{\circledR}$ DNA polymerase (Promega, Madison, WI, USA). $\mathrm{MnCl}_{2}$ was added at a final concentration of $0.2-0.3 \mathrm{mM}$ depending on the desired number of mutations. Prior to the PCR amplification this mixture was split into 4 vials, and PCR was performed with the protocol listed in table 5.5. Split samples were combined and PCR amplified DNA fragments along with the desired vector for expression were purified, restricted, and ligated as described in section 5.2.1. Restriction enzymes used for fluorescent protein expression vectors are given in section 5.1.1.1. $5 \mu \mathrm{l}$ of the ligation sample were used for transformation of electrocompetent cells.

Table 5.5: Error-prone mutagenesis PCR cycler protocol

\begin{tabular}{|c|c|c|}
\hline $95^{\circ} \mathrm{C}$ & $30 \mathrm{~s}$ & \multirow{3}{*}{30 cycles } \\
\hline $55^{\circ} \mathrm{C}$ & $45 \mathrm{~s}$ & \\
\hline $72^{\circ} \mathrm{C}$ & $60 \mathrm{~s}$ & \\
\hline $72^{\circ} \mathrm{C}$ & $120 \mathrm{~s}$ & \\
\hline $4^{\circ} \mathrm{C}$ & $\infty$ & \\
\hline
\end{tabular}

\subsubsection{Plasmid isolation from bacterial cultures}

Plasmid DNA from bacteria harboring newly cloned constructs or mutagenized vectors was isolated from overnight LB liquid cultures inoculated with a single bacterial colony. Plasmids were either isolated in low amounts with the QIAprep Spin Miniprep Kit (Qiagen, Hilden, Germany) from culture volumes of $5-10 \mathrm{ml}$ or in larger amounts from $50 \mathrm{ml}$ cultures with the Plasmid Plus Midi Kit (Qiagen, Hilden, Germany). DNA concentration was measured with a NanoDrop 1000 spectrophotometer (peqlab, Erlangen, Germany). Plasmids were stored at $4^{\circ} \mathrm{C}$ for short periods or frozen at $-20^{\circ} \mathrm{C}$ for long term storage. 


\section{Methods}

\subsubsection{DNA sequencing}

All DNA sequencing was performed externally by Microsynth Seqlab (Göttingen, Germany). For sample preparation, plasmid DNA was prediluted in sterile water to approximately $80 \mathrm{ng} / \mu \mathrm{l}$, and the sequencing primer was added at a final concentration of $2.5 \mu \mathrm{M}$. All sequencing primers used in this thesis are listed in table 6.1 in section 6.3.

\subsection{Screening}

Mutagenized expression plasmids of Padron were transformed into electrocompetent E. coli which were used for inoculation of LB-plates supplied with antibiotics. The plates were incubated at either $30^{\circ} \mathrm{C}$ or $37^{\circ} \mathrm{C}$, depending on the variant and the parameter to be analyzed in the following screening. Incubation times varied as well based on the ability of the respective template variant to mature. Screenings were performed at room temperature, and plates were equilibrated beforehand to eliminate temperature dependencies of parameters analyzed.

\subsubsection{Automated bacterial screening}

All screenings were performed with a customized DM5500 B microscope (Leica Microsystems, Wetzlar, Germany). The microscope had previously been modified in our group to be able to automatically screen bacterial colonies on LB-agar plates and to measure time-resolved fluorescence in bacterial colonies over a large spectral range from ultraviolet to infrared light. The following section will only describe parts and modifications relevant to this thesis.

\subsubsection{Technical specifications of the screening setup}

Optics and other parts The microscope was equipped with a SCAN 100x100 stage (Märzhäuser Wetzlar, Wetzlar, Germany) controlled by the CTR6500 stage controller and an N PLAN L 20x/0.40 objective (both Leica Microsystems, Wetzlar, Germany). The filter cube used for all screenings contained a $495 \mathrm{~nm}$ DCXR beamsplitter and a $488 \mathrm{~nm}$ long pass detection filter. The microscope was positioned on a stabilized optical table along with MLD ${ }^{\text {TM }} 405 \mathrm{~nm}$ and $488 \mathrm{~nm}$ lasers (both Cobolt, Solna, Sweden). Both lasers were adjusted to a combined beam path with a dichroic mirror reflecting light between 375 and $405 \mathrm{~nm}$. Lasers were directed into the opened back of the microscope stand top. Light was focused on bacterial colonies, emitted fluorescence was collected through the open camera port of the microscope and focused through a pinhole on a H10723 series photomultiplier tube (PMT) photosensor (Hamamatsu, Hamamatsu, Japan). Laser powers were controlled with acoustooptic modulators (488 nm: MTS 110/A3 VIS, AA OPTO-ELECTRONIC, Orsay, France; 405 nm: 308-125, Crystal Technology, Palo Alto, CA, USA).

Software and laser control Control of microscope and lasers was achieved with extensive software written previously in our group with LabVIEW (National Instruments, Austin, TX, USA). In principle, the software allowed precise and automated movement of the microscope 
stage for screening bacterial colonies on LB-agar as well as precisely timed laser control. At every position, an autofocus was performed by movement of the stage along the optical axis to ensure maximal signal intensity (colony sampling and autofocus were improved in the course of this thesis, details are described in section 3.1). After focusing, a predefined laser sequence was run to induce photoswitching of fluorescent proteins. Measurements were subdivided into so called blocks: for each block, the user could define parameters such as laser intensity, duration, and timing prior to a screening. The number and sequence of block repetitions run at each position was adjustable as well. A typical measurement for screening of Padron consisted of an initial block with the $405 \mathrm{~nm}$ laser activated to switch proteins into the off-state, followed by a second block with the $488 \mathrm{~nm}$ laser activated to return the protein to the on-state. These two blocks were repeated 250 times to estimate switching fatigue. This allowed for unsupervised measurement of residual off-state fluorescence, fluorescence intensity, switching speed and switching fatigue of approximately 1000 colonies on a $12 \times 12 \mathrm{~cm}$ agar plate. Data visualization and analysis was done in the LabVIEW data analysis program written previously in the group.

In the course of the thesis, the timing precision of the screening setup underwent substantial changes with the implementation of a field programmable gate array (FPGA) upgrade implemented by Dr. Martin Andresen. Before the upgrade, laser control was done solely based on LabVIEW code run on a computer, which caused a delay of a few milliseconds between the execution of the code and actual adjustment of laser power. As a consequence, after data recording for one block (e.g. switching the protein to the off-state) was stopped, the laser was deactivated a few milliseconds later, effectively increasing the light dose applied for off-switching. This delay varied based on the CPU usage and was in the range between 3-12 ms. All Padron variants up to and including Padron 1.9 were screened under these conditions. Following screenings and, most importantly, all characterization data shown in this thesis were screened after implementation of the FPGA upgrade. The FPGA card allowed for microsecond precision with respect to laser control. The measurement blocks were written to a field programmable gate array prior to the start of a measurement and were executed solely based on the hardware timing of the FPGA card independent from the CPU usage of the controlling computer. However, with the current version of the software, there was a minor drawback of the system. Due to the limited size of the FPGA memory, the complete execution code for very long measurements in the minute range could not be written into this memory. In this case, the measurements had to be interrupted to reprogram the FPGA card. This did not cause delayed laser activation or inactivation but introduced short breaks in the lower millisecond range during reprogramming. For the experiments described in this thesis, this drawback only had an effect on photobleaching measurements and analysis of switching fatigue with 1000 switching cycles.

Power calculation Laser power applied in all experiments was measured behind the objective with the LabMax-TO laser power meter equipped with an LM-2 VIS sensor (both Coherent, Santa Clara, CA, USA). To calculate laser intensities, lasers were focused on a 


\section{Methods}

mirror surface. The reflected light entered the detection path by means of a filter cube equipped only with a 50/50 beam splitter. Light was redirected with a removable mirror to a ICX098BQ CCD image sensor (Sony, Minato, Japan) of a SPC900NC webcam (Philips, Amsterdam, Netherlands) where lens and housing had been removed. Pixel sizes were calibrated with the image of a $10 \mu \mathrm{m}$ scale recorded as a snapshot of a video feed. Focal spots were recorded accordingly with $640 \times 480$ pixel resolution while care was taken to prevent over or under exposure of the sensor. Intensity line profiles were measured in 4 equally spaced orientations (line width: 1 pixel) through the center of the focal spot with the Fiji distribution of ImageJ (v1.52p, [166, 167]) and individually fitted with a Gaussian nonlinear curve fit in OriginPro 2018b (OriginLab Corporation, Northampton, MA, USA). FWHM values were averaged and the focal spot area was calculated as circle with the FWHM as diameter.

\subsubsection{Improved autofocus evaluation}

During the course of this thesis, the autofocus of the automated screening microscope was improved, which is described in detail in section 3.1.2. Precision of the new autofocus system was evaluated in comparison with TOP10 E. coli expressing mNeonGreen [168]. The coding sequence for mNeonGreen had previously been cloned into the $\mathrm{pBAD}$ vector in the lab after it had been synthesized (Eurofins genomics, Luxembourg, Belgium). Colonies were localized with the improved sampling (cf. sec. 3.1.1) and measured with a function specifically written for this purpose. This program sequentially performed the old as well as the new autofocus. The old autofocus was performed with 80 steps over a range of $2.5 \mathrm{~mm}$, whereas the new one was measured over a range of $5 \mathrm{~mm}$ within $1.3 \mathrm{~s}$. Fluorescence was then probed for every colony at the same coordinates at the focal positions from both methods. Focal differences and peak intensities at the respective focal positions were analyzed.

\subsubsection{Screening strategy}

Padron was mutagenized in either the pQE-31 or the pBAD vector (see section 5.1.1.1 for details) in multiple rounds with the protocols described in section 5.2. After every round of mutagenesis, E. coli were transformed and plated on 12x12 cm LB-agar plates and incubated at $37^{\circ} \mathrm{C}$ over night or at $30^{\circ} \mathrm{C}$ for up to 2 days. Temperature and incubation times were adjusted depending of the capability of the respective template variant to mature at $37^{\circ} \mathrm{C}$. After incubation and adjustment to room temperature, one or more plates were screened for improved protein variants. Screening data was analyzed with the LabVIEW analysis software to reveal improved switching parameters such as lower residual off-state fluorescence or enhanced switching fatigue of mutated Padron variants. Promising clones were picked and used for inoculation of $5 \mathrm{ml}$ over night cultures. The next day, plasmid DNA was isolated and sequenced as described in sections 5.2.5 and 5.2.6. Plasmids harboring new mutations were retransformed into electrocompetent $E$. coli which were plated and incubated as described above. These clones were remeasured in multiple colonies and compared to the template protein, improved variants were subjected to further mutagenesis. 
Padron 1.9 was the result of a screening in which transformed E. coli were preselected by fluorescence activated cell sorting (FACS) with a customized BD Influx ${ }^{\mathrm{TM}}$ flow cytometer (BD Biosciences, Franklin Lake, NJ, USA). For this purpose, TOP10 cells from an overnight culture transformed with plasmids from an error-prone mutagenesis were used for inoculation of $5 \mathrm{ml} \mathrm{LB}$-medium with $0.02 \%$ (w/v) arabinose to induce expression of fluorescent proteins. Bacterial cells were washed with PBS and sorted $3 \mathrm{~h}$ and 30 min later in the logarithmic growth phase. The brightest clones were sorted to a LB-plate and analyzed with the automated screening microscope after overnight growth.

Padron 2.1 resulted from a directed evolution approach. Padron 2.0 was mutagenized in 6 consecutive error-prone mutageneses. In each round, bacterial colonies were screened for improved variants, and approximately 10 of the most promising variants were picked and used for inoculation of $5 \mathrm{ml}$ overnight cultures. Plasmids were isolated but retransformation as well as comparison to the template were omitted. Instead, all vectors from the last round were mixed at equal concentrations and used as template for the next round. After 6 rounds of mutagenesis, selected clones were sequenced and compared to Padron 2.0, yielding Padron 2.1 as the most promising one.

Over the course of all mutageneses the DNA sequence coding for Padron variants accumulated several silent mutations. In order to restore sequence identity to the original Padron at these positions, multiple-site mutagenesis was applied to plasmids encoding Padron 1.9, 2.0, and

2.1. Primers used are listed in table 6.2.

\subsubsection{Fluorescent protein characterization in bacterial colonies}

Comparative measurements of all Padron variants The data displayed section 3.3 were measured on one day. pBAD vector transformed TOP10 E. coli were grown at $37^{\circ} \mathrm{C}$ for $24 \mathrm{~h}$ and for $50 \mathrm{~h}$ at $30^{\circ} \mathrm{C}$. Plates were adjusted to room temperature for at least $2 \mathrm{~h}$ prior to measurements. For each protein and both growth conditions, 10 colonies were measured. All proteins were measured with equal laser intensities $\left(405 \mathrm{~nm}\right.$ at $3.6 \mathrm{~kW} / \mathrm{cm}^{2}, 488 \mathrm{~nm}$ at $17.2 \mathrm{~kW} / \mathrm{cm}^{2}$ ) and illumination sequences. If the PMT was saturated under these conditions, neutral grey density filters were used, and the actual fluorescence intensity was calculated based on the attenuation of the filters. Fluorescence intensity, switching half-time, residual off-state fluorescence, relative on-state fluorescence after 250 cycles, and maximum/endfluorescence intensity were calculated from data measured with scheme A, residual off-state fluorescence intensity with simultaneous $488 \mathrm{~nm}$ illumination data is based on scheme B (table 5.6). Sampling rate was $10 \mu$ s. Data was analyzed with the LabVIEW analysis software ignoring the first cycle to exclude influence of the equilibrium state on parameters such as residual off-state fluorescence intensity.

For characterization measurements at the automated screening microscope described below, Padron variants were expressed in TOP10 E. coli and incubated for $24 \mathrm{~h}$ at $37^{\circ} \mathrm{C}$. Growth conditions were different only for the original Padron and pa69, which had to be expressed 


\section{Methods}

for $48 \mathrm{~h}$ at $30^{\circ} \mathrm{C}$ for full expression and maturation. After incubation, all bacterial plates were kept at room temperature over night to ensure full maturation of fluorescent proteins.

Table 5.6: Padron comparison illumination sequences

\begin{tabular}{lccc} 
Scheme & Block 1 & Block 2 & Cycles \\
\hline \multirow{2}{*}{ A } & $6.5 \mathrm{~ms}$ & $10.0 \mathrm{~ms}$ & \multirow{2}{*}{250} \\
& $405 \mathrm{~nm}$ & $488 \mathrm{~nm}$ & \\
\hline \multirow{2}{*}{ B } & $6.5 \mathrm{~ms}$ & $10.0 \mathrm{~ms}$ & \multirow{2}{*}{150} \\
& $405 \& 488 \mathrm{~nm}$ & $488 \mathrm{~nm}$ & \\
\hline
\end{tabular}

Residual off-state fluorescence intensity in bacterial colonies To determine the lowest achievable ensemble switching background in bacterial colonies, $405 \mathrm{~nm}$ off-switching duration was modulated at the automated screening microscope. Residual off-state fluorescence intensity was then calculated from activation curves under $488 \mathrm{~nm}$ illumination at $20.2 \pm 0.9 \mathrm{~kW} / \mathrm{cm}^{2}$ with the LabView analysis software. For each condition 5 colonies were measured with 10 cycles of which the first cycle was ignored to exclude influence of the equilibrium state. $405 \mathrm{~nm}$ modulation was measured at 5 different intensities and measurements were independently repeated 3 times (Padron: 2) on different days. Residual off-state values for different $405 \mathrm{~nm}$ doses were averaged for different colonies. Repetitions were then averaged and fitted in OriginPro 2018b (OriginLab Corporation, Northampton, MA, USA) with the ExpDec2 nonlinear curve fit. Switching background was determined by averaging the last 12 data points of the repetition fits if residual off-state fluorescence declined continuously and was stationary for these data points.

Because Kohinoor, Padron, and Padron 1.9/2.0/2.1 showed strongly differing responsiveness to $405 \mathrm{~nm}$ illumination, the modulation had to be adapted; the exact illumination parameters are listed in table 5.7. To estimate the influence of high $405 \mathrm{~nm}$ doses applied to Kohinoor samples, Padron 2.0 was measured at 3 different powers in a single repetition with Kohinoor settings.

Table 5.7: $405 \mathrm{~nm}$ modulation illumination parameters

\section{$405 \mathrm{~nm}$ modulation}

\begin{tabular}{lccccc} 
Protein & shortest & longest & step & $\mathbf{4 8 8} \mathbf{~ n m}$ & sampling rate \\
\hline Padron & $10.0 \mathrm{~ms}$ & $300 \mathrm{~ms}$ & $10.0 \mathrm{~ms}$ & $80 \mathrm{~ms}$ & $50 \mu \mathrm{s}$ \\
Padron $1.9 / 2.0 / 2.1$ & $0.5 \mathrm{~ms}$ & $15 \mathrm{~ms}$ & $0.5 \mathrm{~ms}$ & $10 \mathrm{~ms}$ & $10 \mu \mathrm{s}$ \\
Kohinoor & $20.0 \mathrm{~ms}$ & $600 \mathrm{~ms}$ & $20.0 \mathrm{~ms}$ & $50 \mathrm{~ms}$ & $50 \mu \mathrm{s}$ \\
\hline
\end{tabular}


$488 \mathrm{~nm}$ switching kinetics To compare kinetics for switching to the on-state with $488 \mathrm{~nm}$ light and to analyze kinetic responsiveness, $488 \mathrm{~nm}$ intensity was modulated. Proteins were switched to $5 \%$ residual fluorescence of the on-state (Kohinoor: $10 \%$ ) with $405 \mathrm{~nm}$ at $3.6 \mathrm{~kW} / \mathrm{cm}^{2}$ and switched back to the on-state with varying $488 \mathrm{~nm}$ intensities. For every intensity, 10 colonies were measured, and the first cycle was ignored to exclude influence of the ground-state. Measurements were repeated 3 times for every protein on different days. Duration of $488 \mathrm{~nm}$ illumination was kept excessive to ensure full switching to the on-state. Data of each repetition was analyzed in OriginPro 2018b (OriginLab Corporation, Northampton, MA, USA). Single measurements of each repetition were smoothed, divided by the maximum value and averaged. Maxima indices of single measurements were averaged and data recorded beyond this value were discarded from the averaged curves to obtain full switching curves without fluorescence decay of the on-state due to photobleaching during excessive $488 \mathrm{~nm}$ illumination. Figures of full curves depict averaged repetitions.

Switching half-time from off- to on-state and switching to $95 \%$ of the full on-state was done after fitting averaged curves of each repetition with the ExpGro2 nonlinear curve fit. The first data point above $95 \%$ of the full on-state was determined from the fitted curves and repetitions were averaged. Switching half-time was determined from the fitted data accordingly. Laser intensity dependency of switching times was then fitted with the ExpDec2 fit.

Switching fatigue Bleaching in response to repeated switching was analyzed by performing 1000 consecutive switching cycles in bacterial colonies. Due to the large size of datasets, measurements could not be performed in a single FPGA-based sequence so that a short delay in the low millisecond range had to be introduced between switching cycles (see section 5.3.1 for details). Illumination times were adjusted for every protein to be representative of imaging applications. For every measurement, samples were switched to $5 \%$ residual fluorescence (Kohinoor: $10 \%$ ) with $3.6 \mathrm{~kW} / \mathrm{cm}^{2}$ as determined by $405 \mathrm{~nm}$ modulation followed by activation to $95 \%$ of the saturated on-state fluorescence intensity. 10 colonies were measured for every protein at two different $488 \mathrm{~nm}$ intensities $\left(2.6\right.$ and $\left.30.1 \mathrm{~kW} / \mathrm{cm}^{2}\right)$ and measurements were repeated 3 times on different days. To estimate influence of photobleaching due to differing light doses, Padron 2.0 was measured with Kohinoor settings with 2 repetitions, and Padron and Kohinoor were measured with Padron 2.0 settings with 1 or 2 repetitions, respectively. Detailed light doses are listed in table 5.8.

Table 5.8: Switching fatigue illumination times $405 \mathrm{~nm} \quad 488 \mathrm{~nm}$ illumination

\begin{tabular}{lccc} 
Protein & $\mathbf{3 . 6} \mathbf{~} \mathbf{W} / \mathbf{c m}^{2}$ & $\mathbf{2 . 6} \mathbf{~} \mathbf{W} / \mathbf{c m}^{2}$ & $\mathbf{3 0 . 1} \mathbf{~} \mathbf{W} / \mathbf{c m}^{2}$ \\
\hline Padron & $60.8 \mathrm{~ms}$ & $207.0 \mathrm{~ms}$ & $21.0 \mathrm{~ms}$ \\
Padron 1.9 & $5.3 \mathrm{~ms}$ & $86.2 \mathrm{~ms}$ & $5.8 \mathrm{~ms}$ \\
Padron 2.0 & $5.4 \mathrm{~ms}$ & $73.1 \mathrm{~ms}$ & $5.9 \mathrm{~ms}$ \\
Padron 2.1 & $2.0 \mathrm{~ms}$ & $86.5 \mathrm{~ms}$ & $7.8 \mathrm{~ms}$ \\
Kohinoor & $164.5 \mathrm{~ms}$ & $124.9 \mathrm{~ms}$ & $9.5 \mathrm{~ms}$ \\
\hline
\end{tabular}




\section{Methods}

Start and end data points of all activation curves were extracted from the raw data with the LabVIEW analysis software and were further analyzed with OriginPro 2018b (OriginLab Corporation, Northampton, MA, USA). Single measurements were first divided by the maximal value and subsequently averaged.

Photobleaching in bacterial colonies For each protein, fluorescence was recorded in 5-10 unique colonies with 3-4 independent repetitions on different days. Photobleaching was recorded for $10 \mathrm{~min}$ with $488 \mathrm{~nm}$ excitation at $2.3 \pm 0.3 \mathrm{~kW} / \mathrm{cm}^{2}$ and for $3 \mathrm{~min}$ with $488 \mathrm{~nm}$ excitation at $30.8 \pm 1.5 \mathrm{~kW} / \mathrm{cm}^{2}$. Data was normalized and averaged with 1000 data points of the full $\mathrm{x}$ range in OriginPro 2018b (OriginLab Corporation, Northampton, MA, USA). Time to bleach to $50 \%$ of the maximal intensity was determined from averaged repetitions as the first data point below $50 \%$. After averaging, sampling rate was $0.62 \mathrm{~s}$ for $10 \mathrm{~min}$ and $0.18 \mathrm{~s}$ for 3 min datasets.

Residual fluorescence in the off-state with simultaneous $488 \mathbf{~ n m}$ illumination To estimate efficiency of $405 \mathrm{~nm}$ switching to the off-state under constant $488 \mathrm{~nm}$ illumination, bacterial colonies were switched with 2 different $405 \mathrm{~nm}$ intensities $\left(2.3\right.$ and $\left.33.1 \mathrm{~kW} / \mathrm{cm}^{2}\right)$ in combination with 2 different $488 \mathrm{~nm}$ intensities $\left(1.1\right.$ and $\left.15.0 \mathrm{~kW} / \mathrm{cm}^{2}\right)$. Return to the on-state was in all combinations of parameters facilitated with $488 \mathrm{~nm}$ at $15.0 \mathrm{~kW} / \mathrm{cm}^{2}$. Illumination times for switching to the off-state were the maximal durations used for $405 \mathrm{~nm}$ modulation. Switching to the on-state was determined empirically based on test measurements, detailed parameters are listed in table 5.9. For every combination of intensities 5 cycles were measured in 10 colonies with 3 repetitions on different days. Residual fluorescence after switching to the off-state was determined with the LabVIEW analysis software for the second cycle to eliminate influence of the equilibrium state. Values were averaged.

Table 5.9: Residual fluorescence in the off-state with simultaneous $488 \mathrm{~nm}$ irradiation illumination times

\begin{tabular}{lcc} 
Protein & $\mathbf{4 0 5 + 4 8 8} \mathbf{~ n m}$ illumination & $\mathbf{4 8 8} \mathbf{~ n m}$ illumination \\
\hline Padron & $300 \mathrm{~ms}$ & $80 \mathrm{~ms}$ \\
Padron 1.9 & $15 \mathrm{~ms}$ & $25 \mathrm{~ms}$ \\
Padron 2.0 & $15 \mathrm{~ms}$ & $25 \mathrm{~ms}$ \\
Padron 2.1 & $15 \mathrm{~ms}$ & $35 \mathrm{~ms}$ \\
Kohinoor & $600 \mathrm{~ms}$ & $70 \mathrm{~ms}$ \\
\hline
\end{tabular}

\subsection{Protein purification}

For spectral measurements, gel electrophoresis, size exclusion chromatography, and in vitro switching, Padron, Kohinoor, and Padron 1.9/2.0/2.1 were purified using the following protocol. DsRed, dTomato and mEGFP were purified for comparison. 


\subsubsection{Protein expression}

pBAD plasmids coding for the proteins mentioned above were transformed into electrocompetent TOP10 E. coli. Cells were plated on LB-plates containing $0.02 \%(\mathrm{w} / \mathrm{v})$ arabinose, 2 plates were inoculated for each protein. Cultures were incubated at $37^{\circ} \mathrm{C}$ for $24 \mathrm{~h}$ (Kohinoor, Padron 1.9, 2.0 and 2.1) or at $30^{\circ} \mathrm{C}$ for $48 \mathrm{~h}$ (Padron). Plates were then kept at room temperature for another 16-24 h to ensure full maturation of expressed Padron variants.

\subsubsection{Cell lysis}

Cells were collected with a cell scraper in $1.8 \mathrm{ml}$ binding buffer (buffer concentrations are given in section 6.1), transferred to a $2 \mathrm{ml}$ vial, and resuspended. $40 \mathrm{\mu l}$ of a $50 \mathrm{mg} / \mathrm{ml}$ lysozyme stock solution (Serva electrophoresis, Heidelberg, Germany) were added to each vial, and cell suspensions were incubated for approximately $4 \mathrm{~h}$ on ice. $80 \mu \mathrm{l} \mathrm{cOmplete}{ }^{\mathrm{TM}}$ protease inhibitor cocktail (Roche, Basel, Switzerland) were added to each vial, and samples were then shock frozen in liquid nitrogen and thawed in lukewarm water 5 times. $0.5 \mu \mathrm{l}$ benzonase (Thermo Fisher Scientific, Waltham, MA, USA) were added to each vial, and samples were centrifuged at 21,000 rcf in a Heraeus Fresco 21 micro centrifuge (Thermo Fisher Scientific, Waltham, MA, USA) for $3-6 \mathrm{~h}$ at $4^{\circ} \mathrm{C}$. The supernatant was removed and stored in the fridge over night, precipitates were discarded.

\subsubsection{Ni NTA spin column protein isolation}

Proteins were purified from the crude lysate with the His SpinTrap kit (GE Healthcare, Chicago, IL, USA) following a modified protocol at room temperature. His SpinTrap columns were prepared with an initial washing step with $600 \mathrm{\mu l}$ binding buffer, which was removed and discarded by centrifugation at $100 \mathrm{rcf}$ for $1 \mathrm{~min}$ (all following centrifugation steps were employed under the same conditions). For each protein to be isolated three columns were used. $600 \mathrm{\mu l}$ crude lysate were added to each column and columns were rotated and incubated for $30 \mathrm{~min}$ in the dark. Lysate and unbound protein was removed by centrifugation, and this step was repeated if more lysate was available. All columns were then washed twice with $600 \mu \mathrm{l}$ binding buffer and twice with $200 \mu \mathrm{l}$ washing buffer. Proteins were then eluted in 3 consecutive steps. For each step $200 \mu \mathrm{l}$ elution buffer were added and eluted into a new microcentrifuge vial after 3 min incubation.

Buffer change After elution, samples containing the same protein were united and concentrated with Vivaspin ${ }^{\circledR} 500$ centrifugal concentrators with a molecular weight cut-off of $10,000 \mathrm{kDa}$ (Sartorius, Göttingen, Germany) to a volume of approximately $300 \mu \mathrm{l}$. The elution buffer was exchanged with standard protein buffer $(100 \mathrm{mM}$ Tris, $150 \mathrm{mM}$ sodium chloride, $\mathrm{pH} 7.5$ ) by use of NAP-5 columns (GE Healthcare, Chicago, IL, USA). The columns were equilibrated with $2.5 \mathrm{ml}$ protein buffer 3 times after which protein samples were added. Protein samples were collected after elution with standard protein buffer. For size exclu- 


\section{Methods}

sion chromatography analysis, protein solutions were additionally filtered with Vivaspin® 500 centrifugal concentrators with a pore size of $0.2 \mu \mathrm{m}$ (Sartorius, Göttingen, Germany).

\subsubsection{Protein concentration and purity}

Protein concentrations were measured with the Bio-Rad protein assay (Bio-Rad, Hercules, CA, USA) following the suppliers instructions. The assay is based on the Bradford method [210]. Albumin from bovine serum fraction V (Sigma-Aldrich, St. Louis, MO, USA) was used as protein standard with concentrations up to $10 \mathrm{mg} / \mathrm{ml}$. Protein standard $\mathrm{A}_{600 \mathrm{~nm}}$ values were modeled after equation 5.1, and sample concentrations were calculated accordingly.

$$
A_{600 \mathrm{~nm}}=a x^{2}+b x+c
$$

where $x$ is the protein concentration in $\mathrm{mg} / \mathrm{ml}$.

\subsubsection{Polyacrylamide gel electrophoresis}

To estimate purity and dimerization tendency of isolated proteins, denaturing and seminative polyacrylamide gel electrophoreses (PAGE) were performed. PAGE gels were casted and run with the Mini-PROTEAN Tetra handcast system (BioRad, Hercules, CA, USA) and a $300 \mathrm{~V}$ power source (VWR, Radnor, PA, USA).

\subsubsection{Denaturing PAGE}

Denaturing PAGE was performed with sodium dodecyl sulfate (SDS) added to break the secondary structure of proteins. Gels were prepared at $15 \%(\mathrm{v} / \mathrm{v})$ final acrylamide concentration (Rotiphorese ${ }^{\circledR}$ Gel 30 (37.5:1), Carl Roth, Karlsruhe, Germany) with 1x resolving gel buffer, $0.1 \%$ SDS (Serva Electrophoresis, Heidelberg, Germany), 0.1\% ammonium persulfate and $0.01 \% \mathrm{~N}, \mathrm{~N}, \mathrm{~N}^{\prime}, \mathrm{N}^{\prime}$, -tetramethylethylendiamin (TEMED) (AppliChem, Darmstadt, Germany). This gel was preceded by a stacking gel at $5 \%(\mathrm{v} / \mathrm{v})$ final acrylamide concentration. Other components were equal to the resolving gel, except for $1 \mathrm{x}$ stacking gel buffer. Gels were embedded in 1x running buffer, and protein samples were loaded in 1x SDS sample buffer after $10 \mathrm{~min}$ incubation at $95^{\circ} \mathrm{C} .2 \mu \mathrm{g}$ protein were added to each well, and PageRuler ${ }^{\mathrm{TM}}$ prestained protein ladder 10-180 kDa (Thermo Fisher Scientific, Waltham, MA, USA) was used as marker. Electrophoresis was run for $1 \mathrm{~h}$ at $10 \mathrm{~mA}$ per gel to let proteins pass the stacking gel. Current was then increased to $20 \mathrm{~mA}$ per gel for $2 \mathrm{~h}$ while proteins passed through the resolving gel. Gels were stained for $1 \mathrm{~h}$ in coomassie staining solution and destained over night in $10 \%$ acetic acid (Merck, Darmstadt, Germany) with multiple solvent changes. Gel images were recorded at the Amersham Imager 600RGB (GE Healthcare, Chicago, IL, USA) in trans illumination mode. 


\subsubsection{Seminative PAGE}

Seminative PAGE was performed under the same conditions as denaturing PAGE with the only difference that protein samples were not heated prior to loading the gel and time in SDS-containing buffer before the gel run was kept as short as possible. DsRed, dTomato and mEGFP were loaded as controls for tetra-, di- and monomeric proteins. Prior to coomassie staining of seminative PAGE gels, fluorescence was recorded at the Amersham Imager 600RGB (GE Healthcare, Chicago, IL, USA) with blue $(460 \mathrm{~nm})$ and green $(520 \mathrm{~nm})$ excitation.

\subsection{Protein characterization}

\subsubsection{In vitro switching of fluorescent proteins}

In vitro switching of fluorescent proteins was facilitated with a mercury-vapor lamp in a quartz glass ultra-micro fluorescence cell cuvette (Hellma, Müllheim, Germany) with a $1.5 \mathrm{~mm}$ light path and $12 \mu \mathrm{l}$ volume. Light from the lamp was redirected with a fiber to the cuvette window and switching was timed by hand. Protein samples were kept at volumes so that the meniscus was not visible through the cuvette window. For switching to the off-state a HQ405/10 X filter was used at $9.9 \mathrm{~mW} / \mathrm{cm}^{2}(0.74 \mathrm{~mW}$ measured behind the cuvette with standard protein buffer). Switching to the on-state was facilitated with a ET500/20 X filter at $18.7 \mathrm{~mW} / \mathrm{cm}^{2}$ (1.40 $\mathrm{mW}$ measured behind the cuvette with standard protein buffer). Powers were measured with a PM200 power meter equipped with a S170C sensor (ThorLabs, Newton, NJ, USA). Illumination duration was $3 \mathrm{~min}$ for both switching to the on- and off-state for all proteins except for Padron, which was switched on for 5 min and switched off for 4 min. Switching duration was determined in preliminary experiments with respect to full saturation of switching.

\subsubsection{Metastability}

Metastability of switching states was measured with the Cary Eclipse fluorescence spectrophotometer (Varian, Palo Alto, CA, USA) in the same cuvette used for switching. Protein samples were diluted in protein buffer to have an absorption of approximately 0.1 at $278 \mathrm{~nm}$, which corresponded to a fluorescent protein concentration of approximately $20 \mu \mathrm{M}$. Diluted samples were equilibrated at $21^{\circ} \mathrm{C}$ in the dark over night and measured the next day at the same temperature. Fluorescence was recorded every 5 min for 310 min with $492 \mathrm{~nm}$ excitation and $516 \mathrm{~nm}$ detection (both with $5 \mathrm{~nm}$ slit). PMT voltage was adjusted so that the on-state signal of the protein was just below PMT saturation. The first value recorded corresponded to the ground-state after equilibration, and samples were then switched between first and second as well as between second and third measurement. Before recording on- or off-state metastability, proteins were switched to the off- or on-state, respectively. Data points were normalized to the first on-state value and averaged. For every protein and measurement 3 repetitions (Padron: 2) were measured on different days. 


\section{Methods}

\subsubsection{Absorption spectra}

To measure absorption spectra of Padron variants, protein samples were diluted in standard protein buffer to have an absorption value of approximately 0.1 at $278 \mathrm{~nm}$, which corresponded to a protein concentration of Padron and related proteins of approximately $20 \mu \mathrm{M}$. Diluted samples were equilibrated over night at $21^{\circ} \mathrm{C}$ in the dark and measured the next day in the same cuvette used for switching. Spectra were recorded for equilibrium, off-, and onstates on a Cary 4000 UV-VIS spectrophotometer (Varian, Palo Alto, CA, USA) between 250 and $650 \mathrm{~nm}$ with 2-3 repetitions on different days. Figures depict single measurements after normalization to $278 \mathrm{~nm}$ absorption and smoothing (Savitzky-Golay, 10 points window size) in OriginPro 2018b (OriginLab Corporation, Northampton, MA, USA). Peak positions were determined from smoothed data with OriginPro 2018b as well, using the peak analyzer function.

\subsubsection{Emission spectra of on- and off-states}

Sample preparation for emission spectra measurements was equal to sample handling for absorption spectra. Spectra were recorded for equilibrium, on- and off-states with the Cary Eclipse fluorescence spectrophotometer (Varian, Palo Alto, CA, USA) at an excitation wavelength of $460 \mathrm{~nm}$. Fluorescence was recorded from 470 to $650 \mathrm{~nm}$ with 2 repetitions of different samples. Graphs depict normalized emission spectra from a single repetition, additional data was in accordance with the spectra shown. Emission peaks of on-state emission spectra were analyzed in OriginPro 2018b (OriginLab Corporation, Northampton, MA, USA) using the descriptive statistics function.

\subsubsection{Extinction coefficient calculation}

The extinction coefficient $\varepsilon$ was calculated from comparative measurements with mEGFP. Absorption spectra of the on-state were recorded as described above. Data was first normalized to the absorption peak at $280 \mathrm{~nm}$ and then smoothed in OriginPro 2018b (OriginLab Corporation, Northampton, MA, USA) (Savitzky-Golay, 10 points window size). Absorption values at the maximum of the deprotonated peak were used for relative calculation of the extinction coefficient as show in equation (5.2). Due to differing primary structures of Padron variants and mEGFP, a correction factor was added based on the extinction coefficient at $280 \mathrm{~nm}$, which was calculated with the ExPASy ProtParam online tool [211]. The extinction coefficient used for mEGFP was $56,000 \mathrm{M}^{-1} \mathrm{~cm}^{-1}$ [189]. 6-7 spectra recorded on different days were used for calculation of the extinction coefficient. 


$$
\varepsilon_{\mathrm{X}}=\frac{A_{\max , \mathrm{X}}}{A_{\max , \mathrm{mEGFP}}} \varepsilon_{\mathrm{mEGFP}} \frac{\varepsilon_{280 \mathrm{~nm}, \mathrm{X}}}{\varepsilon_{280 \mathrm{~nm}, \mathrm{mEGFP}}},
$$

where $\mathrm{X}$ is the protein for which the extiction coefficient $\varepsilon$ is to be calculated. $A_{\max }$ and $A_{280 \mathrm{~nm}}$ are absorption values at the maximum or at $280 \mathrm{~nm}$, respectively.

\subsubsection{Quantum yield calculation}

Quantum yield was calculated relative to Padron by integration of on-state emission spectra that were recorded as described above. Different absorption of Padron variants at the excitation wavelength were considered as shown in equation (5.3). 3-4 on-state emission spectra measured on different days were used for quantum yield calculation. Spectra were integrated in OriginPro 2018b (OriginLab Corporation, Northampton, MA, USA).

$$
Q Y_{\mathrm{X}}=Q Y_{\text {Padron }} \frac{\int_{470 \mathrm{~nm}}^{650 \mathrm{~nm}} F_{\mathrm{X}}(\lambda) d \lambda A_{460 \mathrm{~nm}, \text { Padron }}}{\int_{470 \mathrm{~nm}}^{650} F_{\text {Padron }}(\lambda) d \lambda A_{460 \mathrm{~nm}, \mathrm{X}}}
$$

where $\mathrm{X}$ is the protein for which the quantum yield $Q Y$ is to be calculated, $F(\lambda)$ is the emitted fluorescence with the wavelength $\lambda$ and $A_{460 \mathrm{~nm}}$ is the absorption at the excitation wavelength.

\subsubsection{Molecular Brightness}

Molecular brightness was calculated as the product of extinction coefficient (divided by 1,000) and quantum yield.

\subsubsection{Fluorescence lifetime}

Fluorescence lifetime measurements were performed at concentrations of 25,50 or $100 \mu \mathrm{M}$ in standard protein buffer. Proteins were measured without switching in the equilibrated ground-state with the Quantaurus-Tau fluorescence lifetime spectrometer (Hamamatsu, Hamamatsu, Japan). Samples were measured in a quartz cuvette and measured with $470 \mathrm{~nm}$ excitation. Fluorescence was detected at $516 \mathrm{~nm}$. Typically, 10,000 photons per sample were recorded. The internal response function was measured with Polybead® amino 0.10 micron microspheres (Polysciences, Warrington, PA, USA). Data was analyzed with the associated Quantaurus-Tau software (version 3.0.0.80) with second order fitting.

\subsection{9 $\mathrm{pH}$ dependent absorption, excitation and emission spectra}

$\mathrm{pH}$ dependent absorption, excitation, and emission spectra of the equilibrium state were recorded with the Cytation 3 imaging plate reader (BioTek, Winooski, VT, USA). Samples 


\section{Methods}

were diluted in standard protein buffer to a concentration of $200 \mu \mathrm{M}$ and equilibrated over night. For measurements, triplicate samples were then diluted 1:40 to $5 \mu \mathrm{M}$ with the respective pH buffer in 96 well UV-Star® micro plates (Greiner Bio-One, Frickenhausen, Germany). Absorption spectra were recorded from 250 to $600 \mathrm{~nm}$. Emission spectra were recorded with $470 \mathrm{~nm}$ excitation from 500 to $700 \mathrm{~nm}$, excitation spectra from 400 to $520 \mathrm{~nm}$ with emission detection at $550 \mathrm{~nm}$ while detector sensitivity was scaled to the wells with the highest fluorescence intensity. For $\mathrm{p} K_{a}$ value calculation, fluorescence was recorded with a 485/20 excitation filter and a 528/20 detection filter. Controls were recorded for every buffer in duplicates and subtracted from the data followed by normalization. Excitation and emission spectra peaks were determined in OriginPro 2018b (OriginLab Corporation, Northampton, MA, USA) using the peak analyzer function. $\mathrm{p} K_{a}$ values were calculated by fitting an mono- or biphasic dose response function (monophasic was only used for Padron) as shown in equations (5.4) and (5.5). 3 repetitions (Kohinoor: 2) were measured on different days.

Monophasic dose response function:

$$
F(p H)=C+\frac{A}{1+10^{p K_{a}-p H}}
$$

Biphasic dose response function:

$$
F(p H)=C+\frac{A_{1}}{1+10^{p K_{a 1}-p H}}+\frac{A_{2}}{1+10^{p K_{a 2}-p H}}
$$

where $F$ is the fluorescence intensity dependent from $p H$ and $C$ and $A$ are fitted parameters.

\subsubsection{Size exclusion chromatography}

To address oligomerization tendency of Padron variants, proteins were analyzed via size exclusion chromatography (SEC). Measurements were done with an Äkta pure chromatography system equipped with a Superdex ${ }^{\mathrm{TM}} 200$ Increase 10/300 GL column (GE Healthcare, Chicago, IL, USA). Proteins were diluted to a concentration of $10 \mu \mathrm{M}$ and equilibrated at $6{ }^{\circ} \mathrm{C}$ over night. $250 \mathrm{\mu l}$ protein sample were added to the column and eluted with standard protein buffer at a flow rate of $0.75 \mathrm{ml} / \mathrm{min}$. mEGFP, dTomato, and DsRed were used as markers for different oligomeric states [98, 171, 172]. Proteins were detected with the fixed wavelength UV monitor U9-L (GE Healthcare, Chicago, IL, USA) at $280 \mathrm{~nm}$ independent from fluorescent activity. All SEC experiments were performed at $6{ }^{\circ} \mathrm{C}$ and 3 replications (Kohinoor: 2) were measured on different days. 


\subsection{Microscopy methods}

\subsubsection{Sample preparation}

All microscopy shown in the results section was done with transiently transfected HeLa cells approximately $24 \mathrm{~h}$ post transfection. Coverslips with attached cells were mounted in DMEM without phenol red (Thermo Fisher Scientific, Waltham, MA, USA), which was prepared as described in section 5.1.2.2.

\subsubsection{Optical setup of the RESOLFT microscope}

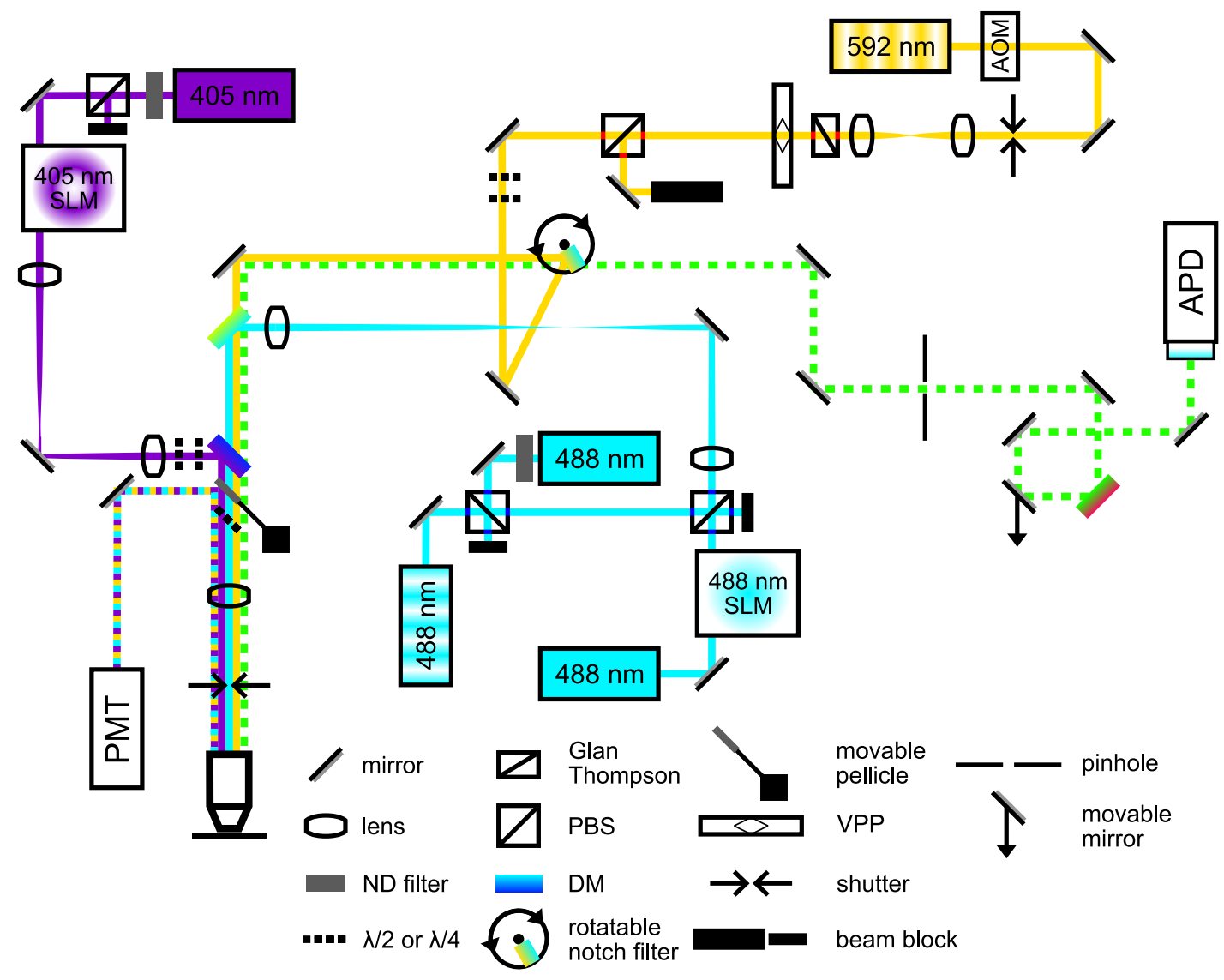

Figure 5.2: Optical setup of the RESOLFT microscope. Laser beam paths (solid lines) and optical parts are shown along with detection paths (dashed lines). Other lasers and optical parts were present but are omitted for clarity as they were not used for experiments presented in this thesis. SLM, spatial light modulator; AOM, acousto optic modulator; APD, avalanche photodiode; PMT, photomultiplier tube; ND, neutral density; PBS, polarized beam splitter; DM, dichroic mirror; VPP, vortex phase plate.

All microscopy data was recorded with a customized 1C RESOLFT QUAD scanning microscope (Abberior Instruments, Göttingen, Germany) and an UPLSAPO 1.4NA 100x oil immersion objective (Olympus, Shinjuku, Japan). The microscope was equipped with 2 continuous wave (CW) $488 \mathrm{~nm} 60 \mathrm{~mW}$ lasers, a CW $405 \mathrm{~nm} 100 \mathrm{~mW}$ laser (all Cobolt, Solna, Sweden), a pulsed $488 \mathrm{~nm} 60 \mathrm{~mW}$ laser (Omicron-Laserage, Rodgau-Dudenhofen, Germany) 


\section{Methods}

and a pulsed $592 \mathrm{~nm}$ Katana $06 \mathrm{HP}>1.2 \mathrm{~W}$ laser (Onefive, Regensdorf, Switzerland). The Katana laser was directed into the beam path with a $594 \mathrm{~nm}$ notch filter that was removed for non-STED applications. During RESOLFT microscopy, one of the CW $488 \mathrm{~nm}$ lasers was used for activation of Padron variants while the other one was attenuated with a neutral density filter and used for fluorescence readout during imaging. Switching of proteins to the off-state in the periphery of the confocal spot was facilitated with the doughnut-shaped $405 \mathrm{~nm}$ laser. The doughnut shape was realized with a spatial light modulator. For STED microscopy, the pulsed $488 \mathrm{~nm}$ laser was used for fluorescence excitation while fluorescence emission was depleted with the $592 \mathrm{~nm}$ laser, which had a doughnut shape facilitated with a VPP-1a phase plate (RPC Photonics, Rochester, NY, USA). Fluorescence was detected with an SPCM-AQRH-13 photon counting module (Excelitas Technologies, Waltham, MA, USA) with a HC 550/88 detection filter for RESOLFT microscopy and with a 525/50 filter for STED microscopy. The detection beam path used for Padron imaging was separated from a red detection path with a dichroic mirror reflecting light with a wavelength below $568 \mathrm{~nm}$ (F48-573 laser beamsplitter H 568 LPXR superflat, AHF, Tübingen-Pfrondorf, Germany). Figure 5.2 depicts the optical parts in detail.

\subsubsection{Power calculation}

In order to estimate the shape of the focal spots and to calculate laser intensities applied, PSFs were probed by imaging $80 \mathrm{~nm}$ gold nanoparticles (BBI Solutions, Crumlin, United Kingdom) mounted in polyvinyl alcohol 4-88 (Sigma-Aldrich, St. Louis, MO, USA). These beads reflected the laser light, which was then recorded with a PMT. 4 equally distributed line profiles through the center of the spot were measured with the Fiji distribution of imageJ (v1.52p, [166, 167]) and fitted in OriginPro 2018b (OriginLab Corporation, Northampton, MA, USA) with a Gaussian nonlinear curve fit. Figure 5.3 depicts the measured PSFs and exemplary line profiles along with the fitted data. FWHM values of all line profiles were averaged and circular shape of the PSF in lateral dimension was assumed for further calculations. Laser power was measured behind the objective with a PM200 power meter equipped with the S170C sensor (ThorLabs, Newton, NJ, USA), and intensities were calculated with equations (5.6) and (5.7).

Gaussian:

$$
I=\frac{P}{\pi\left(\frac{\mathrm{FWHM}}{2}\right)^{2}}
$$

Doughnut-shaped:

$$
I=\frac{P}{2 \pi R \text { FWHM }_{\text {doughnut }}},
$$


where $I$ is the laser intensity, $P$ the power measured behind the objective, FWHM and $F_{W H M}$ doughnut the full-width half maximum of the Gaussian fit of the Gaussian focal spot or the doughnut ring, respectively, and $R$ the radius of the doughnut peak intensity around the doughnut center.
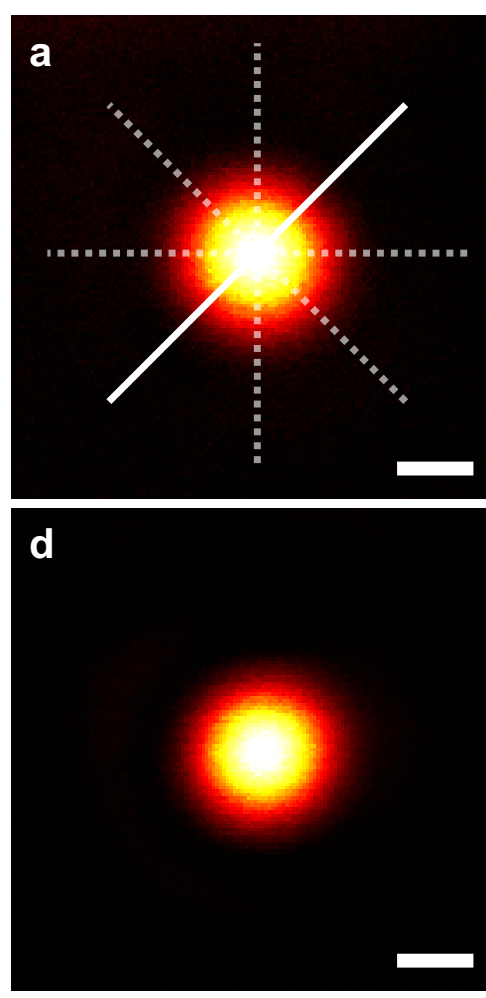
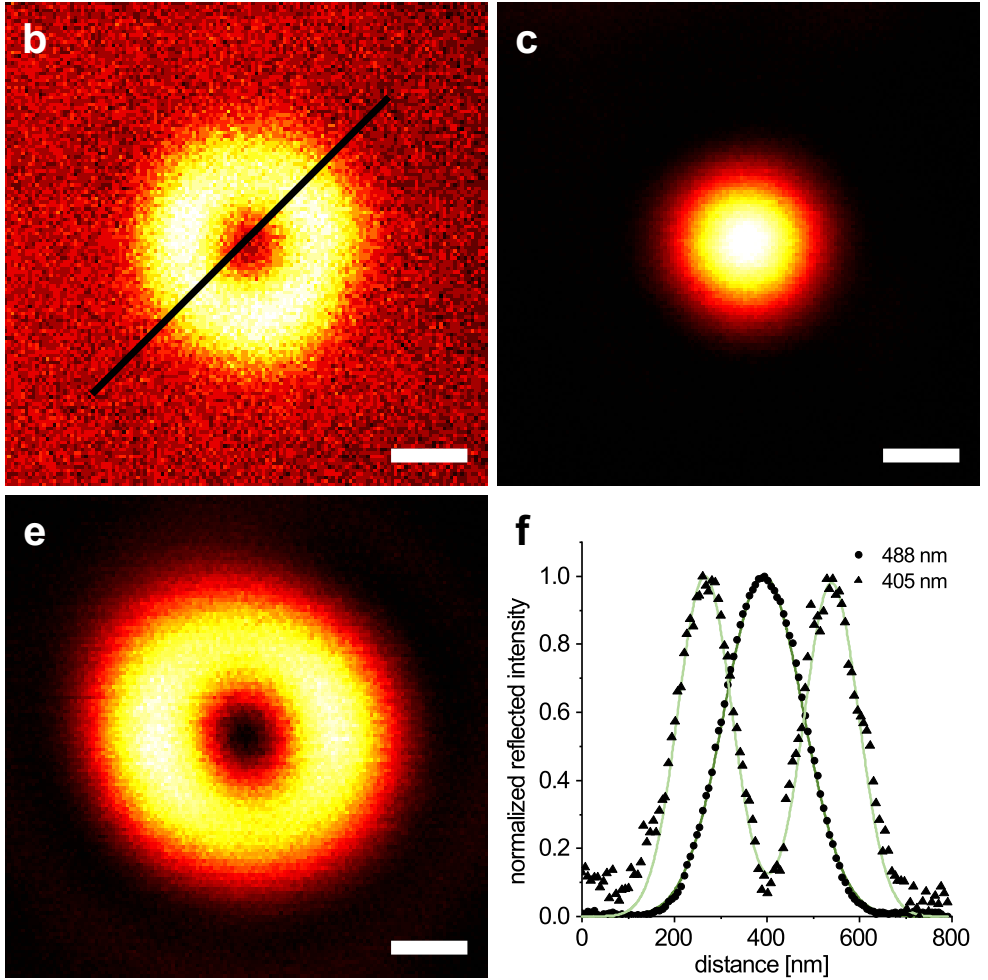

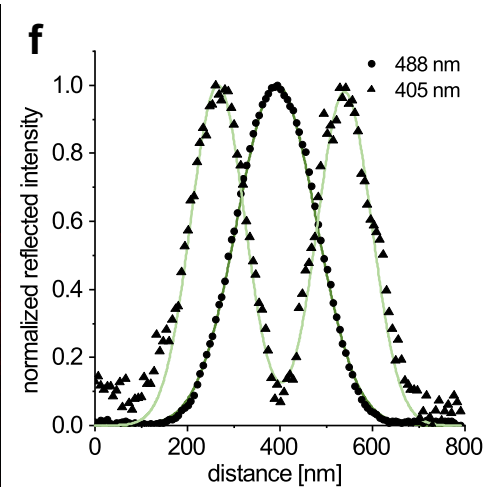

Figure 5.3: Point spread function images of the RESOLFT microscope. PSF images were recorded by probing with $80 \mathrm{~nm}$ gold beads and recording reflected light with a PMT. (a) $488 \mathrm{~nm}$ PSF of the readout laser. Lines show positions of line profiles measured for power calculation and were equal for b-e, solid line marks the line profile shown in f. (b) $405 \mathrm{~nm}$ doughnut PSF, solid line marks the line profile shown in f. (c) $488 \mathrm{~nm}$ PSF of the activation laser. (d) $488 \mathrm{~nm}$ PSF of the pulsed laser. (e) $592 \mathrm{~nm}$ doughnut PSF of the STED laser. (f) Exemplary line profiles of the solid lines in a and b. Scale bars: $150 \mathrm{~nm}$, pixel size was $6.7 \mathrm{~nm}$.

\subsubsection{RESOLFT microscopy}

RESOLFT microscopy was done in three different modes that are described in detail in the results section. In principle, proteins were sequentially switched at every coordinate by applying an initial activation pulse of a CW $488 \mathrm{~nm}$ followed by inactivation of peripheral fluorophores with the doughnut-shaped $405 \mathrm{~nm}$ laser. Residual fluorescence was then recorded with the attenuated $488 \mathrm{~nm}$ laser. Fluorescence readout could be prolonged by simultaneous application of $405 \mathrm{~nm}$ light to prevent peripheral fluorophores from returning to the fluorescent on-state. In a third imaging mode, fluorophore switching was not sequential but rather simultaneous forming a steady-state. Both the attenuated $488 \mathrm{~nm}$ and the $405 \mathrm{~nm}$ laser were active during the full pixel dwell time. This way, a new equilibrium between fluorophores 


\section{Methods}

in the off- and on-state was reached at every new coordinate. Confocal images were always recorded prior to RESOLFT images with equal laser intensities and dwell times but omitting the activation and deactivation steps. Pixel size for all images recorded was $25 \mathrm{~nm}$. Table 5.10 lists the total light doses applied during RESOLFT imaging for the images shown in this thesis.

Table 5.10: Light doses applied for RESOLFT imaging.

\begin{tabular}{|c|c|c|c|c|c|c|c|}
\hline Figure & & 3.25 & 3.27 & 3.28 & 7.10 & 7.11 & 3.30 \\
\hline \multirow{2}{*}{ Pixel dimension } & $\mathrm{x}$ & 568 & 568 & 599 & 661 & 568 & 568 \\
\hline & $\mathbf{y}$ & 431 & 431 & 455 & 503 & 431 & 431 \\
\hline \multirow{3}{*}{$488 \mathrm{~nm}$ activation ${ }^{a}$} & $\mu W$ & 7.57 & 3.91 & - & 3.91 & 7.57 & - \\
\hline & $\mu \mathrm{s}$ & 70 & 50 & - & 100 & 70 & - \\
\hline & $\mathbf{J} / \mathbf{c m}^{2}$ & 84.78 & 31.28 & - & 62.56 & 84.78 & - \\
\hline \multirow{3}{*}{$405 \mathrm{~nm}$ doughnut $^{b}$} & $\mu \mathbf{W}$ & 1.19 & 1.19 & 1.19 & 0.64 & 1.19 & 0.99 \\
\hline & $\mu \mathrm{s}$ & 350 & 490 & 300 & 600 & 470 & 500 \\
\hline & $\mathrm{J} / \mathrm{cm}^{2}$ & 66.64 & 93.3 & 57.12 & 61.44 & 89.49 & 79.2 \\
\hline \multirow{3}{*}{$488 \mathrm{~nm}$ readout $^{c}$} & $\mu \mathrm{W}$ & 0.39 & 0.57 & 0.57 & 0.39 & 0.57 & 0.69 \\
\hline & $\mu \mathrm{s}$ & 120 & 240 & 300 & 80 & 120 & 500 \\
\hline & $\mathrm{J} / \mathbf{c m}^{2}$ & 7.49 & 21.89 & 27.36 & 4.99 & 10.94 & 55.2 \\
\hline
\end{tabular}

\subsubsection{STED microscopy}

STED imaging was done in line step mode with $15 \mu$ s dwell time with the pulsed $488 \mathrm{~nm}$ laser at $5.7 \mathrm{~kW} / \mathrm{cm}^{2}$ for excitation and the $592 \mathrm{~nm}$ STED laser at $4.92 \mathrm{MW} / \mathrm{cm}^{2}$. Confocal images were recorded together with STED images with the latter being recorded with 3 accumulated line steps. Pixel size was $25 \mathrm{~nm}$.

\subsubsection{Image analysis}

All microscopy images were recorded with Imspector (v0.12.10680M, Abberior Instruments, Göttingen, Germany). Images were imported into the Fiji distribution of imageJ (v1.52p, $[166,167])$ by means of the bio-formats plugin [212], and line profiles were measured with a line width of 3 . Line profiles were then fitted in OriginPro 2018b (OriginLab Corporation, Northampton, MA, USA) with a Lorentzian peak function. For bleaching analysis of timelapse recordings, images were imported accordingly as a stack and total counts in a central region of interest were analyzed. Data from 3 different images was normalized and averaged. Microscopy images shown were converted from 16 to 8 bit in imageJ and exported with the "Red Hot" lookup table. 


\section{Material}

\subsection{Buffer and media}

Coomassie staining solution Staining solution for polyacrylamide gels was prepared with $0.05 \%$ (w/v) Coomassie-R250 (Sigma-Aldrich, St. Louis, MO, USA), $25 \%$ (v/v) isopropanol, and $10 \%$ (v/v) acetic acid (both Merck, Darmstadt, Germany). The suspension was stirred for $1 \mathrm{~h}$ and unsolved dye was removed with a filter.

Error-prone dNTP-mix dNTPs for error-prone mutagenesis were prepared as 10x stock solution with $2 \mathrm{mM}$ dATP, $2 \mathrm{mM}$ dGTP, $10 \mathrm{mM}$ dCTP, and $10 \mathrm{mM}$ dTTP (all Thermo Fisher Scientific, Waltham, MA, USA) in water and stored at $-20^{\circ} \mathrm{C}$.

Error-prone PCR buffer PCR-buffer for error-prone mutagenesis was prepared at $\mathrm{pH} 8.3$ as 10x stock solution with $100 \mathrm{mM}$ Tris- $\mathrm{HCl}, 500 \mathrm{mM} \mathrm{KCl,} 70 \mathrm{mM} \mathrm{MgCl}$ (all Merck, Darmstadt, Germany), and $0.1 \%$ (w/v) gelatine (Sigma-Aldrich, St. Louis, MO, USA).

His buffer kit Buffers used for protein purification with the His SpinTrap kit (GE Healthcare, Chicago, IL, USA) were prepared from the stock solutions of the associated His buffer kit (GE Healthcare, Chicago, IL, USA). In detail, all buffers contained $20 \mathrm{mM}$ sodium phosphate and $500 \mathrm{mM}$ sodium chloride at $\mathrm{pH}$ 7.4. Imidazole concentrations varied depending on the buffers function:

- Binding buffer: $20 \mathrm{mM}$ imidazole

- Washing buffer: $50 \mathrm{mM}$ imidazole

- Elution buffer: $500 \mathrm{mM}$ imidazole

LB medium For 1 liter LB-medium, $5 \mathrm{~g} \mathrm{NaCl}$ (Merck, Darmstadt, Germany), $5 \mathrm{~g}$ yeast extract, and $10 \mathrm{~g}$ peptone from casein (both AppliChem, Darmstadt, Germany) were dissolved in sterile water and the $\mathrm{pH}$ value was adjusted with $5 \mathrm{ml} 1 \mathrm{M} \mathrm{NaOH}$ (Merck, Darmstadt, Germany). LB-medium was then autoclaved and stored at room temperature until use. If antibiotics were added to the medium it was stored at $4{ }^{\circ} \mathrm{C}$. For agar plates, $14 \mathrm{~g}$ bacteriology grade agar (AppliChem, Darmstadt, Germany) were added prior to autoclavation, and antibiotics were added afterwards when temperature of the medium was at $60^{\circ} \mathrm{C}$. Agar plates were then casted under laminar flow and stored at $4{ }^{\circ} \mathrm{C}$. For LB-plates used for expression of fluorescent proteins from pBAD constructs $0.02 \%$ (w/v) L-(+)-arabinose (Sigma-Aldrich, St. Louis, MO, USA) were added. Antibiotic concentrations of ampicillin (Sigma-Aldrich, St. Louis, MO, USA) and kanamycin (AppliChem, Darmstadt, Germany) were $50 \mu \mathrm{g} / \mathrm{ml}$. 


\section{Material}

Loading buffer for agarose gels Loading buffer was prepared as $10 \mathrm{ml}$ of a $6 \mathrm{x}$ stock solution with $3 \mathrm{ml}$ glycerol (Merck, Darmstadt, Germany), $15 \mathrm{mg}$ bromphenol blue and $15 \mathrm{mg}$ xylencyanol FF (both AppliChem, Darmstadt, Germany) in 6x TAE-buffer.

PAGE buffers The following buffers and solutions were prepared for polyacrylamide gel electrophoresis (all chemicals were obtained from Merck (Darmstadt, Germany) if not stated otherwise):

- $4 \mathrm{x}$ resolving gel buffer: $1.5 \mathrm{M}$ Tris- $\mathrm{HCl} \mathrm{pH} 8.8$

- 4x stacking gel buffer: $0.5 \mathrm{M}$ Tris- $\mathrm{HCl} \mathrm{pH} 6.8$

- 10x running buffer: $0.25 \mathrm{M}$ Tris, $1.925 \mathrm{M}$ glycine, 1\% SDS (Serva Electrophoresis, Heidelberg, Germany), pH 8.3

- $\mathbf{H}_{2}$ O-saturated isopropanol: $200 \mathrm{ml}$ isopropanol, $100 \mathrm{ml} \mathrm{H}_{2} \mathrm{O}$

- 6x SDS sample buffer: $0.3 \mathrm{M}$ Tris-HCl pH 6.8, $6 \%$ (w/v) SDS (Serva Electrophoresis, Heidelberg, Germany), $6 \%$ (v/v) $\beta$-mercaptoethanol (AppliChem, Darmstadt, Germany), $60 \%$ glycerol, $0.006 \%$ (w/v) bromphenol blue (AppliChem, Darmstadt, Germany)

PCR buffer Standard PCR buffer was prepared at pH 8.3 as 10x stock solution with $100 \mathrm{mM}$ Tris-HCl, $500 \mathrm{mM} \mathrm{KCl}$, and $25 \mathrm{mM} \mathrm{MgCl}_{2}$ (all Merck, Darmstadt, Germany).

pH buffer Buffers used for measurement of $\mathrm{pH}$ spectra were prepared at the concentrations indicated below. $\mathrm{pH}$ values were incremented by 0.5 with an additional buffer at $\mathrm{pH} 10.25$. If not stated differently, chemicals were obtained from Merck (Darmstadt, Germany).

- $\mathbf{p H}$ 3.0-5.5: $100 \mathrm{mM}$ citric acid (Sigma-Aldrich, St. Louis, MO, USA), $150 \mathrm{mM}$ sodium chloride

- pH 6.0-7.0: $100 \mathrm{mM}$ potassium dihydrogen phosphate (Sigma-Aldrich, St. Louis, MO, USA), $150 \mathrm{mM}$ sodium chloride

- pH 7.5-8.5: $100 \mathrm{mM}$ Tris, $150 \mathrm{mM}$ sodium chloride

- pH 9.0-10.5: $100 \mathrm{mM}$ glycine, $150 \mathrm{mM}$ sodium chloride

Phosphate buffered saline Phosphate buffered saline was prepared with $137 \mathrm{mM}$ sodium chloride, $2.68 \mathrm{mM}$ potassium chloride, $8 \mathrm{mM}$ disodium hydrogen phosphate, and $1.47 \mathrm{mM}$ potassium dihydrogen phosphate (all Merck, Darmstadt, Germany). pH was adjusted to 7.4 and PBS was autoclaved until further use. 
Standard protein buffer Purified proteins were stored in standard protein buffer which consisted of $100 \mathrm{mM}$ Tris and $150 \mathrm{mM}$ sodium chloride (both Merck, Darmstadt, Germany) at $\mathrm{pH} 7.5$.

TAE-buffer Tris/acetate/EDTA (TAE) buffer was prepared as 50x stock solution with $2 \mathrm{M}$ Tris(hydroxymethyl)aminomethane (Merck, Darmstadt, Germany), 1 M Sodium acetate trihydrate, and $50 \mathrm{mM}$ EDTA (both AppliChem, Darmstadt, Germany) at pH 7.2. pH was adjusted with acetic acid.

Water Sterile water was filtered with the PURELAB® Classic (ELGA LabWater, High Wycombe, United Kingdom) and autoclaved.

\subsection{Software}

Protein structures were modeled with PyMOL ${ }^{\mathrm{TM}}$ 2.3.0 (Schrödinger, New York, NY, USA). Figures were created with CorelDRAW 2018 (v20.1.0.708, Corel Corporation, Ottawa, Canada) or Affinity Designer for iPad (v1.7.3.1, Serif, Nottingham, UK). All other software used is stated in the respective methods section.

\subsection{Primer}

All DNA oligos used in this thesis were ordered from Sigma-Aldrich (St. Louis, MO, USA), which is now a part of Merck (Darmstadt, Germany).

Table 6.1: Primers used for sequencing of expression plasmids.

\begin{tabular}{cl} 
Primer & Primer sequence \\
\hline 298 & CGGATAACAATTTCACACAG \\
299 & CGAGCGTTCTGAACAAATCC \\
928 & AAATGTCGTAACAACTCCGC \\
1016 & TTCTTGGTAGCAACAGCTACA \\
1639 & ATGCCATAGCATTTTTATCC \\
1640 & GATTTAATCTGTATCAGG \\
2268 & CGCAAATGGGCGGTAGGCGTG \\
4667 & AGCAAGTAAAACCTCTACA \\
7124 & GCAAATGGGCGGTAGGCGTGTAC \\
\hline
\end{tabular}


Table 6.2: Padron mutagenesis primers.

\begin{tabular}{|c|c|c|c|}
\hline Template & Mutagenesis & Primer & Primer sequence \\
\hline \multirow{2}{*}{ pa07 } & \multirow{2}{*}{ F173X SDM } & 2643 & TACCGATGTGACNNNAAAACTACTTAT \\
\hline & & 2644 & ATAAGTAGTTTTNNNGTCACATCGGTA \\
\hline \multirow{2}{*}{ pa22 } & \multirow{2}{*}{ T58X SDM } & 2633 & TATGACATCTTGNNNATGGCGTTCTGT \\
\hline & & 2634 & ACAGAACGCCATNNNCAAGATGTCATA \\
\hline \multirow{2}{*}{ pa38 } & \multirow{2}{*}{ S82X SDM } & 2825 & TATTTCAAGCAGNNNTTTCCTGAGGGCT \\
\hline & & 2826 & AGCCCTCAGGAAANNNCTGCTTGAAATA \\
\hline \multirow{2}{*}{ pa48 } & \multirow{2}{*}{ +GFPend } & 2105 & $\begin{array}{l}\text { ACGGATCCAATGGTGAGCAAGGGCGAGG } \\
\text { AGAACAACATGGCCGTGATTAAACCAGAC }\end{array}$ \\
\hline & & 2161 & $\begin{array}{l}\text { ATTAAGCTTCGAATTCTTACTTGTACAGC } \\
\text { TCGTCCATGGCCTGCCTCGGCAG }\end{array}$ \\
\hline \multirow{2}{*}{ pa57 } & \multirow{2}{*}{ E218G SDM } & 2919 & GAAGCGCATTCTGGGCTGCCGAGGCAG \\
\hline & & 2920 & CTGCCTCGGCAGCCCAGAATGCGCTTC \\
\hline \multirow{2}{*}{ pa60 } & \multirow{2}{*}{ D203D SDM } & 4143 & AGCCACGACAAAGATTACAGTAATGTTA \\
\hline & & 4144 & TAACATTACTGTAATCTTTGTCGTGGCT \\
\hline \multirow{2}{*}{ pa62 } & \multirow{2}{*}{ S190X SDM } & 5511 & CAGACTATCACNNNGTGGACCACCAC \\
\hline & & 5512 & GTGGTGGTCCACNNNGTGATAGTCTG \\
\hline \multirow{2}{*}{ pa69 } & \multirow{2}{*}{ A69X SDM } & 2745 & CTATATTTTCTGGGTATTTNNNGAATACCCTGTTGCCG \\
\hline & & 2746 & CGGCAACAGGGTATTCNNNAAATACCCAGAAAATATAG \\
\hline \multirow{2}{*}{ pa87 } & \multirow{2}{*}{ C69V SDM } & 7360 & GGCAACAGGGTATTCGTGAAATACCCAGAAAAT \\
\hline & & 7361 & ATTTTCTGGGTATTTCACGAATACCCTGTTGCC \\
\hline \multirow{2}{*}{ pa90 } & \multirow{2}{*}{ R66X SDM } & 8155 & TTCTGTTACGGCAACNNNGTATTCGTGAAATAC \\
\hline & & 8156 & GTATTTCACGAATACNNNGTTGCCGTAACAGAA \\
\hline \multirow{2}{*}{ Padron 1.9} & \multirow{2}{*}{ V69X SDM } & 8344 & GGCAACAAGGTATTCNNNAAATACCCAGAAAAT \\
\hline & & 8345 & ATTTTCTGGGTATTTNNNGAATACCTTGTTGCC \\
\hline $\begin{array}{l}\text { Padron } \\
1.9 / 2.0 / 2.1\end{array}$ & Corr. MSM & 9193 & [Phos] GAGAACAACATGGCCGTGATTAAACCAGACATG \\
\hline $\begin{array}{l}\text { Padron } \\
1.9 / 2.0 / 2.1\end{array}$ & Corr. MSM & 9195 & [Phos]CCCGTTCGCGATTGAGGGAGTTGGCCTT \\
\hline $\begin{array}{l}\text { Padron } \\
1.9 / 2.0 / 2.1\end{array}$ & Corr. MSM & 9196 & [Phos]GTCAAAGAAGGCGGACCTCTGCCTTTCGCCTAT \\
\hline Padron 2.1 & Corr. MSM & 9194 & [Phos]CGTATGGAAGGCGCTGTAAATGGACACCCGTTC \\
\hline Padron 2.1 & Corr. MSM & 9197 & [Phos]GGCATTTGTAACGCGACAAACGACATAACCCTG \\
\hline Padron 2.1 & Corr. MSM & 9198 & $\begin{array}{l}\text { [Phos]CCACATTGAGATTAAAAGCCA } \\
\text { CGACAAAGATTACAGT }\end{array}$ \\
\hline
\end{tabular}


Table 6.3: Primers used for cloning of bacterial and mammalian expression constructs. Primer Primer sequence

\begin{tabular}{|c|c|}
\hline 914 & GATCCGCTAGCGCTAATGGTGAGCAAGGGCGAGGAG \\
\hline 915 & CACTCGAGATCTGAGTCCGGACTTGTACAGCTCGTCCATGCC \\
\hline 916 & CTGCAGGTCGACATGGTGAGCAAGGGCGAGGA \\
\hline 1836 & TCCACCGGTCGCCACCATGGTGAGCAAGGGCGAGGAGCTGTTC \\
\hline 1837 & GTCGCGGCCGCTACTTGTACAGCTCGTCCATGCCGAGAG \\
\hline 2244 & TCCACCGGTCGCCACCATGGTGAGCAAGGGCGAGGAG \\
\hline 2291 & GATCTCGAGTGATGGCAGATGAACGGAAAGACGAAGC \\
\hline 2292 & GGTGGATCCTTATCACAAGCCCTGCTTAGCGAGTGCAGC \\
\hline 2884 & CGACGCTAGCATGGTGAGCAAGGGCG \\
\hline 2943 & AGGGCTCGAGCATGGTGAGCAAGGGCGAGGAG \\
\hline 3048 & CCCTGCGGCCGCTTTACTTGTACAGCTCGTCCATGGCCTGCCTCGGCAGC \\
\hline 3978 & CACTCGAGATCTGAGTCCGGACTTGTACAGCTCGTCCATG \\
\hline 4027 & AGGGCTCGAGCATGAGTGTGATTAAACC \\
\hline 4098 & AACGAATTCTTACTTGGCCTGCCT \\
\hline 4552 & CAGATCTCGAGTGGCTGCGTCCGGTCTGGA \\
\hline 4553 & TCCGGTGGATCCTTAGCATTTCAGGCAACTCTCCT \\
\hline 4571 & TCCGCTAGCATGTCTGGGGGCAAAT \\
\hline 4572 & CCGGTGGATCCCGGGCCCGCGGTATTTCTTTCTGCAAGTTGATG \\
\hline 4604 & GTCGCGGCCGCTTACTTGTACAGCTCGTC \\
\hline 4605 & TCCACCGGTCGCCACCATGGTGAGCAAGGGCGAG \\
\hline 5065 & $\begin{array}{l}\text { GACGGTACCGCGGGCCCGGGATCCACCGGTCGCCACCATGG } \\
\text { TGAGCAAGGGCGAGGAG }\end{array}$ \\
\hline 8650 & CCCTGCGGCCGCTTTACTTGTACAGCTCGTCCATGGC \\
\hline 8784 & CCACTCGAGATCTGAGTCCGGACTTGTACAGCTCGTCCATG \\
\hline 9336 & CACTCGAGATCTGAGTCCGGACTTGTACAGCTCGTCCATGGC \\
\hline 9337 & TCCGCTAGCGCTACCGGTCGCCACCATGGTGAGCAAGGGCG \\
\hline 9338 & TTCTGCGGCCGCCTTGTACAGCTCGTCCATGGCCTGCCCC \\
\hline 9340 & AGGGGATCCACCGGTCGCCACCGTGAGCAAGGGCGAGGAGAACAAC \\
\hline 9341 & CGAGCGGCCGCTACTTGTACAGCTCGTCCATGG \\
\hline 9362 & GATCCGCTAGCGCTAATGAGTGTGATTAAACCAGACATGAAGATCAAGC \\
\hline 9363 & CACTCGAGATCTGAGTCCGGACTTGGCCTGCCTCGG \\
\hline 9364 & CTGCAGGTCGACATGAGTGTGATTAAACCAGACATGAAGATCAAGC \\
\hline 9365 & TTCTGCGGCCGCCTTGGCCTGCCTCGGCAG \\
\hline 9366 & TCCACCGGTCGCCACCATGAGTGTGATTAAACCAGACATGAAGATCAAGC \\
\hline 9367 & CCCTGCGGCCGCTTTACTTGGCCTGCCTCGGC \\
\hline 9368 & CGACGCTAGCATGAGTGTGATTAAACCAGACATGAAG \\
\hline 9369 & TCCACCGGTCGCCACCATGAGTGTGATTAAACCAGACATGAAGATC \\
\hline 9370 & GTCGCGGCCGCTTACTTGGCCTGCCTC \\
\hline 9371 & $\begin{array}{l}\text { GACGGTACCGCGGGCCCGGGATCCACCGGTCGCCACCATGAGTGTGATT } \\
\text { AAACCAGACATGAAGATCAAGC }\end{array}$ \\
\hline 9372 & TCCGCTAGCGCTACCGGTCGCCACCATGAGTGTGATTAAACCAGACATGAAG \\
\hline 9373 & CCACTCGAGATCTGAGTCCGGACTTGGCCTGCCTCG \\
\hline 9374 & AGGGGATCCACCGGTCGCCACCAGTGTGATTAAACCAGACATGAAGATCAAGCTGC \\
\hline 9375 & CGAGCGGCCGCTACTTGGCCTGCCTCGG \\
\hline 9376 & CACTCGAGATCTGAGTCCGGACTTGGCCTGCCTCG \\
\hline
\end{tabular}




\section{Appendix}

\subsection{Additional figures}
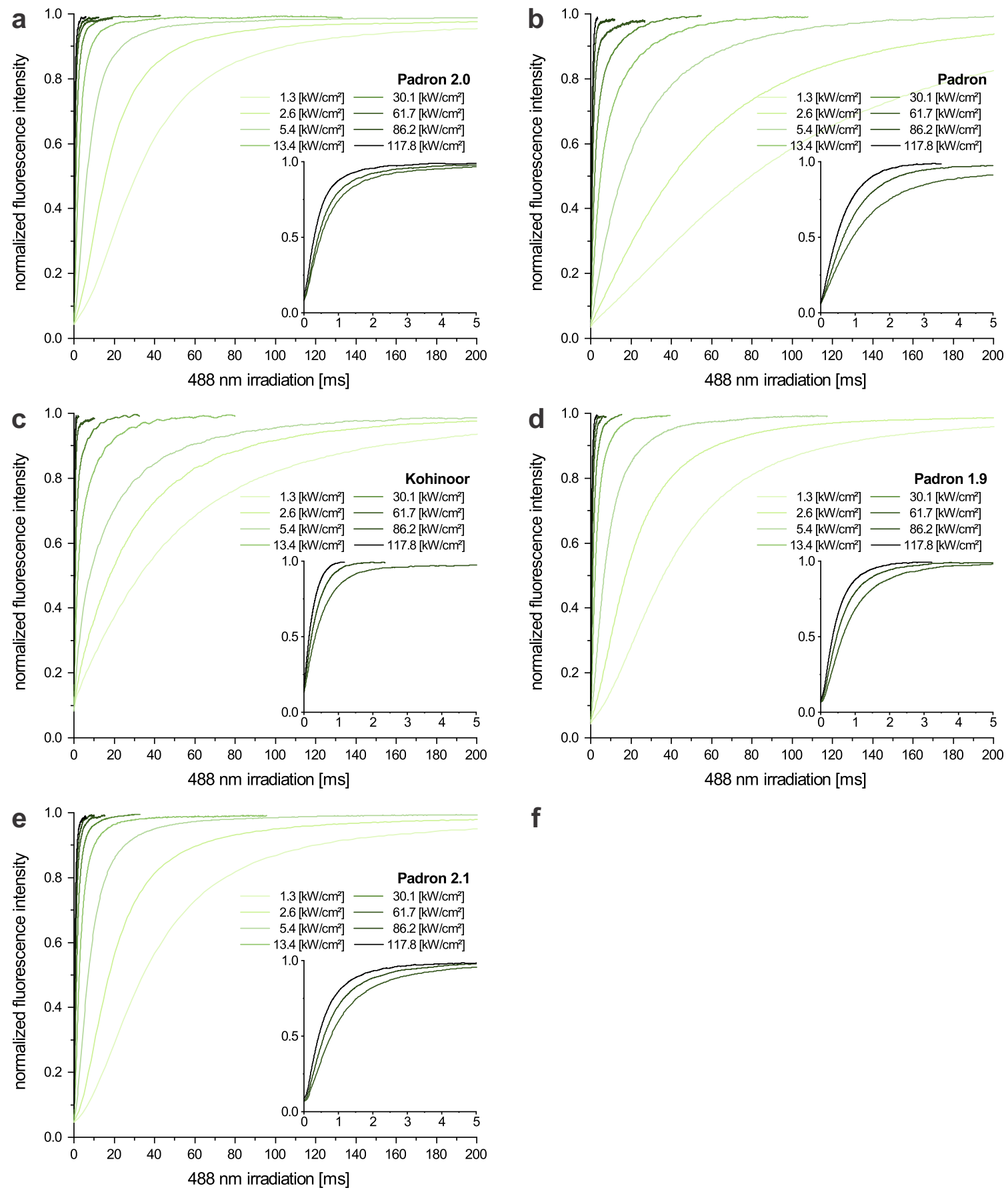

Figure 7.1: Detailed switching kinetics in bacterial colonies. Proteins were switched to $5 \%$ (Kohinoor: 10\%) residual fluorescence and switched back to the on-state with 8 different $488 \mathrm{~nm}$ laser intensities. Insets depict the three highest $488 \mathrm{~nm}$ laser intensities used. (a) Padron 2.0, (b) Padron, (c) Kohinoor, (d) Padron 1.9, (e) Padron 2.1. 

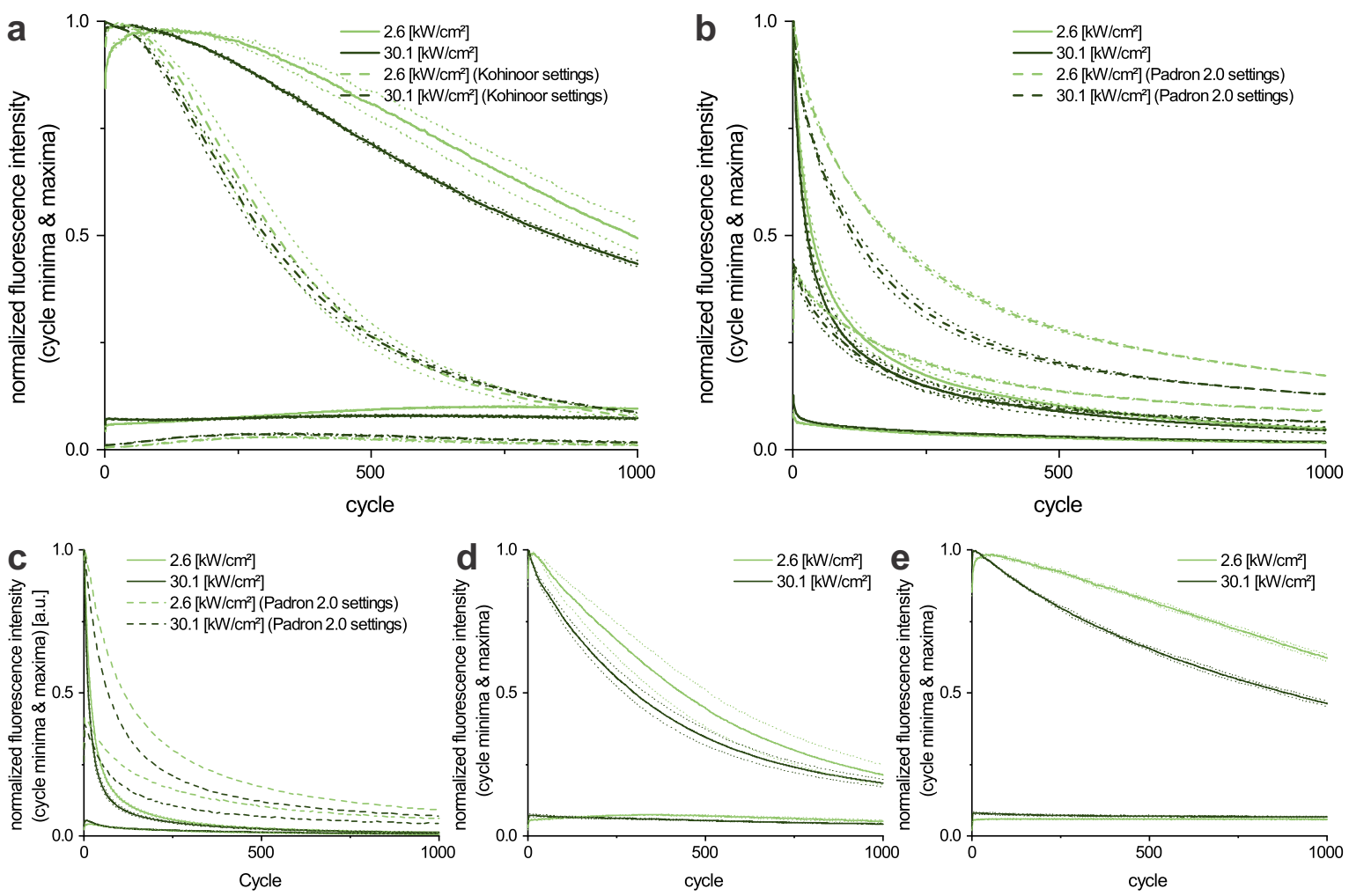

Figure 7.2: Switching fatigue in bacterial colonies with residual off-state intensities. Proteins were switched to $5 \%$ residual off-state fluorescence intensity and back to $95 \%$ of the fluorescent on-state for 1000 times at two different $488 \mathrm{~nm}$ intensities (2.6 and $30.1 \mathrm{~kW} / \mathrm{cm}^{2}$ ). Data for each protein are depicted in single graphs which show on-state fluorescence intensities and residual off-state fluorescence intensity for normal and comparative measurements. (a) Padron 2.0, (b) Kohinoor, (c) Padron, (d) Padron 1.9, (e) Padron 2.1.

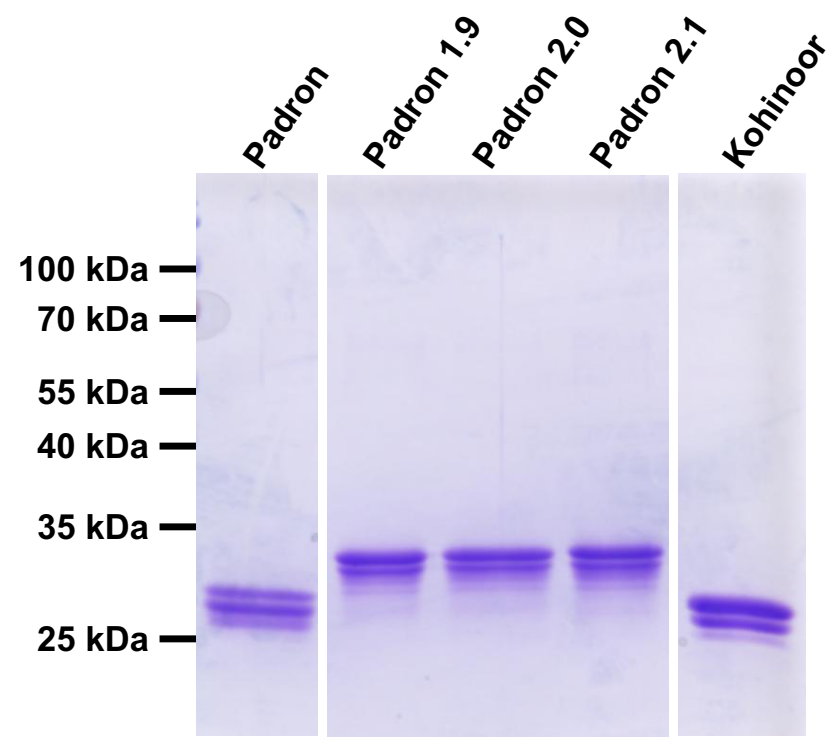

Figure 7.3: SDS PAGE of purified Padron variants. Proteins were isolated as described in section 5.4. Other isolations for measurement repetitions displayed similar purity. 

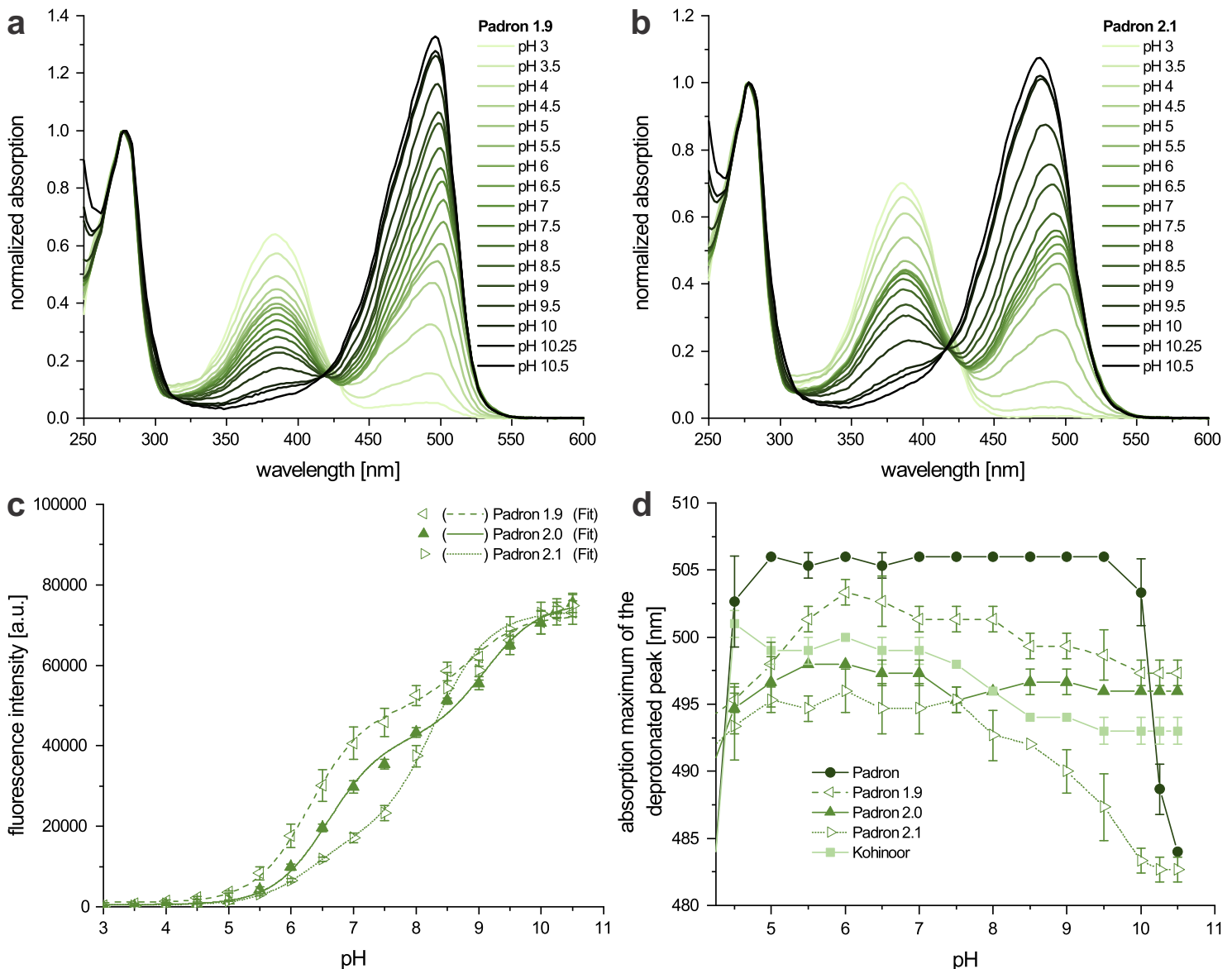

Figure 7.4: pH dependent absorption and fluorescence emission of Padron 1.9 and 2.1. Equilibrium ground-state absorption spectra were measured over a range of $\mathrm{pH}$ values for (a) Padron 1.9 and (b) Padron 2.1. (c) Fluorescence emission from the same samples was measured with a 485/20 excitation and a 528/20 detection filter and results are depicted as a function of $\mathrm{pH}$. Detector sensitivity was scaled to the brightest wells of each sample and Padron 2.0 is shown for comparison. (d) Positions of the deprotonated absorption peaks. 

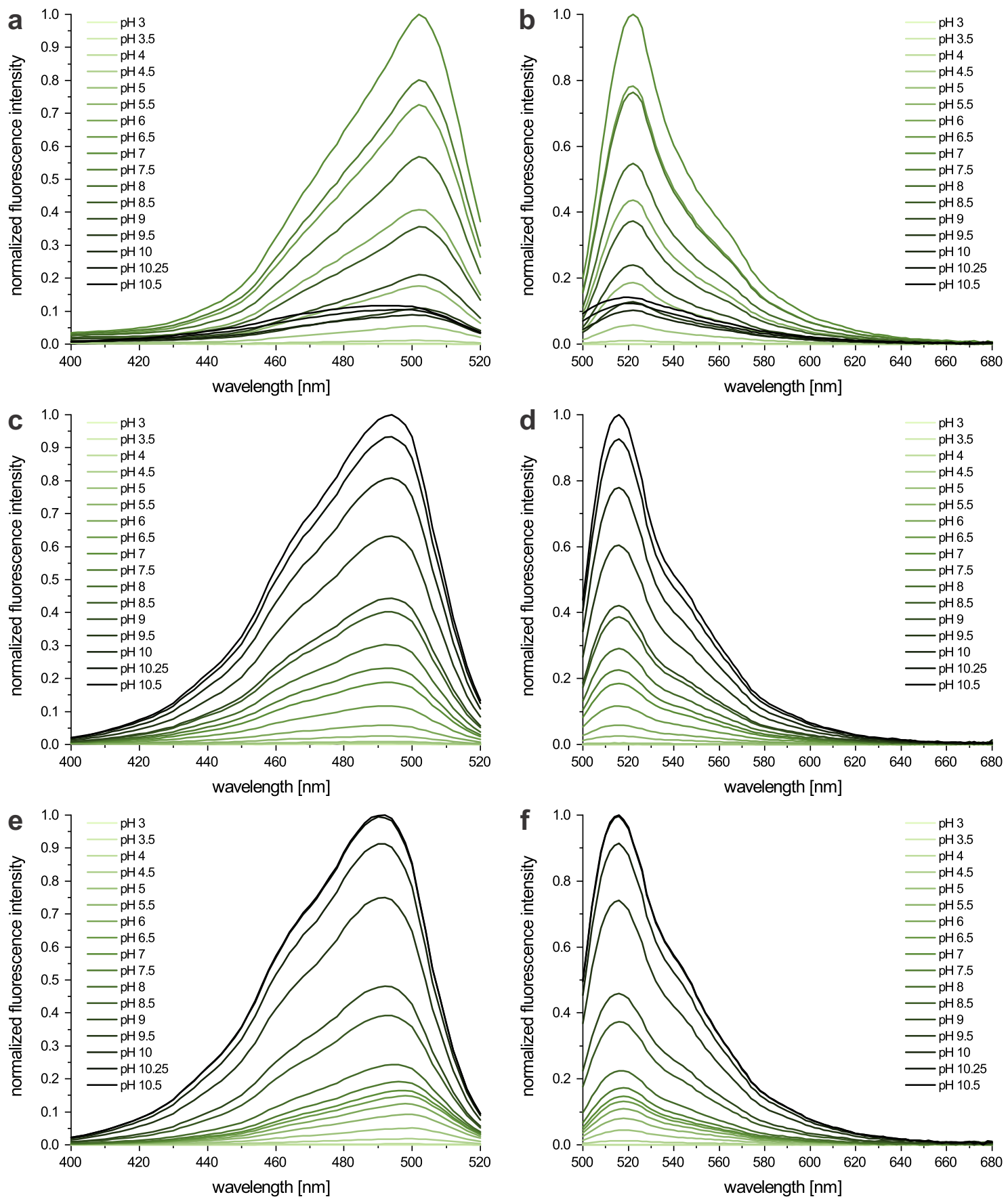

Figure 7.5: pH dependent excitation an emission spectra for Padron, Padron 2.0 and Kohinoor. Equilibrium ground-state excitation and emission spectra were measured over a range of $\mathrm{pH}$ values at $550 \mathrm{~nm}$ detection wavelength and excitation at $470 \mathrm{~nm}$, respectively, for (a,b) Padron, (c,d) Padron 2.0, and (e,f) Kohinoor. 

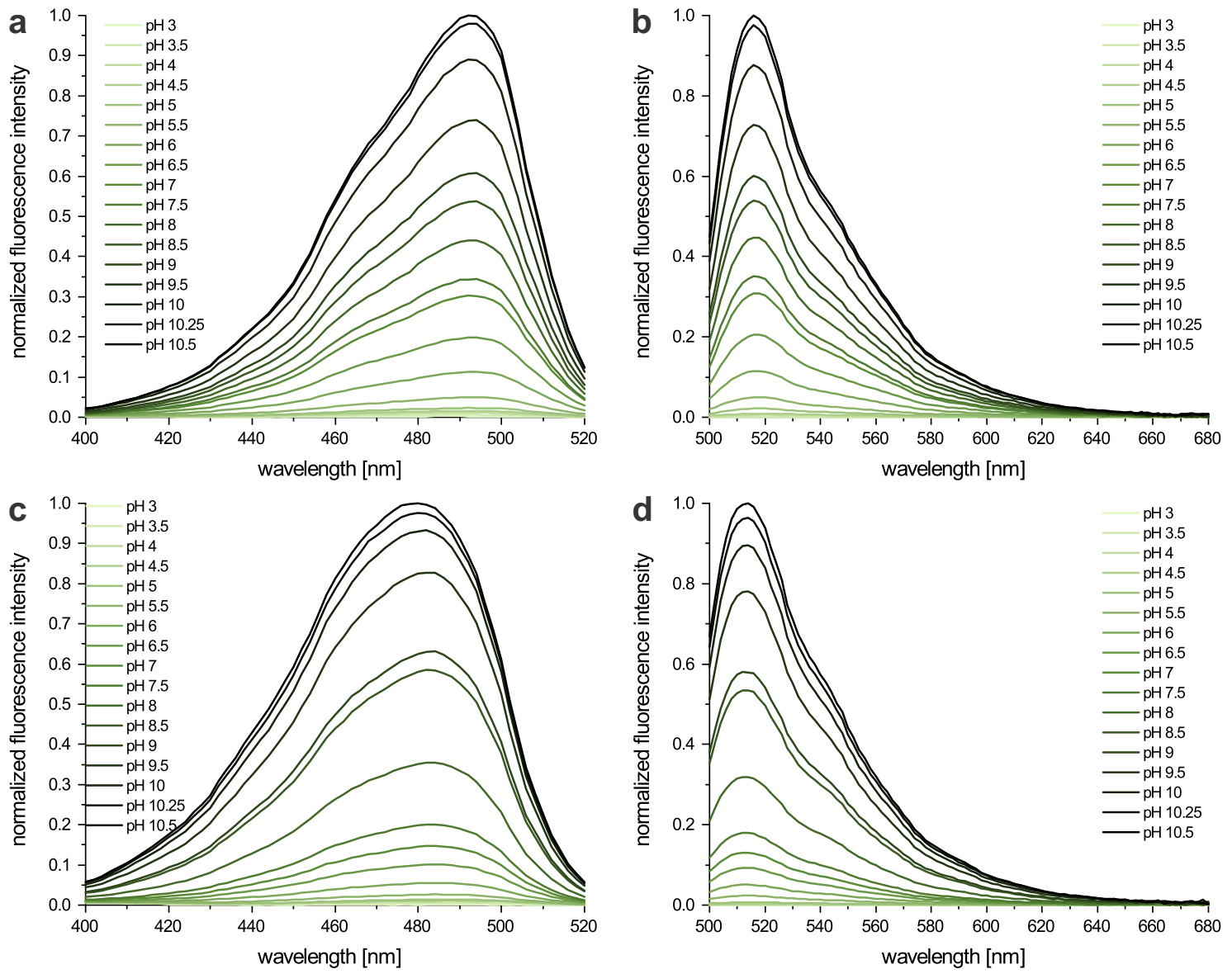

Figure 7.6: $\mathrm{pH}$ dependent excitation an emission spectra for Padron 1.9 and Padron 2.1. Equilibrium ground-state excitation and emission spectra were measured over a range of $\mathrm{pH}$ values at $550 \mathrm{~nm}$ detection wavelength and excitation at $470 \mathrm{~nm}$, respectively, for $(\mathrm{a}, \mathrm{b})$ Padron 1.9 and $(\mathrm{c}, \mathrm{d})$ Padron 2.1. 
a

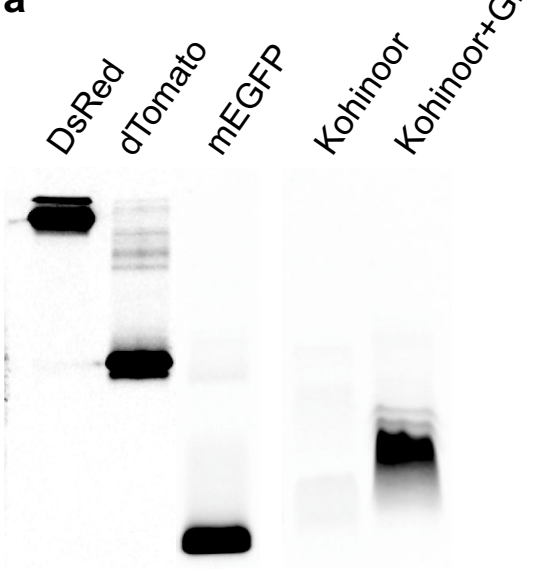

b

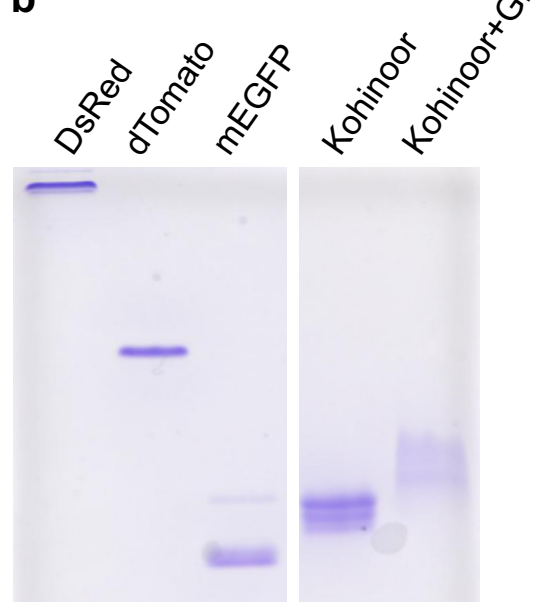

Figure 7.7: Seminative polyacrylamide gel electrophoresis of Kohinoor and Kohinoor+GE. Proteins were analyzed with a seminative PAGE with non-denaturing sample handling prior to application to the gel. DsRed, dTomato, and mEGFP were added as oligomeric state markers for tetrameric, dimeric, and monomeric fluorescent proteins, respectively. (a) Fluorescence image of the gel in the red (DsRed, dTomato) and green (others) channel. (b) Coomassie staining of the same gel. GE, GFPend.
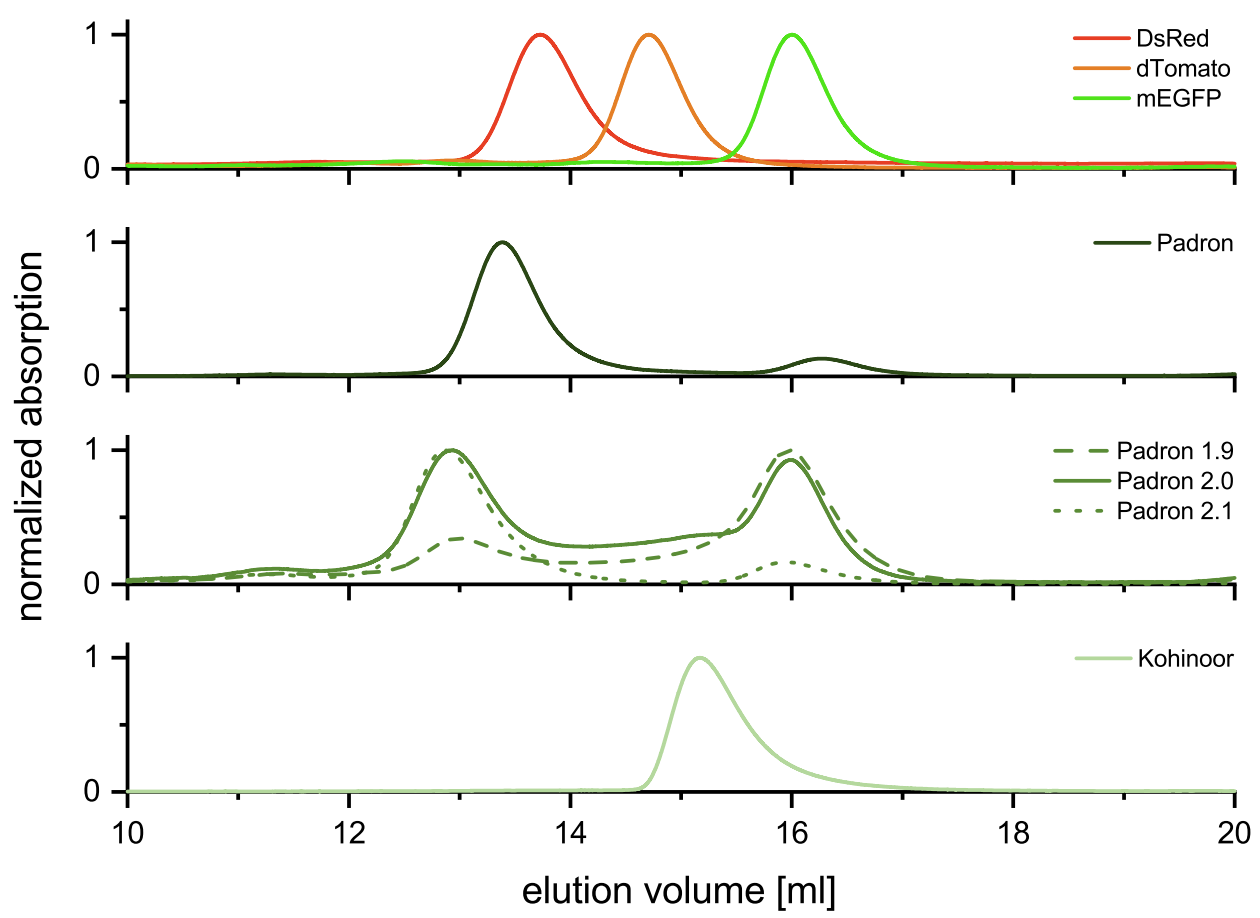

Figure 7.8: Size exclusion chromatography. Protein absorption was detected after size exclusion chromatography (SEC) of protein samples with $10 \mu \mathrm{M}$ concentration without equilibration prior to SEC. Proteins were diluted from concentrations between 300 and $600 \mu \mathrm{M}$. SEC was performed at $4^{\circ} \mathrm{C}$, and DsRed, dTomato, and mEGFP were used as oligomeric markers. Protein absorption was measured at $280 \mathrm{~nm}$. 


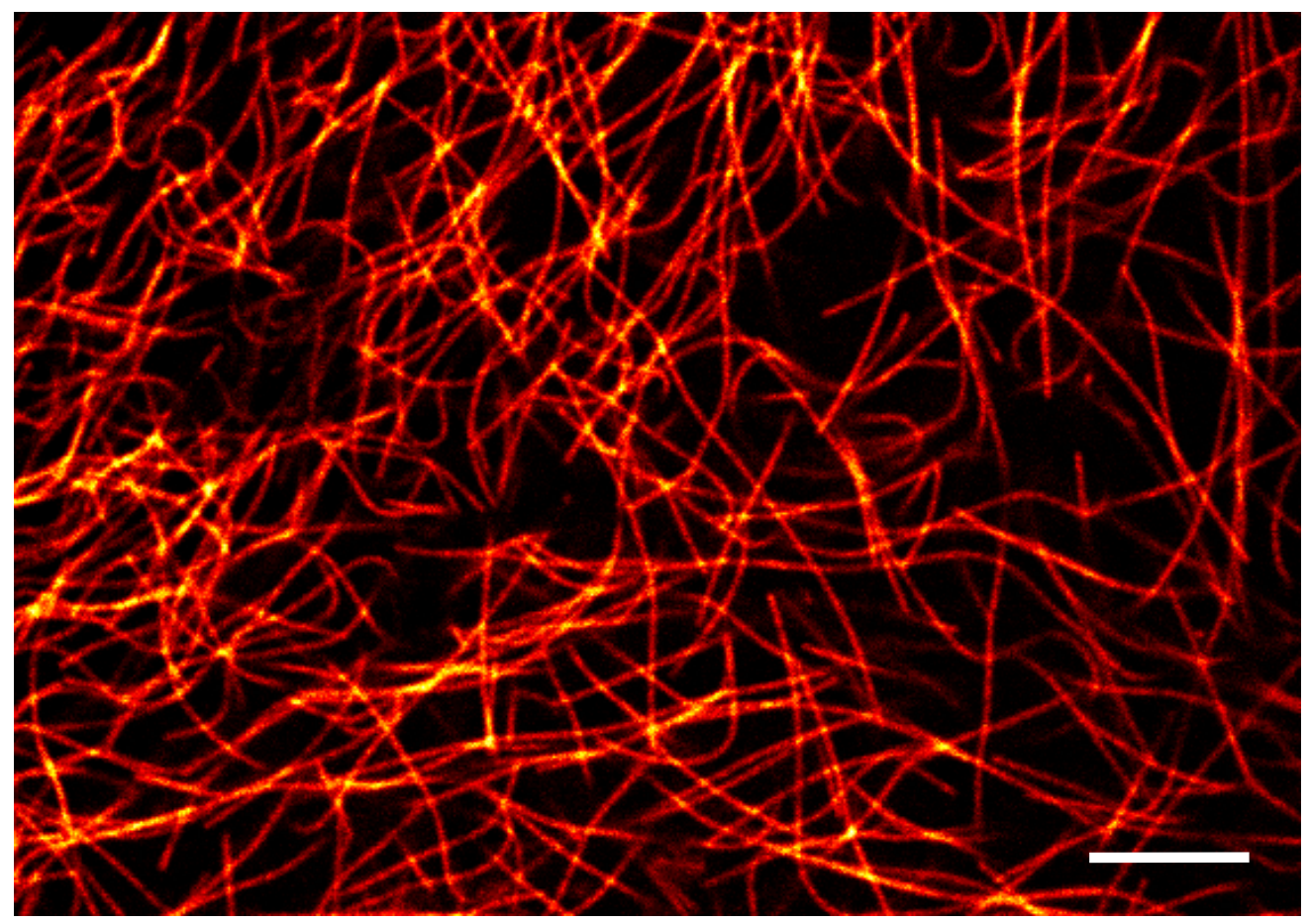

Figure 7.9: Tubulin fusion constructs of resected tissues of third instar larvae of Drosophila melanogaster. Tubulin was directly labeled with Padron0.9, a variant of Padron with an increased tendency for dimerization [140], and integrated into the genome. Images of overexpressed constructs in larvae tissue were recorded in confocal mode. Genetically modified Drosophila melanogaster were created by Dr. Sebastian Schnorrenberg as described in 2016 [153]. Scale bar: $5 \mu \mathrm{m}$. 

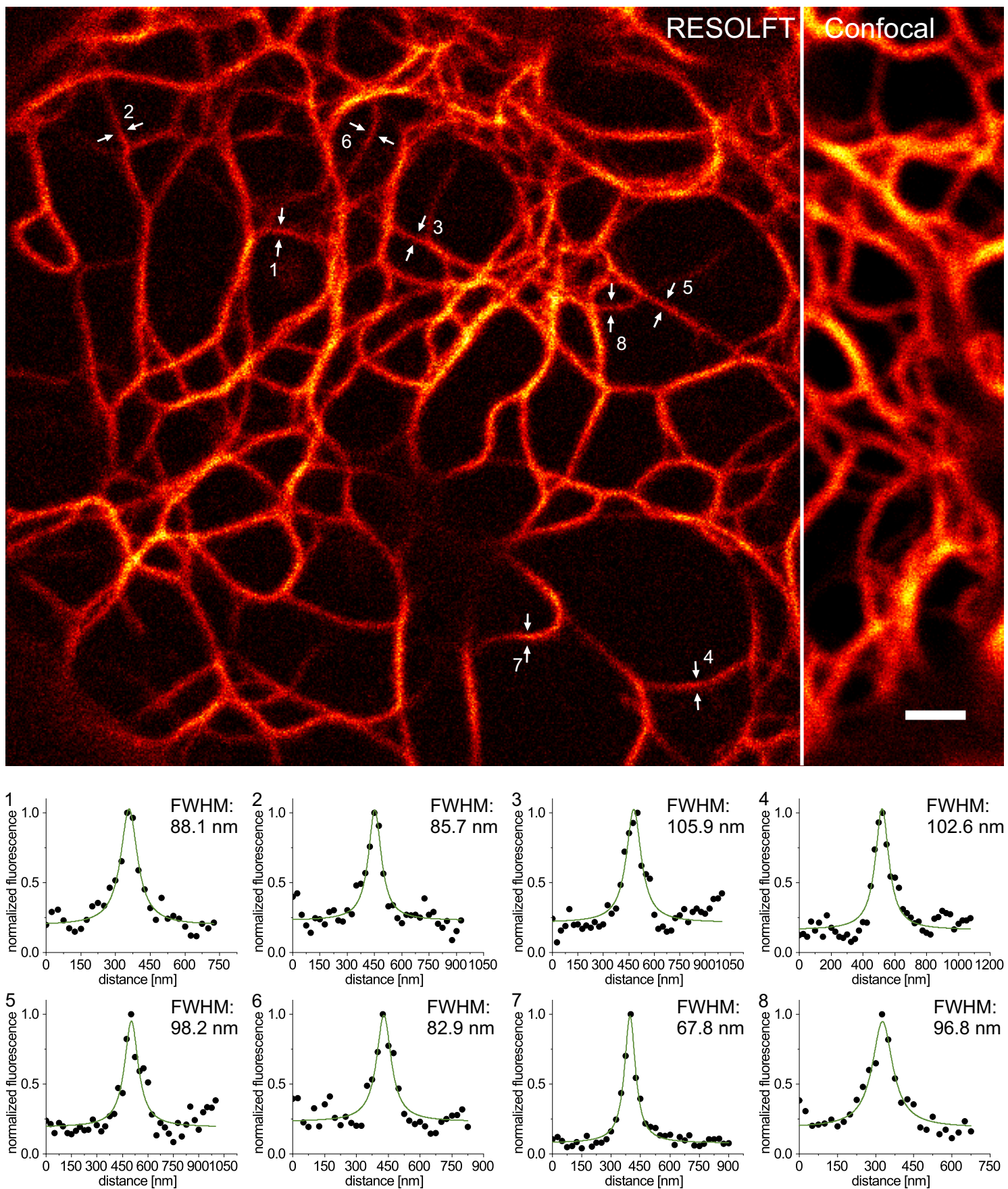

Figure 7.10: RESOLFT microscopy of vimentin-Padron 2.0 fusion constructs with sequential switching sequence. Transiently transfected HeLa cells were imaged with $25 \mathrm{~nm}$ pixel size in pixel step mode. The confocal image was recorded first with $2.1 \mathrm{~kW} / \mathrm{cm}^{2}$ and $20 \mu$ s pixel dwell time. The RESOLFT image was recorded with sequential switching steps with the following pixel dwell times: $100 \mu \mathrm{s} 11.9 \mathrm{~kW} / \mathrm{cm}^{2}$ activation, $50 \mu \mathrm{s}$ break, $600 \mu \mathrm{s}$ $0.6 \mathrm{~kW} / \mathrm{cm}^{2}$ doughnut-shaped beam to switch of peripheral fluorophores, $50 \mu \mathrm{s}$ break, and $80 \mu \mathrm{s} 1.0 \mathrm{~kW} / \mathrm{cm}^{2}$ readout. The sequence was repeated twice for every line and readout fluorescence was accumulated. Line profiles were measured at 8 manually selected positions with 3 adjacent lines and modeled with a Lorentzian fit. FWHM of the fits are indicated in the graphs. Scale bar: $1 \mu \mathrm{m}$. 

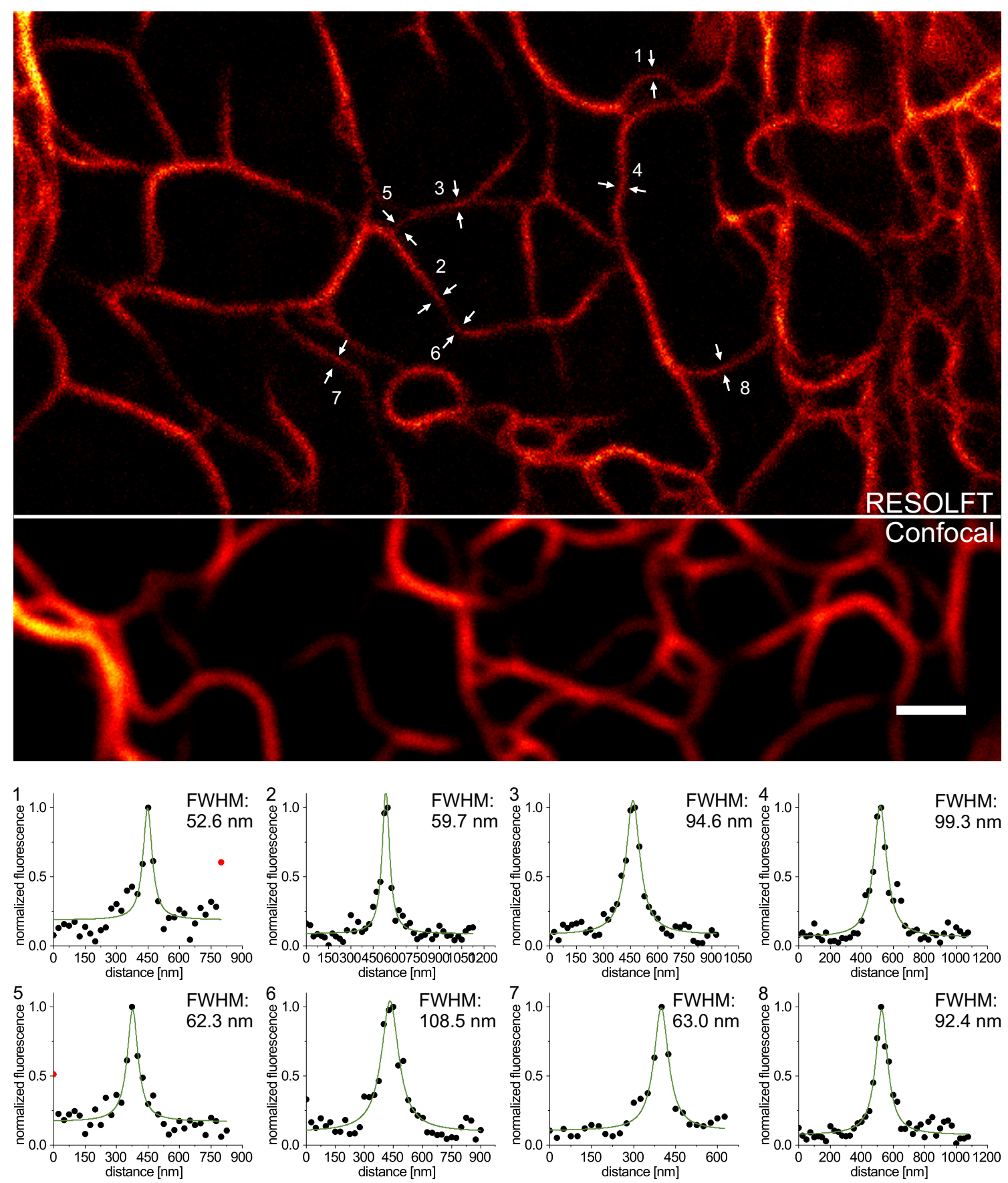

Figure 7.11: RESOLFT microscopy of vimentin-Padron 2.0 fusion constructs with sequential switching sequence and activation suppressed readout. Transiently transfected HeLa cells were imaged with $25 \mathrm{~nm}$ pixel size in pixel step mode. The confocal image was recorded first with $1.4 \mathrm{~kW} / \mathrm{cm}^{2}$ and $240 \mu \mathrm{s} \mathrm{pixel} \mathrm{dwell} \mathrm{time.} \mathrm{The} \mathrm{RESOLFT} \mathrm{image}$ was recorded with sequential switching steps with the following pixel dwell times: $70 \mu \mathrm{s}$ $23.1 \mathrm{~kW} / \mathrm{cm}^{2}$ activation, $350 \mu \mathrm{s} 1.0 \mathrm{~kW} / \mathrm{cm}^{2}$ doughnut-shaped beam to switch of peripheral fluorophores, and $120 \mu \mathrm{s} 1.4 \mathrm{~kW} / \mathrm{cm}^{2}$ readout with simultaneous suppression of fluorescence activation of peripheral fluorophores with the $405 \mathrm{~nm}$ doughnut at $1.0 \mathrm{~kW} / \mathrm{cm}^{2}$. Line profiles were measured at 8 manually selected positions with 3 adjacent lines and modeled with a Lorentzian fit, red data points were excluded from the fit. FWHM of the fits are indicated in the graphs. Scale bar: $1 \mu \mathrm{m}$. 


\subsection{Additional tables}

Table 7.1: Standard deviations [ $\mu \mathrm{s}]$ of switching half-times from off- to on-state.

\begin{tabular}{lccccc} 
kw $/ \mathbf{c m}^{2}$ & Padron & Padron 1.9 & Padron 2.0 & Padron 2.1 & Kohinoor \\
\hline 1.3 & 6,012 & 1,639 & 375 & 1,243 & 2,762 \\
2.6 & 3,153 & 841 & 651 & 558 & 1,164 \\
5.4 & 1,750 & 475 & 208 & 185 & 374 \\
13.4 & 548 & 145 & 124 & 94 & 176 \\
30.1 & 212 & 68 & 40 & 20 & 66 \\
61.7 & 81 & 32 & 51 & 5 & 49 \\
86.2 & 78 & 25 & 47 & 5 & 15 \\
117.8 & 47 & 17 & 25 & 5 & 10 \\
\hline
\end{tabular}

Table 7.2: Standard deviations [us] of switching times to $95 \%$ of the full on-state.

\begin{tabular}{lccccc}
$\mathbf{k w} / \mathbf{c m}^{2}$ & Padron & Padron 1.9 & Padron 2.0 & Padron 2.1 & Kohinoor \\
\hline 1.3 & 20,431 & 4,836 & 4,344 & 7,894 & 23,420 \\
2.6 & 3,717 & 1,972 & 4,514 & 1,655 & 8,870 \\
5.4 & 2,976 & 1,440 & 1,001 & 1,380 & 3,897 \\
13.4 & 1,184 & 505 & 231 & 1,082 & 1,302 \\
30.1 & 1,313 & 287 & 211 & 321 & 265 \\
61.7 & 867 & 92 & 85 & 15 & 401 \\
86.2 & 300 & 79 & 115 & 56 & 68 \\
117.8 & 164 & 52 & 126 & 45 & 37 \\
\hline
\end{tabular}




\section{List of figures}

1.1 Principle of widefield and confocal fluorescence microscopy . . . . . . . . . . 2

1.2 Principle concepts of far-field nanoscopy . . . . . . . . . . . . . 7

1.3 Stimulated emission depletion . . . . . . . . . . . . . . . . . 8

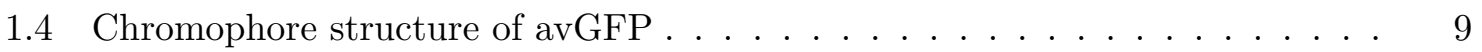

1.5 Crystallographic $\beta$-barrel structure of Padron . . . . . . . . . . . . . . . . 10

1.6 The spectral range of fluorescent proteins . . . . . . . . . . . . . . . . 12

1.7 Reversible switching of Padron . . . . . . . . . . . . . . . . . 15

1.8 Switching characteristics of the positively switching RSFP Padron . . . . . . 17

1.9 RESOLFT principle for a positively switching RSFP . . . . . . . . . . . . 19

1.10 Point-scanning RESOLFT microscopy with Kohinoor . . . . . . . . . . . . . 20

1.11 Influence of irradiation intensity on the switching state of Padron . . . . . . . 21

3.1 Automated bacterial screening with colony selectivity . . . . . . . . . 25

3.2 Improved autofocus for bacterial screening . . . . . . . . . . . . . . . . 27

3.3 Padron mutagenesis . . . . . . . . . . . . . . . . . . . 28

3.4 Protein sequence alignment of new Padron variants and Kohinoor to Padron 29

3.5 Fluorescence intensity and switching half-time of intermediate Padron variants 31

3.6 Residual off-state fluorescence intensity of intermediate Padron variants . . . 32

3.7 Relative on-state fluorescence intensity after 250 switching cycles of intermediate Padron variants . . . . . . . . . . . . . . . . . . 33

3.8 Bright intermediate state of intermediate Padron variants . . . . . . . . . 35

3.9 Saturated residual off-state fluorescence intensity of Padron variants . . . . . 37

3.10 On-Switching kinetics of Padron variants . . . . . . . . . . . . . . . . . . 39

3.11 On-Switching kinetics in bacterial colonies as a function of laser intensity . . 39

3.12 Switching fatigue in bacterial colonies . . . . . . . . . . . . . . . 42

3.13 Photobleaching in bacterial colonies . . . . . . . . . . . . . . . . . 44

3.14 Residual off-state fluorescence intensity with simultaneous 405 and $488 \mathrm{~nm}$ irradiation . . . . . . . . . . . . . . . . . . . . 45

3.15 In vitro metastability of switching states . . . . . . . . . . . . . . . . . . 47

3.16 Absorption spectra of switching states . . . . . . . . . . . . . . . . 49

3.17 Emission spectra of switching states . . . . . . . . . . . . . . 50

3.18 Excitation and emission spectra of the equilibrium ground-state . . . . . . . 51

$3.19 \mathrm{pH}$ dependent absorption and fluorescence emission - Padron, Padron 2.0, and

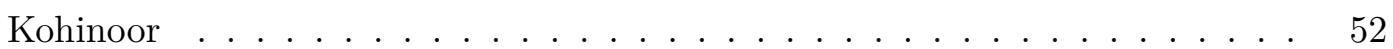

3.20 Seminative polyacrylamide gel electrophoresis . . . . . . . . . . . . . 55

3.21 Size exclusion chromatography _. . . . . . . . . . . . . . . . 57

3.22 Labeling of cellular structures with Padron variants . . . . . . . . . . . . 58 
3.23 STED image of vimentin-Padron 2.0 fusion constructs in transiently transfected HeLa cells . . . . . . . . . . . . . . . . . . . . 62

3.24 RESOLFT microscopy scheme with sequential switching sequence . . . . . . 63

3.25 RESOLFT microscopy of vimentin-Padron 2.0 fusion constructs with sequential switching sequence . . . . . . . . . . . . . . . . . . 64

3.26 RESOLFT microscopy scheme with sequential switching sequence and activation suppressed readout . . . . . . . . . . . . . . 65

3.27 RESOLFT microscopy of vimentin-Padron 2.0 fusion constructs with sequential switching sequence and activation suppressed readout . . . . . . . . 66

3.28 One-step RESOLFT microscopy of vimentin-Padron 2.0 fusion constructs . . 67

3.29 RESOLFT microscopy scheme with one-step RESOLFT . . . . . . . . . . 68

3.30 One-step RESOLFT microscopy of Padron 2.0-Nup50 fusion constructs . . . . 69

3.31 Bleaching of sequential versus one-step RESOLFT microscopy . . . . . . . . . 71

4.1 Mutated positions of new Padron variants in crystallographic structure of Padron 77

4.2 Mutated positions in the chromophore vicinity of Padron . . . . . . . . . 79

4.3 Mutated positions in Padron peripheral to the chromophore . . . . . . . . . 81

4.4 Fluorescence contribution of the bright intermediate state . . . . . . . . . 85

4.5 Fluorescence excitation during switching . . . . . . . . . . . . . . . . . . . . . 89

4.6 Theoretical basis of one-step RESOLFT . . . . . . . . . . . . . . . . . . 92

5.1 Lifeact EGFP pcDNA 3.1(+) plasmid map . . . . . . . . . . . . . . . . . 100

5.2 Optical setup of the RESOLFT microscope . . . . . . . . . . . . . . . 119

5.3 Point spread function images of the RESOLFT microscope . . . . . . . . . 121

7.1 Detailed switching kinetics in bacterial colonies . . . . . . . . . . . . . . . 128

7.2 Switching fatigue in bacterial colonies with residual off-state intensities . . . . 129

7.3 SDS PAGE of purified Padron variants . . . . . . . . . . . . . . . 129

$7.4 \mathrm{pH}$ dependent absorption and fluorescence emission - Padron 1.9, and Padron 2.1130

$7.5 \mathrm{pH}$ dependent excitation an emission spectra for Padron, Padron 2.0, and Kohinoor . . . . . . . . . . . . . . . . . . . 131

7.6 pH dependent excitation an emission spectra for Padron 1.9 and Padron 2.1 . 132

7.7 Seminative polyacrylamide gel electrophoresis of Kohinoor and Kohinoor+GE 133

7.8 Size exclusion chromatography . . . . . . . . . . . . . . . 133

7.9 Tubulin fusion constructs of resected tissues of third instar larvae of Drosophila melanogaster . . . . . . . . . . . . . . . . . 134

7.10 RESOLFT microscopy of vimentin-Padron 2.0 fusion constructs with sequential switching sequence . . . . . . . . . . . . . . . . . 135

7.11 RESOLFT microscopy of vimentin-Padron 2.0 fusion constructs with sequential switching sequence and activation suppressed readout . . . . . . . . 136 


\section{List of tables}

3.1 Switching Background in E. coli . . . . . . . . . . . . . . . . . . . 38

3.2 Switching half-times from off- to on-state . . . . . . . . . . . . . 40

3.3 Switching times to $95 \%$ of the full on-state . . . . . . . . . . . . . 40

3.4 Switching fatigue and photobleaching performance of Padron variants. . . . . 43

3.5 Relative in vitro ground-state fluorescence intensity and ensemble switching background . . . . . . . . . . . . . . . . . . 47

3.6 Spectral properties of Padron variants at $\mathrm{pH} 7.5 \ldots \ldots \ldots \ldots$

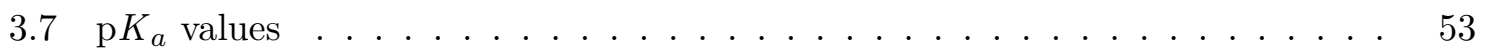

3.8 Extinction coefficients, quantum yields and fluorescence lifetimes of Padron

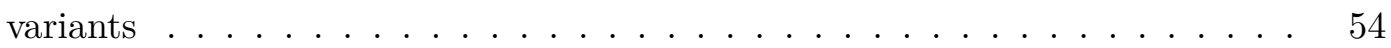

5.1 Primers and restriction enzymes used for cloning of mammalian fusion constructs 99

5.2 PCR amplification PCR cycler protocol . . . . . . . . . . . . . . . 103

5.3 Site-directed mutagenesis PCR cycler protocol . . . . . . . . . . . . . . . . . 104

5.4 Multiple-site mutagenesis PCR cycler protocol . . . . . . . . . . . . . . 104

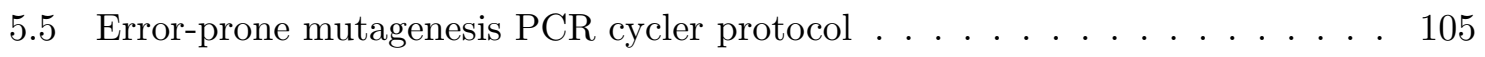

5.6 Padron comparison illumination sequences . . . . . . . . . . . . . . 110

$5.7405 \mathrm{~nm}$ modulation illumination parameters . . . . . . . . . . . . . . 110

5.8 Switching fatigue illumination times f . . . . . . . . . . . . . . . 111

5.9 Residual fluorescence in the off-state with simultaneous $488 \mathrm{~nm}$ irradiation illumination times . . . . . . . . . . . . . . . . . . . 112

5.10 Light doses applied for RESOLFT imaging. . . . . . . . . . . . . . . . 122

6.1 Primers used for sequencing of expression plasmids. . . . . . . . . . . . . 125

6.2 Padron mutagenesis primers. . . . . . . . . . . . . . 126

6.3 Primers used for cloning of bacterial and mammalian expression constructs. . 127

7.1 Standard deviations of switching half-times from off- to on-state . . . . . . 137

7.2 Standard deviations of switching times to $95 \%$ of the full on-state . . . . . 137 


\section{References}

1. Hooke, R. Micrographia, or, Some physiological descriptions of minute bodies made by magnifying glasses (Martyn, J. Allestry, J., London, 1665).

2. Brown, R. A brief account of microscopical observations made in the months of June, July and August 1827, on the particles contained in the pollen of plants; and on the general existence of active molecules in organic and inorganic bodies. Philos Mag 4, 161-73 (1828).

3. Day, C. A., Kraft, L. J., Kang, M. \& Kenworthy, A. K. Analysis of protein and lipid dynamics using confocal fluorescence recovery after photobleaching (FRAP). Curr Protoc Cytom Chapter 2, 2.19.1-29 (2012).

4. Poulon, F., Mehidine, H., Juchaux, M., Varlet, P., Devaux, B., Pallud, J. \& Abi Haidar, D. Optical properties, spectral, and lifetime measurements of central nervous system tumors in humans. Sci Rep 7, 13995 (2017).

5. Testa, I., D'Este, E., Urban, N. T., Balzarotti, F. \& Hell, S. W. Dual channel RESOLFT nanoscopy by using fluorescent state kinetics. Nano Lett 15, 103-6 (2015).

6. Silva, S. F., Domingues, J. P. \& Morgado, A. M. Can we use rapid lifetime determination for fast, fluorescence lifetime based, metabolic imaging? Precision and accuracy of double-exponential decay measurements with low total counts. PLoS One 14, e0216894 (2019).

7. Kodama, Y. Time Gating of Chloroplast Autofluorescence Allows Clearer Fluorescence Imaging In Planta. PLoS One 11, e0152484 (2016).

8. Krause, G. H. \& Weis, E. Chlorophyll fluorescence and photosynthesis: the basics. Annu. Rev. Plant Physiol. Plant Mol Biol 42, 313-49 (1991).

9. Zhou, X., Carranco, R., Vitha, S. \& Hall, T. C. The dark side of green fluorescent protein. New Phytol 168, 313-22 (2005).

10. Griesbeck, O., Baird, G. S., Campbell, R. E., Zacharias, D. A. \& Tsien, R. Y. Reducing the environmental sensitivity of yellow fluorescent protein. Mechanism and applications. J Biol Chem 276, 29188-94 (2001).

11. Denk, W., Strickler, J. H. \& Webb, W. W. Two-photon laser scanning fluorescence microscopy. Science 248, 73-6 (1990).

12. Shcherbakova, D. M., Shemetov, A. A., Kaberniuk, A. A. \& Verkhusha, V. V. Natural photoreceptors as a source of fluorescent proteins, biosensors, and optogenetic tools. Annu Rev Biochem 84, 519-50 (2015).

13. Lecoq, J. \& Schnitzer, M. J. An infrared fluorescent protein for deeper imaging. Nat Biotechnol 29, 715-6 (2011). 
14. Booth, M., Andrade, D., Burke, D., Patton, B. \& Zurauskas, M. Aberrations and adaptive optics in super-resolution microscopy. Microscopy (Oxf) 64, 251-61 (2015).

15. Abbe, E. Beiträge zur Theorie des Mikroskops und der mikroskopischen Wahrnehmung. Archiv für Mikroskopische Anatomie 9, 413-68 (1873).

16. Airy, G. On the Diffraction of an Object-glass with Circular Aperture. Transactions of the Cambridge Philosophical Society 5, 283 (1835).

17. Rayleigh, J. On the theory of optical images, with special reference to the microscope. Philos Mag 42, 167-195 (1896).

18. Freundlich, M. M. Origin of the Electron Microscope. Science 142, 185-8 (1963).

19. Erni, R., Rossell, M. D., Kisielowski, C. \& Dahmen, U. Atomic-resolution imaging with a sub-50-pm electron probe. Phys Rev Lett 102, 096101 (2009).

20. Cox, G. \& Sheppard, C. J. Practical limits of resolution in confocal and non-linear microscopy. Microsc Res Tech 63, 18-22 (2004).

21. Hell, S. W. Far-Field Optical Nanoscopy. Science 316, 1153-8 (2007).

22. Sahl, S. J., Hell, S. W. \& Jakobs, S. Fluorescence nanoscopy in cell biology. Nat Rev Mol Cell Biol 18, 685-701 (2017).

23. Hell, S. W. \& Stelzer, E. H. K. Fundamental improvement of resolution with a 4Piconfocal fluorescence microscope using two-photon excitation. Opt Commun 93, 27782 (1992).

24. Gustafsson, M. G. L., Agard, D. A. \& Sedat, J. W. I ${ }^{5}$ M: 3D widefield light microscopy with better than $100 \mathrm{~nm}$ axial resolution. J Microsc 195, 10-6 (1999).

25. Bewersdorf, J., Schmidt, R. \& Hell, S. W. Comparison of $\mathrm{I}^{5} \mathrm{M}$ and $4 \mathrm{Pi}$-microscopy. $J$ Microsc 222, 105-17 (2006).

26. Gustafsson, M. G. L. Surpassing the lateral resolution limit by a factor of two using structured illumination microscopy. J Microsc 198, 82-7 (2000).

27. Dertinger, T., Colyer, R., Iyer, G., Weiss, S. \& Enderlein, J. Fast, background-free, 3D super-resolution optical fluctuation imaging (SOFI). PNAS 106, 22287-92 (2009).

28. Müller, C. B. \& Enderlein, J. Image scanning microscopy. Phys Rev Lett 104, 198101 (2010).

29. Chen, F., Tillberg, P. W. \& Boyden, E. S. Expansion microscopy. Science 347, 543-8 (2015). 
30. Chen, B. C., Legant, W. R., Wang, K., Shao, L., Milkie, D. E., Davidson, M. W., Janetopoulos, C., Wu, X. S., Hammer J. A., 3., Liu, Z., English, B. P., Mimori-Kiyosue, Y., Romero, D. P., Ritter, A. T., Lippincott-Schwartz, J., Fritz-Laylin, L., Mullins, R. D., Mitchell, D. M., Bembenek, J. N., Reymann, A. C., Böhme, R., Grill, S. W., Wang, J. T., Seydoux, G., Tulu, U. S., Kiehart, D. P. \& Betzig, E. Lattice light-sheet microscopy: imaging molecules to embryos at high spatiotemporal resolution. Science 346, 1257998 (2014).

31. Betzig, E., Patterson, G. H., Sougrat, R., Lindwasser, O. W., Olenych, S., Bonifacino, J. S., Davidson, M. W., Lippincott-Schwartz, J. \& Hess, H. F. Imaging intracellular fluorescent proteins at nanometer resolution. Science 313, 1642-5 (2006).

32. Hess, S. T., Girirajan, T. P. \& Mason, M. D. Ultra-high resolution imaging by fluorescence photoactivation localization microscopy. Biophys J 91, 4258-72 (2006).

33. Rust, M. J., Bates, M. \& Zhuang, X. Sub-diffraction-limit imaging by stochastic optical reconstruction microscopy (STORM). Nat Methods 3, 793-5 (2006).

34. Geisler, C., Schönle, A., von Middendorff, C., Bock, H., Eggeling, C., Egner, A. \& Hell, S. W. Resolution of $\lambda / 10$ in fluorescence microscopy using fast single molecule photo-switching. Applied Physics A 88, 223-6 (2007).

35. Bock, H., Geisler, C., Wurm, C. A., von Middendorff, C., Jakobs, S., Schönle, A., Egner, A., Hell, S. W. \& Eggeling, C. Two-color far-field fluorescence nanoscopy based on photoswitchable emitters. Applied Physics B 88, 161-5 (2007).

36. Egner, A., Geisler, C., von Middendorff, C., Bock, H., Wenzel, D., Medda, R., Andresen, M., Stiel, A. C., Jakobs, S., Eggeling, C., Schönle, A. \& Hell, S. W. Fluorescence nanoscopy in whole cells by asynchronous localization of photoswitching emitters. Biophys J 93, 3285-90 (2007).

37. Fölling, J., Bossi, M., Bock, H., Medda, R., Wurm, C. A., Hein, B., Jakobs, S., Eggeling, C. \& Hell, S. W. Fluorescence nanoscopy by ground-state depletion and single-molecule return. Nat Methods 5, 943-5 (2008).

38. Heilemann, M., van de Linde, S., Schüttpelz, M., Kasper, R., Seefeldt, B., Mukherjee, A., Tinnefeld, P. \& Sauer, M. Subdiffraction-resolution fluorescence imaging with conventional fluorescent probes. Angew Chem Int Ed Engl 47, 6172-6 (2008).

39. Sharonov, A. \& Hochstrasser, R. M. Wide-field subdiffraction imaging by accumulated binding of diffusing probes. PNAS 103, 18911-6 (2006).

40. Hell, S. W. \& Wichmann, J. Breaking the diffraction resolution limit by stimulated emission: stimulated-emission-depletion fluorescence microscopy. Opt Lett 19, 780-2 (1994).

41. Klar, T. A., Jakobs, S., Dyba, M., Egner, A. \& Hell, S. W. Fluorescence microscopy with diffraction resolution barrier broken by stimulated emission. PNAS 97, 8206-10 (2000). 
42. Willig, K. I., Rizzoli, S. O., Westphal, V., Jahn, R. \& Hell, S. W. STED microscopy reveals that synaptotagmin remains clustered after synaptic vesicle exocytosis. Nature 440, 935-9 (2006).

43. Müller, T., Schumann, C. \& Kraegeloh, A. STED microscopy and its applications: new insights into cellular processes on the nanoscale. Chemphyschem 13, 1986-2000 (2012).

44. Hell, S. W., Dyba, M. \& Jakobs, S. Concepts for nanoscale resolution in fluorescence microscopy. Curr Opin Neurobiol 14, 599-609 (2004).

45. Balzarotti, F., Eilers, Y., Gwosch, K. C., Gynnå, A. H., Westphal, V., Stefani, F. D., Elf, J. \& Hell, S. W. Nanometer resolution imaging and tracking of fluorescent molecules with minimal photon fluxes. Science 355, 606-12 (2017).

46. Hell, S. W. \& Kroug, M. Ground-state-depletion fluorescence microscopy: a concept for breaking the diffraction resolutino limit. Applied Physics B 60, 495-7 (1995).

47. Rittweger, E., Wildanger, D. \& Hell, S. W. Far-field fluorescence nanoscopy of diamond color centers by ground state depletion. EPL (Europhysics Letters) 86, 14001 (2009).

48. Hell, S. W. Toward fluorescence nanoscopy. Nat Biotechnol 21, 1347-55 (2003).

49. Bossi, M., Fölling, J., Dyba, M., Westphal, V. \& Hell, S. W. Breaking the diffraction resolution barrier in far-field microscopy by molecular optical bistability. New Journal of Physics 8, 275-275 (2006).

50. Uno, K., Bossi, M. L., Konen, T., Belov, V. N., Irie, M. \& Hell, S. W. Asymmetric Diarylethenes with Oxidized 2-Alkylbenzothiophen-3-yl Units: Chemistry, Fluorescence, and Photoswitching. Advanced Optical Materials 7, 1801746 (2019).

51. Hofmann, M., Eggeling, C., Jakobs, S. \& Hell, S. W. Breaking the diffraction barrier in fluorescence microscopy at low light intensities by using reversibly photoswitchable proteins. PNAS 102, 17565-69 (2005).

52. Prasher, D. C., Eckenrode, V. K., Ward, W. W., Prendergast, F. G. \& Cormier, M. J. Primary structure of the Aequorea victoria green-fluorescent protein. Gene 111, 22933 (1992).

53. Cody, C. W., Prasher, D. C., Westler, W. M., Prendergast, F. G. \& Ward, W. W. Chemical Structure of the Hexapeptide Chromophore of the Aequorea Green-Fluorescent Protein. Biochemistry 32, 1212-8 (1993).

54. Reid, B. G. \& Flynn, G. C. Chromophore Formation in Green Fluorescent Protein. Biochemistry 36, 6786-91 (1997).

55. Zhang, L., Patel, H. N., Lappe, J. W. \& Wachter, R. M. Reaction Progress of Chromophore Biogenesis in Green Fluorescent Protein. J Am Chem Soc 128, 4766-72 (2006).

56. Shimomura, O., Johnson, F. H. \& Saiga, Y. Extraction, Purification and Properties of Aequorin, a Bioluminescent Protein from the Luminous Hydromedusan, Aequorea. $J$ Cell Comp Physiol 59, 223-39 (1962). 
57. Morin, J. G. \& Hastings, J. W. Energy Transfer in a Bioluminescent System. J Cell Physiol 77, 313-8 (1971).

58. Chalfie, M., Tu, Y., Euskirchen, G., Ward, W. W. \& Prasher, D. C. Green Fluorescent Protein as a Marker for Gene Expression. Science 263, 802-5 (1994).

59. Inouye, S. \& Tsuji, F. I. Aequorea green fluorescent protein. Expression of the gene and fluorescence characteristics of the recombinant protein. FEBS Lett 341, 277-80 (1994).

60. Heim, R., Prasher, D. C. \& Tsien, R. Y. Wavelength mutations and posttranslational autoxidation of green fluorescent protein. PNAS 91, 12501-4 (1994).

61. Faro, A. R., Carpentier, P., Jonasson, G., Pompidor, G., Arcizet, D., Demachy, I. \& Bourgeois, D. Low-temperature chromophore isomerization reveals the photoswitching mechanism of the fluorescent protein Padron. J Am Chem Soc 133, 16362-5 (2011).

62. Shimomura, O. Structure of the chromophore of Aequorea green fluorescent protein. FEBS Lett 104, 220-2 (1979).

63. Ormö, M., Cubitt, A. B., Kallio, K., Gross, L. A., Tsien, R. Y. \& Remington, S. J. Crystal Structure of the Aequorea victoria Green Fluorescent Protein. Science 273, 1392-5 (1996).

64. Yang, F., Moss, L. G. \& Phillips, G. N. J. The molecular structure of green fluorescent protein. Nat Biotechnol 14, 1246-51 (1996).

65. Kremers, G. J., Gilbert, S. G., Cranfill, P. J., Davidson, M. W. \& Piston, D. W. Fluorescent proteins at a glance. J Cell Sci 124, 157-60 (2011).

66. Rodriguez, E. A., Campbell, R. E., Lin, J. Y., Lin, M. Z., Miyawaki, A., Palmer, A. E., Shu, X., Zhang, J. \& Tsien, R. Y. The Growing and Glowing Toolbox of Fluorescent and Photoactive Proteins. Trends Biochem Sci 42, 111-129 (2017).

67. Lambert, T. J. FPbase: a community-editable fluorescent protein database. Nat Methods 16, 277-278 (2019).

68. Lambert, G. G., Depernet, H., Gotthard, G., Schultz, D. T., Navizet, I., Lambert, T., Bindels, D. S., Levesque, V., Moffatt, J. N., Salih, A., Royant, A. \& Shaner, N. C. Aequorea victoria's secrets. biorRxiv, doi: 10.1101/677344 (2019).

69. Yarbrough, D., Wachter, R. M., Kallio, K., Matz, M. V. \& Remington, S. J. Refined crystal structure of DsRed, a red fluorescent protein from coral, at 2.0- $\AA$ resolution. PNAS 98, 462-7 (2001).

70. Wachter, R. M., King, B. A., Heim, R., Kallio, K., Tsien, R. Y., Boxer, S. G. \& Remington, S. J. Crystal Structure and Photodynamic Behavior of the Blue Emission Variant Y66H/Y145F of Green Fluorescent Protein. Biochemistry 36, 9759-65 (1997).

71. Niwa, H., Inouye, S., Hirano, T., Matsuno, T., Kojima, S., Kubota, M., Ohashi, M. $\&$ Tsuji, F. I. Chemical nature of the light emitter of the Aequorea green fluorescent protein. PNAS 93, 13617-22 (1996). 
72. Wachter, R. M. Chromogenic Cross-Link Formation in Green Fluorescent Protein. Acc Chem Res 40, 120-7 (2007).

73. Cubitt, A. B., Heim, R., Adams, S. R., Boyd, A. E., Gross, L. A. \& Tsien, R. Y. Understanding, improving and using green fluorescent proteins. Trends Biochem Sci 20, 448-55 (1995).

74. Barondeau, D. P., Tainer, J. A. \& Getzoff, E. D. Structural Evidence for an Enolate Intermediate in GFP Fluorophore Biosynthesis. J Am Chem Soc 128, 3166-8 (2006).

75. Heim, R., Cubitt, A. B. \& Tsien, R. Y. Improved green fluorescence. Nature 373, 663-4 (1995).

76. Barondeau, D. P., Putnam, C. D., Kassmann, C. J., Tainer, J. A. \& Getzoff, E. D. Mechanism and energetics of green fluorescent protein chromophore synthesis revealed by trapped intermediate structures. PNAS 100, 12111-6 (2003).

77. Sniegowski, J. A., Phail, M. E. \& Wachter, R. M. Maturation efficiency, trypsin sensitivity, and optical properties of Arg96, Glu222, and Gly67 variants of green fluorescent protein. Biochem Biophys Res Commun 332, 657-63 (2005).

78. Roldán-Salgado, A., Sánchez-Barreto, C. \& Gaytán, P. LanFP10-A, first functional fluorescent protein whose chromophore contains the elusive mutation G67A. Gene 592, 281-90 (2016).

79. Wood, T. I., Barondeau, D. P., Hitomi, C., Kassmann, C. J., Tainer, J. A. \& Getzoff, E. D. Defining the Role of Arginine 96 in Green Fluorescent Protein Fluorophore Biosynthesis. Biochemistry 44, 13211-20 (2005).

80. Sniegowski, J. A., Lappe, J. W., Patel, H. N., Huffman, H. A. \& Wachter, R. M. Base catalysis of chromophore formation in Arg96 and Glu222 variants of green fluorescent protein. J Biol Chem 280, 26248-55 (2005).

81. Bourgeois, D. Deciphering Structural Photophysics of Fluorescent Proteins by Kinetic Crystallography. Int J Mol Sci 18 (2017).

82. Jung, G., Wiehler, J. \& Zumbusch, A. The photophysics of green fluorescent protein: influence of the key amino acids at positions 65, 203, and 222. Biophys $J \mathbf{8 8}, 1932-47$ (2005).

83. Chattoraj, M., King, B. A., Bublitz, G. U. \& Boxer, S. G. Ultra-fast excited state dynamics in green fluorescent protein: Multiple states and proton transfer. PNAS 93, 8362-7 (1996).

84. Brejc, K., Sixma, T. K., Kitts, P. A., Kain, S. R., Tsien, R. Y., Ormö, M. \& Remington, S. J. Structural basis for dual excitation and photoisomerization of the Aequorea victoria green fluorescent protein. PNAS 94, 2306-11 (1997).

85. Tsien, R. Y. The Green Fluorescent Protein. Annu Rev Biochem 67, 509-44 (1998).

86. Yokoe, H. \& Meyer, T. Spatial dynamics of GFP-tagged proteins investigated by local fluorescence enhancement. Nat Biotechnol 14, 1252-6 (1996). 
87. Day, R. N. \& Davidson, M. W. The fluorescent protein palette: tools for cellular imaging. Chem Soc Rev 38, 2887-921 (2009).

88. Subach, F. V. \& Verkhusha, V. V. Chromophore transformations in red fluorescent proteins. Chem Rev 112, 4308-27 (2012).

89. Dedecker, P., De Schryver, F. C. \& Hofkens, J. Fluorescent proteins: shine on, you crazy diamond. J Am Chem Soc 135, 2387-402 (2013).

90. Dou, J., Vorobieva, A. A., Sheffler, W., Doyle, L. A., Park, H., Bick, M. J., Mao, B., Foight, G. W., Lee, M. Y., Gagnon, L. A., Carter, L., Sankaran, B., Ovchinnikov, S., Marcos, E., Huang, P. S., Vaughan, J. C., Stoddard, B. L. \& Baker, D. De novo design of a fluorescence-activating $\beta$-barrel. Nature 561, 485-491 (2018).

91. Heim, R. \& Tsien, R. Y. Engineering green fluorescent protein for improved brightness, longer wavelengths and fluorescence resonance energy transfer. Curr Biol 6, 178-82 (1996).

92. Ai, H. W., Shaner, N. C., Cheng, Z., Tsien, R. Y. \& Campbell, R. E. Exploration of New Chromophore Structures Leads to the Identification of Improved Blue Fluorescent Proteins. Biochemistry 46, 5904-10 (2007).

93. Wachter, R. M., Elsliger, M. A., Kallio, K., Hanson, G. T. \& Remington, S. J. Structural basis of spectral shifts in the yellow-emission variants of green fluorescent protein. Structure 6, 1267-77 (1998).

94. Matz, M. V., Fradkov, A. F., Labas, Y. A., Savitsky, A. P., Zaraisky, A. G., Markelov, M. L. \& Lukyanov, S. A. Fluorescent proteins from nonbioluminescent Anthozoa species. Nat Biotechnol 17, 969-73 (1999).

95. Strack, R. L., Strongin, D. E., Mets, L., Glick, B. S. \& Keenan, R. J. Chromophore Formation in DsRed Occurs by a Branched Pathway. J Am Chem Soc 132, 8496-505 (2010).

96. Wachter, R. M., Watkins, J. L. \& Kim, H. Mechanistic diversity of red fluorescence acquisition by GFP-like proteins. Biochemistry 49, 7417-27 (2010).

97. Campbell, R. E., Tour, O., Palmer, A. E., Steinbach, P. A., Geoffrey, S. B., Zacharias, D. A. \& Tsien, R. Y. A monomeric red fluorescent protein. PNAS 99, 7877-82 (2002).

98. Shaner, N. C., Campbell, R. E., Steinbach, P. A., Giepmans, B. N., Palmer, A. E. \& Tsien, R. Y. Improved monomeric red, orange and yellow fluorescent proteins derived from Discosoma sp. red fluorescent protein. Nat Biotechnol 22, 1567-72 (2004).

99. Shu, X., Shaner, N. C., Yarbrough, C. A., Tsien, R. Y. \& Remington, S. J. Novel Chromophores and Buried Charges Control Color in mFruits. Biochemistry 45, 963947 (2006).

100. Wang, L., Jackson, W. C., Steinbach, P. A. \& Tsien, R. Y. Evolution of new nonantibody proteins via iterative somatic hypermutation. PNAS 101, 16745-9 (2004). 
101. Shu, X., Wang, L., Colip, L., Kallio, K. \& Remington, S. J. Unique interactions between the chromophore and glutamate 16 lead to far-red emission in a red fluorescent protein. Protein Sci 18, 460-6 (2009).

102. Yoon, E., Konold, P. E., Lee, J., Joo, T. \& Jimenez, R. Far-Red Emission of mPlum Fluorescent Protein Results from Excited-State Interconversion between Chromophore Hydrogen-Bonding States. J Phys Chem Lett 7, 2170-4 (2016).

103. Fischer, A. J. \& Lagarias, J. C. Harnessing phytochrome's glowing potential. PNAS 101, 17334-9 (2004).

104. Murphy, J. T. \& Lagarias, J. C. The phytofluors: a new class of fluorescent protein probes. Curr Biol 7, 870-76 (1997).

105. Ando, R., Hama, H., Yamamoto-Hino, M., Mizuno, H. \& Miyawaki, A. An optical marker based on the UV-induced green-to-red photoconversion of a fluorescent protein. PNAS 99, 12651-6 (2002).

106. Wiedenmann, J., Ivanchenko, S., Oswald, F., Schmitt, F., Röcker, C., Salih, A., Spindler, K. D. \& Nienhaus, G. U. EosFP, a fluorescent marker protein with UVinducible green-to-red fluorescence conversion. PNAS 101, 15905-10 (2004).

107. Gurskaya, N. G., Verkhusha, V. V., Shcheglov, A. S., Staroverov, D. B., Chepurnykh, T. V., Fradkov, A. F., Lukyanov, S. \& Lukyanov, K. A. Engineering of a monomeric green-to-red photoactivatable fluorescent protein induced by blue light. Nat Biotechnol 24, 461-5 (2006).

108. Habuchi, S., Tsutsui, H., Kochaniak, A. B., Miyawaki, A. \& van Oijen, A. M. mKikGR, a monomeric photoswitchable fluorescent protein. PLoS One 3, e3944 (2008).

109. Subach, O. M., Patterson, G. H., Ting, L. M., Wang, Y., Condeelis, J. S. \& Verkhusha, V. V. A photoswitchable orange-to-far-red fluorescent protein, PSmOrange. Nat Methods 8, 771-7 (2011).

110. McEvoy, A. L., Hoi, H., Bates, M., Platonova, E., Cranfill, P. J., Baird, M. A., Davidson, M. W., Ewers, H., Liphardt, J. \& Campbell, R. E. mMaple: a photoconvertible fluorescent protein for use in multiple imaging modalities. PLoS One 7, e51314 (2012).

111. Mizuno, H., Mal, T. K., Tong, K. I., Ando, R., Furata, T., Ikura, M. \& Miyawaki, A. Photo-Induced Peptide Cleavage in the Green-to-Red Conversion of a Fluorescent Protein. Mol Cell 12, 1051-8 (2003).

112. Patterson, G. H. \& Lippincott-Schwartz, J. A Photoactivatable GFP for Selective Photolabeling of Proteins and Cells. Science 297, 1873-7 (2002).

113. Subach, F. V., Patterson, G. H., Manley, S., Gillette, J. M., Lippincott-Schwartz, J. \& Verkhusha, V. V. Photoactivatable mCherry for high-resolution two-color fluorescence microscopy. Nat Methods 6, 153-9 (2009). 
114. Subach, F. V., Patterson, G. H., Renz, M., Lippincott-Schwartz, J. \& Verkhusha, V. V. Bright Monomeric Photoactivatable Red Fluorescent Protein for Two-Color SuperResolution sptPALM of Live Cells. J Am Chem Soc 132, 6481-91 (2010).

115. Gunewardene, M. S., Subach, F. V., Gould, T. J., Penoncello, G. P., Gudheti, M. V., Verkhusha, V. V. \& Hess, S. T. Superresolution imaging of multiple fluorescent proteins with highly overlapping emission spectra in living cells. Biophys $J$ 101, 1522-8 (2011).

116. Henderson, J. N., Gepshtein, R., Heenan, J. R., Kallio, K., Huppert, D. \& Remington, S. J. Structure and Mechanism of the Photoactivatable Green Fluorescent Protein. $J$ Am Chem Soc 131, 4176-77 (2009).

117. Bourgeois, D. \& Adam, V. Reversible photoswitching in fluorescent proteins: a mechanistic view. IUBMB Life 64, 482-91 (2012).

118. Lukyanov, K. A., Fradkov, A. F., Gurskaya, N. G., Matz, M. V., Labas, Y. A., Savitsky, A. P., Markelov, M. L., Zaraisky, A. G., Zhao, X., Fang, Y., Tan, W. \& Lukyanov, S. A. Natural animal coloration can Be determined by a nonfluorescent green fluorescent protein homolog. J Biol Chem 275, 25879-82 (2000).

119. Ando, R., Mizuno, H. \& Miyawaki, A. Regulated Fast Nucleocytoplasmic Shuttling Observed by Reversible Protein Highlighting. Science 306, 1370-3 (2004).

120. Andresen, M., Stiel, A. C., Fölling, J., Wenzel, D., Schönle, A., Egner, A., Eggeling, C., Hell, S. W. \& Jakobs, S. Photoswitchable fluorescent proteins enable monochromatic multilabel imaging and dual color fluorescence nanoscopy. Nat Biotechnol 26, 1035-40 (2008).

121. Tiwari, D. K., Arai, Y., Yamanaka, M., Matsuda, T., Agetsuma, M., Nakano, M., Fujita, K. \& Nagai, T. A fast- and positively photoswitchable fluorescent protein for ultralow-laser-power RESOLFT nanoscopy. Nat Methods 12, 515-8 (2015).

122. Stiel, A. C., Andresen, M., Bock, H., Hilbert, M., Schilde, J., Schönle, A., Eggeling, C., Egner, A., Hell, S. W. \& Jakobs, S. Generation of monomeric reversibly switchable red fluorescent proteins for far-field fluorescence nanoscopy. Biophys J 95, 2989-97 (2008).

123. Grotjohann, T., Testa, I., Leutenegger, M., Bock, H., Urban, N. T., Lavoie-Cardinal, F., Willig, K. I., Eggeling, C., Jakobs, S. \& Hell, S. W. Diffraction-unlimited all-optical imaging and writing with a photochromic GFP. Nature 478, 204-8 (2011).

124. Grotjohann, T., Testa, I., Reuss, M., Brakemann, T., Eggeling, C., Hell, S. W. \& Jakobs, S. rsEGFP2 enables fast RESOLFT nanoscopy of living cells. eLife 1, e00248 (2012).

125. Stiel, A. C., Trowitzsch, S., Weber, G., Andresen, M., Eggeling, C., Hell, S. W., Jakobs, S. \& Wahl, M. C. $1.8 \AA$ bright-state structure of the reversibly switchable fluorescent protein Dronpa guides the generation of fast switching variants. Biochem $J$ 402, 35-42 (2007). 
126. Ando, R., Flors, C., Mizuno, H., Hofkens, J. \& Miyawaki, A. Highlighted generation of fluorescence signals using simultaneous two-color irradiation on Dronpa mutants. Biophys J 92, L97-9 (2007).

127. Zhang, X., Chen, X., Zeng, Z., Zhang, M., Sun, Y., Xi, P., Peng, J. \& Xu, P. Development of a Reversibly Switchable Fluorescent Protein for Super-Resolution Optical Fluctuation Imaging (SOFI). ACS Nano 9, 2659-67 (2015).

128. Zhang, X., Zhang, M., Li, D., He, W., Peng, J., Betzig, E. \& Xu, P. Highly photostable, reversibly photoswitchable fluorescent protein with high contrast ratio for live-cell superresolution microscopy. PNAS 113, 10364-9 (2016).

129. Pennacchietti, F., Serebrovskaya, E. O., Faro, A. R., Shemyakina, I., Bozhanova, N. G., Kotlobay, A. A., Gurskaya, N. G., Bodén, A., Dreier, J., Chudakov, D. M., Lukyanov, K. A., Verkhusha, V. V., Mishin, A. S. \& Testa, I. Fast reversibly photoswitching red fluorescent proteins for live-cell RESOLFT nanoscopy. Nat Methods 15, 601-604 (2018).

130. Adam, V., Moeyaert, B., David, C. C., Mizuno, H., Lelimousin, M., Dedecker, P., Ando, R., Miyawaki, A., Michiels, J., Engelborghs, Y. \& Hofkens, J. Rational design of photoconvertible and biphotochromic fluorescent proteins for advanced microscopy applications. Chem Biol 18, 1241-51 (2011).

131. Adam, V., Lelimousin, M., Boehme, S., Desfonds, G., Nienhaus, K., Field, M. J., Wiedenmann, J., McSweeney, S., Nienhaus, G. U. \& Bourgeois, D. Structural characterization of IrisFP, an optical highlighter undergoing multiple photo-induced transformations. PNAS 105, 18343-8 (2008).

132. Duan, C., Adam, V., Byrdin, M. \& Bourgeois, D. in Photoswitching Proteins: Methods and Protocols (ed Cambridge, S.) 177-202 (Springer New York, New York, NY, 2014).

133. Habuchi, S., Ando, R., Dedecker, P., Verheijen, W., Mizuno, H., Miyawaki, A. \& Hofkens, J. Reversible single-molecule photoswitching in the GFP-like fluorescent protein Dronpa. PNAS 102, 9511-6 (2005).

134. Chang, J., Romei, M. G. \& Boxer, S. G. Structural Evidence of Photoisomerization Pathways in Fluorescent Proteins. J Am Chem Soc 141, 15504-15508 (2019).

135. Petersen, J., Wilmann, P. G., Beddoe, T., Oakley, A. J., Devenish, R. J., Prescott, M. \& Rossjohn, J. The 2.0- $\AA$ crystal structure of eqFP611, a far red fluorescent protein from the sea anemone Entacmaea quadricolor. J Biol Chem 278, 44626-31 (2003).

136. Violot, S., Carpentier, P., Blanchoin, L. \& Bourgeois, D. Reverse pH-Dependence of Chromophore Protonation Explains the Large Stokes Shift of the Red Fluorescent Protein mKeima. J Am Chem Soc 131, 10356-7 (2009).

137. Shinoda, H., Lu, K., Nakashima, R., Wazawa, T., Noguchi, K., Matsuda, T. \& Nagai, T. Acid-Tolerant Reversibly Switchable Green Fluorescent Protein for Super-resolution Imaging under Acidic Conditions. Cell Chem Biol (2019). 
138. Wilmann, P. G., Turcic, K., Battad, J. M., Wilce, M. C., Devenish, R. J., Prescott, M. \& Rossjohn, J. The $1.7 \AA$ crystal structure of Dronpa: a photoswitchable green fluorescent protein. J Mol Biol 364, 213-24 (2006).

139. Andresen, M., Stiel, A. C., Trowitzsch, S., Weber, G., Eggeling, C., Wahl, M. C., Hell, S. W. \& Jakobs, S. Structural basis for reversible photoswitching in Dronpa. PNAS 104, 13005-9 (2007).

140. Brakemann, T., Weber, G., Andresen, M., Groenhof, G., Stiel, A. C., Trowitzsch, S., Eggeling, C., Grubmüller, H., Hell, S. W., Wahl, M. C. \& Jakobs, S. Molecular basis of the light-driven switching of the photochromic fluorescent protein Padron. J Biol Chem 285, 14603-9 (2010).

141. Yang, J. S., Huang, G. J., Liu, Y. H. \& Peng, S. M. Photoisomerization of the green fluorescence protein chromophore and the meta- and para-amino analogues. Chem Commun (Camb), 1344-6 (2008).

142. Mizuno, H., Mal, T. K., Wälchli, M., Kikuchi, A., Fukano, T., Ando, R., Jeyakanthan, J., Taka, J., Shiro, Y., Ikura, M. \& Miyawaki, A. Light-dependent regulation of structural flexibility in a photochromic fluorescent protein. PNAS 105, 9227-32 (2008).

143. Mizuno, H., Mal, T. K., Wälchli, M., Fukano, T., Ikura, M. \& Miyawaki, A. Molecular basis of photochromism of a fluorescent protein revealed by direct ${ }^{13} \mathrm{C}$ detection under laser illumination. J Biomol NMR 48, 237-46 (2010).

144. Brakemann, T., Stiel, A. C., Weber, G., Andresen, M., Testa, I., Grotjohann, T., Leutenegger, M., Plessmann, U., Urlaub, H., Eggeling, C., Wahl, M. C., Hell, S. W. \& Jakobs, S. A reversibly photoswitchable GFP-like protein with fluorescence excitation decoupled from switching. Nat Biotechnol 29, 942-7 (2011).

145. Arai, Y., Takauchi, H., Ogami, Y., Fujiwara, S., Nakano, M., Matsuda, T. \& Nagai, T. Spontaneously Blinking Fluorescent Protein for Simple Single Laser Super-Resolution Live Cell Imaging. ACS Chem Biol 13, 1938-43 (2018).

146. Cranfill, P. J., Sell, B. R., Baird, M. A., Allen, J. R., Lavagnino, Z., de Gruiter, H. M., Kremers, G. J., Davidson, M. W., Ustione, A. \& Piston, D. W. Quantitative assessment of fluorescent proteins. Nat Methods 13, 557-62 (2016).

147. Heppert, J. K., Dickinson, D. J., Pani, A. M., Higgins, C. D., Steward, A., Ahringer, J., Kuhn, J. R. \& Goldstein, B. Comparative assessment of fluorescent proteins for in vivo imaging in an animal model system. Mol Biol Cell 27, 3385-94 (2016).

148. Nienhaus, K. \& Nienhaus, G. U. Photoswitchable Fluorescent Proteins: Do Not Always Look on the Bright Side. ACS Nano, 9104-8 (2016).

149. Hell, S. W. Microscopy and its focal switch. Nat Methods 6, 24-32 (2009).

150. Hell, S. W., Jakobs, S. \& Kastrup, L. Imaging and writing at the nanoscale with focused visible light through saturable optical transitions. Applied Physics A 77, 859-60 (2003). 
151. Testa, I., Urban, N. T., Jakobs, S., Eggeling, C., Willig, K. I. \& Hell, S. W. Nanoscopy of living brain slices with low light levels. Neuron 75, 992-1000 (2012).

152. Ratz, M., Testa, I., Hell, S. W. \& Jakobs, S. CRISPR/Cas9-mediated endogenous protein tagging for RESOLFT super-resolution microscopy of living human cells. Sci Rep 5, 9592 (2015).

153. Schnorrenberg, S., Grotjohann, T., Vorbrüggen, G., Herzig, A., Hell, S. W. \& Jakobs, S. In vivo super-resolution RESOLFT microscopy of Drosophila melanogaster. eLife $\mathbf{5}$, e15567 (2016).

154. Lavoie-Cardinal, F., Jensen, N. A., Westphal, V., Stiel, A. C., Chmyrov, A., Bierwagen, J., Testa, I., Jakobs, S. \& Hell, S. W. Two-color RESOLFT nanoscopy with green and red fluorescent photochromic proteins. Chemphyschem 15, 655-63 (2014).

155. Dreier, J., Castello, M., Coceano, G., Caceres, R., Plastino, J., Vicidomini, G. \& Testa, I. Smart scanning for low-illumination and fast RESOLFT nanoscopy in vivo. Nat Commun 10, 556 (2019).

156. Chmyrov, A., Keller, J., Grotjohann, T., Ratz, M., d'Este, E., Jakobs, S., Eggeling, C. \& Hell, S. W. Nanoscopy with more than 100,000 'doughnuts'. Nat Methods 10, 737-40 (2013).

157. Chmyrov, A., Leutenegger, M., Grotjohann, T., Schönle, A., Keller-Findeisen, J., Kastrup, L., Jakobs, S., Donnert, G., Sahl, S. J. \& Hell, S. W. Achromatic light patterning and improved image reconstruction for parallelized RESOLFT nanoscopy. Sci Rep 7, 44619 (2017).

158. Wang, S., Chen, X., Chang, L., Xue, R., Duan, H. \& Sun, Y. GMars-Q Enables Long-Term Live-Cell Parallelized Reversible Saturable Optical Fluorescence Transitions Nanoscopy. ACS Nano 10, 9136-44 (2016).

159. Masullo, L. A., Bodén, A., Pennacchietti, F., Coceano, G., Ratz, M. \& Testa, I. Enhanced photon collection enables four dimensional fluorescence nanoscopy of living systems. Nat Commun 9, 3281 (2018).

160. Li, D., Shao, L., Chen, B. C., Zhang, X., Zhang, M., Moses, B., Milkie, D. E., Beach, J. R., Hammer J. A., 3., Pasham, M., Kirchhausen, T., Baird, M. A., Davidson, M. W., $\mathrm{Xu}$, P. \& Betzig, E. ADVANCED IMAGING. Extended-resolution structured illumination imaging of endocytic and cytoskeletal dynamics. Science 349, aab3500 (2015).

161. Lu-Walther, H. W., Hou, W., Kielhorn, M., Arai, Y., Nagai, T., Kessels, M. M., Qualmann, B. \& Heintzmann, R. Nonlinear Structured Illumination Using a Fluorescent Protein Activating at the Readout Wavelength. PLoS One 11, e0165148 (2016). 
162. Quérard, J., Zhang, R., Kelemen, Z., Plamont, M. A., Xie, X., Chouket, R., Roemgens, I., Korepina, Y., Albright, S., Ipendey, E., Volovitch, M., Sladitschek, H. L., Neveu, P., Gissot, L., Gautier, A., Faure, J. D., Croquette, V., Le Saux, T. \& Jullien, L. Resonant out-of-phase fluorescence microscopy and remote imaging overcome spectral limitations. Nat Commun 8, 969 (2017).

163. Zhao, H., Ma, J., Ingaramo, M., Andrade, E., MacDonald, J., Ramsay, G., Piszczek, G., Patterson, G. H. \& Schuck, P. Accounting for photophysical processes and specific signal intensity changes in fluorescence-detected sedimentation velocity. Anal Chem 86, 9286-92 (2014).

164. Zhao, H., Fu, Y., Glasser, C., Andrade Alba, E. J., Mayer, M. L., Patterson, G. \& Schuck, P. Monochromatic multicomponent fluorescence sedimentation velocity for the study of high-affinity protein interactions. eLife 5, e17812 (2016).

165. Tuijtel, M. W., Koster, A. J., Jakobs, S., Faas, F. G. A. \& Sharp, T. H. Correlative cryo super-resolution light and electron microscopy on mammalian cells using fluorescent proteins. Sci Rep 9, 1369 (2019).

166. Schindelin, J., Arganda-Carreras, I., Frise, E., Kaynig, V., Longair, M., Pietzsch, T., Preibisch, S., Rueden, C., Saalfeld, S., Schmid, B., Tinevez, J.-Y., White, D. J., Hartenstein, V., Eliceiri, K., Tomancak, P. \& Cardona, A. Fiji: an open-source platform for biological-image analysis. Nat Methods 9, 676-682 (2012).

167. Schneider, C. A., Rasband, W. S. \& Eliceiri, K. W. NIH Image to ImageJ: 25 years of image analysis. Nat Methods 9, 671-675 (2012).

168. Shaner, N. C., Lambert, G. G., Chammas, A., Ni, Y., Cranfill, P. J., Baird, M. A., Sell, B. R., Allen, J. R., Day, R. N., Israelsson, M., Davidson, M. W. \& Wang, J. A bright monomeric green fluorescent protein derived from Branchiostoma lanceolatum. Nat Methods 10, 407-9 (2013).

169. Fuchs, J., Böhme, S., Oswald, F., Hedde, P. N., Krause, M., Wiedenmann, J. \& Nienhaus, G. U. A photoactivatable marker protein for pulse-chase imaging with superresolution. Nat Methods 7, 627-30 (2010).

170. Hein, B., Willig, K. I. \& Hell, S. W. Stimulated emission depletion (STED) nanoscopy of a fluorescent protein-labeled organelle inside a living cell. PNAS 105, 14271-6 (2008).

171. Zacharias, D. A., Violin, J. D., Newton, A. C. \& Tsien, R. Y. Partitioning of LipidModified Monomeric GFPs into Membrane Microdomains of Live Cells. Science 296, 913-6 (2002).

172. Wall, M. A., Socolich, M. \& Ranganathan, R. The structural basis for red fluorescence in the tetrameric GFP homolog DsRed. Nat Struct Biol 7, 1133-8 (2000). 
173. Stevens, F. J. Analysis of protein-protein interaction by simulation of small-zone size exclusion chromatography. Stochastic formulation of kinetic rate contributions to observed high-performance liquid chromatography elution characteristics. Biophys $J \mathbf{5 5}$, 1155-67 (1989).

174. Danzl, J. G., Sidenstein, S. C., Gregor, C., Urban, N. T., Ilgen, P., Jakobs, S. \& Hell, S. W. Coordinate-targeted fluorescence nanoscopy with multiple off states. Nature Photonics 10, 122-128 (2016).

175. Kamper, M., Ta, H., Jensen, N. A., Hell, S. W. \& Jakobs, S. Near-infrared STED nanoscopy with an engineered bacterial phytochrome. Nat Commun 9, 4762 (2018).

176. Duan, C., Adam, V., Byrdin, M., Ridard, J., Kieffer-Jaquinod, S., Morlot, C., Arcizet, D., Demachy, I. \& Bourgeois, D. Structural evidence for a two-regime photobleaching mechanism in a reversibly switchable fluorescent protein. J Am Chem Soc 135, 1584150 (2013).

177. Wäldchen, S., Lehmann, J., Klein, T., van de Linde, S. \& Sauer, M. Light-induced cell damage in live-cell super-resolution microscopy. Sci Rep 5, 15348 (2015).

178. Li, X., Chung, L. W., Mizuno, H., Miyawaki, A. \& Morokuma, K. Primary Events of Photodynamics in Reversible Photoswitching Fluorescent Protein Dronpa. The Journal of Physical Chemistry Letters 1, 3328-33 (2010).

179. Warren, M. M., Kaucikas, M., Fitzpatrick, A., Champion, P., Sage, J. T. \& van Thor, J. J. Ground-state proton transfer in the photoswitching reactions of the fluorescent protein Dronpa. Nat Commun 4, 1461 (2013).

180. Berardozzi, R., Adam, V., Martins, A. \& Bourgeois, D. Arginine 66 Controls DarkState Formation in Green-to-Red Photoconvertible Fluorescent Proteins. J Am Chem Soc 138, 558-65 (2016).

181. Adam, V., Nienhaus, K., Bourgeois, D. \& Nienhaus, G. U. Structural basis of enhanced photoconversion yield in green fluorescent protein-like protein Dendra2. Biochemistry 48, 4905-15 (2009).

182. Moeyaert, B., Nguyen Bich, N., De Zitter, E., Rocha, S., Clays, K., Mizuno, H., van Meervelt, L., Hofkens, J. \& Dedecker, P. Green-to-red photoconvertible Dronpa mutant for multimodal super-resolution fluorescence microscopy. ACS Nano 8, 1664-73 (2014).

183. Nienhaus, K., Nienhaus, G. U., Wiedenmann, J. \& Nar, H. Structural basis for photoinduced protein cleavage and green-to-red conversion of fluorescent protein EosFP. PNAS 102, 9156-9 (2005).

184. Tsutsui, H., Karasawa, S., Shimizu, H., Nukina, N. \& Miyawaki, A. Semi-rational engineering of a coral fluorescent protein into an efficient highlighter. EMBO Rep $\mathbf{6}$, 233-8 (2005). 
185. Tubbs, J. L., Tainer, J. A. \& Getzoff, E. D. Crystallographic structures of Discosoma red fluorescent protein with immature and mature chromophores: linking peptide bond trans-cis isomerization and acylimine formation in chromophore maturation. Biochemistry 44, 9833-40 (2005).

186. Fron, E., Van der Auweraer, M., Moeyaert, B., Michiels, J., Mizuno, H., Hofkens, J. \& Adam, V. Revealing the excited-state dynamics of the fluorescent protein Dendra2. $J$ Phys Chem B 117, 2300-13 (2013).

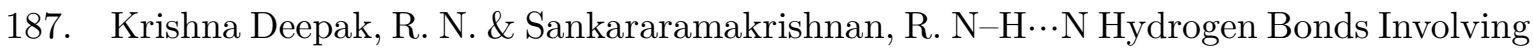
Histidine Imidazole Nitrogen Atoms: A New Structural Role for Histidine Residues in Proteins. Biochemistry 55, 3774-83 (2016).

188. Habuchi, S., Cotlet, M., Gensch, T., Bednarz, T., Haber-Pohlmeier, S., Rozenski, J., Dirix, G., Michiels, J., Vanderleyden, J., Heberle, J., De Schryver, F. C. \& Hofkens, J. Evidence for the isomerization and decarboxylation in the photoconversion of the red fluorescent protein DsRed. J Am Chem Soc 127, 8977-84 (2005).

189. Shaner, N. C., Lin, M. Z., McKeown, M. R., Steinbach, P. A., Hazelwood, K. L., Davidson, M. W. \& Tsien, R. Y. Improving the photostability of bright monomeric orange and red fluorescent proteins. Nat Methods 5, 545-51 (2008).

190. Roy, A., Field, M. J., Adam, V. \& Bourgeois, D. The nature of transient dark states in a photoactivatable fluorescent protein. J Am Chem Soc 133, 18586-9 (2011).

191. Adam, V., Carpentier, P., Violot, S., Lelimousin, M., Darnault, C., Nienhaus, G. U. \& Bourgeois, D. Structural basis of X-ray-induced transient photobleaching in a photoactivatable green fluorescent protein. J Am Chem Soc 131, 18063-5 (2009).

192. Don Paul, C., Traore, D. A., Olsen, S., Devenish, R. J., Close, D. W., Bell, T. D., Bradbury, A., Wilce, M. C. \& Prescott, M. X-Ray Crystal Structure and Properties of Phanta, a Weakly Fluorescent Photochromic GFP-Like Protein. PLoS One 10, e0123338 (2015).

193. Mizuno, H., Dedecker, P., Ando, R., Fukano, T., Hofkens, J. \& Miyawaki, A. Higher resolution in localization microscopy by slower switching of a photochromic protein. Photochem Photobiol Sci 9, 239-48 (2010).

194. Nguyen Bich, N., Moeyaert, B., Van Hecke, K., Dedecker, P., Mizuno, H., Hofkens, J. \& Van Meervelt, L. Structural basis for the influence of a single mutation K145N on the oligomerization and photoswitching rate of Dronpa. Acta Crystallogr D Biol Crystallogr 68, 1653-9 (2012).

195. Willig, K. I., Stiel, A. C., Brakemann, T., Jakobs, S. \& Hell, S. W. Dual-label STED nanoscopy of living cells using photochromism. Nano Lett 11, 3970-3 (2011).

196. Slade, K. M., Baker, R., Chua, M., Thompson, N. L. \& Pielak, G. J. Effects of recombinant protein expression on green fluorescent protein diffusion in Escherichia coli. Biochemistry 48, 5083-9 (2009). 
197. Ito, T., Oshita, S., Nakabayashi, T., Sun, F., Kinjo, M. \& Ohta, N. Fluorescence lifetime images of green fluorescent protein in HeLa cells during TNF- $\alpha$ induced apoptosis. Photochem Photobiol Sci 8, 763-7 (2009).

198. Berezin, M. Y. \& Achilefu, S. Fluorescence Lifetime Measurements and Biological Imaging. Chem Rev 110, 2641-84 (2010).

199. Stelzer, E. H. K. Light-sheet fluorescence microscopy for quantitative biology. Nat Methods 12, 23-6 (2015).

200. Böhm, U., Hell, S. W. \& Schmidt, R. 4Pi-RESOLFT nanoscopy. Nat Commun 7, 10504 (2016).

201. Hui-wang, A., Shaner, N. C., Cheng, Z., Tsien, R. Y. \& Campbell, R. E. Exploration of New Chromophore Structures Leads to the Identification of Improved Blue Fluorescent Proteins. Biochemistry 46, 5904-10 (2007).

202. Jakobs, S., Subramaniam, V., Schönle, A., Jovin, T. M. \& Hell, S. W. EFGP and DsRed expressing cultures of Escherichia coli imaged by confocal, two-photon and fluorescence lifetime microscopy. FEBS Lett 479, 131-5 (2000).

203. Dower, J. D., Miller, J. F. \& Ragsdale, C. W. High efficiency transformation of E. coli by high voltage electroporation. Nucleic Acids Res 16, 6127-45 (1988).

204. Riedl, J., Crevenna, A. H., Kessenbrock, K., Yu, J. H., Neukirchen, D., Bista, M., Bradke, F., Jenne, D., Holak, T. A., Werb, Z., Sixt, M. \& Wedlich-Soldner, R. Lifeact: a versatile marker to visualize F-actin. Nat Methods 5, 605-7 (2008).

205. Lamesch, P., Li, N., Milstein, S., Fan, C., Hao, T., Szabo, G., Hu, Z., Venkatesan, K., Bethel, G., Martin, P., Rogers, J., Lawlor, S., McLaren, S., Dricot, A., Borick, H., Cusick, M. E., Vandenhaute, J., Dunham, I., Hill, D. E. \& Vidal, M. hORFeome v3.1: a resource of human open reading frames representing over 10,000 human genes. Genomics 89, 307-15 (2007).

206. Morozova, K. S., Piatkevich, K. D., Gould, T. J., Zhang, J., Bewersdorf, J. \& Verkhusha, V. V. Far-red fluorescent protein excitable with red lasers for flow cytometry and superresolution STED nanoscopy. Biophys J 99, L13-5 (2010).

207. Rizzuto, R., Nakase, H., Darras, B., Francke, U., Fabrizi, G. M., Mengel, T., Walsh, F., Kadenbach, B., DiMauro, S. \& Schon, E. A. A gene specifying subunit VIII of human cytochrome c oxidase is localized to chromosome 11 and is expressed in both muscle and non-muscle tissues. J Biol Chem 264, 10595-600 (1989).

208. Rizzuto, R., Brini, M., Pizzo, P., Murgia, M. \& Pozzan, T. Chimeric green fluorescent protein as a tool for visualizing subcellular organelles in living cells. Curr Biol 5, 63542 (1995).

209. Sawano, A. \& Miyawaki, A. Directed evolution of green fluorescent protein by a new versatile PCR strategy for site-directed and semi-random mutagenesis. Nucleic Acids Res 28, e78 (2000). 
210. Bradford, M. M. A rapid and sensitive method for the quantitation of microgram quantities of protein utilizing the principle of protein-dye binding. Anal Biochem 72, 248-54 (1976).

211. E., G., C., H., A., G., S., D., R., W. M., D., A. R. \& A., B. in The Proteomics Protocols Handbook (ed Walker, J. M.) 571-607 (Humana Press, 2005).

212. Linkert, M., Rueden, C. T., Allan, C., Burel, J. M., Moore, W., Patterson, A., Loranger, B., Moore, J., Neves, C., Macdonald, D., Tarkowska, A., Sticco, C., Hill, E., Rossner, M., Eliceiri, K. W. \& Swedlow, J. R. Metadata matters: access to image data in the real world. J Cell Biol 189, 777-82 (2010). 


\section{Acronyms}

(f)PALM (fluorescence) photoactivated localization microscopy

$\mathrm{AF}$

APD

CenpC1

CW

DMEM

DNA

dSTORM

EDTA

$\mathrm{EP}$

ER

ESPT

FACS

FAD

FBS

FLIM

FP

FPGA

FRAP

FWHM

GSD

GSDIM

LB

Map2

MCS

MSM

$\mathrm{NADH}$

NA

NIR

Nup50

PA NL SIM

PAFP

PAGE

PAINT

PALMIRA

PBS

PCFP

PCR

\author{
autofocus
}

avalanche photodiode

centromere protein $\mathrm{C} 1$

continuous wave

Dulbecco's Modified Eagle Medium

desoxyribonucleic acid

$$
\text { direct STORM }
$$

ethylenediaminetetraacetate

error-prone

endoplasmic reticulum

excited state proton transfer

fluorescence activated cell sorting

flavine adenine dinucleotide

fetal bovine serum

fluorescence lifetime imaging

fluorescent protein

field programmable gate array

fluorescence recovery after photobleaching

full width half maximum

ground-state depletion

ground-state depletion microscopy followed by individual molecule return

lysogeny broth

microtubule-associated protein 2

multiple cloning site

multiple site mutagenesis

nicotinamide adenine dinucleotide

numerical aperture

near infrared

nucleoporin $50 \mathrm{kDa}$

patterned activation non-linear SIM

photoactivatable fluorescent protein

polyacrylamide gel electrophoresis

points accumulation for imaging in nanoscale topography

PALM with independently running acquisition

phosphate buffered saline

photoconvertible fluorescent protein

polymerase chain reaction 
PMT photomultiplier tube

$\mathrm{PSF}$

point spread function

PTS

peroxisomal targeting sequence

RESOLFT reversible saturable optical linear fluorescence transition

RSFP

SDM

reversibly switchable fluorescent protein

SDS

site-directed mutagenesis

SEC

sodium dodecyl sulfate

SIM

size exclusion chromatography

STED

structured illumination microscopy

stimulated emission depletion

STORM

stochastic optical reconstruction microscopy

TAE

Tris acetat EDTA

TEMED

N,N,N',N'-Tetramethylethylendiamin

UV

ultraviolett

VIS

visible

VPP

vortex phase plate 


\section{Acknowledgements}

An dieser Stelle möchte ich allen Beteiligten danken, die, auf die ein oder andere Art und Weise, direkt oder indirekt, an der Entstehung dieser Doktorarbeit durch ihre Hilfe und ihren Beistand beteiligt waren.

Mein großer Dank geht zuallererst an Prof. Dr. Stefan Jakobs für die Betreuung dieses Projekts und die Möglichkeit, daran zu arbeiten, sowie an Prof. Dr. Stefan W. Hell für das hervorragende wissenschaftliche Umfeld im Institut und die Möglichkeit, daran teilzuhaben. Weiterhin danke ich Prof. Dr. Kai Tittmann für die Begutachtung dieser Arbeit und seine Anmerkungen und Ratschläge während der Betreuungsausschusstreffen. Ebenso geht mein Dank an apl. Prof. Dr. Alexander Egner, Prof. Dr. Stefan W. Hell, PD Dr. Wilfried Kramer und PD Dr. Thomas Teichmann für ihre Bereitschaft, diese Arbeit als Mitglieder der Prüfungskommission zu bewerten.

Ein riesiges Dankeschön geht an alle Mitglieder der Abteilung Nanobiophotonics, von denen ich in den letzten Jahren so viel gelernt habe!

An Dr. Jan Keller-Findeisen für die Hilfe mit den vielen Einsen und Nullen, an Dr. Marcel Leutenegger, Jasmin Pape, Michael Weber und Philipp Alt für das geduldige Erläutern von physikalischen und technischen Hintergründen und für die Hilfe bei der Suche nach dem Wurm im System.

An Dr. Martin Andresen, Dr. Tim Grotjohann und Dr. Sebastian Schnorrenberg für die Überlassung ihrer mit Liebe geschraubten Geräte. Und natürlich dafür, mich mit selbigen bekannt gemacht zu haben.

An Sylvia Löbermann, Tanja Gilat, Ellen Rothermel und Rita Schmitz-Salue für die phänomenale und tatkräftige Unterstüzung, Toleranz gegenüber chaotischen Laborbänken, Schokolade und jedes liebe Lächeln auf dem Flur. Und dafür, immer das richtige Eppi in der Hinterhand zu haben. Natürlich auch an Jay Jethwa, der immer die richtige Schraube zur Hand hatte, dem nie die Batterien ausgingen und der alles immer in Ordnung gebracht hat schon bevor es Probleme gab. Und der wahrscheinlich auch jede Anleitung und Dokumentation abgeheftet hat, bevor sie überhaupt geschrieben ist.

Herausragende und überschwängliche Dankbarkeit geht an Dr. Nickels Jensen. Für die aufopfernde Hilfe bei allen großen und kleinen Problemen, für sein buntes Wissen über die Proteine, für die Geduld und Aufmerksamkeit. Für unfassbar konstruktives Feedback und dafür, dass ich jetzt (fast) seine Schrift lesen kann. Und natürlich danke an Nickels als Laborpapa und den besten Gastgeber Göttingens für den ganzen Rest: Hervorragende Drinks, grandiose Snacks, übergreifende Begeisterungsfähigkeit, für jeden dummen Spruch, für gute, schlechte und fürchterliche Filme. 
An Nickels, Daniel, Jan und Sebastian fürs Korrekturlesen und das Aufspüren von von Dopplungen, Tippfhelern und Fettnäpfchen.

An Peter, Carola, Till, Joana und Isy für das Ertragen der erhöhten Klapperfrequenz meiner Tastatur und der Müllkippe, die mein Schreibtisch momentan ist. Ganz besonders an meine Arbeitsehefrau Isy für die langjährige Treue, Schokolade, Burgerfahrten und ihre Organisiertheit.

Und dankedankedanke an alle, die Göttingen zu einem guten Ort gemacht haben, auch abseits der Wissenschaft: Maria und Andi für die besten, festesten Umarmungen, Sebastian für seinen Zuspruch, wenn das Protein mal nicht so wollte wie es sollte, Elke für den besten Irrsinn, Steffi und Philipp für das herausragende Freizeitmanagement, Dirk für ein Stück Emsland, Christian dafür, dass er meinen literarischen Horizont erweitert hat, Daniel für vielleicht eine der letzten WG Parties, Florian für abartig guten Banoffi-Pie, Dirk, Peter, Tanja, Stefan, Jennifer, Daniel, Sarah, Axel, Felix, Till und alle, die ich noch vergessen habe, für Dinge, die mir gerade nicht einfallen, die aber eigentlich unbedingt gewürdigt werden müssten. Man möge es mir verzeihen.

Vielen, vielen Dank auch an alle Freunde zuhause und überall anders, die in letzer Zeit zu kurz gekommen sind und mir das hoffentlich nicht krumm nehmen. Im nächsten Jahr wird alles nachgeholt!

Der gleiche Dank geht auch an meine Eltern, Wencke, Carsten, Lasse und Fiete und alle Diekmänner. Jetzt habe ich wieder mehr Zeit für euch! Danke für eure fortwährende Unterstützung!

Und an Hanna. Ganz besonders an Hanna, die im letzten Jahr immer für mich da war und das alles mitertragen hat. Ohne dich wäre das ganz anders gelaufen. 